\title{
On the Thermodynamics of Gravity Based on the Equivalence of Energy and Mass and Spacetime
}

\author{
Akira Kawamura*
}

\begin{abstract}
Starting from the assumption of equivalence of energy and mass and spacetime, we explain macroscopic and microscopic gravitational phenomena in terms of thermodynamics. Spacetime is quantized by particles with the minimum mass. We call that particle the spacetime particle. Gravity is represented as a thermodynamic pressure caused by spacetime particles. Then we derived the equation of state of spacetime particles. That equation and so on lead us to conclude the following. Dark matter is spacetime particles composing spacetime. The basic mass of the spacetime particle is $1.05 \times 10^{-62} \mathrm{~kg}$. Spacetime structure is represented as the quantized 3-dimensional sphere of Poincaré conjecture. An elementary particle is composed of spacetime particles with energy and charged particles with charges, and it has a radius whose rotational speed is the speed of light. Dark energy is a virtual negative mass by the mass reduction of ordinary matter. Before the birth of the universe, spacetime was the imaginary spacetime caused by one black hole, and spacetime was repeatedly created and annihilated. The universe was born by the collapse of that black hole. Inflation was caused by the expansion of individual matter to their radius at superluminal speed.
\end{abstract}

\section{Introduction}

Physics on gravity and energy has many problems that need to be solved. For example, relativity theory has the following problems. It can't explain dark matter, which is an unknown source of gravity. It can't explain dark energy, which accelerates the expansion of spacetime. It can't explain inflation, which is the expansion of spacetime at superluminal speed just after the birth of the universe. It has a singularity within black holes and at

*Electronic address: anytotalresearch@yahoo.co.jp 
the birth of the universe. It is hard to unify relativity and quantum theory, and it is hard to quantize gravity. Quantum theory has the following problems. It can't explain a wave function well, which is expressed by a complex number. It can't explain the shape of elementary particles without contradiction.

Conventional research has attempted to solve these problems by considering them as an extension of existing physics or by modifying existing physics. However, conventional research cannot explain macroscopic gravitational phenomena even if it can quantize gravity, or it cannot quantize gravity even if it can explain macroscopic gravitational phenomena. In this way, it is hard to solve both macroscopic and microscopic problems with conventional research methods.

The purpose of this paper is to propose a new gravitational theory that is different from existing physics and to solve both macroscopic and microscopic problems associated with gravity. As guiding principles for this purpose, we assume the following six principles.

- Pair principle: almost every element has an inverse or pair element, which is inverse or different but can be considered as one combination.

- Complementarity principle: a complete description can be made by considering that mutually contradictory elements are realized simultaneously or are the same.

- Quantum principle: every element can be quantized by the smallest element and can be described based on particles.

- Relativity principle: if a phenomenon belongs to the same frame of reference as an observer, the physical law of the phenomenon can be described as the same form in that frame of reference.

- Simplicity principle: it is preferable to be simpler and easier theories and equations, which are naturally derived and can be concretely imagined and have unified regularity.

- Parsimony principle: it is preferable to be able to explain more things from fewer equations or assumptions.

The pair principle and the complementarity principle are used to come up with valid ideas for the new theory. The quantum principle and the relativity principle are used to create a theory based on the ideas of quantum and relativity theory. The simplicity principle and the parsimony principle are used as criteria for judging the validity of the new theory. The six principles lead us to conclude the following. Energy and mass and spacetime are equivalent. Spacetime is quantized by spacetime particles with the minimum mass. Gravity is represented as a thermodynamic pressure caused by spacetime particles. In this paper, we propose the new gravitational theory based on six principles and explain macroscopic and microscopic gravitational phenomena in terms of thermodynamics. 
Note that the gravitational theory in this paper does not necessarily deny existing physics. The complementarity principle indicates that a phenomenon can be described from not only one viewpoint but also another viewpoint. For example, gravity can be described as not only Newton's law of universal gravity but also a thermodynamic pressure caused by spacetime particles. Therefore, the gravitational theory in this paper only describes gravity from a different viewpoint from existing physics according to the complementarity principle.

\section{Derivation of the Gravity Equation of Thermodynamic Gravity Form}

In this section, from some assumptions, we derive the gravity equation of thermodynamic gravity form and the equation of state of spacetime particles. From these equations and so on, we define mass and spacetime, and we explain an inertial force.

\subsection{Derivation of the Gravity Equation of Thermodynamic Gravity Form}

From some assumptions, we derive the gravity equation of thermodynamic gravity form.

Assumption 2.1: Energy and mass and spacetime are equivalent. First, the uncertainty principle for spacetime suggests that spacetime itself has energy or mass. Second, the pair principle suggests that the virtual negative mass, which consumes mass or energy, expands spacetime. Since the positive mass contracts spacetime, the negative mass expands spacetime from the pair principle. However, the existence of negative mass has not been confirmed, so the virtual negative mass, which consumes mass or energy, expands spacetime. Third, if the virtual negative mass expands spacetime, judging from reversibility, we can in principle extract mass or energy from spacetime. From these, we conclude assumption 2.1. This means that mass or energy can be converted into spacetime, and spacetime can be converted into mass or energy.

Assumption 2.2: Spacetime is quantized by spacetime particles with the minimum mass $m_{s p}$, and spacetime is a group of spacetime particles. The spacetime particle is the name of the particle composing spacetime, and $m_{s p}$ is the mass of one spacetime particle. First, the equivalence of assumption 2.1 suggests that spacetime has mass or energy. We can regard things with energy or mass as particles. Therefore, spacetime is quantized by spacetime particles, and spacetime is composed of spacetime particles. Second, spacetime particles have the smallest mass, and that mass is smaller than the mass of any other particle. This is because one spacetime particle represents the minimum spacetime, and the minimum spacetime is equivalent to the minimum mass from the equivalence of assumption 2.1. From these, we conclude assumption 2.2. 
Assumption 2.3: Spacetime particles obey the ideal gas law

$$
P=\frac{\mathrm{d} N}{\mathrm{~d} V} \mathrm{k}_{\mathrm{B}} T
$$

where $P, V, N$ and $T$ are the pressure, volume, number and temperature of spacetime particles, and $\mathrm{k}_{\mathrm{B}}$ is the Boltzmann constant.

First, spacetime particles have a temperature. Since spacetime particles have mass, they have kinetic energy. The temperature of particles is related to the kinetic energy of the particles. That is, if particles have kinetic energy, they have a temperature. Therefore, spacetime particles have a temperature, like ordinary particles. This can also be explained by the Unruh effect $[1,2,3]$. From the Unruh effect, an accelerating observer in a vacuum should observe a thermal bath. A vacuum is spacetime without ordinary matter, and spacetime is a group of spacetime particles. That is, the Unruh temperature is the temperature of spacetime particles. Therefore, we conclude that spacetime particles have a temperature.

Second, spacetime particles are gravitons carrying gravity. From the general theory of relativity, gravity is the curvature of spacetime. This suggests that the graviton is spacetime itself. From assumption 2.2, spacetime is a group of spacetime particles. Therefore, we conclude that spacetime particles are gravitons carrying gravity.

Third, gravity is the pressure according to the density and temperature of spacetime particles. The spacetime particle number density is proportional to gravity because it is related to the strength of gravity. Spacetime particles have a temperature. Pressure is well known as the force according to density and temperature. Black hole thermodynamics [4, 5] and entropic gravity [6] suggest that gravity is related to thermodynamics. Therefore, we conclude that gravity is the pressure according to the density and temperature of spacetime particles.

Fourth, the spacetime particle number density is expressed in the differential form of $\frac{\mathrm{d} N}{\mathrm{~d} V}$. Since the spacetime particle number density is proportional to gravity, it changes with space. Therefore, the spacetime particle number density is expressed in the differential form of $\frac{\mathrm{d} N}{\mathrm{~d} V}$ as a function of space $V$.

Fifth, the ideal gas law is the most general equation of state including three elements: pressure, temperature, and density.

From these, we conclude assumption 2.3.

Assumption 2.4: The relationship between the gravity $F$ and the pressure $P$ of spacetime particles is expressed as

$$
F=\int_{S} \vec{P} \cdot \mathrm{d} \vec{S}=S \times P,
$$

where $\vec{P}$ is the pressure vector of spacetime particles, $\mathrm{d} \vec{S}$ is an infinitesimal area vector, and $S$ is the area of the closed surface and is the area expressed as 


$$
S=4 \pi r^{2}
$$

where $r$ is the radius from mass. First, since gravity is the pressure multiplied by area, gravity $F$ is expressed as $F=S \times P$. Second, the temperature $T$ and the spacetime particle number density $\frac{\mathrm{d} N}{\mathrm{~d} V}$ are proportional to gravity $F$. This is because they are related to the strength of gravity. Third, since the temperature $T$ is proportional to gravity $F$, from equation (2.1) and $F=S \times P$, the spacetime particle number density $\frac{\mathrm{d} N}{\mathrm{~d} V}$ is inversely proportional to the area $S$. Fourth, from Newton's law of universal gravitation, gravity $F$ is inversely proportional to the square of radius $r$. Fifth, from these relationships, the area $S$ is proportional to the square of radius $r$, and gravity $F$ is inversely proportional to the area $S$. Sixth, since gravity $F$ is inversely proportional to the area $S$, the area $S$ is constant over the entire surface of the sphere where gravity $F$ is constant. Seventh, from analogy with electric fields, Gauss's law should apply to gravitational fields as well. Therefore, the area $S$ is the area of the closed surface. From these, we conclude that the area $S$ is the surface area of the sphere where gravity $F$ is constant, and gravity $F$ is expressed as equation (2.2).

Assumption 2.5: The temperature $T$ of spacetime particles is expressed as

$$
T=\frac{m}{M} \times T_{s p} \times T_{u},
$$

where $m$ is the passive gravitational mass of an object that gravity is exerted on, $M$ is the active gravitational mass of an object that exerts gravity, $T_{s p}$ is the dimensionless temperature of spacetime particles, and $T_{u}$ is the Unruh temperature expressed as [7]

$$
T_{u}=\frac{\mathrm{k}_{\mathrm{sp}}}{\mathrm{k}_{\mathrm{B}}} \times a,
$$

where $a$ is acceleration received by mass $m$, and $\mathrm{k}_{\mathrm{sp}}$ is the constant expressed as

$$
\mathrm{k}_{\mathrm{sp}}=\frac{\hbar}{2 \pi \mathrm{c}},
$$

where $\mathrm{h}$ is Dirac's constant, and c is the speed of light.

First, the Unruh temperature $T_{u}$ is the temperature of a vacuum observed by the accelerating object. The temperature of a vacuum is the temperature of spacetime particles. Therefore, the temperature $T$ of spacetime particles is basically expressed by the Unruh temperature. Note that the acceleration $a$ in the Unruh temperature has no absolute value.

Second, the element $\frac{m}{M}$ is multiplied to make $\frac{\mathrm{d} N}{\mathrm{~d} V}$ the function of mass $M$ and $T$ the function of mass $m$. The Unruh temperature $T_{u}$ is the temperature of a vacuum observed by the accelerating object, so the acceleration $a$ is the gravitational acceleration by the mass $M$. Then, the density $\frac{\mathrm{d} N}{\mathrm{~d} V}$ becomes the function of mass $m$, and the temperature $T$ becomes the function of mass $M$. Spacetime particles are gravitons carrying gravity, so the 
spacetime particle number density $\frac{\mathrm{d} N}{\mathrm{~d} V}$ represents a gravitational field. It is unnatural that the gravitational field of mass $M$ is not represented by the function of mass $M$. Therefore, by multiplying the Unruh temperature by $\frac{m}{M}$, we make $\frac{\mathrm{d} N}{\mathrm{~d} V}$ the function of mass $M$ and $T$ the function of mass $m$.

Third, the temperature $T_{s p}$ is multiplied because it will be needed in later calculations. There are two reasons. The equation of state of spacetime particles is derived from the gravity equation of thermodynamic gravity form. Comparing the equation of state of spacetime particles with the ideal gas law, that equation requires a temperature parameter corresponding to the temperature $T$ of the ideal gas law. Moreover, that equation requires a dimensionless parameter to make the rotation speed of elementary particles the speed of light. Therefore, $T_{s p}$ is multiplied as the dimensionless temperature of spacetime particles. The reason why the temperature $T_{s p}$ becomes dimensionless is explained in section 4 .

From these, we conclude assumption 2.5.

We derive the gravity equation of thermodynamic gravity form. From Newton's law of universal gravitation, gravitational acceleration $a$ is expressed as

$$
a=-\frac{\mathrm{G} M}{r^{2}}
$$

where $\mathrm{G}$ is the universal gravitational constant. From equations (2.7) and (2.5), the Unruh temperature $T_{u}$ is expressed as

$$
T_{u}=-\frac{\mathrm{k}_{\mathrm{sp}}}{\mathrm{k}_{\mathrm{B}}} \times \frac{\mathrm{G} M}{r^{2}} .
$$

From equations (2.1) to (2.8), gravity $F$ is expressed as

$$
F=S \times \frac{\mathrm{d} N}{\mathrm{~d} V} \times \mathrm{k}_{\mathrm{B}} T,
$$

where $S$ is $4 \pi r^{2}, T$ is $\frac{m}{M} \times T_{s p} \times T_{u}, T_{u}$ is $-\frac{\mathrm{k}_{\mathrm{sp}}}{\mathrm{k}_{\mathrm{B}}} \times \frac{\mathrm{G} M}{r^{2}}$, and $\mathrm{k}_{\mathrm{sp}}$ is $\frac{\hbar}{2 \pi \mathrm{c}}$. Equation (2.9) is the gravity equation of thermodynamic gravity form. The gravity expressed by equation (2.9) is called thermodynamic gravity. In this way, the gravity equation of thermodynamic gravity form is derived.

We discuss the physical meaning of the temperature of spacetime particles. First, from a physical point of view, the thermodynamic temperature can't become negative. This is because a negative temperature means that particles have negative kinetic energy. Therefore, we conclude that the temperature itself of spacetime particles cannot be observed and only its force can be observed. Second, from a mathematical point of view, if the temperature is positive, the pressure is outward, if the temperature is negative, the pressure is inward, and if the temperature is zero, there is no pressure. Gravity works inward, so the temperature is negative. Third, from the first law of thermodynamics, we can explain the temperature as 
follows. If the temperature is negative, the pressure is inward, so when space expands, work is done from outside. This means that energy is supplied from outside. If the temperature is positive, the pressure is outward, so when space expands, work is done to outside. This means that internal energy is consumed. In summary, we conclude that the physical meaning of the temperature of spacetime particles is as follows. A positive temperature represents an outward pressure, a negative temperature represents an inward pressure, and zero temperature represents no pressure. In spacetime expansion, a negative temperature represents an external energy supply, and a positive temperature represents an internal energy consumption. The temperature itself of spacetime particles cannot be observed and only its force can be observed.

\subsection{Derivation of the Equation of State of Spacetime Particles}

From Newton's law of universal gravitation and the gravity equation of thermodynamic gravity form, we derive the equation of state of spacetime particles.

Assumption 2.6: Dark matter is spacetime particles composing spacetime. From assumption 2.2 , if spacetime has mass, the mass of the entire outer space will be enormous. Observations show that the proportion of ordinary matter in total energy is $4.9 \%$, the proportion of dark matter is $26.8 \%$, and the proportion of dark energy is $68.3 \%$ [8]. From this data, the mass of dark matter is more than five times larger than that of ordinary matter. Therefore, we conclude assumption 2.6.

Assumption 2.7: The distribution of spacetime particles is determined only by the mass of ordinary matter. From assumption 2.6, dark matter is spacetime particles composing spacetime. Observations show that the distribution of dark matter is proportional to the mass of ordinary matter. Therefore, we conclude assumption 2.7. Assumption 2.7 is that the distribution of spacetime particles is not affected by the mass of spacetime particles themselves.

Newton's law of universal gravitation is expressed as

$$
F=-\frac{\mathrm{G} M m}{r^{2}} .
$$

The gravity of equation (2.10) is called Newtonian gravity. From the complementarity principle, gravity can be expressed as not only Newtonian gravity but also thermodynamic gravity. As is obvious, no matter which way gravity is expressed, gravity must be the same. Therefore, from equations (2.9) and (2.10), gravity $F$ is expressed as

$$
F=-\frac{\mathrm{G} M m}{r^{2}}=S \times \frac{\mathrm{d} N}{\mathrm{~d} V} \times \mathrm{k}_{\mathrm{B}} T .
$$

From equations (2.4) and (2.8), the temperature $T$ of spacetime particles is expressed as 


$$
T=-\frac{m}{M} \times T_{s p} \times \frac{\mathrm{k}_{\mathrm{sp}}}{\mathrm{k}_{\mathrm{B}}} \times \frac{\mathrm{G} M}{r^{2}} .
$$

From equations (2.3) and (2.11) and (2.12), the spacetime particle number density $\frac{\mathrm{d} N}{\mathrm{~d} V}$ is expressed as

$$
\frac{\mathrm{d} N}{\mathrm{~d} V}=\frac{M}{4 \pi r^{2} \mathrm{k}_{\mathrm{sp}} T_{s p}} .
$$

The spacetime particle number density $\frac{\mathrm{d} N}{\mathrm{~d} V}$ for any area $S$ is expressed as

$$
\frac{\mathrm{d} N}{\mathrm{~d} V}=\frac{M}{S \mathrm{k}_{\mathrm{sp}} T_{s p}} .
$$

As $\mathrm{d} V=r^{2} \sin \theta \mathrm{d} \theta \mathrm{d} \phi \mathrm{d} r$, integrating equation (2.13) over the volume of a sphere with radius $r$, the spacetime particle number $N$ is derived as

$$
N=\frac{M r}{\mathrm{k}_{\mathrm{sp}} T_{s p}} .
$$

The ideal gas law is expressed as

$$
P V=N \mathrm{k}_{\mathrm{B}} T
$$

where $P, V, N$ and $T$ are the pressure, volume, number and temperature of the ideal gas. When we rearrange equation (2.15), it is expressed as

$$
M r=N \mathrm{k}_{\mathrm{sp}} T_{s p}
$$

Since equation (2.17) is the same form as the ideal gas law, it is the equation of state of spacetime particles. Comparing equation (2.16) with equation (2.17), $M, r, \mathrm{k}_{\mathrm{sp}}$, and $T_{s p}$ correspond to $P, V, \mathrm{k}_{\mathrm{B}}$, and $T$.

We mathematically define spacetime from the equation of state of spacetime particles.

First, spacetime is the spacetime particle number $N$. From assumption 2.2, spacetime is a group of spacetime particles. The spacetime particle number $N$ is a group of spacetime particles. Therefore, the spacetime particle number $N$ represents spacetime. For example, if the spacetime particle number $N$ is zero, it means that there is no spacetime.

Second, the spacetime radius is the radius $r$ where spacetime particles are distributed. From equation (2.15), the spacetime radius $r$ is expressed as

$$
r=N \times \frac{\mathrm{k}_{\mathrm{sp}} T_{s p}}{M} .
$$


From equation (2.18), if the spacetime particle number $N$ is zero, the spacetime radius $r$ is zero.

Third, spacetime density is expressed as the spacetime particle number density $\frac{\mathrm{d} N}{\mathrm{~d} V}$.

\subsection{Inertial Force and Definition of Mass}

From the derived equations and so on, we define mass and explain an inertial force.

First of all, we define mass.

- Passive gravitational mass: the mass of an object that gravity is exerted on, and the mass that is finally contained in the temperature $T$ of spacetime particles. For example, that mass is $m$ in $T=-T_{s p} \times \frac{\mathrm{k}_{\mathrm{sp}}}{\mathrm{k}_{\mathrm{B}}} \times \frac{\mathrm{G} m}{r^{2}}$.

- Active gravitational mass: the mass of an object that exerts gravity, and the mass that is contained in the spacetime particle number density $\frac{\mathrm{d} N}{\mathrm{~d} V}$. For example, that mass is $M$ in $\frac{\mathrm{d} N}{\mathrm{~d} V}=\frac{M}{4 \pi r^{2} \mathrm{k}_{\mathrm{sp}} T_{s p}}$.

- Inertial mass: the mass that represents the resistance when accelerating an object, and the mass that is included in equation $F=m a$.

In this way, in this paper, passive gravitational mass and active gravitational mass are clearly distinguished.

We discuss particles carrying inertial forces. First, an inertial force is a force received by mass. Second, the graviton is the particle that carries the force received by mass, and gravitons are spacetime particles. Third, the Unruh temperature is generated for any acceleration, and the Unruh temperature is the temperature of spacetime particles. Fourth, the temperature of spacetime particles causes pressure by spacetime particles. Therefore, we conclude that an inertial force is represented as the pressure caused by spacetime particles, and particles carrying inertial forces are spacetime particles.

We generalize the gravity equation of thermodynamic gravity form so that it can be applied to all forces caused by gravitons. Specifically, the gravity equation itself of thermodynamic gravity form has the same form, and only the individual parameters change. Acceleration $a$ is the acceleration exerted on the mass $m$, mass $m$ is the mass that the acceleration $a$ is exerted on, mass $M$ is the mass that exerts the acceleration $a$, and area $S$ is the area where the acceleration $a$ is constant. However, in the case of an inertial force, there is no mass $M$ that exerts acceleration $a$, so we assume the following.

Assumption 2.8: In the case of an inertial force, the mass $M$ that exerts the acceleration $a$ is equal to the mass $m$ that the acceleration $a$ is exerted on $(M=m)$.

From assumption 2.8 and so on, we discuss an inertial force. First, if active gravitational mass exists, the spacetime particle number density $\frac{\mathrm{d} N}{\mathrm{~d} V}$ is generated. Second, an inertial force 
is carried by gravitons, which are spacetime particles that compose the spacetime particle number density $\frac{\mathrm{d} N}{\mathrm{~d} V}$. Third, from $M=m$ of assumption 2.8, the spacetime particle number density $\frac{\mathrm{d} N}{\mathrm{~d} V}$ of an inertial force is generated by its own active gravitational mass. Therefore, we conclude that an inertial force works by the spacetime particle number density $\frac{\mathrm{d} N}{\mathrm{~d} V}$ by its own active gravitational mass.

From this, we conclude as follows. First, if the density $\frac{\mathrm{d} N}{\mathrm{~d} V}$ by its own active gravitational mass exists in the space where an object moves, an inertial force works in that space. If the density $\frac{\mathrm{d} N}{\mathrm{~d} V}$ by its own active gravitational mass does not exist in the space where an object moves, no inertial force works in that space, even if the object has active gravitational mass. Second, if an object has active gravitational mass and the density $\frac{\mathrm{d} N}{\mathrm{~d} V}$ by that mass exists in space, an inertial force works on the object and the object moves at less than the speed of light. If an object does not have active gravitational mass or the density $\frac{\mathrm{d} N}{\mathrm{~d} V}$ by that mass does not exist in space, an inertial force does not work on the object and the object moves at the speed of light.

We explain an inertial force in detail.

First, if an object has active gravitational mass, spacetime particles are distributed around the object, that is, the spacetime particle number density $\frac{\mathrm{d} N}{\mathrm{~d} V}$ is generated. If the object moves in uniform linear motion, the spacetime particle distribution will move at the same velocity as the object. When the object accelerates, the velocity of the object becomes faster than the velocity of the spacetime particle distribution. Therefore, the object at this time receives the resistance force from the spacetime particle distribution. Since the spacetime particle number density $\frac{\mathrm{d} N}{\mathrm{~d} V}$ is proportional to mass, the heavier mass is, the stronger the resistance force becomes. When the object decelerates, the velocity of the object becomes slower than the velocity of the spacetime particle distribution. Therefore, the object at this time receives the pushed force from the spacetime particle distribution. Since the spacetime particle number density $\frac{\mathrm{d} N}{\mathrm{~d} V}$ is proportional to the mass, the heavier the mass is, the stronger the pushed force becomes. In this way, an inertial force is the resistance force and pushed force from the spacetime particle distribution.

Second, if an object does not have active gravitational mass, spacetime particles are not distributed around the object, that is, the spacetime particle number density $\frac{\mathrm{d} N}{\mathrm{~d} V}$ is not generated. For this reason, even if the object moves in uniform linear motion, there is no spacetime particle distribution that moves at the same velocity as the object. Therefore, the object without active gravitational mass does not receive force from the spacetime particle distribution even when accelerated or decelerated. That is, an inertial force does not work on an object without active gravitational mass.

Third, from $M=m$ of assumption 2.8, in the case of an inertial force, the mass $M$ that exerts the acceleration $a$ is equal to the mass $m$ that the acceleration $a$ is exerted on, and both masses are its own mass. This means that an inertial force is a kind of self-gravity. That is, an object receives gravity from its own gravitational field that moves in the same 
way as the object, and that gravity tries to keep the object in uniform linear motion. That gravity is an inertial force.

From these, we conclude that an inertial force is the same force as gravity and is a kind of self-gravity. Inertial mass is the mass that acceleration is exerted on, and an inertial force is a kind of self-gravity, so inertial mass is equivalent to passive gravitational mass.

However, this idea contains the following problem. Calculating gravity and an inertial force based on the gravity equation of thermodynamic gravity form, the spacetime particle distribution by gravity exists, and at the same time, the spacetime particle distribution by an inertial force exists. It is unnatural that there are some different distributions for one object at the same time. Therefore, the distribution by an inertial force is not a real distribution but a virtual distribution. In reality, the distribution by gravity should exert the inertial force.

We explain the relationship of mass in this paper.

Assumption 2.9: If an object has active gravitational mass, the active gravitational mass, passive gravitational mass and inertial mass of the object are all equal to mass $m$. Assumption 2.9 is the same as the equivalence principle.

Assumption 2.10: If an object has no active gravitational mass, the active gravitational mass of the object is zero, and the passive gravitational mass and inertial mass of the object are equal to the mass

$$
m=\frac{\mathrm{h} f}{\mathrm{c}^{2}},
$$

where $f$ is frequency. From assumption 2.10, in this paper, the absence of mass means that active gravitational mass is zero but passive gravitational mass and inertial mass are $m=\frac{\mathrm{h} f}{\mathrm{c}^{2}} \neq 0$. The reasons for assumption 2.10 are as follows. First, to move at the speed of light, active gravitational mass must be zero. Second, inertial mass is the mass that acceleration is exerted on, and an inertial force is a kind of self-gravity, so inertial mass is equivalent to passive gravitational mass. Third, if active gravitational mass is zero and passive gravitational mass and inertial mass exists, we can explain that light is bent by gravity while moving at the speed of light. Fourth, the rest energy of matter is expressed as

$$
E=m c^{2}
$$

and the energy of light is expressed as

$$
E=\mathrm{h} f \text {. }
$$

The matter having the rest energy $E=m \mathrm{c}^{2}$ is produced by the energy $E=\mathrm{h} f$ of light, and conversely, the light having the energy $E=\mathrm{h} f$ is produced by the annihilation of matter having the rest energy $E=m c^{2}$. Therefore, the energy $E$ is expressed as 


$$
E=m c^{2}=\mathrm{h} f .
$$

This suggests that the object with the energy of $E=\mathrm{h} f$ has the mass of $m=\frac{\mathrm{h} f}{\mathrm{c}^{2}}$. From these, we conclude assumption 2.10.

We explain the mass and speed of spacetime particles. From the general theory of relativity, gravitational waves propagate at the speed of light and should be carried by gravitons. Therefore, spacetime particles, which are gravitons, must move at the speed of light. However, spacetime particles have mass. From assumption 2.7, the distribution of spacetime particles is determined only by the mass of ordinary matter, so the spacetime particle number density does not change depending on the mass of spacetime particles. That is, spacetime particles do not have active gravitational mass that generates the spacetime particle number density $\frac{\mathrm{d} N}{\mathrm{~d} V}$. Therefore, from assumption 2.10, the active gravitational mass of the spacetime particle is zero, and the passive gravitational mass and inertial mass of the spacetime particle are $m_{s p}$. Since spacetime particles do not have active gravitational mass, they move at the speed of light, even if they have mass.

\section{The Gravity of Spacetime Particles That Are Dark Matter}

In this section, we discuss the gravity of spacetime particles that are dark matter. We derive the equation of gravity of spacetime particles, we calculate the basic mass of spacetime particles from observation results, and we compare the distance characteristics of gravity of ordinary matter and spacetime particles. With the gravity of spacetime particles, we solve the galaxy rotation problem.

\subsection{The Gravity of Spacetime Particles}

We derive equations associated with the gravity of spacetime particles.

Since one spacetime particle has the mass $m_{s p}$, from equation (2.15), the mass $m_{s p} N$ of $N$ spacetime particles is expressed as

$$
m_{s p} N=\frac{M r}{\mathrm{r}_{\mathrm{e}}},
$$

where $\mathrm{r}_{\mathrm{e}}$ is the distance expressed as

$$
\mathrm{r}_{\mathrm{e}}=\frac{\mathrm{k}_{\mathrm{sp}} T_{s p}}{m_{s p}} .
$$

From equation (3.1), Newtonian gravity $F$ of $N$ spacetime particles is expressed as 


$$
F=-\frac{\mathrm{G} M m}{r \times \mathrm{r}_{\mathrm{e}}} .
$$

From equation (3.3), gravitational acceleration $a$ of $N$ spacetime particles is expressed as

$$
a=-\frac{\mathrm{G} M}{r \times \mathrm{r}_{\mathrm{e}}} .
$$

From equations (2.5) and (3.4), the Unruh temperature $T_{u}$ by $N$ spacetime particles is expressed as

$$
T u=-\frac{\mathrm{k}_{\mathrm{sp}}}{\mathrm{k}_{\mathrm{B}}} \times \frac{\mathrm{G} M}{r \times \mathrm{r}_{\mathrm{e}}} .
$$

From equations (2.4) and (3.5), the temperature $T$ by $N$ spacetime particles is expressed as

$$
T=-\frac{m}{M} \times T_{s p} \times \frac{\mathrm{k}_{\mathrm{sp}}}{\mathrm{k}_{\mathrm{B}}} \times \frac{\mathrm{G} M}{r \times \mathrm{r}_{\mathrm{e}}}
$$

From equations (2.3) and (2.9) and (3.3) and (3.6), the spacetime particle number density $\frac{\mathrm{d} N}{\mathrm{~d} V}$ by $N$ spacetime particles is expressed as

$$
\frac{\mathrm{d} N}{\mathrm{~d} V}=\frac{M}{4 \pi r^{2} \mathrm{k}_{\mathrm{sp}} T_{s p}} .
$$

Therefore, equation (3.7) equal to the spacetime particle number density by the mass $M$ of ordinary matter, and we can understand that the mass of $N$ spacetime particles does not affect the spacetime particle distribution. Since the general equation of the spacetime particle number density $\frac{\mathrm{d} N}{\mathrm{~d} V}$ is expressed by equation (2.14), if the area $S$ where acceleration $a$ is constant is the same, the spacetime particle number densities are all the same. In this way, equations associated with the gravity of spacetime particles are derived.

\subsection{The Gravity of Ordinary Matter and $N$ Spacetime Particles}

We show the equations of gravity of ordinary matter and $N$ spacetime particles in Newtonian gravity form and thermodynamic gravity form.

First, we show the equations of gravity in Newtonian gravity form. From equation (2.10), the gravity $F_{1}$ of ordinary matter is expressed as

$$
F_{1}=-\frac{\mathrm{G} M m}{r^{2}}
$$

From equation (3.3), the gravity $F_{2}$ of $N$ spacetime particles is expressed as 


$$
F_{2}=-\frac{\mathrm{G} M m}{r \times \mathrm{r}_{\mathrm{e}}} .
$$

Therefore, the gravity $F$ of ordinary matter and $N$ spacetime particles is expressed as

$$
F=F_{1}+F_{2}=-\frac{\mathrm{G} M m}{r^{2}}-\frac{\mathrm{G} M m}{r \times \mathrm{r}_{\mathrm{e}}} .
$$

Second, we show the equations of gravity in thermodynamic gravity form. From equations (2.9) and (2.12), the gravity $F_{1}$ of ordinary matter is expressed as

$$
F_{1}=-S \times \frac{\mathrm{d} N}{\mathrm{~d} V} \times \mathrm{k}_{\mathrm{B}} \times \frac{m}{M} \times T_{s p} \times \frac{\mathrm{k}_{\mathrm{sp}}}{\mathrm{k}_{\mathrm{B}}} \times \frac{\mathrm{G} M}{r^{2}} .
$$

From equations (2.9) and (3.6), the gravity $F_{2}$ of $N$ spacetime particles is expressed as

$$
F_{2}=-S \times \frac{\mathrm{d} N}{\mathrm{~d} V} \times \mathrm{k}_{\mathrm{B}} \times \frac{m}{M} \times T_{s p} \times \frac{\mathrm{k}_{\mathrm{sp}}}{\mathrm{k}_{\mathrm{B}}} \times \frac{\mathrm{G} M}{r \times \mathrm{r}_{\mathrm{e}}} .
$$

Therefore, the gravity $F$ of ordinary matter and $N$ spacetime particles is expressed as

$$
F=F_{1}+F_{2}=-S \times \frac{\mathrm{d} N}{\mathrm{~d} V} \times \mathrm{k}_{\mathrm{B}} \times \frac{m}{M} \times T_{s p} \times \frac{\mathrm{k}_{\mathrm{sp}}}{\mathrm{k}_{\mathrm{B}}} \times\left(\frac{\mathrm{G} M}{r^{2}}+\frac{\mathrm{G} M}{r \times \mathrm{r}_{\mathrm{e}}}\right) .
$$

From equations (3.10) and (3.13), the gravity $F$ is expressed as

$$
F=-\frac{\mathrm{G} M m}{r^{2}}-\frac{\mathrm{G} M m}{r \times \mathrm{r}_{\mathrm{e}}}=-S \times \frac{\mathrm{d} N}{\mathrm{~d} V} \times \mathrm{k}_{\mathrm{B}} \times \frac{m}{M} \times T_{s p} \times \frac{\mathrm{k}_{\mathrm{sp}}}{\mathrm{k}_{\mathrm{B}}} \times\left(\frac{\mathrm{G} M}{r^{2}}+\frac{\mathrm{G} M}{r \times \mathrm{r}_{\mathrm{e}}}\right) .
$$

From equation (3.14), the gravity of $N$ spacetime particles does not affect the distribution of spacetime particles and exerts gravity in the form of an increase in temperature. In this way, the gravity of ordinary matter and $N$ spacetime particles is expressed.

\subsection{Calculation of the Basic Mass of Spacetime Particles}

We calculate the basic mass of spacetime particles from observation data.

First of all, we show the equation of the basic mass of spacetime particles. From equations (3.1) and (3.2), we can calculate $m_{s p}$ with the following equation:

$$
m_{s p}=m_{s p} N \times \frac{\mathrm{k}_{\mathrm{sp}} T_{s p}}{M r} .
$$

However, since the value of $T_{s p}$ is not known at this stage, we calculate the following mass: 


$$
\mathrm{m}_{\mathrm{sp} 0}=\frac{m_{s p}}{T_{s p}}=m_{s p} N \times \frac{\mathrm{k}_{\mathrm{sp}}}{M r}
$$

The mass $\mathrm{m}_{\mathrm{sp} 0}$ is the basic mass of spacetime particles. In section 5 , the value of $T_{s p}$ is derived as the function of the spin quantum number. From equation (3.16), we can calculate the basic mass $\mathrm{m}_{\mathrm{sp} 0}$ of spacetime particles if we know the three elements: the mass $M$ of ordinary matter, the radius $r$ from the mass $M$, and the mass $m_{s p} N$ of dark matter (spacetime particles).

We show data for $r, M, m_{s p} N$ and so on.

First, we show data for $m_{s p} N$. Studies indicate that the mass of dark matter in the Milky Way ranges from $4.5 \times 10^{12} \mathrm{M}_{\odot}[9]$ to $8 \times 10^{11} \mathrm{M}_{\odot}[10]$. We take the average value of these and assume the mass $m_{s p} N$ of dark matter (spacetime particles) as follows.

Assumption 3.1:

$$
m_{s p} N=\frac{4.5 \times 10^{12}+8 \times 10^{11}}{2}=2.65 \times 10^{12} \mathrm{M}_{\odot} .
$$

Second, we show data for $M$. Studies estimate that the total mass of all the stars in the Milky Way is between $4.6 \times 10^{10} \mathrm{M}_{\odot}[11]$ and $6.43 \times 10^{10} \mathrm{M}_{\odot}$ [12]. We take the average value of these and assume the mass $M$ of ordinary matter as follows.

Assumption 3.2:

$$
M=\frac{4.6 \times 10^{10}+6.43 \times 10^{10}}{2}=5.515 \times 10^{10} \mathrm{M}_{\odot} .
$$

Third, we show the orbital speed in the Milky Way. According to the recommended value of the International Astronomical Union, the orbital speed of stars at a certain distance from the Galactic Center is approximately $220 \mathrm{~km} / \mathrm{s}$. The orbital speed of the Sun is also approximately $220 \mathrm{~km} / \mathrm{s}$. The rotational speed calculated from the basic mass of spacetime particles must be equal to this orbital speed. For this reason, we assume as follows.

Assumption 3.3: The distance between the center of the Milky Way and the Sun is used for the radius $r$ of the calculation of $\mathrm{m}_{\mathrm{sp} 0}$, and $\frac{m_{s p} N}{M}=48.05$ holds at that radius $r$.

The value of 48.05 in assumption 3.3 is derived from $m_{s p} N$ in equation (3.17) and $M$ in equation (3.18). Assuming assumption 3.3, the rotational speed at the radius $r$ is calculated to be $220 \mathrm{~km} / \mathrm{s}$. Precisely, $r$ is the distribution radius of $m_{s p} N$ in equation (3.17). However, since the exact value of the radius is not known and the rotational speed needs to be 220 $\mathrm{km} / \mathrm{s}$, we assume assumption 3.3.

Fourth, we show data for $r$. From assumption 3.3, the radius $r$ is the distance between the center of the Milky Way and the Sun. Studies estimate that the distance between the center of the Milky Way and the Sun is $8.32 \mathrm{kpc}$, or $2.71 \times 10^{4}$ light-years [13]. Therefore, the radius $r$ is expressed as 


$$
r=8.32 \mathrm{kpc}=2.71 \times 10^{4} \text { light }- \text { years } .
$$

We calculate the mass $\mathrm{m}_{\mathrm{sp} 0}$ and the Compton wavelength. From equation (3.16) and these values, the mass $\mathrm{m}_{\mathrm{sp} 0}$ is calculated as

$$
\mathrm{m}_{\mathrm{sp} 0}=\frac{m_{s p}}{T_{s p}}=m_{s p} N \times \frac{\mathrm{k}_{\mathrm{sp}}}{M r}=1.05 \times 10^{-62} \mathrm{~kg} .
$$

Since the spacetime particle has the smallest mass, the mass $\mathrm{m}_{\mathrm{sp} 0}$ is extremely small. The Compton wavelength $\lambda$ is expressed as

$$
\lambda=\frac{\mathrm{h}}{m \mathrm{c}},
$$

where $\mathrm{h}$ is Planck constant. From equation (3.21), the Compton wavelength $\lambda_{0}$ at the mass $\mathrm{m}_{\mathrm{sp} 0}$ is expressed as

$$
\lambda_{0}=2.11 \times 10^{20} \text { meters } .
$$

In this way, the basic mass of spacetime particles is calculated as $1.05 \times 10^{-62} \mathrm{~kg}$. Note that the calculation of the basic mass of spacetime particles is not based on reliable data.

\subsection{Comparison of the Distance Characteristics of Gravity of Ordinary Matter and Spacetime Particles}

We compare the distance characteristics of gravity of ordinary matter and spacetime particles.

First of all, we show the distance where the gravity of ordinary matter and the gravity of $N$ spacetime particles are equal. From equations (3.8) and (3.9), that distance $r$ is expressed as $r_{e}$. Therefore, the distance $r_{e}$ of equation (3.2) means the distance where the gravity of ordinary matter and the gravity of $N$ spacetime particles are equal. From equations (3.2) and (3.16), the distance $r_{e}$ is expressed as

$$
\mathrm{r}_{\mathrm{e}}=\frac{\mathrm{k}_{\mathrm{sp}}}{\mathrm{m}_{\mathrm{sp} 0}} .
$$

Therefore, the distance $r_{e}$ is expressed as a constant. From equations (2.6) and (3.20), the concrete value of $r_{e}$ is expressed as

$$
\mathrm{r}_{\mathrm{e}}=5.34 \times 10^{18} \text { meters }=565 \text { light }- \text { years } .
$$

We show an approximate equation of gravity for each distance. The gravity $F$ of ordinary matter and $N$ spacetime particles is expressed by equation (3.10). When we rearrange equation (3.10), it is expressed as 


$$
F=-\frac{\mathrm{G} M m}{r} \times\left(\frac{1}{r}+\frac{1}{\mathrm{r}_{\mathrm{e}}}\right)
$$

- Case 1: $\mathrm{r}_{\mathrm{e}}>>r$. From $\frac{1}{r}>>\frac{1}{\mathrm{r}_{\mathrm{e}}}$, equation (3.25) can be approximated as

$$
F \fallingdotseq-\frac{\mathrm{G} M m}{r^{2}}
$$

- Case 2: $\mathrm{r}_{\mathrm{e}}<<r$. From $\frac{1}{r}<<\frac{1}{\mathrm{r}_{\mathrm{e}}}$, equation (3.25) can be approximated as

$$
F \fallingdotseq-\frac{\mathrm{G} M m}{r \times \mathrm{r}_{\mathrm{e}}}
$$

From these, we conclude as follows. At a short distance of $r_{e}>>r$, the gravity of ordinary matter becomes dominant, and the gravity of dark matter does not affect it. At a long distance of $\mathrm{r}_{\mathrm{e}}<<r$, the gravity of dark matter becomes dominant, and the gravity of ordinary matter does not affect it.

\subsection{Rotation Speed of Galaxies}

We calculate the rotation speed of galaxies.

The gravity $F$ of ordinary matter and $N$ spacetime particles is expressed by equation (3.10). When an object moves in uniform circular motion, the acceleration $a$ in the center direction of a circle is expressed as

$$
a=-\frac{v^{2}}{r}
$$

where $v$ is the rotation speed of the object, and $r$ is the radius from the center. The minus sign represents the direction of the center. Since gravity is equal to the force by the acceleration in the central direction, the equation of motion of the object is expressed as

$$
-\frac{m v^{2}}{r}=-\frac{\mathrm{G} M m}{r^{2}}-\frac{\mathrm{G} M m}{r \times \mathrm{r}_{\mathrm{e}}}
$$

From equation (3.29), the rotation speed $v$ is expressed as

$$
v=\sqrt{\mathrm{G} M \times\left(\frac{1}{r}+\frac{1}{\mathrm{r}_{\mathrm{e}}}\right)} .
$$

If $\mathrm{r}_{\mathrm{e}}<<r$, then $\frac{1}{r}<<\frac{1}{\mathrm{r}_{\mathrm{e}}}$, so equation (3.30) can be approximated as 


$$
v \fallingdotseq \sqrt{\frac{\mathrm{G} M}{\mathrm{r}_{\mathrm{e}}}} .
$$

From equation (3.31), in the case of $\mathrm{r}_{\mathrm{e}}<<r$, the rotation speed of galaxies is constant regardless of the radius $r$. Therefore, we can explain the galaxy rotation problem that the rotation speed becomes almost constant above a certain distance.

As an example, we calculate the rotation speed of the Milky Way. From equation (3.1), equation (3.29) is expressed as

$$
-\frac{v^{2}}{r}=-\mathrm{G} \frac{\left(M+m_{s p} N\right)}{r^{2}} .
$$

As mentioned earlier, the orbital speed $v$ of the Sun is approximately $220 \mathrm{~km} / \mathrm{s}$, and the distance $r$ between the center of the Milky Way and the Sun is $8.32 \mathrm{kpc}$, or $2.71 \times 10^{4}$ lightyears. From assumption $3.3, \frac{m_{s p} N}{M}=48.05$ holds for that radius $r$. Therefore, from equation (3.32) and these values, the mass $M$ of ordinary matter within the radius $r$ can be calculated as

$$
M=3.80 \times 10^{39} \mathrm{~kg} .
$$

Using the mass $M$ of equation (3.33), we show graphs of equations (3.30) and (3.31) in figure 1. As we can understand from the figure, the rotation speed becomes almost constant at approximately $220 \mathrm{~km} / \mathrm{s}$ from approximately 10,000 light-years.

In this way, we can solve the galaxy rotation problem by the gravity of spacetime particles. 


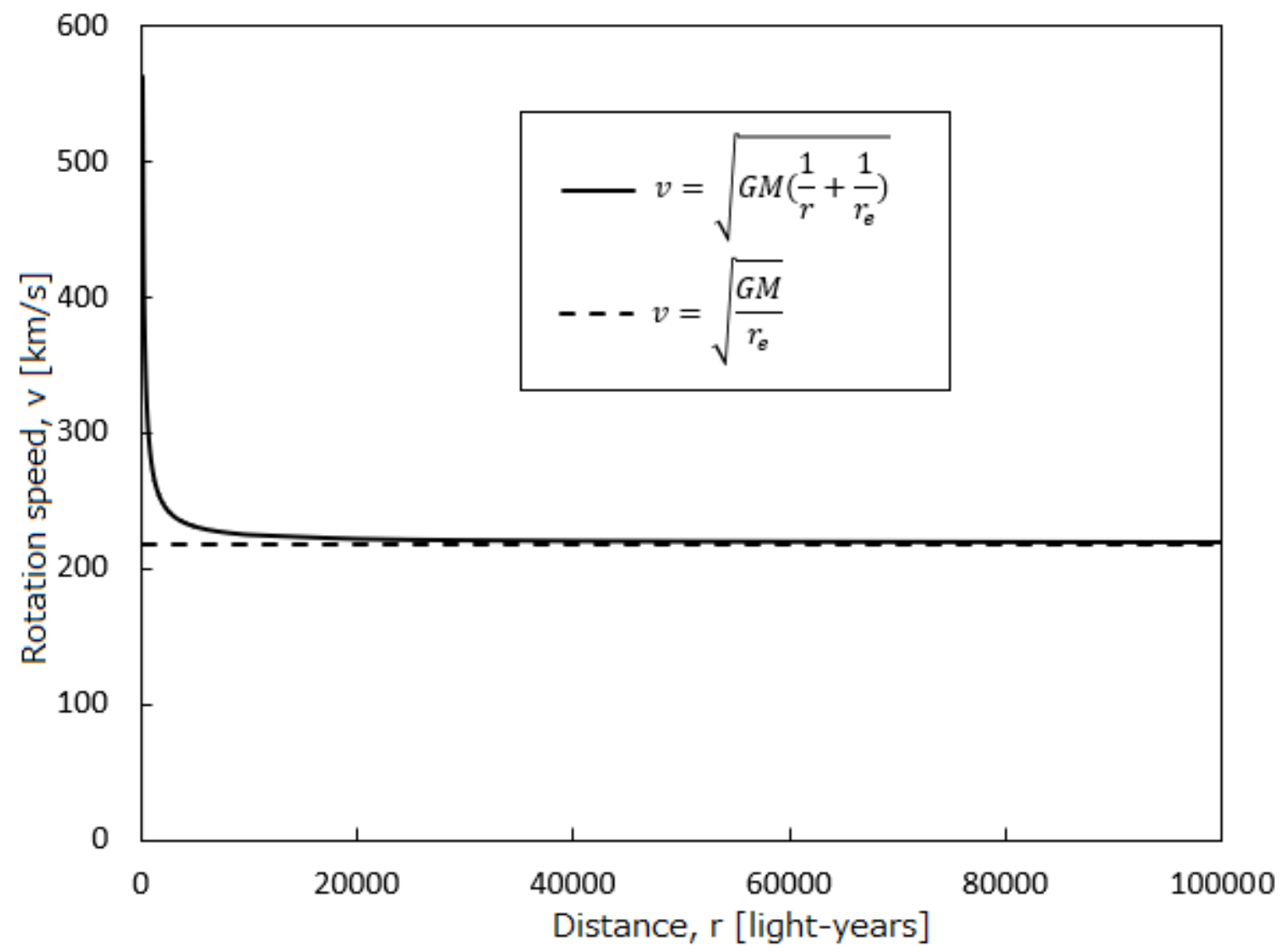

Figure 1: Graphs of the rotation speed $v$ of the object located at the distance $r$ from the mass $M$. There is a mass point with mass $M=3.80 \times 10^{39} \mathrm{~kg}$ in the center. The rotation speed $v=\sqrt{\mathrm{G} M \times\left(\frac{1}{r}+\frac{1}{\mathrm{r}_{\mathrm{e}}}\right)}$ is the rotation speed when receiving the gravity $F_{1}=-\frac{\mathrm{G} M m}{r^{2}}$ of ordinary matter and the gravity $F_{2}=-\frac{\mathrm{G} M m}{r \times \mathrm{r}_{\mathrm{e}}}$ of $N$ spacetime particles. The rotation speed $v=\sqrt{\frac{\mathrm{G} M}{\mathrm{r}_{\mathrm{e}}}}$ is the rotation speed when receiving only the gravity $F_{2}=-\frac{\mathrm{G} M m}{r \times \mathrm{r}_{\mathrm{e}}}$ of $N$ spacetime particles.

\section{The Shape of Spacetime Particles and Quantization by Spacetime Particles}

In this section, we discuss the shape of spacetime particles and the quantization of spacetime, gravity, mass, and energy by spacetime particles. From the shape of spacetime particles, we explain the structure of spacetime. 


\subsection{The Shape of Spacetime Particles and Structure of Spacetime}

We show the shape of spacetime particles and the structure of spacetime.

From equations (2.13) and (2.18), we set the distance $\Delta r$ as

$$
\Delta r=\frac{\mathrm{k}_{\mathrm{sp}} T_{s p}}{M} .
$$

From equation (4.1), equation (2.13) is expressed as

$$
\frac{\mathrm{d} N}{\mathrm{~d} V}=\frac{1}{4 \pi r^{2} \times \Delta r},
$$

and equation (2.18) is expressed as

$$
r=N \Delta r
$$

The radius $r$ of equation (4.3) represents the radius of each spacetime particle, and the distance $\Delta r$ of equation (4.1) represents the distance between spacetime particles. Therefore, the distance $\Delta r$ represents the quantized minimum distance in the spacetime radius. The spacetime radius is discretized for each $\Delta r$ and is quantized by spacetime particles.

The numerator of equation (4.2) represents one spacetime particle number, and the denominator of that represents the volume of one spacetime particle. Therefore, the volume $\Delta V$ of one spacetime particle is expressed as

$$
\Delta V=4 \pi r^{2} \times \Delta r .
$$

The volume $\Delta V$ is the surface area of a sphere at radius $r$ multiplied by the quantized minimum distance $\Delta r$. From equation (4.4), spacetime particles are spherical surface particles that are hollow and distributed only on the surface of the sphere.

Since such spacetime particles are distributed at every radius $r=N \Delta r(N=1,2,3 \ldots)$ from mass $M$, the structure of spacetime is the nested structure of spherical surface particles, as shown in figure 2 . 


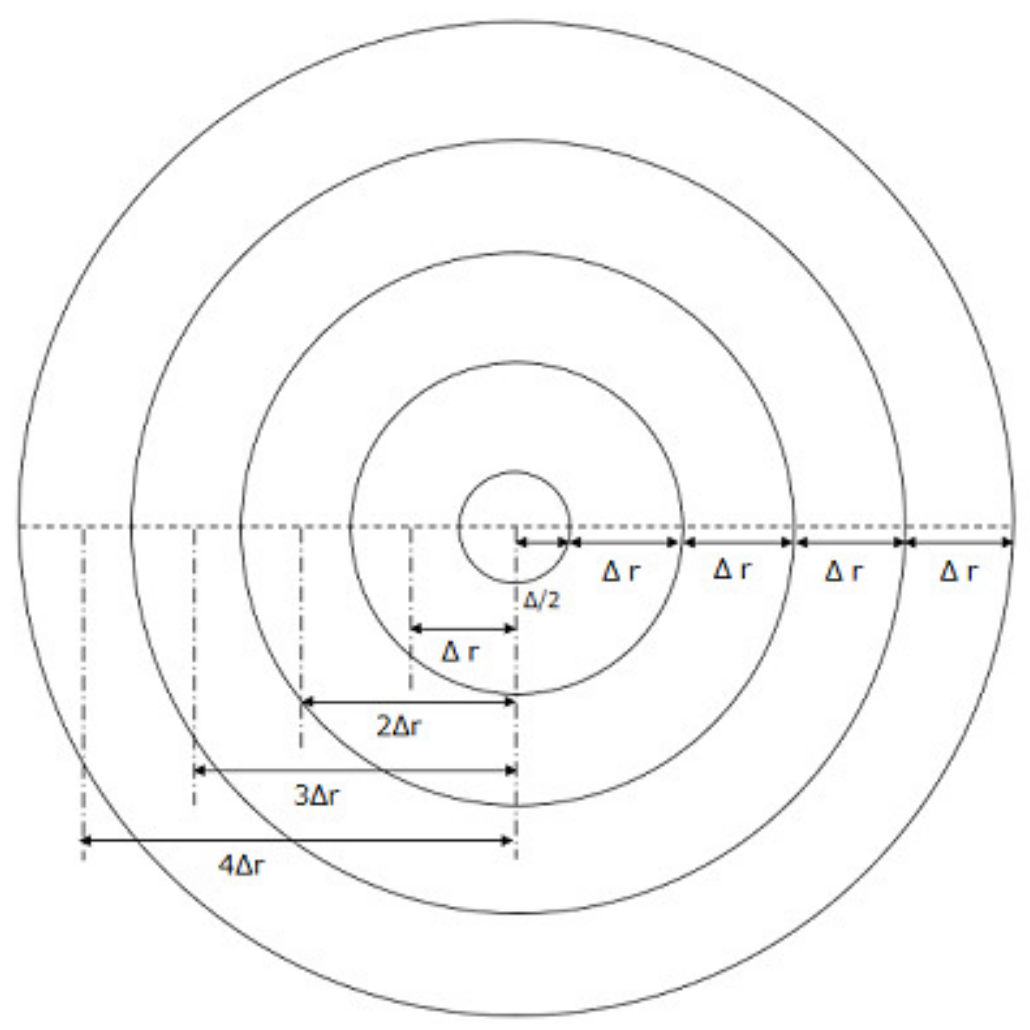

Figure 2: Spacetime particle distribution (spacetime structure) around elementary particles. There is an elementary particle with mass $M$ in the center, and spacetime particles with a volume of $\Delta V=4 \pi r^{2} \times \Delta r$ are distributed at every radius $r=N \Delta r(N=1,2,3 \ldots)$ from the elementary particle. Figure 2 represents the structure of spacetime because a group of spacetime particles is spacetime. The distance $\Delta r$ is the quantized minimum distance.

When we consider only the surface of the sphere with $r=N \Delta r$, the structure of spacetime is expressed as

$$
x^{2}+y^{2}+z^{2}=r^{2},
$$

where $x, y$ and $z$ are the coordinates of the $\mathrm{x}$-axis, $\mathrm{y}$-axis and $\mathrm{z}$-axis in Cartesian coordinates. If $r$ is constant, equation (4.5) becomes a 2-dimensional sphere. However, from equation (4.3), $r$ is not constant and is the variable of the spacetime particle number $N$.

Here, we define the spacetime particle number $N$ as an integer value in the range $0 \leqq$ $N \leqq N_{\max }\left(N=0,1,2 \ldots N_{\max }\right)$. Then we consider $N_{\max }-N$, which has the range $0 \leqq$ $N_{\max }-N \leqq N_{\max }\left(N_{\max }-N=0,1,2 \ldots N_{\max }\right)$. In this case, radius $r$ can be expressed as 


$$
r=\left(N_{\max }-N\right) \Delta r
$$

From equation (4.6), the square $r^{2}$ of radius is expressed as

$$
r^{2}=\left(N_{\max }^{2}-2 N \times N_{\max }+N^{2}\right) \Delta r^{2} .
$$

From equation (4.7), we set $r_{\max }$ as

$$
r_{\max }=N_{\max } \Delta r
$$

and we set $r_{n}$ as

$$
r_{n}=\sqrt{2 N \times N_{\max }-N^{2}} \times \Delta r\left(0 \leqq r_{n} \leqq r_{\max }\right) .
$$

From equations (4.8) and (4.9), the square $r^{2}$ of radius is expressed as

$$
r^{2}=r_{\max }^{2}-r_{n}^{2} .
$$

Therefore, equation (4.5) is expressed as

$$
x^{2}+y^{2}+z^{2}+r_{n}^{2}=r_{\text {max }}^{2} .
$$

The max radius $r_{\max }$ is a constant, and the radius $r_{n}$ is the variable of the spacetime particle number $N$ and changes discretely between 0 and $r_{\max }$. Therefore, equation (4.11) represents a quantized 3-dimensional sphere. That is, the structure of spacetime is the quantized 3-dimensional sphere of Poincaré conjecture.

In this way, since spacetime particles are represented as spherical surface particles, the structure of spacetime is represented as the quantized 3-dimensional sphere of Poincaré conjecture.

\subsection{Thermal Vibration by the Dimensionless Temperature $T_{s p}$}

We discuss the thermal vibration by the dimensionless temperature $T_{s p}$.

The element $T_{s p}$ in the equation of state of spacetime particles corresponds to the temperature $T$ in the ideal gas law, and it is dimensionless. Therefore, the element $T_{s p}$ can be regarded as the dimensionless temperature of spacetime particles.

The average kinetic energy of gas molecules is expressed as

$$
\frac{m \bar{v}^{2}}{2}=\frac{3}{2} \mathrm{k}_{\mathrm{B}} T
$$

where $\bar{v}$ is the average speed of gas molecules. From equation (4.1), an equation similar to equation (4.12) is shown as 


$$
M \Delta r=\mathrm{k}_{\mathrm{sp}} T_{s p} .
$$

The element $\mathrm{k}_{\mathrm{B}} T$ represents that the particles in space are moving with the average kinetic energy corresponding to the temperature. Since the equation of state of spacetime particles has a similar form as the ideal gas law, consider $\mathrm{k}_{\mathrm{sp}} T_{s p}$ in the same way as $\mathrm{k}_{\mathrm{B}} T$. Then, the element $\mathrm{k}_{\mathrm{sp}} T_{s p}$ should represent that spacetime particles in space are moving with the physical quantity of $M \Delta r$ corresponding to the temperature $T_{s p}$. From the structure of spacetime of the quantized 3-dimensional sphere, specifically, the radius of spacetime particles are moving only in the radial direction in the space between spacetime particles of $\Delta r$. That is, the radius of spacetime particles repeatedly contracts and expands in the radial direction, and it thermally oscillates in the space of $\Delta r$ between spacetime particles.

Conversely, since the entire spherical surface of spacetime particles thermally oscillates by $\Delta r$ in the radial direction, the distance between spacetime particles is separated by $\Delta r$. For this reason, the higher $T_{s p}$ is, the larger the distance $\Delta r$ between spacetime particles becomes, and the lower $T_{s p}$ is, the smaller the distance $\Delta r$ between spacetime particles becomes. Therefore, spacetime particles have a radial width of $\Delta r$ by the thermal vibration of the dimensionless temperature $T_{s p}$.

Each spacetime particle has a radius of $r=N \Delta r$ and has a width of $\Delta r$ in the radial direction by the thermal vibration of $T_{s p}$. Therefore, we conclude that each spacetime particle is a harmonic oscillator and thermally oscillates with $r=N \Delta r$ as a fixed point, and its radius $r$ thermally oscillates in the following range:

$$
N \Delta r-\frac{\Delta r}{2}<r \leqq N \Delta r+\frac{\Delta r}{2} \quad(N=1,2,3 \ldots) .
$$

Spacetime or gravitational fields are a group of such harmonic oscillators.

We can explain why $T_{s p}$ has the dimension of dimensionless temperature as follows. The thermal vibration by general temperature is the change in the position of an object. The thermal vibration by $T_{s p}$ is the change in the radius of the object without changing its position. Since the position of the spacetime particle does not change even if its radius changes, the thermal vibration by $T_{s p}$ does not have translational kinetic energy. Therefore, the element $T_{s p}$ has the dimension of dimensionless temperature, which is different from the general temperature.

In this way, the thermal vibration by $T_{s p}$ is a radial motion, and spacetime particles have a radial width of $\Delta r$ by the thermal vibration of $T_{s p}$, and they are harmonic oscillators by the thermal vibration of $T_{s p}$. 


\subsection{Uncertainty Principle of Spacetime Particle}

From the quantized minimum distance $\Delta r$ of equation (4.1), we derive an equation equivalent to the uncertainty principle.

We derive $\Delta E \times \Delta t=\frac{\hbar}{2}$. From the theory of relativity, energy $E$ is expressed as

$$
E=M \mathrm{c}^{2}
$$

From equations (2.6) and (4.15), equation (4.1) is expressed as

$$
E \times \frac{\pi}{T_{s p}} \times \frac{\Delta r}{\mathrm{c}}=\frac{\hbar}{2} .
$$

From equation (4.16), we set the time $\Delta t$ as

$$
\Delta t=\frac{\Delta r}{\mathrm{c}}=\frac{\mathrm{k}_{\mathrm{sp}} T_{s p}}{M \mathrm{c}}
$$

and we set the energy $\Delta E$ as

$$
\Delta E=E \times \frac{\pi}{T_{s p}}
$$

From equations (4.17) and (4.18), equation (4.16) is expressed as

$$
\Delta E \times \Delta t=\frac{\hbar}{2}
$$

Therefore, from the quantized minimum distance $\Delta r$ of equation (4.1), we can obtain the equation equivalent to the uncertainty principle between energy and time.

We derive $\Delta r \times \Delta p=\frac{\hbar}{2}$. From equation (2.6), equation (4.1) is expressed as

$$
\Delta r \times M c \times \frac{\pi}{T_{s p}}=\frac{\hbar}{2} .
$$

From equation (4.20), we set the momentum $\Delta p$ as

$$
\Delta p=M c \times \frac{\pi}{T_{s p}} .
$$

From equation (4.21), equation (4.20) is expressed as

$$
\Delta r \times \Delta p=\frac{\hbar}{2}
$$

Therefore, from the quantized minimum distance $\Delta r$ of equation (4.1), we can obtain the equation equivalent to the uncertainty principle between position and momentum. 
Since these equations are derived from the equation of state of spacetime particles, they are the uncertainty principle for spacetime particles moving at the speed of light around mass $M$. These equations also mean that the uncertainty of spacetime particles is determined based on the mass $M$ of the central object and the temperature $T_{s p}$. That is, the quantum fluctuation of spacetime is caused by the mass $M$ and the thermal vibration of $T_{s p}$. For these reasons, the velocity in the momentum is the speed of light, and the mass is not the mass $m_{s p}$ of the spacetime particle but the maximum mass $M$ allowed by the uncertainty principle.

From these equations, the distance $\Delta r$ represents the quantized minimum distance and at the same time represents the inaccuracy of the distance based on the uncertainty principle. The time $\Delta t$ and the distance $\Delta r$ represent the inaccuracy of time and distance, which cannot be measured more precisely than these. For this reason, when measuring time and distance, we can only measure in units of $\Delta t$ and $\Delta r$. Therefore, the inaccuracy $\Delta t$ of time and the inaccuracy $\Delta r$ of distance represent the quantized minimum time and distance.

From the above, the equation of state of spacetime particles is the equation of state based on the uncertainty principle, and the gravitational theory in this paper is the gravitational theory based on the uncertainty principle of quantum mechanics.

\subsection{Quantization Law of Space}

There is a certain law in the quantization of space by spacetime particles. We explain that law and quantize space based on that law.

First of all, we explain the quantization law of space. From the quantized minimum distance $\Delta r$ of equation (4.1), the spacetime particle number density of equation (2.14) is expressed as

$$
\frac{\mathrm{d} N}{\mathrm{~d} V}=\frac{1}{S \times \Delta r}
$$

From equation (4.23), the volume $\Delta V$ of spacetime particles for any area $S$ is expressed as

$$
\Delta V=S \times \Delta r
$$

The area $S$ is the area where force is constant. Since the distance $\Delta r$ is 1-dimensional space, the dimension of area $S$ is one lower than all spatial dimensions of force. Therefore, the relationship of spatial dimension is expressed as

$$
D_{r}=D_{s p}+1,
$$

where $D_{r}$ is the all spatial dimensions of force, and $D_{s p}$ is the spatial dimension where force is constant. From equation (4.24), the volume $V$ of Dr-dimensional space is quantized as 


$$
V=\sum \Delta V=\sum(S \times \Delta r)
$$

where $S$ is the area of Dsp-dimensional space, $\Delta r$ is the quantized minimum distance of 1-dimensional space. In this way, the quantization law of space of equation (4.26) exists.

From the quantization law of space, we quantize space. In the case of gravity, $D_{s p}$ is 2, $D_{r}$ is 3 , and the area $S$ is the surface area of a sphere. Therefore, from equation (4.26), the volume $V$ of 3 -dimensional space is quantized as

$$
V=\sum \Delta V=\sum\left(4 \pi r^{2} \times \Delta r\right)
$$

From equation (4.3), the volume $V$ is expressed as

$$
V=\sum\left(4 \pi r^{2} \times \Delta r\right)=4 \pi \Delta r^{3} \times \frac{N}{6} \times(2 N+1) \times(N+1) .
$$

If $N$ is sufficiently large, the volume $V$ can be approximated as

$$
V=\sum\left(4 \pi r^{2} \times \Delta r\right) \fallingdotseq \int_{0}^{r} 4 \pi r^{2} \mathrm{~d} r=\frac{4 \pi r^{3}}{3}
$$

From equation (4.29), if $N$ is sufficiently large, the volume $V$ can be approximated as the volume of continuous space.

In the case of the spacetime radius, we can consider that $D_{s p}$ is $0, D_{r}$ is 1 , and the area $S$ is a zero-dimensional point. Therefore, from equation (4.26), the spacetime radius $r$ is expressed as

$$
r=\sum \Delta r=N \Delta r
$$

If $N$ is sufficiently large, the spacetime radius $r$ can be approximated as

$$
r=\sum \Delta r \fallingdotseq \int_{0}^{r} \mathrm{~d} r=r .
$$

From equation (4.31), if $N$ is sufficiently large, the spacetime radius $r$ can be approximated as the spacetime radius of continuous space.

In this way, we can quantize space based on the quantization law of space, and an approximately continuous space is derived from the quantized space.

\subsection{Quantization of Time}

We quantize time from the quantization law of space and discuss time.

First of all, We quantize time. Time is represented in one dimension. If we apply the quantization law of space to time, in the case of time, we can consider that $D_{s p}$ is $0, D_{r}$ is 
1, and the area $S$ is a zero-dimensional point. Therefore, from equations (4.17) and (4.26), time $t$ is expressed as

$$
t=\sum \Delta t=N \Delta t
$$

where $\Delta t$ is the quantized minimum time that is defined by equation (4.17). If $N$ is sufficiently large, time $t$ can be approximated as

$$
t=\sum \Delta t \fallingdotseq \int_{0}^{t} \mathrm{~d} t=t .
$$

From equation (4.33), if $N$ is sufficiently large, time $t$ can be approximated as continuous time.

We explain the relationship between time and space. When we divide the radius $r$ of equation (4.30) by the speed of light, from equation (4.17), it is expressed as

$$
\frac{r}{\mathrm{c}}=N \times \frac{\Delta r}{\mathrm{c}}=N \Delta t=t .
$$

From equation (4.34), $N$ in time $t$ is the spacetime particle number. The relationship between time and space is expressed by equations (4.17) and (4.34). From equations (4.17) and (4.34), time is closely related to space, and time and space cannot be separated and quantized individually. Therefore, time and space need to be unified in the form of spacetime.

We discuss the passage of time. Equation (4.34) leads us to conclude that the passage of time is equivalent to an increase in the spacetime particle number in space. Then, before the birth of the universe, there are no spacetime particles in space, so there is no passage of time. After the birth of the universe, the spacetime particle number in space increases, so time has passed. That is, since time and space are equivalent, the passage of time is equivalent to the expansion of space, and space always expands with the passage of time. Therefore, we conclude that if time continues to pass, space will also continue to expand.

We discuss the difference in how time advances. If it is the same time, the spacetime particle number $N$ in spacetime should be the same in different spacetime. Conversely, the same time means that the spacetime particle number $N$ in spacetime is the same. Therefore, we conclude that even if the spacetime particle number $N$ increases in the same way, the reason why time progresses differently is that the quantized minimum time $\Delta t$ is different.

In this way, time is quantized from the quantization law of space, and time is explained.

\subsection{Equation of Equivalence of Energy and Mass and Spacetime}

We quantize mass and energy, and we show the equation of equivalence of energy and mass and spacetime. 
From assumption 2.1, energy and mass and spacetime are equivalent. This means that mass or energy can be converted into spacetime, and spacetime can be converted into mass or energy. From assumption 2.2, spacetime is quantized by spacetime particles with the minimum mass $m_{s p}$, and spacetime is a group of spacetime particles. From assumptions 2.1 and 2.2, mass or energy can be converted into spacetime that is a group of spacetime particles. For this reason, we conclude that mass or energy is quantized by spacetime particles. Therefore, the mass $m$ of any object is expressed as

$$
m=n_{\max } \times m_{s p},
$$

where $n_{\max }$ is the maximum number of spacetime particles within the object. In this paper, the spacetime particle number $n_{\max }$ represents spacetime. Therefore, equation (4.35) means that mass can be converted to spacetime, and spacetime can be converted to mass. Equation (4.35) also means that mass is quantized by the minimum mass $m_{s p}$ of spacetime particles.

From equation (4.35) and $E=m c^{2}$, the energy $E$ of any object is expressed as

$$
E=m c^{2}=n_{\max } \times m_{s p} c^{2} .
$$

In equation (4.36), $E, m$ and $n_{\max }$ represent energy, mass and spacetime. Therefore, equation (4.36) means that energy $E$ and mass $m$ and spacetime $n_{\max }$ are equivalent. Equation (4.36) also means that energy is quantized by the minimum energy $m_{s p} c^{2}$ of spacetime particles. In the theory of relativity, only energy and mass are equivalent, but in this paper, energy and mass and spacetime are equivalent.

In this way, mass and energy are quantized by spacetime particles, and the equation of equivalence of energy and mass and spacetime is shown.

\subsection{Graviton Coordinates and Quantization of Gravity}

We quantize gravity and explain the coordinates quantized by spacetime particles.

From the radius $r$ of equation (4.3), the gravity of equation (3.10) is quantized as

$$
F=-\frac{\mathrm{G} M m}{(N \Delta r)^{2}}-\frac{\mathrm{G} M m}{N \Delta r \times \mathrm{r}_{\mathrm{e}}} .
$$

We show the coordinates quantized by spacetime particles. First, each spacetime particle is a harmonic oscillator, and its radius $r$ thermally oscillates in the range of $N \Delta r-\frac{\Delta r}{2}<r \leqq$ $N \Delta r+\frac{\Delta r}{2}(N=1,2,3 \ldots)$ with $r=N \Delta r$ as a fixed point. Note that there are no spacetime particles in the space of $r \leqq \frac{\Delta r}{2}$ and $r>\left(N+\frac{1}{2}\right) \Delta r$. Second, from the quantized gravity of equation (4.37), the radius in space of $\Delta r$ remains constant at the radius of $r=N \Delta r$. Therefore, we conclude that the radius $r$ in gravity or gravity $F$ is as follows.

- Case 1: If $r \leqq \frac{\Delta r}{2}$, gravity $F$ is $F=0$. 
- Case 2: If $N \Delta r-\frac{\Delta r}{2}<r \leqq N \Delta r+\frac{\Delta r}{2}, r=N \Delta r$, and gravity $F$ is $F \neq 0$.

- Case 3: If $r>\left(N+\frac{1}{2}\right) \Delta r$, gravity $F$ is $F=0$.

In this way, we conclude that the coordinates within $\Delta r$ are the same radius $r$. Therefore, in the coordinates related to the graviton, one graviton can represent only one radius $r=N \Delta r$, and any space in the space of $\Delta r$ is all represented as one radius $r=N \Delta r$. The coordinates related to the graviton are called graviton coordinates. On the other hand, ordinary nonquantized coordinates are called real coordinates.

There are two things to note. First, from the uncertainty principle, the radius $r=N \Delta r$ is not always observed as the radius $r=N \Delta r$, and the radius $r$ has the inaccuracy of the distance of $\Delta r$. However, on average, the radius $r$ is expressed as $r=N \Delta r$. Second, in the space of $r \leqq \frac{\Delta r}{2}$ and $r>\left(N+\frac{1}{2}\right) \Delta r$, since there are no gravitons, gravity $F$ is $F=0$. For this reason, there is no singularity at $r=N \Delta r=0$ in the gravity of equation (4.37). Therefore, the maximum gravity $F_{\max }$ is expressed as

$$
F_{\max }=-\frac{\mathrm{G} M m}{(\Delta r)^{2}}-\frac{\mathrm{G} M m}{\Delta r \times \mathrm{r}_{\mathrm{e}}} \fallingdotseq-\frac{\mathrm{G} M m}{(\Delta r)^{2}} .
$$

We explain the properties of individual spacetime particles of graviton coordinates. When we consider gravity in terms of thermodynamic gravity, an object appears to be distributed on graviton coordinates. This is because, from equations (2.2) and (2.3), the gravity $F$ at radius $r$ is expressed as

$$
F=\text { spherical surface area at radius } \mathrm{r} \times \text { pressure per unit area at radius } \mathrm{r} .
$$

From equation (4.39), even if an object exists at a certain point on the spherical surface, the pressure is the pressure of the entire spherical surface. That is, the position of the object appears to exist on the entire spherical surface. However, it is obvious that the object does not exist on the entire spherical surface. Therefore, this means that in graviton coordinates, the object is in contact with the entire spherical surface of the spacetime particle, even if it is in contact with one point of that.

From this, in graviton coordinates, exerting a force to the spacetime particle is equivalent to exerting a force to the entire spherical surface of the spacetime particle. Conversely, the force exerted from spacetime particles is equivalent to the force exerted from the entire spherical surface of the spacetime particle. Therefore, each spacetime particle is the smallest space in graviton coordinates, and they can be regarded as if it were a rigid body or a zerodimensional point space. Since time $t$ is expressed as $t=\frac{r}{\mathrm{c}}=N \Delta t$, in graviton coordinates, like space, the time in the smallest space represents only one time.

From the above, we summarize the properties of graviton coordinates as follows. 
Minimum space properties: At least in the case of things related to gravitons, each spacetime particle in graviton coordinates is the minimum space. The minimum space means that even if an object touches a certain point in the space, the object touches the entire space, and the entire space is equivalent to a rigid body or a zero-dimensional point space.

More specifically, the minimum space properties are as follows. First, in graviton coordinates, even if a force is exerted to one point in the minimum space, the force is exerted to the entire minimum space. Second, in graviton coordinates, even if a force is exerted from one point in the minimum space, the force is exerted from the entire minimum space. Third, in graviton coordinates, the time in the minimum space does not vary from place to place and is the same for the entire minimum space.

From the minimum space properties, we conclude as follows.

First, even if an object exists at a certain point in real coordinates, in graviton coordinates the object exists in the entire minimum space. Therefore, the coordinates in graviton coordinates are different from the coordinates in real coordinates. That is, at the same time, the object has different coordinates in different coordinates. For this reason, it is necessary to use the appropriate coordinates for the situation.

Second, in the case of things related to gravitons, it is physically meaningless to further divide the minimum space in graviton coordinates. This is because even if we try to cut out only a certain space in the minimum space, we will cut out the entire minimum space from the minimum space properties. Therefore, the minimum space that cannot be further divided is the space that has the minimum space properties.

Third, the nonlocality of quantum mechanics is brought about by the minimum space properties. This is because the nonlocality of quantum mechanics is equivalent to the minimum space properties.

In this way, gravity is quantized by spacetime particles, and the quantized graviton coordinates are explained.

\subsection{Summary of Quantization by Spacetime Particles}

In summary, the physical quantities quantized in this section are as follows.

- Quantization of mass : $m=n_{\max } \times m_{s p}$

- Quantization of energy : $E=n_{\max } \times m_{s p} \mathrm{c}^{2}$

- Quantization of radius : $r=N \Delta r, \Delta r=\frac{\mathrm{k}_{\mathrm{sp}} T_{s p}}{M}$

- Quantization of time : $t=N \Delta t, \Delta t=\frac{\mathrm{k}_{\mathrm{sp}} T_{s p}}{M \mathrm{c}}$ 
- Quantization of space : $V=\sum \Delta V=\sum\left(4 \pi r^{2} \times \Delta r\right)$

- Quantization of gravity : $F=-\frac{\mathrm{G} M m}{(N \Delta r)^{2}}-\frac{\mathrm{G} M m}{N \Delta r \times \mathrm{r}_{\mathrm{e}}}$

- Quantization of coordinates (Graviton coordinates):

If $r \leqq \frac{\Delta r}{2}$, gravity $F$ is $F=0$.

If $N \Delta r-\frac{\Delta r}{2}<r \leqq N \Delta r+\frac{\Delta r}{2}, r=N \Delta r$, and gravity $F$ is $F \neq 0$.

If $r>\left(N+\frac{1}{2}\right) \Delta r$, gravity $F$ is $F=0$.

In this way, all physical quantities related to energy and mass and spacetime are quantized by spacetime particles. The equations $t=N \Delta t$ and $r=N \Delta r$ mean that the time $t$ and the spacetime radius $r$ are equivalent to the spacetime particle number $N$, and we can understand that the spacetime particle number $N$ certainly represents time and space. These things are naturally derived from the contents up to section 2. Not we tried to quantize them, but we just realized that they were quantized when we considered gravity based on the quantum principle. That is, the gravitational theory in this paper is the gravitational theory originally based on quantum theory.

\section{Minimum Radius and Internal Structure of Elementary Particles}

In this section, we discuss the minimum radius and internal structure of elementary particles. Then we discuss the radius of protons and atomic nuclei. The expression " the internal structure of elementary particles" is strange as an expression of words because elementary particles are particles with no substructure. However, since this paper is just a hypothesis, if particles are generally considered to be elementary particles, we refer to their particles as elementary particles.

\subsection{Minimum Radius of Elementary Particles and Black Holes}

From the space of $0 \leqq r \leqq \frac{\Delta r}{2}$, we discuss the minimum radius of elementary particles and black holes.

First of all, we discuss the space of $0 \leqq r \leqq \frac{\Delta r}{2}$.

From equation (4.14), spacetime particles by an object's own active gravitational mass are not distributed in the space of $0 \leqq r \leqq \frac{\Delta r}{2}$. Spacetime particles are gravitons. Each spacetime particle is a harmonic oscillator, and its radius $r$ thermally oscillates in the range of $N \Delta r-\frac{\Delta r}{2}<r \leqq N \Delta r+\frac{\Delta r}{2}(N=1,2,3 \ldots)$ with $r=N \Delta r$ as a fixed point. When $N=1$, 
the spacetime particle thermally oscillates in the range of $\frac{\Delta r}{2}<r \leqq \frac{3 \Delta r}{2}$ with $r=\Delta r$ as a fixed point. Therefore, gravitons are not distributed in the space of $0 \leqq r \leqq \frac{\Delta r}{2}$.

From section 2, the particle carrying inertial forces is the graviton, and an inertial force is a kind of self-gravity. In the space of $0 \leqq r \leqq \frac{\Delta r}{2}$, since there is no graviton carrying gravity and inertial forces, gravity is zero and an inertial force does not work. Since an inertial force does not work, the object in the space of $0 \leqq r \leqq \frac{\Delta r}{2}$ moves at the speed of light. The object with active gravitational mass exists in the space of $0 \leqq r \leqq \frac{\Delta r}{2}$. Therefore, even if the object has active gravitational mass, it moves at the speed of light in the space of $0 \leqq r \leqq \frac{\Delta r}{2}$. For this reason, the object with active gravitational mass is equivalent to having no active gravitational mass in the space of $0 \leqq r \leqq \frac{\Delta r}{2}$. In this way, even if an object has mass, it moves at the speed of light in the space of $0 \leqq r \leqq \frac{\Delta r}{2}$.

Note that gravitons by the external active gravitational mass exist in the space of $0 \leqq$ $r \leqq \frac{\Delta r}{2}$. However, since an inertial force is a kind of self-gravity, the object moves at the speed of light in the space of $0 \leqq r \leqq \frac{\Delta r}{2}$.

We discuss the minimum radius of an object. In the space of $0 \leqq r \leqq \frac{\Delta r}{2}$, since gravitons by an object's own active gravitational mass are not distributed, the gravity that contracts the object does not work. If the repulsive force of the object works even a little, the object expands to $\frac{\Delta r}{2}$. Therefore, we conclude that the minimum radius $r_{0}$ of an object is expressed as

$$
r_{0}=\frac{\Delta r}{2}
$$

For example, in black holes, no matter how much an object contracts by gravity, it can only contract to the radius of $r=\frac{\Delta r}{2}$. Therefore, we conclude that the minimum radius of all objects, which includes objects in black holes and elementary particles, is $\frac{\Delta r}{2}$.

When observed, elementary particles are observed almost as point particles. However, the minimum radius is $\frac{\Delta r}{2}$. Therefore, we conclude that the radius of elementary particles at the time of observation is the minimum radius $\frac{\Delta r}{2}$.

If an object has the minimum radius $\frac{\Delta r}{2}$, it moves at the speed of light in the space of $\frac{\Delta r}{2}$ or less, and only its rotational speed is the speed of light. In fact, observations show that black holes are rotating at approximately the speed of light [14]. However, the rotational speed is not the perfect speed of light. This means that the radius of the object in the black hole is not the minimum radius. That is, since the radius of the object in the black hole is larger than the minimum radius, an inertial force works and its rotational speed is less than the speed of light.

Assuming that the minimum radius of an object is less than $\frac{\Delta r}{2}$, the following two problems arise. First, in the space of $\frac{\Delta r}{2}$ or less, the translational speed of an object with mass becomes the speed of light. This is problematic from the point of view of energy. As will be explained in section 9, if the rotational speed is the speed of light, it is no problem. Second, elementary 
particles have the spin angular momentum

$$
L=m v r
$$

However, when the radius of elementary particles is smaller than $\frac{\Delta r}{2}$, the rotational speed of elementary particles becomes faster than the speed of light. This means that the radius $r_{0}=\frac{\Delta r}{2}$ of elementary particles is the radius when the rotational speed based on the spin angular momentum is the speed of light. Therefore, we conclude that the minimum radius of an object is $\frac{\Delta r}{2}$.

In the case of an elementary particle without active gravitational mass, since there is no active gravitational mass, from the beginning there is no graviton by its own active gravitational mass. Therefore, not all of the discussions here apply. However, even in that case, for the rotational speed based on the spin angular momentum to be the speed of light, its radius must be $\frac{\Delta r}{2}$. Therefore, we conclude that elementary particles without active gravitational mass also have the minimum radius of $\frac{\Delta r}{2}$, and at least the radius at the time of observation is $\frac{\Delta r}{2}$.

From the spin angular momentum and the rotational speed at the speed of light, we calculate the minimum radius $r_{0}=\frac{\Delta r}{2}$ of elementary particles. Assuming that the mass $M$ of an elementary particle is in the radius $r_{0}$ as a mass point, the equation of the spin angular momentum is expressed as

$$
\hbar s=M v r_{0}
$$

where $s$ and $v$ is the spin quantum number and rotational speed of the elementary particle. The radius $r_{0}$ of the elementary particle at the time of observation is $r_{0}=\frac{\Delta r}{2}$. From equations (2.6) and (4.1) and (5.1) and (5.3), the rotational speed $v$ is expressed as

$$
v=\frac{4 \pi s}{T_{s p}} \times \mathrm{c} .
$$

Since an inertial force does not work in the space of $\frac{\Delta r}{2}$ or less, the rotational speed $v$ is the speed of light $(v=\mathrm{c})$. From equation (5.4) and $v=\mathrm{c}, T_{s p}$ is expressed as

$$
T_{s p}=4 \pi s
$$

Therefore, the minimum radius $r_{0}$ is expressed as

$$
r_{0}=\frac{\Delta r}{2}=\frac{\mathrm{k}_{\mathrm{sp}} \times 2 \pi s}{M} .
$$

As an example, we calculate the electron radius. From the mass $9.1093837015 \times 10^{-31}$ $\mathrm{kg}$ (2018 CODATA recommended values) and spin quantum number $1 / 2$ of an electron, the electron radius is expressed as 


$$
r_{0}=1.9307963398 \times 10^{-13} \text { meters . }
$$

From the Compton wavelength $\lambda$ of equation (3.21), the minimum radius $r_{0}$ is expressed as

$$
r_{0}=\frac{\Delta r}{2}=\frac{\lambda}{2 \pi} \times s
$$

The minimum radius $r_{0}$ at the spin quantum number 1 is expressed as

$$
r_{0}=\frac{\lambda}{2 \pi}=\frac{\mathrm{h}}{2 \pi M c} .
$$

Therefore, the minimum radius of equation (5.9) is equal to the range of a force. The range of a force represents the distance where the force can be carried. Gauge bosons carrying force have the spin quantum number of 1 except for specific particles. From these, the radius of the gauge boson is expressed as the minimum radius of equation (5.8), so we can explain that its range of a force becomes $\frac{\mathrm{h}}{2 \pi M c}$.

In this paper, gravitons have mass, but the range of gravity is infinite. This is because the radius of the graviton changes to infinity at the speed of light. Conversely, if the range of a force is finite, the radius of the particle carrying the force usually does not change and remains $\frac{\lambda}{2 \pi} \times s$. That is, the range $\frac{\mathrm{h}}{2 \pi M c}$ of a force represents the radius of the carrier whose radius is fixed.

In this way, since the rotational speed of elementary particles must be the speed of light, the minimum radius of elementary particles is expressed as $r_{0}=\frac{\Delta r}{2}=\frac{\mathrm{k}_{\mathrm{sp}} \times 2 \pi s}{M}=\frac{\lambda}{2 \pi} \times s$.

\subsection{Shape and Internal Structure of Elementary Particles}

From equations (4.35) and (5.8), we discuss the shape and internal structure of elementary particles.

First of all, we discuss the shape of elementary particles. From equation (5.8), the Compton wavelength $\lambda$ is expressed as

$$
\lambda=\frac{2 \pi r_{0}}{s} .
$$

From equation (5.10), when the spin quantum number is 1 , the Compton wavelength of elementary particles corresponds to the circumference at the minimum radius of elementary particles. Equation (5.10) shows that the shape of elementary particles is the circular particle with the length of $\lambda=\frac{2 \pi r_{0}}{s}$.

First, equation (5.3) must hold. That is, the mass must be distributed on the radius

$r_{0}$. For this reason, in the calculation of the radius of elementary particles based on the 
spin angular momentum, we calculated assuming that the mass point rotates at the speed of light on the circumference of radius $r_{0}$. However, not the elementary particles of the mass point rotate on the radius of $\frac{\Delta r}{2}$, but the elementary particles with the radius of $\frac{\Delta r}{2}$ rotate. Therefore, the shape of elementary particles is not a mass point, but a shape in which the mass is distributed on the radius $r_{0}$.

Second, from equation (5.10), the Compton wavelength of elementary particles is $\lambda=\frac{2 \pi r_{0}}{s}$, which is the length of the circumference at the minimum radius of elementary particles. This means that the wave of elementary particles is moving at the speed of light on the circumference.

Third, from quantum mechanics, an elementary particle is both a wave and a particle, and the wave energy is $E=\mathrm{h} f=\frac{\mathrm{hc}}{\lambda}$.

Fourth, from the theory of relativity and $E=\mathrm{h} f$, energy is equivalent to mass and is expressed as $E=m \mathrm{c}^{2}=\mathrm{h} f$.

From these, we conclude that the mass of elementary particles is distributed in the space where the energy of one wavelength of a wave is distributed. That is, the shape of elementary particles is the circular particle with the radius of $r_{0}=\frac{\Delta r}{2}$ and the length of $\lambda=\frac{2 \pi r_{0}}{s}$. An elementary particle rotates as a circular particle, and at the same time, move on the circumference as a wave.

From equation (4.35), we discuss the internal structure of elementary particles. First, equation (4.35) shows that the mass of all matter is made of spacetime particles. Spacetime is also made of spacetime particles. Therefore, all the masses composing the universe are made of spacetime particles. Second, the space of $\frac{\Delta r}{2}$ or less where gravitons are not distributed is spherical. An elementary particle exists in that space. Spacetime particles composing the mass of an elementary particle exist in the elementary particle. Third, the shape of spacetime particles is determined as a surface where the force from the center is constant. Fourth, if a force works inside an elementary particle, the surface where the force is constant is the spherical surface. Fifth, the shape of spacetime particles is the same spherical surface particles whether they are in spacetime or in elementary particles. Sixth, the spacetime structure composed of spacetime particles is the quantized 3-dimensional sphere of Poincaré conjecture. Therefore, we conclude that like the spacetime structure, the internal structure of elementary particles is the quantized 3-dimensional sphere of Poincaré conjecture.

We show the equation of the spacetime particle distribution in elementary particles.

The equation has two conditions that must be met. First, the radius of elementary particles is $\frac{\Delta r}{2}$. That is, the radius of the outermost spacetime particle in the elementary particle is $\frac{\Delta r}{2}$. Second, the internal structure of elementary particles is represented as the quantized 3-dimensional sphere of Poincaré conjecture.

From the two conditions, we show the spacetime particle distribution in elementary particles. From equations (3.16) and (5.5), the mass $m_{s p}$ of spacetime particles is expressed as 


$$
m_{s p}=T_{s p} \times \mathrm{m}_{\mathrm{sp} 0}=4 \pi s \times \mathrm{m}_{\mathrm{sp} 0} .
$$

The maximum number $n_{\max }$ of spacetime particles within an elementary particle is expressed as

$$
n_{\max }=\frac{m}{m_{s p}} .
$$

The radius $r_{0}$ of the elementary particle is expressed as

$$
r_{0}=n_{\max } \times \Delta r_{i n},
$$

where $\Delta r_{i n}$ is the distance between spacetime particles in the elementary particle and is expressed as

$$
\Delta r_{i n}=\frac{r_{0}}{n_{\max }} .
$$

The radius $r$ of spacetime particles in the elementary particle is expressed as

$$
r=n \Delta r_{i n}\left(\Delta r_{i n} \leqq r \leqq r_{0}\right),
$$

where $n\left(1 \leqq n \leqq n_{\max }\right)$ is the spacetime particle number in the elementary particle. The spacetime particle number density $\frac{\mathrm{d} n}{\mathrm{~d} V}$ in the elementary particle is expressed as

$$
\frac{\mathrm{d} n}{\mathrm{~d} V}=\frac{1}{4 \pi r^{2} \times \Delta r_{i n}} .
$$

From these, each spacetime particle in the elementary particle is a harmonic oscillator and thermally oscillates with $r=n \times \Delta r_{i n}$ as a fixed point, and its radius $r$ thermally oscillates in the following range:

$$
n \Delta r_{i n}-\frac{\Delta r_{i n}}{2}<r \leqq n \Delta r_{i n}+\frac{\Delta r_{i n}}{2}(n=1,2,3 \ldots) .
$$

The mass density of the elementary particle is expressed as

$$
m_{s p} \frac{\mathrm{d} n}{\mathrm{~d} V}=\frac{m_{s p}}{4 \pi r^{2} \times \Delta r_{i n}} .
$$

From equation (5.18), the mass density decreases in inverse proportion to the square of the radius from the center, the mass density at the center is the highest, and the mass density is the lowest at the radius $r_{0}$ of the elementary particle.

We discuss graviton coordinates and real coordinates in elementary particles. The circular particles derived from equation (5.8) are different from the quantized 3-dimensional sphere 
derived from equation (4.35). Both are inconsistent because they are the same spacetime particle distribution in an elementary particle. The difference in this distribution is the difference between graviton coordinates and real coordinates.

Graviton coordinates are the coordinates of an object related to the graviton, and real coordinates are the coordinates where the object is actually located. For example, even if an object is located at a certain point in real coordinates, the object is distributed on the spherical surface in graviton coordinates. In this way, even with the coordinates of the same object, the coordinates are different between graviton coordinates and real coordinates.

Since in graviton coordinates there are no gravitons in the space of $\frac{\Delta r}{2}$ or less, elementary particles rotate at the speed of light and the radius of elementary particles must be $\frac{\Delta r}{2}$. Therefore, elementary particles in graviton coordinates are circular particles with the radius $\frac{\Delta r}{2}$ and the length $\frac{2 \pi r_{0}}{s}$, no matter how they are actually distributed.

On the other hand, in real coordinates, coordinates can be freely taken in the space of $\frac{\Delta r}{2}$ or less. Therefore, in real coordinates, the spacetime particle distribution in elementary particles becomes the quantized 3-dimensional sphere.

We summarize the difference between graviton coordinates and real coordinates.

- Graviton coordinates:

If $r \leqq \frac{\Delta r}{2}, r=\frac{\Delta r}{2}$, and gravity $F$ is $F=0$.

If $N \Delta r-\frac{\Delta r}{2}<r \leqq N \Delta r+\frac{\Delta r}{2}, r=N \Delta r$, and gravity $F$ is $F \neq 0$.

If $r>\left(N+\frac{1}{2}\right) \Delta r, r=\left(N+\frac{1}{2}\right) \Delta r$, and gravity $F$ is $F=0$.

An elementary particle is a circular particle with the mass $m$, the radius $\frac{\Delta r}{2}$ and the length $\frac{2 \pi r_{0}}{s}$.

- Real coordinates in $r \leqq \frac{\Delta r}{2}$ :

$r=n \times \Delta r_{i n}\left(\Delta r_{i n} \leqq r \leqq r_{0}\right)$.

Spacetime particles in an elementary particle are spherical surface particles with the mass $m_{s p}$, the radius $r=n \times \Delta r_{i n}$ and the volume $4 \pi r^{2} \times \Delta r_{i n}$.

\subsection{Elementary Particle Radius and Observation Problem}

We discuss the difference between the derived elementary particle radius and the observed elementary particle radius. Then we discuss the properties within elementary particles.

Observation suggests that the upper limit of an electron's radius is $10^{-22}$ meters [15]. The radius of an electron based on $r_{0}=\frac{\Delta r}{2}=\frac{\lambda}{2 \pi} \times s$ is $1.9307963398 \times 10^{-13}$ meters. Therefore, the theory of elementary particle radius is inconsistent with the observation. This is a problem and we discuss this problem. 
Assumption 5.1: An elementary particle is composed of spacetime particles with energy and charged particles with charges, and an elementary particle has the radius of spacetime particles that are difficult to observe and the radius of charged particles that are easy to observe.

First, dark matter, which is spacetime particles, is extremely difficult to observe because it interacts only with gravity. Therefore, spacetime particles have mass but no charge, and it is extremely difficult to observe spacetime particles.

Second, since spacetime particles have no charge, another particle must have charges within an elementary particle. Therefore, in an elementary particle, apart from spacetime particles with mass or energy, there are charged particles with charges. For example, an electron and a positron annihilate and turn into light energy. Conversely, light energy produces an electron-positron pair. This fact suggests that electrons and positrons are made from charges and photon energy.

Third, the gravitational interaction is so weak that it is extremely difficult to observe spacetime particles. Conversely, the interaction by charges is so strong that it is easy to observe charged particles.

Fourth, in the structure of spacetime, there is ordinary matter that can be observed in the center, and spacetime particles that are difficult to observe are distributed around the matter. Similarly, in the internal structure of an elementary particle, there are charged particles that can be observed in the center, and spacetime particles that are difficult to observe are distributed around charged particles. Therefore, an elementary particle has the spacetime particle radius by spacetime particles and the charge radius by charged particles.

From these, we conclude assumption 5.1. From assumption 5.1, the observable elementary particle radius is the charge radius, but the actual elementary particle radius is the spacetime particle radius of $\frac{\Delta r}{2}$ that is difficult to observe. This can explain the theory and observation of an elementary particle radius without contradiction.

We show the concrete spacetime particle radius and charge radius. From equation (5.8), the spacetime particle radius $r_{0}$ is $r_{0}=\frac{\Delta r}{2}=\frac{\lambda}{2 \pi} \times s$. Like the spacetime particle radius $r_{0}$, the charge radius $r_{0 c}$ is $\frac{\Delta r_{i n}}{2}$. In the space of $r_{0 c}$ or less, spacetime particles in an elementary particle are not distributed. Therefore, from equation (5.14), the charge radius $r_{0 c}$ is expressed as

$$
r_{0 c}=\frac{\Delta r_{i n}}{2}=\frac{r_{0}}{2 n_{\max }}=\frac{\lambda}{4 \pi n_{\max }} \times s .
$$

As an example, we calculate the spacetime particle radius and charge radius of an electron. From equation (5.7), the spacetime particle radius $r_{0}$ of an electron is $r_{0}=1.9307963398 \times$ $10^{-13}$ meters. From equation (5.19), the charge radius $r_{0 c}$ of an electron is expressed as

$$
r_{0 c}=6.98 \times 10^{-45} \text { meters } .
$$


Since the mass of an electron is small, the radius of the electron is relatively large. Despite that, the charge radius of the electron is only $6.98 \times 10^{-45}$ meters. Therefore, we conclude that the charge radii of all elementary particles can be observed almost as point particles.

Since the observable elementary particle radius is the charge radius $r_{0 c}$, we observe elementary particles as if they were point particles. However, the actual elementary particle radius is the spacetime particle radius $r_{0}=\frac{\Delta r}{2}=\frac{\lambda}{2 \pi} \times s$.

We apply the minimum space properties to spacetime particles in an elementary particle, and we discuss the properties within elementary particles.

First, no matter how charged particles are distributed in the space of $\frac{\Delta r_{i n}}{2}$ or less, the radius of charged particles is $\frac{\Delta r_{i n}}{2}$.

Second, like the graviton, in the case of the things related to the charge, the entire charged particle of $\frac{\Delta r_{i n}}{2}$ or less is affected as one minimum space. That is, in that case, they can be regarded as if they were rigid bodies or point particles.

Third, spacetime particles and charged particles within the radius of $\frac{\Delta r}{2}$ or less are in the minimum space that cannot be divided in graviton coordinates. Therefore, in the case of the things related to gravitons, spacetime particles and charged particles cannot be regarded as separated. That is, in graviton coordinates, spacetime particles and charged particles appear to be one indivisible elementary particle.

Fourth, in the space of $r_{0}=\frac{\Delta r}{2}$ or less, a gravitational field becomes zero. Similarly, in the space of $r_{0 c}=\frac{\Delta r_{i n}}{2}$ or less, a charge field becomes zero. This is because all fields work only in the presence of spacetime particles, which are spacetime. For example, in the space where there is no spacetime before the birth of the universe, no force works. It is the same as this.

Fifth, in the space of $r_{0 c}=\frac{\Delta r_{i n}}{2}$ or less where charged particles exist, a charge field is zero, so the electrostatic energy and so on become zero.

Sixth, since the electrostatic energy and so on are zero, the mass of elementary particles is determined only by the mass or energy of spacetime particles.

In this way, we can explain the properties within elementary particles.

\subsection{Internal Structure of Protons and Radii of Protons and Deuterons}

Assuming the internal structure of protons, we discuss the radii of protons and deuterons based on the charge radius and the spacetime particle radius. Assumption 5.1 can explain observations and theories on an elementary particle radius without contradiction. If assumption 5.1 is correct, it must also be able to explain the radii of protons and deuterons that are composite particles. Therefore, we discuss the radii of protons and deuterons from assumption 5.1 .

Assumption 5.2: Inside a proton, there are only four elementary particles of two up quarks, one down quark, and one gluon. However, inside each elementary particle, there are 
spacetime particles and charged particles that compose the elementary particle.

Assumption 5.3: Regardless of the mass when decomposed into individual elementary particles, each elementary particle inside a proton has the quartered mass of a proton. For this reason, the mass $m$ of each elementary particle is expressed as

$$
m=m_{u}=m_{d}=m_{l}=\frac{M}{4}
$$

where $M$ is the mass of a proton, $m_{u}$ is the mass of an up quark, $m_{d}$ is the mass of a down quark, and $m_{l}$ is the mass of a gluon.

We express the radius of elementary particles within a proton by the minimum radius of the proton. The spins of protons and up quarks and down quarks are $1 / 2$, and the spin of gluons is 1 . The minimum radius $r_{\text {min }}$ of a proton is an elementary particle radius $r_{0}=\frac{\Delta r}{2}$. This radius is the radius when a proton is considered as an elementary particle. From equation (5.6) and $s=1 / 2$, the minimum radius $r_{m i n}$ of a proton is expressed as

$$
r_{\min }=\frac{\mathrm{k}_{\mathrm{sp}} \times \pi}{M}
$$

Using the radius $r_{m i n}$, the elementary particle radius $r_{u}$ of an up quark is expressed as

$$
r_{u}=4 r_{\min },
$$

the elementary particle radius $r_{d}$ of a down quark is expressed as

$$
r_{d}=4 r_{\min },
$$

the elementary particle radius $r_{g}$ of a gluon is expressed as

$$
r_{g}=8 r_{\min },
$$

and the elementary particle radius $r_{Q}$ of quarks within a proton is expressed as

$$
r_{Q}=r_{u}=r_{d}=4 r_{\min } .
$$

Assumption 5.4: As shown in figure 3, a gluon is in the center, and two up quarks and one down quark are distributed farthest from each other on the spherical surface of the gluon. Therefore, when we connect two up quarks and one down quark with a line, it has the shape of a regular triangle. 


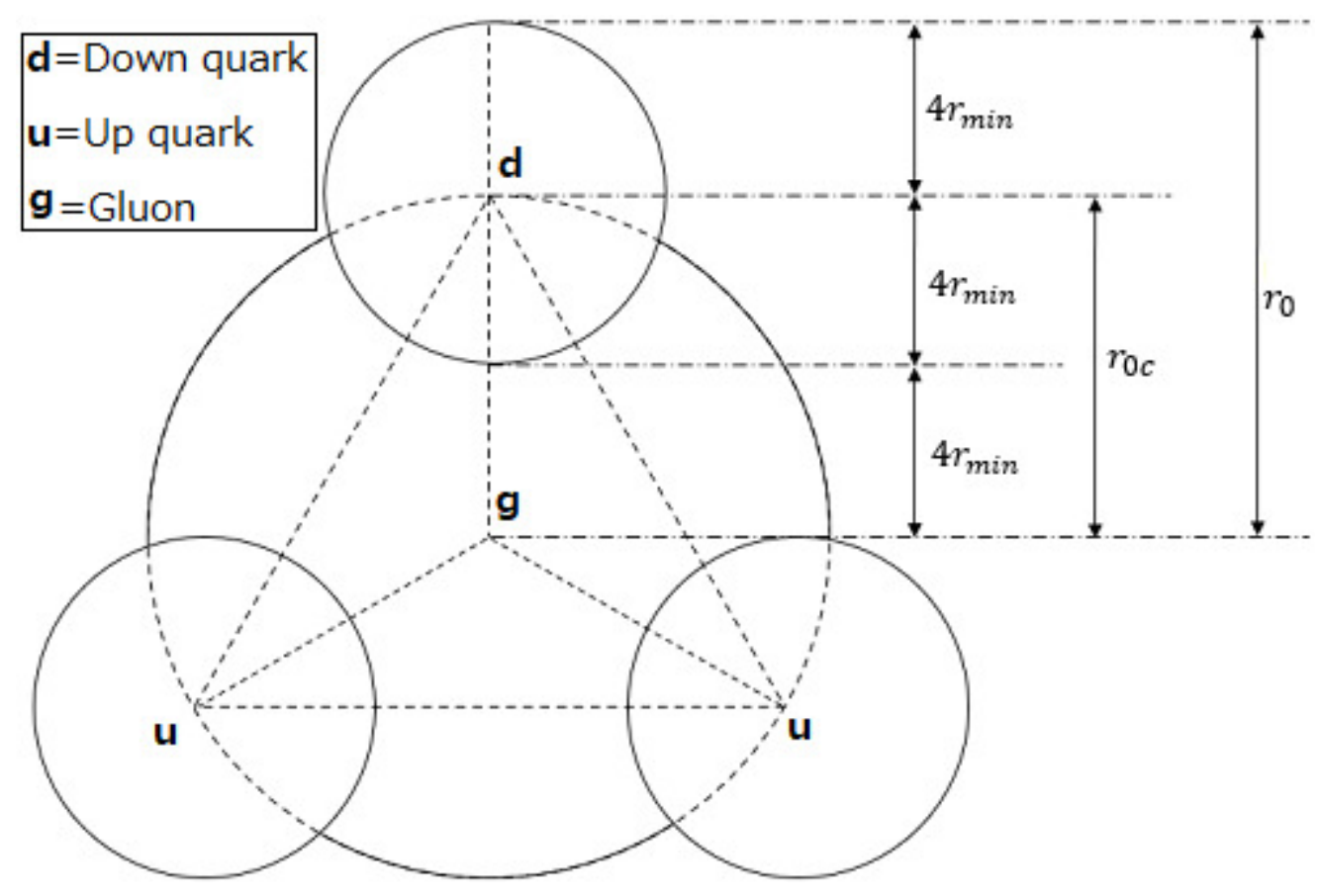

Figure 3: The internal structure of protons. Inside a proton, there are only four elementary particles of two up quarks, one down quark, and one gluon. Gluons are spherical particles with a radius $8 r_{\text {min }}$, and up quarks and down quarks are spherical particles with a radius $4 r_{\min }$. A gluon is in the center, and two up quarks and one down quark are distributed farthest from each other on the spherical surface of the gluon. The radius $r_{0 c}$ is the charge radius of protons, the radius $r_{0}$ is the spacetime particle radius of protons, and the radius $r_{\text {min }}$ is the minimum radius $\frac{\Delta r}{2}=\frac{\lambda}{2 \pi} s$ based on the mass of a proton.

From the simplicity principle, assumptions 5.2 to 5.4 are assumptions to explain the radius more simply.

We compare the theoretical and measured values of the proton charge radius. From assumption 5.4 and figure 3 , the charge radius $r_{0 c}$ of a proton is expressed as

$$
r_{0 c}=r_{g}=8 r_{\min }
$$

and the spacetime particle radius $r_{0}$ of a proton is expressed as

$$
r_{0}=r_{g}+r_{Q}=12 r_{\min } .
$$

In this way, in the case of composite particle protons, from its internal structure, the charge radius does not become a point particle, and the spacetime particle radius does not become the minimum radius based on the mass of the composite particle. 
From the proton mass $M=1.67262192369 \times 10^{-27} \mathrm{~kg}$ (2018 CODATA value), the minimum radius $r_{\min }$ is expressed as

$$
r_{\text {min }}=1.05154455168 \times 10^{-16} \text { meters . }
$$

Therefore, the theoretical value $r_{0 c}$ of the proton charge radius is expressed as

$$
r_{0 c}=8.41235641342 \times 10^{-16} \text { meters } .
$$

The measured value $r_{0 c \mu}$ of the proton charge radius is expressed as

$$
r_{0 c \mu}=8.414 \times 10^{-16} \text { meters (2018 CODATA recommended values) } .
$$

The relative error is expressed as follows.

$$
\text { Relative error between } r_{0 c \mu} \text { and } r_{0 c}=0.01954 \% \text {. }
$$

The theoretical value $r_{0 c}$ of the proton charge radius almost agrees with the measured value $r_{0 c \mu}$. Therefore, the theoretical value $r_{0 c}$ of the proton charge radius is valid.

We compare the theoretical and measured values of the deuteron charge radius. A deuteron is a particle composed of one proton and one neutron. Since the mass of a neutron is almost the same as that of a proton and its internal structure is considered to be the same as that of a proton, the radius of a neutron is almost the same as the radius of a proton. Therefore, we consider that the radius of a neutron is equal to the radius of a proton. In the case of deuterons, we assume that one proton and one neutron are in contact with each other at the spacetime particle radius as shown in figure 4. 


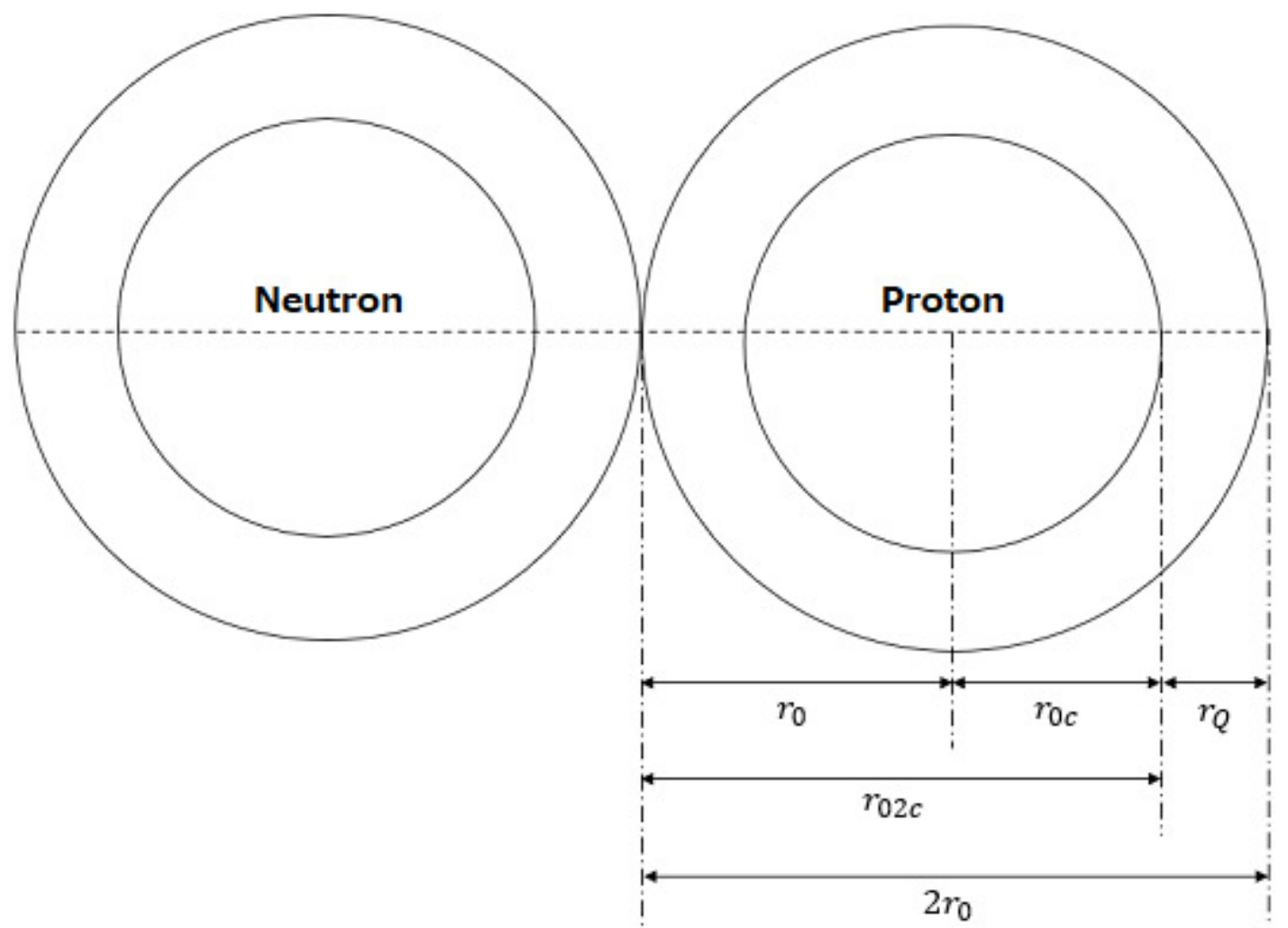

Figure 4: The simplified internal structure of deuterons. The radii of protons and neutrons are almost the same, and their charge radii $r_{0 c}$ and spacetime particle radii $r_{0}$ are drawn. One proton and one neutron are in contact with each other at the spacetime particle radius $r_{0}$. The radius $r_{Q}$ is the spacetime particle radius of quarks, the radius $r_{02 c}$ is the charge radius of deuterons, and the radius $2 r_{0}$ is the spacetime particle radius of deuterons.

From figure 4 , the charge radius $r_{02 c}$ of a deuteron is expressed as

$$
r_{02 c}=r_{0}+r_{0 c} .
$$

Therefore, from the charge radius $r_{02 c}$ of a deuteron, we can verify the spacetime particle radius $r_{0}$ of a proton. The theoretical value $r_{02 c}$ of the deuteron charge radius is expressed as

$$
r_{02 c}=2.10308910336 \times 10^{-15} \text { meters } .
$$

The measured value $r_{02 c \mu}$ of the deuteron charge radius is expressed as

$$
r_{02 c \mu}=2.12799 \times 10^{-15} \text { meters (2018 CODATA recommended values) } .
$$


The relative error is expressed as follows.

$$
\text { Relative error between } r_{02 c \mu} \text { and } r_{02 c}=1.18402 \% \text {. }
$$

The theoretical value $r_{02 c}$ of the deuteron charge radius agrees with the measured value $r_{02 c \mu}$ with an error of approximately $1 \%$. Therefore, the calculation of the deuteron charge radius is valid. The spacetime particle radius of a deuteron is twice the spacetime particle radius of a proton from figure 4 .

We compare the theoretical and measured values of the spacetime particle radius of a proton. From equation (5.33), the equation of the measured value $r_{0 \mu}$ of spacetime particle radius of a proton is expressed as

$$
r_{0 \mu}=r_{02 c \mu}-r_{0 c \mu} .
$$

From equation (5.28), the theoretical value $r_{0}$ of spacetime particle radius of a proton is expressed as

$$
r_{0}=1.26185346201 \times 10^{-15} \text { meters . }
$$

From equation (5.37), the measured value $r_{0 \mu}$ of spacetime particle radius of a proton is expressed as

$$
r_{0 \mu}=1.287 \times 10^{-15} \text { meters } .
$$

The relative error is expressed as follows.

$$
\text { Relative error between } r_{0 \mu} \text { and } r_{0}=1.960 \% \text {. }
$$

Therefore, the theoretical value $r_{0}$ of the spacetime particle radius of a proton agrees with the measured value $r_{0 \mu}$ with an error of approximately $2 \%$.

We compare the theoretical and measured values of the spacetime particle radius of quarks within a proton. From figure 4, the equation of the measured value $r_{Q \mu}$ of the spacetime particle radius of quarks is expressed as

$$
r_{Q \mu}=r_{0 \mu}-r_{0 c \mu} .
$$

From equation (5.26), the theoretical value $r_{Q}$ of spacetime particle radius of quarks is expressed as

$$
r_{Q}=4.20617820671 \times 10^{-16} \text { meters . }
$$

From equation (5.41), the measured value $r_{Q \mu}$ of spacetime particle radius of quarks is expressed as 


$$
r_{Q \mu}=4.452 \times 10^{-16} \text { meters } .
$$

The relative error is expressed as follows.

$$
\text { Relative error between } r_{Q \mu} \text { and } r_{Q}=5.842 \% \text {. }
$$

Therefore, the theoretical value $r_{Q}$ of the spacetime particle radius of quarks agrees with the measured value $r_{Q \mu}$ with an error of approximately $6 \%$.

Although assumptions have been added and there is an error, the charge radius and spacetime particle radius of assumption 5.1 can explain the observation of the charge radii of protons and deuterons. Importantly, the presence of the directly unobservable spacetime particle radius could be confirmed from the charge radius. Therefore, assumption 5.1 is valid.

\subsection{Nuclear Radius}

We discuss the nuclear radius. An atomic nucleus is composed of protons and neutrons, and the nuclear radius $r_{0 A c}$ is expressed as

$$
r_{0 A c}=r_{0} \times A^{\frac{1}{3}},
$$

where $A$ is the mass number that is the sum of the number of protons and neutrons, $r_{0}$ is $1.25 \times 10^{-15}$ meters. Note that the nuclear radius of equation (5.45) is the charge radius, not the spacetime particle radius.

From equation (5.38), the theoretical value of the spacetime particle radius of a proton is $1.26185346201 \times 10^{-15}$ meters. The spacetime particle radius of neutrons is almost equal to that of protons. Therefore, the value of $r_{0}=1.25 \times 10^{-15}$ almost agrees with the spacetime particle radius of protons and neutrons. That is, this shows that the value of $r_{0}$ is the spacetime particle radius of protons and neutrons.

We derive the nuclear radius when the mass number $A$ is large. The radius $r_{0}$ is the spacetime particle radius of one proton or neutron, and the sphere volume $V_{0}$ with the radius $r_{0}$ is expressed as

$$
V_{0}=\frac{4 \pi r_{0}^{3}}{3}
$$

The sphere volume $V_{c}$ with the nuclear radius $r_{0 A}$ is expressed as

$$
V_{c}=\frac{4 \pi r_{0 A}^{3}}{3}
$$

We assume that protons and neutrons are in contact with each other at the spacetime particle radius and form a spherical structure as an entire. Then, the mass number $A$ is expressed as the number of the volume $V_{0}$ included in the volume of $V_{c}$, so $A$ is expressed as 


$$
A=\frac{V_{c}}{V_{0}}=\frac{r_{0 A}^{3}}{r_{0}^{3}} .
$$

From equation (5.48), the nuclear radius $r_{0 A}$ is expressed as

$$
r_{0 A}=r_{0} \times A^{\frac{1}{3}}
$$

The nuclear radius $r_{0 A}$ of equation (5.49) agrees with the nuclear radius $r_{0 A c}$ of equation (5.45). Therefore, we conclude that $r_{0}$ in equation (5.45) is the spacetime particle radius of one proton or neutron.

Note that the nuclear radius $r_{0 A c}$ of equation (5.45) is the charge radius of an atomic nucleus, and the nuclear radius $r_{0 A}$ of equation (5.49) is the spacetime particle radius of that. When the mass number is large, the charge radius $r_{0 A c}$ of the atomic nucleus almost agrees with the spacetime particle radius $r_{0 A}$ of that, but the spacetime particle radius $r_{0 A}$ is slightly larger than the charge radius $r_{0 A c}$. Therefore, $r_{0}=1.26185346201 \times 10^{-15}$ of equation (5.49) is slightly larger than $r_{0}=1.25 \times 10^{-15}$ of equation $(5.45)$.

\section{Expansion Speed and Expansion Acceleration of Spacetime}

In this section, we discuss the expansion speed and expansion acceleration of spacetime from the equation of state of spacetime particles. From the equation of state of spacetime particles and some assumptions and premises and conclusions, we derive equations related to the expansion of spacetime and show what is happening in the expansion of spacetime. Note that in this paper, an assumption is a conclusion that we consider to be correct without clear evidence, and a premise is a condition setting. That is, an assumption needs to be proven, but a premise is a condition, state, or setting that can be freely determined.

\subsection{Assumptions and Premises and Conclusions in the Expansion of Spacetime}

To explain the expansion of spacetime with the equation of state of spacetime particles, we make the following assumptions, premises, and conclusions.

Conclusion 6.1: The expansion source of spacetime is at the center of individual matter with active gravitational mass. First, from the equation of state of spacetime particles, the spacetime radius expands from the center of matter. Second, since spacetime particles around matter move away at the speed of light, it is necessary to always produce new spacetime particles at the center of the matter. Otherwise, spacetime particles will not always exist around matter, and gravity will not be carried by spacetime particles. Therefore, we conclude that the expansion source of spacetime is at the center of individual matter. 
Premise 6.2: All expansion sources expand space in the same way, and the expansion of the entire outer space can be considered based on any one expansion source. If the expansion sources of individual matter expand space in the same way, all space appears to expand in the same way from any expansion source. For this reason, when viewed from any one expansion source, it appears as if the entire outer space is expanding by the expansion of that expansion source. Therefore, by considering the expansion by any one expansion source, we can consider the expansion of the entire outer space. With such a simple condition, we consider the expansion of the universe.

Conclusion 6.3: When the spacetime particle number $N$ is the maximum number $N_{\max }$ at time $t$, the spacetime radius $r_{\max }\left(N=N_{\max }\right)$ of equation (2.18) is the maximum spacetime radius for one expansion source and is expressed as

$$
r_{\max }=\mathrm{k}_{\mathrm{sp}} T_{s p} \times \frac{N_{\max }}{M}
$$

where $M$ and $N_{\max }$ are the mass and maximum spacetime particle number of any one expansion source at time $t$. In this section, the spacetime radius refers to the maximum spacetime radius of equation (6.1).

Premise 6.4: The time $t=0$ is the time when the deceleration expansion changes to the acceleration expansion, $t=t_{1}$ is the current time, and $t=t_{0}$ is the universe's birth time. Since it is easy to calculate, the time at which the deceleration expansion changes to the acceleration expansion is set to $t=0$ in this paper.

Assumption 6.5: In some way, the mass $M$ of ordinary matter and the spacetime particle number $N$ change and spacetime expands, and the mass $M$ of ordinary matter is expressed as

$$
M=\beta_{2} t^{2}+\beta_{1} t+M_{0}
$$

and the spacetime particle number $N$ is expressed as

$$
N=\alpha_{1} t+N_{0}
$$

where $\beta_{2}, \beta_{1}$ and $\alpha_{1}$ are a constant, $M_{0}$ is the mass of ordinary matter at $t=0$, and $N_{0}$ is the spacetime particle number at $t=0$.

Equations (6.2) and (6.3) are derived as simple equations that fit the observations. We don't consider how to change them, only the expansion that is the result of the change. From $r=\mathrm{k}_{\mathrm{sp}} T_{s p} \times \frac{N}{M}$ of equation (2.18), we can explain that the spacetime radius expands by three factors: the increase in the spacetime particle number $N$, the decrease in the mass $M$ of ordinary matter, and the increase in the dimensionless temperature $T_{s p}$ of spacetime particles. However, since $T_{s p}$ is expressed as $T_{s p}=4 \pi s$ of equation (5.5) and $s$ is the spin quantum number of ordinary matter, $T_{s p}$ does not change. Therefore, the spacetime radius expands by the spacetime particle number $N$ and the mass $M$ of ordinary matter. 
Note that even if the total mass of the universe is always constant, the mass $M$ of ordinary matter can change. From the mass definition of assumptions 2.9 and 2.10, the mass $M$ of the equation of state of spacetime particles is active gravitational mass. For this reason, the mass $M$ does not include the mass of particles without active gravitational mass, such as photons and gravitons. Therefore, when matter changes from particles with active gravitational mass to particles without that, or from particles without active gravitational mass to particles with that, the mass $M$ changes.

Assumption 6.6: There is a radius $r$ from the matter of the expansion source to another matter within the spacetime radius $r_{\max }$. The relationship between individual elements is expressed as

$$
\begin{gathered}
\frac{v}{v_{\max }}=\frac{r}{r_{\max }}, \\
\frac{a}{a_{\max }}=\frac{r}{r_{\max }},
\end{gathered}
$$

where $r_{\text {max }}$ is the maximum spacetime radius, $r\left(0 \leqq r \leqq r_{\text {max }}\right)$ is the radius from the matter of the expansion source to another matter, $v_{\max }$ is the expansion speed of the maximum spacetime radius $r_{\max }$, and $v$ is the expansion speed of the radius $r$.

From conclusion 6.1, the expansion source is at the center of matter, and the radius $r$ is the radius from the matter of the expansion source to another matter. We call this radius $r$ the inter-matter radius. The inter-matter radius $r$ represents the radius within the spacetime radius $r_{\max }$, but the physical meaning is different from the spacetime radius as follows.

The spacetime radius $r_{\max }$ is the radius from the center of the matter of the expansion source to the spacetime particle without active gravitational mass.

The inter-matter radius $r$ is the radius from the center of the matter of the expansion source to another matter with active gravitational mass.

In this way, the starting point of the radius is the same, but the ending point of the radius is different. Since the spacetime radius $r_{\max }$ is the radius to the spacetime particle without active gravitational mass, the expansion speed $v_{\max }$ of the spacetime radius $r_{\max }$ is usually the speed of light. Since the inter-matter radius $r$ is the radius to another matter with active gravitational mass, the expansion speed $v$ of the inter-matter radius $r$ is usually less than the speed of light. Therefore, the spacetime radius $r_{\max }$ and the inter-matter radius $r$ do not always change in exactly the same way because they have different physical meanings. As their relationship, we assume equations (6.4) and (6.5). Equations (6.4) and (6.5) mean that the expansion of the inter-matter radius $r$ is caused by the expansion of the spacetime radius $r_{\max }$. Assuming equations (6.4) and (6.5), the expansion speed $v$ and the expansion acceleration $a$ are expressed as a function of the inter-matter radius $r$.

Note that the expansion we are observing is not the expansion of the spacetime radius, but the expansion of the inter-matter radius. This is because the expansion speed we are 
observing is not the speed at which spacetime particles move away, but the speed at which matter moves away. However, since the expansion of the inter-matter radius is caused by the expansion of the spacetime radius, it is not a mistake to refer to the expansion of the inter-matter radius as the expansion of spacetime.

Based on the above assumptions and premises and conclusions, we describe the expansion of spacetime from the equation of state of spacetime particles.

\subsection{Derivation of Expansion Speed, Expansion Acceleration, Hubble Parameter and Deceleration Parameter}

We derive the expansion speed, the expansion acceleration, the Hubble parameter and the deceleration parameter.

First of all, we show the mass change rate of ordinary matter, spacetime particle number change rate and spacetime expansion rate from the reference time $t=0$. When we rearrange equations (6.2) and (6.3), they are expressed as

$$
\begin{gathered}
\frac{M}{M_{0}}=\frac{\beta_{2}}{M_{0}} t^{2}+\frac{\beta_{1}}{M_{0}} t+1, \\
\frac{N}{N_{0}}=\frac{\alpha_{1}}{N_{0}} t+1 .
\end{gathered}
$$

We set the constant $a_{1}, b_{1}$ and $b_{2}$ as

$$
\begin{aligned}
& a_{1}=\frac{\alpha_{1}}{N_{0}}, \\
& b_{1}=\frac{\beta_{1}}{M_{0}}, \\
& b_{2}=\frac{\beta_{2}}{M_{0}} .
\end{aligned}
$$

From equations (6.8) and (6.9) and (6.10), equations (6.2) and (6.3) are expressed as

$$
\begin{gathered}
M=M_{0}\left(b_{2} t^{2}+b_{1} t+1\right), \\
N=N_{0}\left(a_{1} t+1\right)
\end{gathered}
$$

and equations (6.6) and (6.7) are expressed as

$$
M_{R}=\frac{M}{M_{0}}=b_{2} t^{2}+b_{1} t+1
$$




$$
N_{R}=\frac{N}{N_{0}}=a_{1} t+1
$$

where $M_{R}$ is the mass change rate of ordinary matter from the reference time $t=0$, and $N_{R}$ is the spacetime particle number change rate from the reference time $t=0$. From equations (6.1) and (6.2) and (6.3), the spacetime radius $r_{\max }(t=0)$ at $t=0$ is expressed as

$$
r_{\text {max }}(t=0)=\mathrm{k}_{\mathrm{sp}} T_{s p} \times \frac{N_{0}}{M_{0}} .
$$

Therefore, the spacetime expansion rate $E_{R}$ from the reference time $t=0$ is expressed as

$$
E_{R}=\frac{r_{\max }(t)}{r_{\max }(t=0)}=\frac{N_{R}}{M_{R}}=\frac{a_{1} t+1}{b_{2} t^{2}+b_{1} t+1} .
$$

From equations (6.11) and (6.12), the spacetime radius $r_{\max }$ is expressed as

$$
r_{\text {max }}=\mathrm{k}_{\mathrm{sp}} T_{s p} \times \frac{N_{0}}{M_{0}} \times \frac{a_{1} t+1}{b_{2} t^{2}+b_{1} t+1} .
$$

From equations (6.15) and (6.16), the spacetime radius $r_{\max }$ is also expressed as

$$
r_{\text {max }}=r_{\text {max }}(t=0) \times \frac{a_{1} t+1}{b_{2} t^{2}+b_{1} t+1}=r_{\text {max }}(t=0) \times E_{R} .
$$

We derive the expansion speed of the spacetime radius $r_{\max }$. The expansion speed $v_{\max }$ of the spacetime radius $r_{\max }$ is expressed as

$$
v_{\max }=\frac{\mathrm{d} r_{\max }}{\mathrm{d} t} .
$$

From equations (6.18) and (6.19), the expansion speed $v_{\max }$ of the spacetime radius $r_{\max }$ is expressed as

$$
v_{\text {max }}=r_{\text {max }}(t=0) \times \frac{-a_{1} b_{2} t^{2}-2 b_{2} t+a_{1}-b_{1}}{\left(b_{2} t^{2}+b_{1} t+1\right)^{2}} .
$$

From $r_{\max }=r_{\max }(t=0) \times E_{R}$, the expansion speed $v_{\max }$ is also expressed as

$$
v_{\max }=r_{\max }(t=0) \times \frac{\mathrm{d} E_{R}}{\mathrm{~d} t} .
$$

From equations (6.20) and (6.21), $\frac{\mathrm{d} E_{R}}{\mathrm{~d} t}$ is expressed as

$$
\frac{\mathrm{d} E_{R}}{\mathrm{~d} t}=\frac{-a_{1} b_{2} t^{2}-2 b_{2} t+a_{1}-b_{1}}{\left(b_{2} t^{2}+b_{1} t+1\right)^{2}} .
$$


We derive the expansion acceleration of the spacetime radius $r_{\max }$. Since we are considering expansion only in the radial direction, there is no rotation component, and the time derivative $\frac{\mathrm{d} \theta}{\mathrm{d} t}$ to the angle $\theta$ of polar coordinates is 0 . Therefore, the expansion acceleration $a_{\max }$ of the spacetime radius $r_{\max }$ is expressed as

$$
a_{\max }=\frac{\mathrm{d} v_{\max }}{\mathrm{d} t}=\frac{\mathrm{d}^{2} r_{\max }}{\mathrm{d} t^{2}}
$$

From equations (6.20) and (6.23), the expansion acceleration $a_{\max }$ of the spacetime radius $r_{\text {max }}$ is expressed as

$$
a_{\text {max }}=2 r_{\text {max }}(t=0) \times \frac{a_{1} b_{2}^{2} t^{3}+3 b_{2}^{2} t^{2}-3 a_{1} b_{2} t+3 b_{1} b_{2} t-b_{2}-a_{1} b_{1}+b_{1}^{2}}{\left(b_{2} t^{2}+b_{1} t+1\right)^{3}} .
$$

From equation (6.21), the expansion acceleration $a_{\max }$ is also expressed as

$$
a_{\max }=r_{\max }(t=0) \times \frac{\mathrm{d}^{2} E_{R}}{\mathrm{~d} t^{2}} .
$$

From equations (6.24) and (6.25), $\frac{\mathrm{d}^{2} E_{R}}{\mathrm{~d} t^{2}}$ is expressed as

$$
\frac{\mathrm{d}^{2} E_{R}}{\mathrm{~d} t^{2}}=2 \times \frac{a_{1} b_{2}^{2} t^{3}+3 b_{2}^{2} t^{2}-3 a_{1} b_{2} t+3 b_{1} b_{2} t-b_{2}-a_{1} b_{1}+b_{1}^{2}}{\left(b_{2} t^{2}+b_{1} t+1\right)^{3}} .
$$

We derive the Hubble parameter. When we rearrange equation (6.4), it is expressed as

$$
v=r \times \frac{v_{\max }}{r_{\max }} .
$$

From equation (6.27), the expansion speed $v$ increases with increasing distance $r$, which is consistent with the observation of expansion based on the Hubble constant. The Hubble constant $H$ is expressed as

$$
H=\frac{v}{r}
$$

From equations (6.27) and (6.28), the Hubble constant $H$ is expressed as

$$
H=\frac{v}{r}=\frac{v_{\max }}{r_{\max }} \text {. }
$$

In this paper, since the Hubble constant is not a constant but a function of time $t$, it is the Hubble parameter. From equations (6.18) and (6.20) and (6.27), the expansion speed $v$ is expressed as 


$$
v=r \times \frac{-a_{1} b_{2} t^{2}-2 b_{2} t+a_{1}-b_{1}}{\left(b_{2} t^{2}+b_{1} t+1\right)\left(a_{1} t+1\right)} .
$$

From equations (6.28) and (6.30), the Hubble parameter $H$ is expressed as

$$
H=\frac{-a_{1} b_{2} t^{2}-2 b_{2} t+a_{1}-b_{1}}{\left(b_{2} t^{2}+b_{1} t+1\right)\left(a_{1} t+1\right)} .
$$

From equations (6.18) and (6.21) and (6.27), the expansion speed $v$ is also expressed as

$$
v=r \times \frac{\left(\frac{\mathrm{d} E_{R}}{\mathrm{~d} t}\right)}{E_{R}} .
$$

From equations (6.28) and (6.32), the Hubble parameter $H$ is also expressed as

$$
H=\frac{\left(\frac{\mathrm{d} E_{R}}{\mathrm{~d} t}\right)}{E_{R}}
$$

From equations (6.31) and (6.33), $\frac{\left(\frac{\mathrm{d} E_{R}}{\mathrm{~d} t}\right)}{E_{R}}$ is expressed as

$$
\frac{\left(\frac{\mathrm{d} E_{R}}{\mathrm{~d} t}\right)}{E_{R}}=\frac{-a_{1} b_{2} t^{2}-2 b_{2} t+a_{1}-b_{1}}{\left(b_{2} t^{2}+b_{1} t+1\right)\left(a_{1} t+1\right)} .
$$

We derive the acceleration parameter. When we rearrange equation (6.5), it is expressed as

$$
a=r \times \frac{a_{\max }}{r_{\max }} .
$$

Similar to the Hubble parameter, we define the acceleration parameter $A_{P}$ as

$$
A_{P}=\frac{a}{r}
$$

From equations (6.35) and (6.36), the acceleration parameter $A_{P}$ is expressed as

$$
A_{P}=\frac{a}{r}=\frac{a_{\max }}{r_{\max }} .
$$

From equations (6.18) and (6.24) and (6.35), the expansion acceleration $a$ is expressed as

$$
a=r \times 2 \times \frac{a_{1} b_{2}^{2} t^{3}+3 b_{2}^{2} t^{2}-3 a_{1} b_{2} t+3 b_{1} b_{2} t-b_{2}-a_{1} b_{1}+b_{1}^{2}}{\left(b_{2} t^{2}+b_{1} t+1\right)^{2}\left(a_{1} t+1\right)} .
$$

From equations (6.36) and (6.38), the acceleration parameter $A_{P}$ is expressed as 


$$
A_{P}=2 \times \frac{a_{1} b_{2}^{2} t^{3}+3 b_{2}^{2} t^{2}-3 a_{1} b_{2} t+3 b_{1} b_{2} t-b_{2}-a_{1} b_{1}+b_{1}^{2}}{\left(b_{2} t^{2}+b_{1} t+1\right)^{2}\left(a_{1} t+1\right)} .
$$

From equations (6.18) and (6.25) and (6.35), the expansion acceleration $a$ is also expressed as

$$
a=r \frac{\left(\frac{\mathrm{d}^{2} E_{R}}{\mathrm{~d} t^{2}}\right)}{E_{R}} .
$$

From equations (6.36) and (6.40), the acceleration parameter $A_{P}$ is also expressed as

$$
A_{P}=\frac{\left(\frac{\mathrm{d}^{2} E_{R}}{\mathrm{~d} t^{2}}\right)}{E_{R}} .
$$

From equations $(6.39)$ and $(6.41), \frac{\left(\frac{\mathrm{d}^{2} E_{R}}{\mathrm{~d} t^{2}}\right)}{E_{R}}$ is expressed as

$$
\frac{\left(\frac{\mathrm{d}^{2} E_{R}}{\mathrm{~d} t^{2}}\right)}{E_{R}}=2 \times \frac{a_{1} b_{2}^{2} t^{3}+3 b_{2}^{2} t^{2}-3 a_{1} b_{2} t+3 b_{1} b_{2} t-b_{2}-a_{1} b_{1}+b_{1}^{2}}{\left(b_{2} t^{2}+b_{1} t+1\right)^{2}\left(a_{1} t+1\right)} .
$$

We derive the deceleration parameter. The deceleration parameter $q$ is defined as

$$
q=-\frac{\frac{\mathrm{d}^{2} A(t)}{\mathrm{d} t^{2}} \times A(t)}{\left(\frac{\mathrm{d} A(t)}{\mathrm{d} t}\right)^{2}}
$$

where $A(t)$ is the dimensionless scale factor. To distinguish the dimensionless scale factor from the acceleration $a$ used in this paper, we use the notations $A(t)$ and $A(Z)$ for the dimensionless scale factor. Using $A(t)$, the Hubble parameter $H$ is expressed as

$$
H=\frac{\left(\frac{\mathrm{d} A(t)}{\mathrm{d} t}\right)}{A(t)} .
$$

From the comparison between equation (6.33) and equation (6.44), the dimensionless scale factor $A(t)$ is the spacetime expansion rate $E_{R}$ in this paper.

$$
E_{R}=A(t) .
$$

Therefore, using $E_{R}$, the deceleration parameter $q$ is expressed as

$$
q=-\frac{\frac{\mathrm{d}^{2} E_{R}}{\mathrm{~d} t^{2}} \times E_{R}}{\left(\frac{\mathrm{d} E_{R}}{\mathrm{~d} t}\right)^{2}} .
$$

From equations (6.46) and (6.33) and (6.41), the deceleration parameter $q$ is expressed as 


$$
q=-\frac{\left(\frac{\mathrm{d}^{2} E_{R}}{\mathrm{~d} t^{2}}\right) / E_{R}}{\left(\left(\frac{\mathrm{d} E_{R}}{\mathrm{~d} t}\right) / E_{R}\right)^{2}}=-\frac{A_{P}}{H^{2}} .
$$

From equations (6.31) and (6.39) and (6.47), the deceleration parameter $q$ is expressed as

$$
q=-\frac{A_{P}}{H^{2}}=-2 \times \frac{\left(a_{1} b_{2}^{2} t^{3}+3 b_{2}^{2} t^{2}-3 a_{1} b_{2} t+3 b_{1} b_{2} t-b_{2}-a_{1} b_{1}+b_{1}^{2}\right)\left(a_{1} t+1\right)}{\left(-a_{1} b_{2} t^{2}-2 b_{2} t+a_{1}-b_{1}\right)^{2}} .
$$

In this way, the equations on the spacetime expansion are derived.

\subsection{Calculation of Unknown $a_{1}, b_{1}$ and $b_{2}$}

By specifying the conditions, we calculate the unknowns $a_{1}, b_{1}$ and $b_{2}$.

First of all, we determine the conditions to specify. There are three unknowns in the Hubble parameter $H$ and the acceleration parameter $A_{P}: a_{1}, b_{1}$ and $b_{2}$. For this reason, by specifying three conditions, we can calculate the unknowns $a_{1}, b_{1}$ and $b_{2}$. In other words, we can specify only three conditions. Therefore, it is important that these three conditions are certain. We specify the following two sets of three conditions.

- Acceleration period matching conditions:

$$
\begin{gathered}
H\left(t=t_{1}\right)=H_{1}=H_{0} . \\
A_{P}(t=0)=0 . \\
H(t=0)=H_{00} .
\end{gathered}
$$

- Deceleration period matching conditions:

$$
\begin{aligned}
& N_{R}\left(t=t_{0}\right)=0 . \\
& A_{P}(t=0)=0 . \\
& H(t=0)=H_{00} .
\end{aligned}
$$


As mentioned earlier, $t=t_{1}$ is the current time, $t=t_{0}$ is the universe's birth time, and $t=0$ is the time when the deceleration expansion changes to the acceleration expansion. $H_{1}$ and $H_{0}$ are the Hubble constant at $t=t_{1}$, and $H_{00}$ is the Hubble constant at $t=0$. The two sets differ only in equation (6.49) and equation (6.52). Because of these conditions, if the time when $A_{P}=0$ is set to $t=0$, it is easy to calculate.

We calculate the unknowns $a_{1}, b_{1}$ and $b_{2}$ under the acceleration period matching conditions. From equations (6.51) and (6.31), the unknown $a_{1}$ is expressed as

$$
a_{1}=H_{00}+b_{1}
$$

From equations (6.39) and (6.50) and (6.53), the unknown $b_{2}$ is expressed as

$$
b_{2}=-H_{00} b_{1} .
$$

From equations (6.31) and (6.49) and (6.53) and (6.54), the unknown $b_{1}$ is expressed as

$$
b_{1}=\frac{-H_{00}^{2} H_{1} t_{1}^{2}-H_{00}^{2} t_{1}+2 H_{1}-2 H_{00} \pm H_{00} \sqrt{H_{00}^{2} H_{1}^{2} t_{1}^{4}+2 H_{00}^{2} H_{1} t_{1}^{3}+\left(4 H_{00} H_{1}+H_{00}^{2}\right) t_{1}^{2}+\left(4 H_{00}-4 H_{1}\right) t_{1}}}{2 H_{00} H_{1} t_{1}^{2}+\left(2 H_{00}-2 H_{1}\right) t_{1}} .
$$

We calculate the unknowns $a_{1}, b_{1}$ and $b_{2}$ under the deceleration period matching conditions. From equations (6.14) and (6.52), the unknown $a_{1}$ is expressed as

$$
a_{1}=-\frac{1}{t_{0}}
$$

From equations (6.31) and (6.51), the unknown $b_{1}$ is expressed as

$$
b_{1}=a_{1}-H_{00} .
$$

From equations (6.39) and (6.50), the unknown $b_{2}$ is expressed as

$$
b_{2}=-a_{1} b_{1}+b_{1}^{2} .
$$

In this way, by specifying the conditions, the unknowns $a_{1}, b_{1}$ and $b_{2}$ are calculated.

\subsection{Equations in the $\Lambda \mathrm{CDM}$ Model}

We show the equations in the $\Lambda C D M$ model. From equation (6.45), the spacetime expansion rate $E_{R}$ in this paper is the dimensionless scale factor $A(t)$ in the $\Lambda$ CDM model. The equations in the $\Lambda \mathrm{CDM}$ model are used to compare with the calculations of this paper and to obtain the necessary data.

The dimensionless scale factor $A(t)$ in the $\Lambda$ CDM model is expressed as 


$$
\begin{gathered}
A(t)=\left(\frac{\Omega_{m}}{\Omega_{\Lambda}}\right)^{\frac{1}{3}} \sinh ^{\frac{2}{3}}\left(\frac{t}{t_{A}}\right), \\
t_{A}=\frac{2}{3 H_{0} \sqrt{\Omega_{\Lambda}}}, \\
A(Z)=\frac{1}{Z+1},
\end{gathered}
$$

where $\Omega_{m}$ is the matter density parameter, $\Omega_{\Lambda}$ is the dark energy density parameter, $Z$ is a redshift.

The Hubble parameter in the $\Lambda \mathrm{CDM}$ model is expressed as

$$
\begin{gathered}
H(Z)=H_{0} \sqrt{\Omega_{m}(1+Z)^{3}+\Omega_{\Lambda}}, \\
H(t)=H_{0} \sqrt{\frac{\Omega_{m}}{A(t)^{3}}+\Omega_{\Lambda}} .
\end{gathered}
$$

From the Friedmann acceleration equation, the deceleration parameter in the $\Lambda \mathrm{CDM}$ model is expressed as

$$
\begin{gathered}
q=\frac{\frac{1}{2} \Omega_{m}(1+Z)^{3}-\Omega_{\Lambda}}{\Omega_{m}(1+Z)^{3}+\Omega_{\Lambda}} \\
q=\frac{\frac{1}{2} \Omega_{m} / A(t)^{3}-\Omega_{\Lambda}}{\Omega_{m} / A(t)^{3}+\Omega_{\Lambda}}
\end{gathered}
$$

If spacetime expands with constant mass, the matter density decreases in inverse proportion to the cube of the distance. Therefore, $\Omega_{m}$ is multiplied by $(1+Z)^{3}=\frac{1}{A(t)^{3}}$.

Since $t=0$ is the time when $A_{P}=0$, not the current time, the spacetime expansion rate $E_{R}(Z)$ in this paper is expressed as

$$
E_{R}(Z)=\frac{Z_{00}+1}{Z+1}
$$

where $Z_{00}$ is the redshift at $t=0$. Since the redshift $Z$ at the current time of $t=t_{1}$ is zero, the spacetime expansion rate $E_{R}\left(t=t_{1}\right)$ at $t=t_{1}$ is expressed as

$$
E_{R}\left(t=t_{1}\right)=Z_{00}+1 \text {. }
$$

In this way, the equations in the $\Lambda \mathrm{CDM}$ model are shown. 


\subsection{Data Required to Obtain the Unknowns $a_{1}, b_{1}$ and $b_{2}$}

We show the data required to obtain the unknowns $a_{1}, b_{1}$ and $b_{2}$.

From equations (6.53) to (6.58), the required data are the following four: $H\left(t=t_{1}\right)=$ $H_{1}=H_{0}, H(t=0)=H_{00}, t=t_{1}, t=t_{0}$. However, of the four data, only $H_{1}=H_{0}$ has observation data. The remaining three data can only be derived from the equations in the $\Lambda$ CDM model.

From the $\Lambda$ CDM model, we discuss the data required to obtain the three data.

From equation (6.59), time $t$ is expressed as

$$
t=t_{A} \times \operatorname{arsinh}\left(\left(A(t)\left(\frac{\Omega_{\Lambda}}{\Omega_{m}}\right)^{\frac{1}{3}}\right)^{\frac{3}{2}}\right) .
$$

Substituting $A(t)=1$ into equation (6.68), the time $t$ at that time is the age of the universe $t=t_{1}+\left|t_{0}\right|$.

Substituting equation (6.61) into equation (6.68), it is expressed as

$$
t=t_{A} \times \operatorname{arsinh}\left(\left(\frac{1}{Z+1}\left(\frac{\Omega_{\Lambda}}{\Omega_{m}}\right)^{\frac{1}{3}}\right)^{\frac{3}{2}}\right) .
$$

Substituting $Z=Z_{00}$ into equation (6.69), the time $t$ at that time is the time $\left|t_{0}\right|$ when the deceleration expansion changes to the acceleration expansion. From $\left|t_{0}\right|$ and $t_{1}+\left|t_{0}\right|$, we can obtain the current time $t_{1}$.

We derive the redshift $Z_{00}$ at the time when the deceleration expansion changes to the acceleration expansion. Substituting $q=0$ into equation (6.64), the redshift $Z$ at that time is the redshift $Z_{00}$. Therefore, the redshift $Z_{00}$ is expressed as

$$
Z_{00}=\left(2 \frac{\Omega_{\Lambda}}{\Omega_{m}}\right)^{\frac{1}{3}}-1
$$

Substituting $Z=Z_{00}$ into equation (6.62), we can obtain $H_{00}$.

From the above, the data required to obtain the three data are $\Omega_{m}$ and $\Omega_{\Lambda}$.

Observations show that $H_{1}\left(=H_{0}\right)$ and $\Omega_{\Lambda}$ and $\Omega_{m}$ are expressed as [16]

$$
\begin{gathered}
H_{1}=H_{0}=67.74 \pm 0.46 \mathrm{~km} / \mathrm{s} / \mathrm{Mpc}, \\
\Omega_{\Lambda}=0.6911 \pm 0.0062 \\
\Omega_{m}=0.3089 \pm 0.0062 .
\end{gathered}
$$

Therefore, from the equations in the $\Lambda \mathrm{CDM}$ model and the observations data, the data required to obtain the unknowns $a_{1}, b_{1}$ and $b_{2}$ are expressed as 


$$
\begin{gathered}
H_{1}=H_{0}=67.74 \pm 0.46 \mathrm{~km} / \mathrm{s} / \mathrm{Mpc}, \\
H_{00}=97.54 \mathrm{~km} / \mathrm{s} / \mathrm{Mpc}, \\
t_{1}=6.180 \text { billion years }, \\
t_{0}=-7.622 \text { billion years } .
\end{gathered}
$$

\subsection{Calculation Results and Graphs in the Spacetime Expansion}

We show the calculation results under the acceleration period matching conditions as follows:

$$
\begin{gathered}
a_{1}=110.1 \mathrm{~km} / \mathrm{s} / \mathrm{Mpc}, \\
b_{1}=12.52 \mathrm{~km} / \mathrm{s} / \mathrm{Mpc}, \\
b_{2}=-1222 \mathrm{~km}^{2} / \mathrm{s}^{2} / \mathrm{Mpc}^{2} .
\end{gathered}
$$

We show the calculation results under the deceleration period matching conditions as follows:

$$
\begin{gathered}
a_{1}=128.3 \mathrm{~km} / \mathrm{s} / \mathrm{Mpc}, \\
b_{1}=30.74 \mathrm{~km} / \mathrm{s} / \mathrm{Mpc}, \\
b_{2}=-2999 \mathrm{~km}^{2} / \mathrm{s}^{2} / \mathrm{Mpc}^{2} .
\end{gathered}
$$

Graphs based on these values are shown in figures 5 to 9 . 


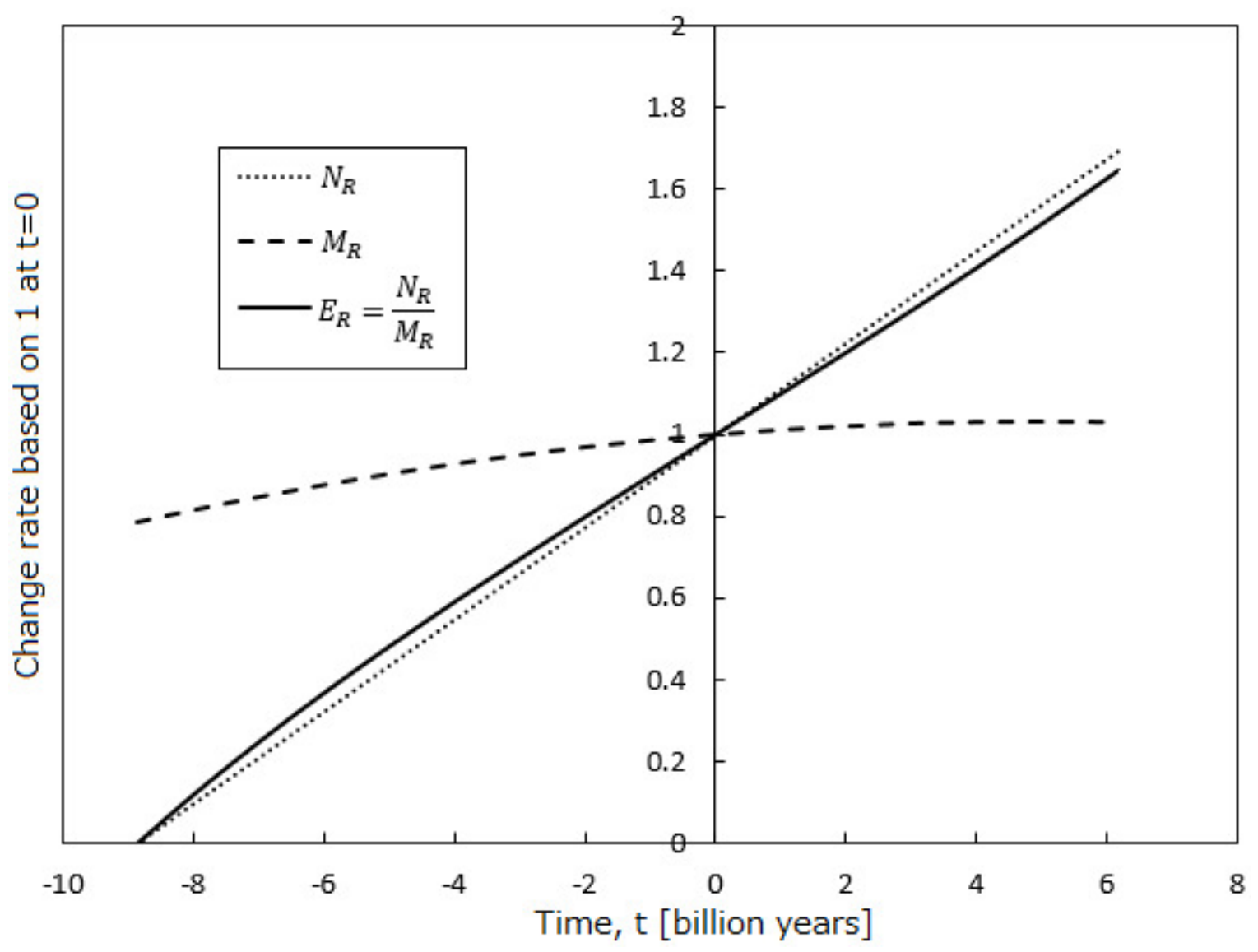

Figure 5: Graphs of $N_{R}$ and $M_{R}$ and $E_{R}=\frac{N_{R}}{M_{R}}$ under the acceleration period matching conditions. $N_{R}$ is the spacetime particle number change rate, $M_{R}$ is the mass change rate of ordinary matter, and $E_{R}$ is the spacetime expansion rate. All are change rates from the reference time $t=0$, and all are 1 at $t=0$. 


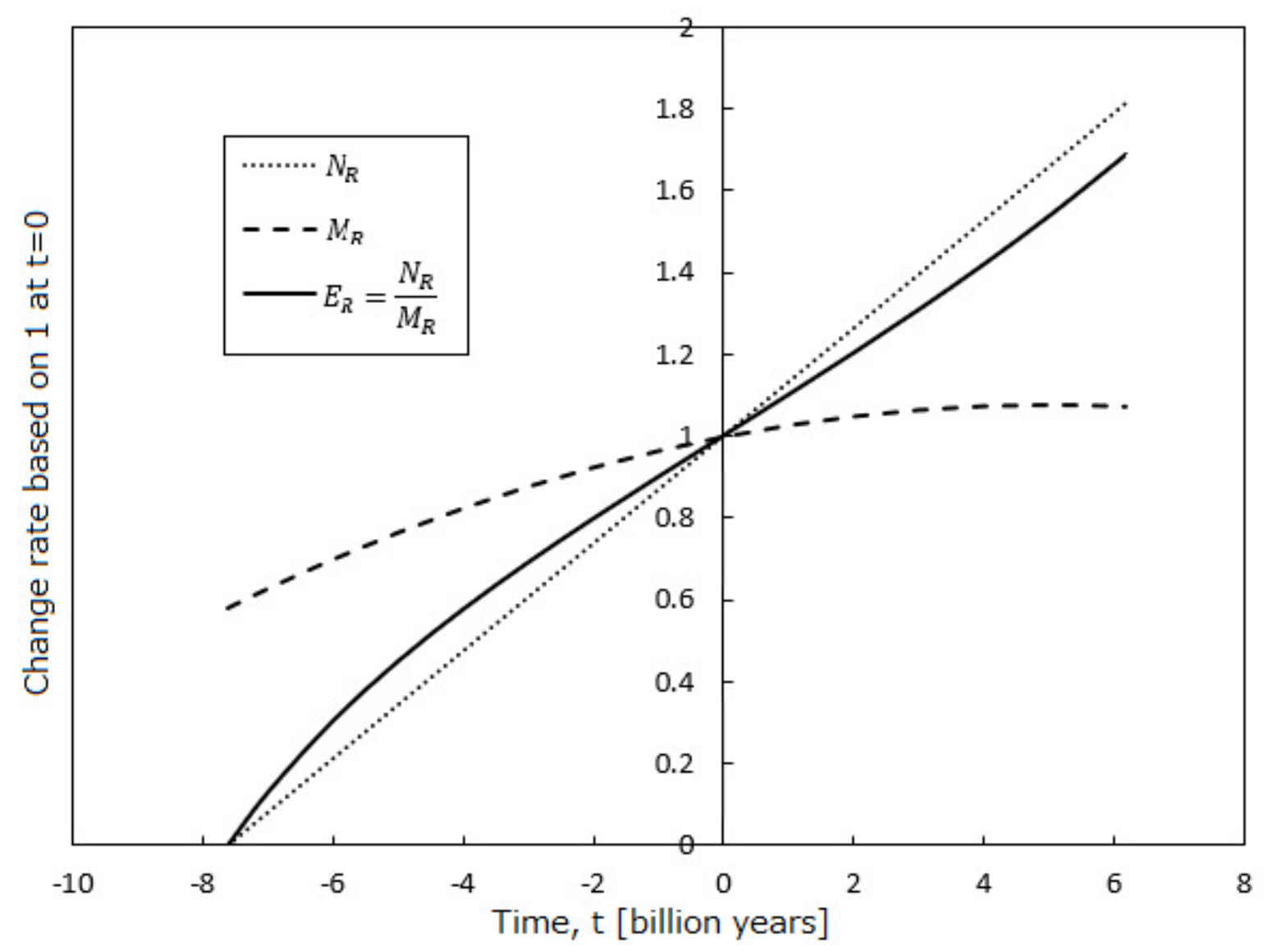

Figure 6: Graphs of $N_{R}$ and $M_{R}$ and $E_{R}=\frac{N_{R}}{M_{R}}$ under the deceleration period matching conditions. $N_{R}$ is the spacetime particle number change rate, $M_{R}$ is the mass change rate of ordinary matter, and $E_{R}$ is the spacetime expansion rate. All are change rates from the reference time $t=0$, and all are 1 at $t=0$. 


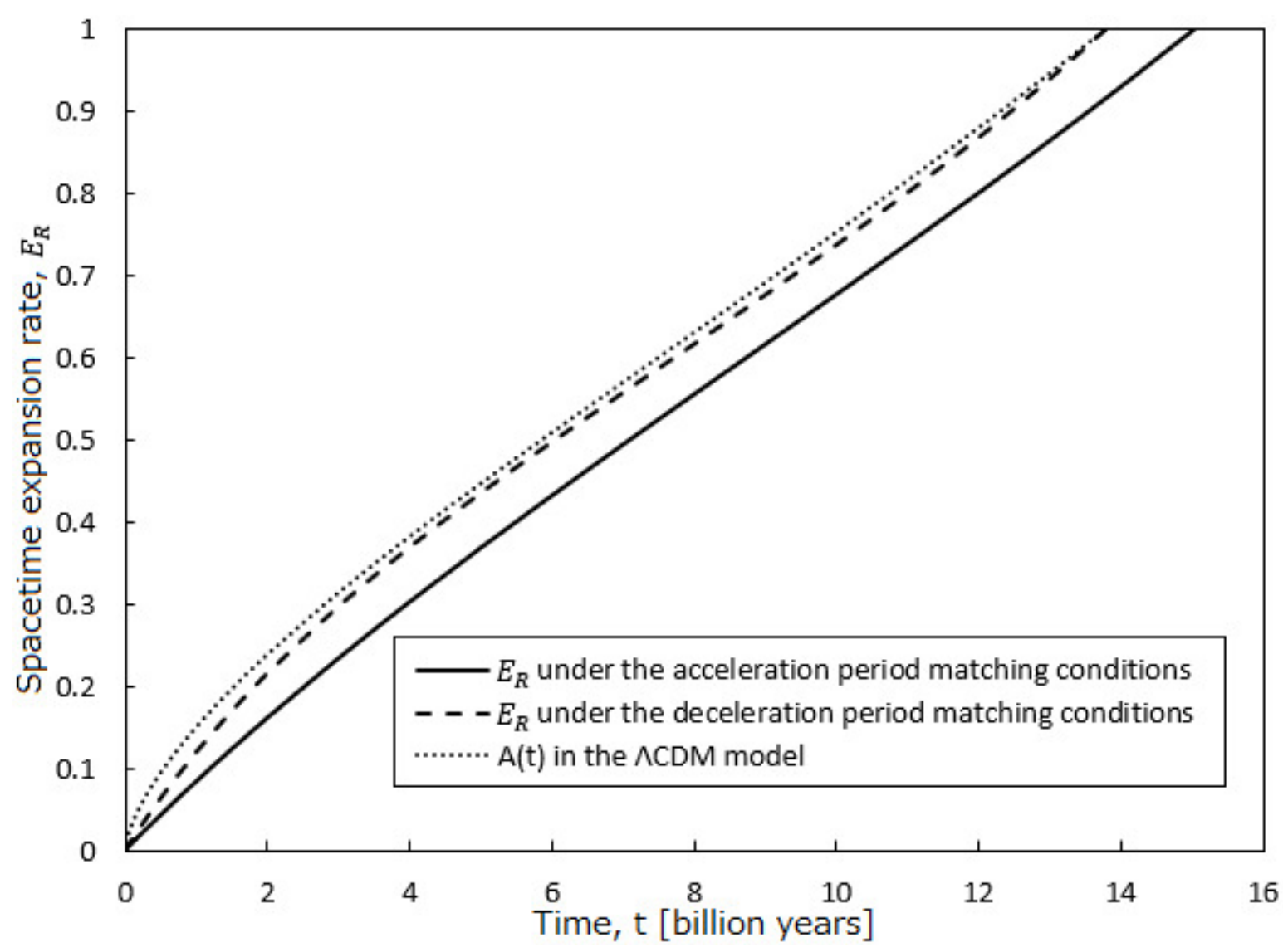

Figure 7: Comparison graphs of $E_{R}$ and $A(t) . E_{R}$ is the spacetime expansion rate in this paper, and $A(t)$ is the dimensionless scale factor in the $\Lambda \mathrm{CDM}$ model, and $E_{R}$ is equal to $A(t)\left(E_{R}=A(t)\right)$. The origin coordinates are all the same, and the time when $E_{R}=1$ is each current time. 


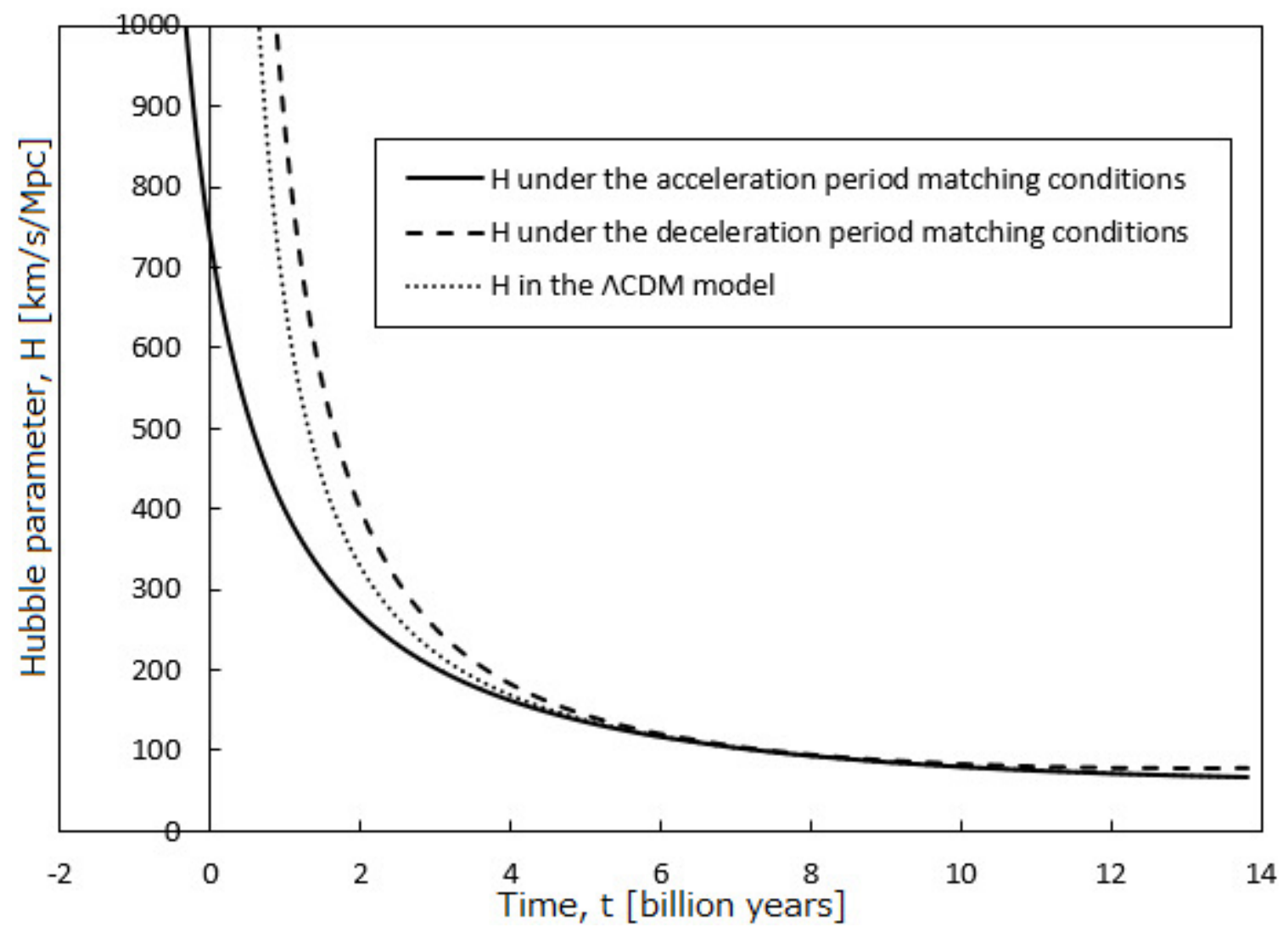

Figure 8: Comparison graphs of the Hubble parameter $H$ including the $\Lambda$ CDM model. The current time is all the same. 


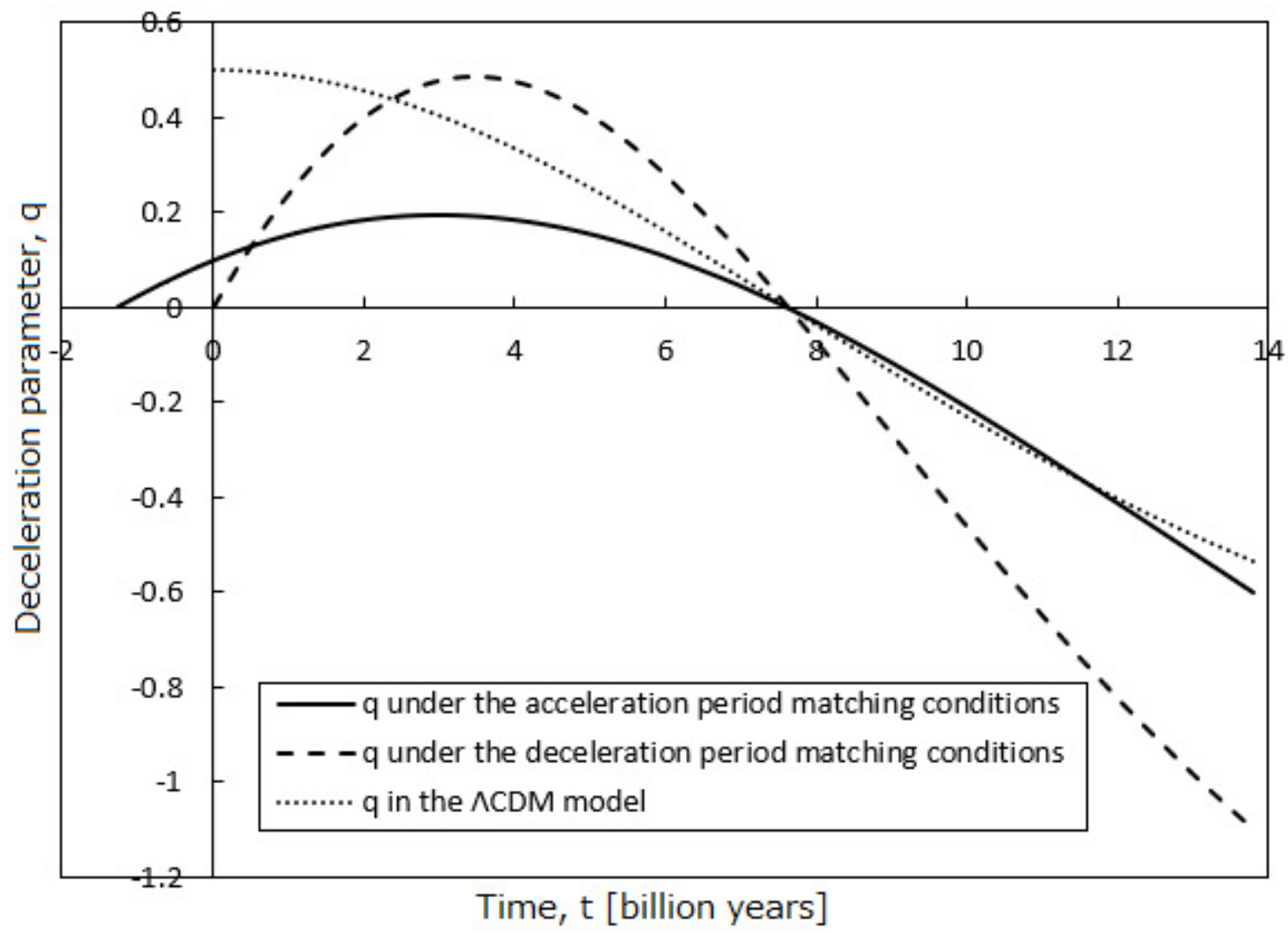

Figure 9: Comparison graphs of the deceleration parameter $q$ including the $\Lambda$ CDM model. The current time is all the same.

In this way, the calculation results and graphs in the spacetime expansion are shown.

\subsection{Discussion of the Spacetime Expansion}

We discuss the calculation results in the spacetime expansion.

First of all, we discuss the spacetime particle number change rate $N_{R}$. Both conditions have increased in proportion to time since the birth of the universe. However, the slope of the deceleration period matching condition is larger than that of the acceleration period matching condition. The time when $N_{R}=0$ under the acceleration period matching condition is expressed as

$$
t=t_{0}=-8.884 \text { billion years }=15.06 \text { billion years ago } .
$$


The time when $N_{R}=0$ under the deceleration period matching condition is expressed as

$$
t=t_{0}=-7.622 \text { billion years }=13.80 \text { billion years ago } .
$$

Since the spacetime particle number $N$ represents spacetime, the time when the spacetime particle number $N$ becomes 0 is the moment of the birth of the universe. Therefore, the age of the universe is 15.06 billion years or 13.80 billion years. If the age of the universe is 15.06 billion years, it can explain the age of $14.46 \pm 0.8$ billion years of the oldest star HD 140283 [17].

We discuss the mass change rate $M_{R}$ of ordinary matter. In the deceleration expansion period of $t<0$, the mass is increasing. In the acceleration expansion period of $t \geqq 0$, the mass increase begins to stop, then the mass change becomes almost constant, and then the mass begins to decrease slightly. Both conditions have the same characteristics. However, the mass under the deceleration period matching condition increases rapidly.

Notable things are the following.

- In the case of the acceleration period matching condition.

$$
\begin{gathered}
M_{R}\left(t=t_{1}=6.180 \text { billion years }\right)=1.030 . \\
M_{R}\left(t=t_{0}=-8.884 \text { billion years }\right)=0.7854 .
\end{gathered}
$$

- In the case of the deceleration period matching condition.

$$
\begin{gathered}
M_{R}\left(t=t_{1}=6.180 \text { billion years }\right)=1.075 . \\
M_{R}\left(t=t_{0}=-7.622 \text { billion years }\right)=0.5781 .
\end{gathered}
$$

The time $t_{1}$ is the current time, and the time $t_{0}$ is the time of the birth of the universe. In both conditions, the mass change rate $M_{R}$ of ordinary matter is not zero at the time of the birth of the universe. That is, the mass has existed from the moment of the birth of the universe. From the Big Bang theory, the universe began to expand from one point, so the universe was born from a supermassive black hole.

The time when $M_{R}=0$ is expressed as follows.

- In the case of the acceleration period matching condition.

$$
t=-23.41 \text { billion years }=29.59 \text { billion years ago } .
$$




$$
t=33.43 \text { billion years }=27.25 \text { billion years later } .
$$

- In the case of the deceleration period matching condition.

$$
\begin{aligned}
& t=-13.53 \text { billion years }=19.71 \text { billion years ago } . \\
& t=23.56 \text { billion years }=17.38 \text { billion years later } .
\end{aligned}
$$

Before the birth of the universe, there should be no spacetime itself, so the time before that is unnatural. However, in the calculation, the mass has continued to increase even before the birth of the universe. This conclusion is explained in section 12 .

We discuss the spacetime expansion rate $E_{R}$. The graph of the spacetime expansion rate $E_{R}$ including $A(t)$ of the $\Lambda \mathrm{CDM}$ model is shown in figure 7 . Since the age of the universe is the same, the $E_{R}$ of the deceleration period matching condition and the $A(t)$ of the $\Lambda$ CDM model are almost the same. However, since the age of the universe is different, only the $E_{R}$ of the acceleration period matching condition is different.

We discuss the Hubble parameter $H$. The graph of the Hubble parameter $H$ including the $\Lambda \mathrm{CDM}$ model is shown in figure 8 . In the acceleration period, the Hubble parameter $H$ of the acceleration period matching condition is almost the same as that of the $\Lambda \mathrm{CDM}$ model, but only that of the deceleration period matching condition is different. In the deceleration period, each Hubble parameter $H$ is different.

We discuss the deceleration parameter $q$. The graph of the deceleration parameter $q$ including the $\Lambda \mathrm{CDM}$ model is shown in figure 9. As can be seen from the figure, the graph of each deceleration parameter $q$ is different.

We discuss the change in spacetime expansion.

First, from $E_{R}=\frac{N_{R}}{M_{R}}=\frac{a_{1} t+1}{b_{2} t^{2}+b_{1} t+1}$ of equation (6.16), the increase of spacetime particles number is always constant, so it is the mass change of ordinary matter that changes the spacetime expansion. During the deceleration expansion period, the spacetime expansion is decelerating because the mass increase speed is fast. During the acceleration expansion period, the spacetime expansion is accelerating because the mass increase speed is slow or decreasing. Now the mass has decreased slightly. Therefore, we conclude that the acceleration expansion at present is caused by the mass reduction of ordinary matter. For this reason, dark energy is a virtual negative mass by the mass reduction of ordinary matter.

Second, the first law of thermodynamics suggests that spacetime expansion changes with mass changes. From the first law of thermodynamics, when space expands, if the pressure is outward, work is done to outside. This means that internal energy is consumed. The dark energy of the acceleration expansion can be regarded as the pressure pushing space outward. 
Therefore, the first law of thermodynamics suggests that in the acceleration expansion of spacetime, the mass of ordinary matter that is the internal energy is consumed. Conversely, when space expands, if the pressure is inward, work is done from outside. This means that energy is supplied from outside. The energy of the deceleration expansion can be regarded as the pressure pushing space inward. Therefore, the first law of thermodynamics suggests that in the deceleration expansion of spacetime, energy is supplied from outside and the mass of ordinary matter increases.

Third, since the positive mass causes the deceleration expansion, the dark energy of the acceleration expansion is always equivalent to the negative mass under any assumption. However, the existence of negative mass has not been confirmed. Therefore, dark energy is a virtual negative mass by the mass reduction of ordinary matter.

From the above, we conclude that the change in spacetime expansion is caused by the change in the mass of ordinary matter.

Note that the inter-matter radius changes with the gravity of other matter and so on, but the spacetime radius changes only with the spacetime particle number $N$ and the mass $M$ of the expansion source. Moreover, from premise 6.2 and assumption 6.6, we assume that the inter-matter radius changes only in conjunction with the spacetime radius. This is to say that the change in the inter-matter radius is equivalent to the expansion of spacetime. If the inter-matter radius changes by the gravity of other matter, that change is not a spacetime expansion, but just a change in position. Therefore, it is not considered here.

In this way, we can explain spacetime expansion from the equation of state of spacetime particles.

\section{Force Received by Mass}

In this section, we compare the three forces received by mass. The first is the gravity of ordinary matter, which is inversely proportional to the square of distance. The second is the gravity of spacetime particles (generally dark matter), which is inversely proportional to distance. The third is the spacetime expansion acceleration force (generally dark energy), which is proportional to distance.

\subsection{Spacetime Expansion Acceleration Force}

We explain the spacetime expansion acceleration force.

First of all, we show the equation of the spacetime expansion acceleration force. From equations (6.28) and (6.36), the expansion speed $v$ and the expansion acceleration $a$ of the inter-matter radius $r$ are expressed as $v=r H$ and $a=r A_{P}$. The inter-matter radius $r$ is the

radius from the center of the matter of the expansion source to another matter with active 
gravitational mass. Since the inter-matter radius $r$ expands at the expansion speed $v$, matter receives a force $F$ by the expansion acceleration $a$. A force $F$ by the expansion acceleration $a$ is called the spacetime expansion acceleration force. The spacetime expansion acceleration force $F$ is expressed as

$$
F=m a=m r A_{P} .
$$

We explain the force of equation (7.1). From equation (6.37), the acceleration parameter $A_{P}$ is expressed as $A_{P}=\frac{a}{r}=\frac{a_{\max }}{r_{\max }}$. The radius $r_{\max }$ is the maximum spacetime radius. The acceleration $a_{\max }$ is the expansion acceleration of $r_{\max }$. Therefore, the force of equation (7.1) is the force received by the expansion acceleration of spacetime.

The spacetime expansion acceleration force can be explained as the opposite force of an inertial force. An inertial force is a force that is received when the translational velocity of the entire spacetime particle distribution is constant and the velocity of an object changes. Conversely, the spacetime expansion acceleration force is a force that is received when the velocity of an object is constant and the speed of the radius of the spacetime particle distribution changes.

From equation (7.1), when $A_{P}>0$ and spacetime expansion is acceleration expansion, the spacetime expansion acceleration force is a repulsive force. When $A_{P}<0$ and spacetime expansion is deceleration expansion, the spacetime expansion acceleration force is an attractive force. Therefore, the spacetime expansion acceleration force is not always a repulsive force.

We express $r, v, a$ and $F$ based on the spacetime expansion rate $E_{R}$ and compare them. Since the spacetime radius $r_{\max }$ and the inter-matter radius $r$ expand at the same spacetime expansion rate $E_{R}$, the inter-matter radius $r$ is expressed as

$$
r=r(t=0) \times E_{R},
$$

where $r(t=0)$ is the inter-matter radius $r$ at $t=0$. From equations (6.32) and (7.2), the expansion speed $v$ of the inter-matter radius $r$ is expressed as

$$
v=r(t=0) \times \frac{\mathrm{d} E_{R}}{\mathrm{~d} t} .
$$

From equations (6.40) and (7.3), the expansion acceleration $a$ of the inter-matter radius $r$ is expressed as

$$
a=r(t=0) \times \frac{\mathrm{d}^{2} E_{R}}{\mathrm{~d} t^{2}} .
$$

From equation (7.4), the spacetime expansion acceleration force $F$ is expressed as

$$
F=m a=m r(t=0) \times \frac{\mathrm{d}^{2} E_{R}}{\mathrm{~d} t^{2}} .
$$


Differentiating the inter-matter radius $r$ yields the expansion speed $v$ of the inter-matter radius $r$, and differentiating the expansion speed $v$ yields the expansion acceleration $a$ of the inter-matter radius $r$. In this way, in the calculation of the spacetime expansion, if the inter-matter radius $r$ is expressed as $r=r(t=0) \times E_{R}$, we can calculate it correctly.

We show the spacetime particle number density of the spacetime expansion acceleration force. Substituting $a=r A_{P}$ into the Unruh temperature $T_{u}$ of equation (2.5), $T_{u}$ is expressed as

$$
T_{u}=\frac{\mathrm{k}_{\mathrm{sp}}}{\mathrm{k}_{\mathrm{B}}} \times r A_{P} .
$$

From equations (2.4) and (7.6), the temperature $T$ by the spacetime expansion acceleration force is expressed as

$$
T=\frac{m}{M} \times T_{s p} \times \frac{\mathrm{k}_{\mathrm{sp}}}{\mathrm{k}_{\mathrm{B}}} \times r A_{P} .
$$

Since the area $S$ is the area where the acceleration $a$ is constant, the area $S$ is the sphere surface area $S=4 \pi r^{2}$. Therefore, from equations (2.9) and (7.1) and (7.7), the spacetime particle number density of the spacetime expansion acceleration force is expressed as

$$
\frac{\mathrm{d} N}{\mathrm{~d} V}=\frac{M}{4 \pi r^{2} \mathrm{k}_{s p} T_{s p}} .
$$

Equation (7.8) is the same as the spacetime particle number density of gravity. The general equation of the spacetime particle number density is expressed as equation (2.14). Therefore, if the area $S$ at which the acceleration $a$ is constant is the same, the spacetime particle number densities are all the same.

\subsection{Comparison of the Gravity of Ordinary Matter, the Gravity of Spacetime Particles and the Spacetime Expansion Acceleration Force}

We compare three forces: the gravity of ordinary matter, the gravity of spacetime particles and the spacetime expansion acceleration force.

From equations (3.8) and (3.9) and (7.1), the three forces in Newtonian gravity form are expressed as

$$
\begin{aligned}
& F_{1}=-\frac{\mathrm{G} M m}{r^{2}}, \\
& F_{2}=-\frac{\mathrm{G} M m}{r \times \mathrm{r}_{\mathrm{e}}},
\end{aligned}
$$




$$
F_{3}=+m r A_{P},
$$

where $F_{1}$ is the gravity of ordinary matter, $F_{2}$ is the gravity of spacetime particles, and $F_{3}$ is the spacetime expansion acceleration force. In Newtonian gravity form, force $F$ received by mass $m$ is expressed as

$$
F=-\frac{\mathrm{G} M m}{r^{2}}-\frac{\mathrm{G} M m}{r \times \mathrm{r}_{\mathrm{e}}}+m r A_{P} .
$$

From equations (2.9) and (2.12) and (3.6) and (7.7), the three forces in thermodynamic gravity form are expressed as

$$
\begin{aligned}
& F_{1}=S \times \frac{\mathrm{d} N}{\mathrm{~d} V} \times \mathrm{k}_{\mathrm{B}} \times \frac{m}{M} \times T_{s p} \times \frac{\mathrm{k}_{\mathrm{sp}}}{\mathrm{k}_{\mathrm{B}}} \times\left(-\frac{\mathrm{G} M}{r^{2}}\right), \\
& F_{2}=S \times \frac{\mathrm{d} N}{\mathrm{~d} V} \times \mathrm{k}_{\mathrm{B}} \times \frac{m}{M} \times T_{s p} \times \frac{\mathrm{k}_{\mathrm{sp}}}{\mathrm{k}_{\mathrm{B}}} \times\left(-\frac{\mathrm{G} M}{r \times \mathrm{r}_{\mathrm{e}}}\right), \\
& F_{3}=S \times \frac{\mathrm{d} N}{\mathrm{~d} V} \times \mathrm{k}_{\mathrm{B}} \times \frac{m}{M} \times T_{s p} \times \frac{\mathrm{k}_{\mathrm{sp}}}{\mathrm{k}_{\mathrm{B}}} \times\left(+r A_{P}\right) .
\end{aligned}
$$

In thermodynamic gravity form, force $F$ received by mass $m$ is expressed as

$$
F=S \times \frac{\mathrm{d} N}{\mathrm{~d} V} \times \mathrm{k}_{\mathrm{B}} \times \frac{m}{M} \times T_{s p} \times \frac{\mathrm{k}_{\mathrm{sp}}}{\mathrm{k}_{\mathrm{B}}} \times\left(-\frac{\mathrm{G} M}{r^{2}}-\frac{\mathrm{G} M}{r \times \mathrm{r}_{\mathrm{e}}}+r A_{P}\right) .
$$

We show the distance $r_{e 2}$ where the sum of gravity and the spacetime expansion acceleration force becomes zero at the current time. Since the $A_{P}$ at the current time is $A_{P}>0$, the distance $r_{e 2}$ is the distance where $F=0$. From $F=0$, equation (7.12) is expressed as

$$
\frac{\mathrm{G} M m}{r_{e 2}^{2}}+\frac{\mathrm{G} M m}{r_{e 2} \times \mathrm{r}_{\mathrm{e}}}=m r_{e 2} A_{P} .
$$

If we calculate $r_{e 2}$ exactly from equation (7.17), it becomes a cubic equation, which complicates the calculation. From observations, in a galaxy, gravity is stronger than repulsion. That is, the distance $r_{e 2}$ is sufficiently larger than $r_{e}$. From $r_{e 2}>>r_{e}$, equation (7.17) can be approximated as

$$
\frac{\mathrm{G} M m}{r_{e 2} \times \mathrm{r}_{\mathrm{e}}} \fallingdotseq m r_{e 2} A_{P}
$$

Therefore, the distance $r_{e 2}$ is expressed as

$$
r_{e 2}=\sqrt{\frac{\mathrm{G} M}{\mathrm{r}_{\mathrm{e}} A_{P}}}
$$


We show the concrete value of $r_{e 2}$ in the Milky Way. We use the mass of equation (3.18) for the mass $M$ of ordinary matter in the Milky Way, $A_{P}$ of the acceleration period matching condition for $A_{P}$, and the current time of $t=t_{1}=6.180$ billion years for the time $t$ of $A_{P}$. From these and equation (7.19), the distance $r_{e 2}$ in the Milky Way is expressed as

$$
r_{e 2} \fallingdotseq 6.88 \times 10^{23} \text { meters }=72.7 \text { million light }- \text { years } .
$$

Since the distance $r_{e}$ is $r_{e}=5.34 \times 10^{18}$ meters from equation (3.24), we can see that $r_{e 2}$ is sufficiently larger than $r_{e}$.

We show the distance change of the three forces at the current time.

In the case of short-range of $r<<\mathrm{r}_{\mathrm{e}}<<r_{e 2}$, force $F$ received by mass can be approximated as

$$
F \fallingdotseq F_{1}=-\frac{\mathrm{G} M m}{r^{2}}
$$

In the case of medium-range of $\mathrm{r}_{\mathrm{e}}<<r<<r_{e 2}$, force $F$ received by mass can be approximated as

$$
F \fallingdotseq F_{2}=-\frac{\mathrm{G} M m}{r \times \mathrm{r}_{\mathrm{e}}}
$$

In the case of long-range of $\mathrm{r}_{\mathrm{e}}<<r_{e 2}<<r$, force $F$ received by mass can be approximated as

$$
F \fallingdotseq F_{3}=+m r A_{P}
$$

In this way, the dominant force of the three forces changes depending on the distance.

\subsection{Mass Densities of Ordinary Matter, Dark Matter and Dark Energy}

We show the mass densities of ordinary matter, dark matter and dark energy from observations.

When we rearrange equation (7.12), it is expressed as

$$
F=-\frac{\mathrm{G} m}{r^{2}}\left(M+M \frac{r}{\mathrm{r}_{\mathrm{e}}}-A_{P} \frac{r^{3}}{\mathrm{G}}\right)
$$

From equation (7.24), the mass $M_{1}$ of ordinary matter is expressed as

$$
M_{1}=+M,
$$

the mass $M_{2}$ of dark matter is expressed as 


$$
M_{2}=+M \frac{r}{\mathrm{r}_{\mathrm{e}}}
$$

the mass $M_{3}$ of dark energy is expressed as

$$
M_{3}=-A_{P} \frac{r^{3}}{\mathrm{G}}
$$

When the spacetime expansion acceleration force is expressed by mass, it is expressed as $M_{3}$ of equation (7.27). From equation (7.27), if $A_{P}>0, M_{3}$ has a negative mass, and if $A_{P}<0$, $M_{3}$ has a positive mass.

Observations show that each proportion in total energy is expressed as [8]

$$
\text { The proportion of ordinary matter }=4.9 \% \text {, }
$$

The proportion of dark matter $=26.8 \%$,

The proportion of dark energy $=68.3 \%$.

We calculate the mass densities from each mass and proportion. In the following calculation, we calculate with $A_{P}\left(t=t_{1}\right)$ of the acceleration period matching condition.

From the proportion of $M_{1}$ and $M_{2}$, the mass relationship is expressed as

$$
\frac{\left|M_{1}\right|}{\left|M_{2}\right|}=\frac{4.9}{26.8}
$$

Substituting equations (7.25) and (7.26) into equation (7.31), it is expressed as

$$
r=\mathrm{r}_{\mathrm{e}} \times \frac{26.8}{4.9}
$$

We set the radius $r$ of equation (7.32) as $r_{A}$. From equations (3.24) and (7.32), the radius $r_{A}$ is calculated as

$$
r_{A}=2.92 \times 10^{19} \text { meters } .
$$

From equations (7.27) and (7.33), $M_{3}$ is expressed as

$$
M_{3}=-1.08 \times 10^{33} \mathrm{~kg} .
$$

Since each mass is in the sphere volume $V=\frac{4 \pi r_{A}^{3}}{3}$ with the radius $r_{A}$, the mass density $\frac{M_{3}}{V}$ of dark energy is expressed as 


$$
\frac{M_{3}}{V}=-1.03 \times 10^{-26} \mathrm{~kg} / \mathrm{m}^{3} .
$$

From the proportion, the mass and mass density of each are expressed as

$$
\begin{gathered}
M_{1}=7.76 \times 10^{31} \mathrm{~kg}, \\
\frac{M_{1}}{V}=7.42 \times 10^{-28} \mathrm{~kg} / \mathrm{m}^{3}, \\
M_{2}=4.24 \times 10^{32} \mathrm{~kg}, \\
\frac{M_{2}}{V}=4.06 \times 10^{-27} \mathrm{~kg} / \mathrm{m}^{3} .
\end{gathered}
$$

From equation (7.19), the distance $r_{e 2}$ where the sum of gravity ( $M_{1}$ and $M_{2}$ ) and the spacetime expansion acceleration force $\left(M_{3}\right)$ becomes zero is expressed as

$$
r_{e 2}=1.83 \times 10^{19} \text { meters } .
$$

We discuss the calculated results.

First, this calculation result means that if we average over the universe, the mass $M_{1}$ of ordinary matter, the mass $M_{2}$ of dark matter and the mass $M_{3}$ of dark energy exist for each sphere volume with the radius $r_{A}$.

Second, the relationship of $r_{A}>r_{e 2}$ shows that repulsive force acts between the sphere volumes with the radius $r_{A}$.

Third, the first and second conclusions mean that the entire universe expands at an accelerating rate in each sphere volume space unit by multiple expansion sources. The calculation of spacetime expansion in section 6 is based on this idea.

Fourth, $\frac{M_{1}}{V}, \frac{M_{2}}{V}$ and $\frac{M_{3}}{V}$ are mass densities when their respective masses are uniformly distributed in space, but only $\frac{M_{3}}{V}$ is actually uniformly distributed in space. Since $M_{3}=$ $-A_{P} \frac{r_{A}^{3}}{G}$, the mass density $\frac{M_{3}}{V}$ is expressed as

$$
\frac{M_{3}}{V}=-\frac{3 A_{P}}{4 \pi \mathrm{G}} .
$$

In summary, there are three forces received by mass: the gravity of ordinary matter, the gravity of spacetime particles, and the spacetime expansion acceleration force. The dominance of the three forces changes with distance, the gravity of ordinary matter is dominant at short-range, the gravity of spacetime particles is dominant at medium-range, and the spacetime expansion acceleration force is dominant at long-range. If we average over 
the universe, the mass $M_{1}$ of ordinary matter, the mass $M_{2}$ of dark matter and the mass $M_{3}$ of dark energy exist for each sphere volume with the radius $r_{A}$.

\section{Quantized Coordinate Transformations}

Spacetime particles composing spacetime move only at the speed of light, but spacetime expansion decelerates or accelerates. To solve this contradiction, in this section, we derive the quantized coordinate transformations. From the quantized coordinate transformations, we explain the expansion change of spacetime and the speed of wave function reduction.

\subsection{Derivation and Discussion of Quantized Coordinate Transformations}

We derive the quantized coordinate transformations. Then we discuss the quantized coordinate transformations.

First of all, we describe the problem of spacetime expansion. From equations (4.17) and (4.32), the spacetime particle number $N$ is expressed as

$$
N=\frac{t}{\Delta t}=\frac{M \mathrm{c} t}{\mathrm{k}_{\mathrm{sp}} T_{s p}}
$$

Substituting $N$ of equation (8.1) into the spacetime radius $r$ of equation (2.18), the spacetime radius $r$ is expressed as

$$
r=\mathrm{ct}
$$

Equation (8.2) means that gravitons carrying gravity move at the speed of light. This is a natural conclusion because time $t$ is defined on the premise of $r=$ ct. However, this causes spacetime to expand at a constant speed, which is not consistent with the observations of deceleration expansion and acceleration expansion.

From the calculation of spacetime expansion in section 6, we discuss this problem. In section 6, we assumed that the mass $M$ changes with time. From equation (4.17), the quantized minimum time $\Delta t$ also changes according to the change in mass. However, if we think so, the spacetime expands at a constant speed, and $\alpha 1$ in equation (6.3) is not a constant. Therefore, we conclude that observers on Earth observe the quantized minimum time $\Delta t$ as constant rather than time-varying. From this, the quantized minimum time $\Delta t$ is expressed as

$$
\Delta t=\frac{\mathrm{k}_{\mathrm{sp}} T_{s p}}{M \mathrm{c}}=\frac{1}{\alpha_{1}}
$$


where $\alpha 1$ is the constant in equation (6.3), the mass $M$ is a constant. The spacetime particle number $N$ is expressed as

$$
N=\frac{t}{\Delta t}=\alpha_{1} t
$$

Here, we set $N_{0}=0$ to match $N=0$ at $t=0$. We consider that the mass $M$ in the quantized minimum time $\Delta t$ is constant, but the mass $M$ in the spacetime radius $r$ changes with time as shown in equation (6.2). Then, the spacetime radius $r$ is expressed as

$$
r=\mathrm{k}_{\mathrm{sp}} T_{s p} \times \frac{\alpha_{1} t}{\beta_{2} t^{2}+\beta_{1} t+M_{0}} .
$$

Equation (8.5) is the same as the calculation in section 6 except that the origin coordinates of $t=0$ are different. Therefore, if we consider that the mass $M$ in $\Delta t$ and the mass $M$ in $r$ are different, we can match with the calculation in section 6 .

We discuss this problem from the relativity principle. The relativity principle is that if a phenomenon belongs to the same frame of reference as an observer, the physical law of the phenomenon can be described as the same form in that frame of reference. Conversely, if a phenomenon belongs to the frame of reference different from an observer, the physical law of the phenomenon can be described as the different form in that frame of reference.

The relativity principle suggests that the difference in mass $M$ is the difference in the frame of reference. First, the difference in the frame of reference is the difference in the quantized minimum time $\Delta t$ or the quantized minimum distance $\Delta r$. The spacetime radius $r$ is expressed as $r=N \Delta r$, and time $t$ is expressed as $t=N \Delta t$. If it is the same time, the spacetime particle number $N$ is all the same in any frame of reference. Therefore, we conclude that the difference in the frame of reference is the difference in the quantized minimum time $\Delta t$ or the quantized minimum distance $\Delta r$. Second, from $r=N \Delta r$ and $N=\frac{t}{\Delta t}$, as long as the reference frame of the quantized minimum time $\Delta t$ and the reference frame of the quantized minimum distance $\Delta r$ are different, there is no problem even if the mass $M$ is different. Therefore, from the relativity principle, we conclude that the difference in mass $M$ is the difference in the frame of reference.

From the above, we conclude as follows. If the mass $M$ in $\Delta t$ and the mass $M$ in $\Delta r$ are the same, the reference frames of $\Delta t$ and $\Delta r$ are the same, and the spacetime radius $r$ is $r=$ ct. If the mass $M$ in $\Delta t$ and the mass $M$ in $\Delta r$ are different, the references frame of $\Delta t$ and $\Delta r$ are different, and the spacetime radius $r$ is not $r=c t$. Therefore, the spacetime radius of the same reference frame as the observer expands at a constant speed of light, but the spacetime radius of the reference frame different from the observer does not expand at a constant speed of light.

From this conclusion, we derive the quantized coordinate transformations. We refer to the reference frame with a constant quantized minimum time as the $t$ frame and the 
reference frame with a time-varying quantized minimum time as the $t^{\prime}$ frame. From the above conclusion, if reference frames are the same, the spacetime radius must expand at a constant speed of light. Therefore, in the $t$ frame and the $t^{\prime}$ frame, the following equations hold.

$$
\begin{gathered}
t^{\prime}=N^{\prime} \Delta t^{\prime}, \\
t=N \Delta t,
\end{gathered}
$$

where $t^{\prime}, N^{\prime}$ and $\Delta t^{\prime}$ are the time, spacetime particle number and quantized minimum time of the $t^{\prime}$ frame, and $t, N$ and $\Delta t$ are the time, spacetime particle number and quantized minimum time of the $t$ frame.

$$
\begin{gathered}
\Delta t^{\prime}=\frac{\mathrm{k}_{\mathrm{sp}} T_{s p}^{\prime}}{M^{\prime} \mathrm{c}}, \\
\Delta t=\frac{\mathrm{k}_{\mathrm{sp}} T_{s p}}{M \mathrm{c}}=\frac{1}{\alpha_{1}},
\end{gathered}
$$

where $T_{s p}^{\prime}$ and $M^{\prime}$ are the dimensionless temperature and mass of the $t^{\prime}$ frame, and $T_{s p}$ and $M$ are the dimensionless temperature and mass of the $t$ frame.

$$
\begin{gathered}
N^{\prime}=\frac{t^{\prime}}{\Delta t^{\prime}}=\frac{M^{\prime} \mathrm{c}}{\mathrm{k}_{\mathrm{sp}} T_{s p}^{\prime}} \times t^{\prime}, \\
N=\frac{t}{\Delta t}=\frac{M \mathrm{c}}{\mathrm{k}_{\mathrm{sp}} T_{s p}} \times t=\alpha_{1} t, \\
r^{\prime}=\mathrm{c} t^{\prime}, \\
r=\mathrm{ct},
\end{gathered}
$$

where $r^{\prime}$ is the spacetime radius of the $t^{\prime}$ frame, and $r$ is the spacetime radius of the $t$ frame.

In both frames, the spacetime particle number at the same time is the same, so the following equation holds.

$$
N^{\prime}=N \text {. }
$$

From equations (8.10) and (8.11) and (8.14), the following equation holds.

$$
\frac{t^{\prime}}{t}=\frac{\Delta t^{\prime}}{\Delta t}=\mathrm{k}_{\mathrm{sp}} T_{s p}^{\prime} \times \frac{\alpha_{1}}{M^{\prime} \mathrm{c}} .
$$


From equation (8.15), $r^{\prime}$ is expressed as

$$
r^{\prime}=\mathrm{ct} t^{\prime}=\mathrm{k}_{\mathrm{sp}} T_{s p}^{\prime} \times \frac{\alpha_{1} t}{M^{\prime}} .
$$

Assuming that $T_{s p}=T_{s p}^{\prime}$ and $M^{\prime}=\beta_{2} t^{2}+\beta_{1} t+M_{0}$, equation (8.16) is consistent with equation (8.5). Therefore, we conclude that the spacetime radius $r$ of equation (8.5) is $r^{\prime}$ of equation (8.16) transformed by equation (8.15). Since the spacetime radius of equation (8.5) is the spacetime radius of the reference frame different from the observer, it does not expand at a constant speed of light.

From equation (8.9), $\alpha_{1}$ is expressed as

$$
\alpha_{1}=\frac{M \mathrm{c}}{\mathrm{k}_{\mathrm{sp}} T_{s p}} .
$$

From equation (8.17), equation (8.15) is expressed as

$$
\frac{t^{\prime}}{t}=\frac{\Delta t^{\prime}}{\Delta t}=\frac{T_{s p}^{\prime}}{T_{s p}} \times \frac{M}{M^{\prime}},
$$

and equation (8.16) is expressed as

$$
r^{\prime}=\mathrm{c} t^{\prime}=\mathrm{c} \times \frac{T_{s p}^{\prime}}{T_{s p}} \times \frac{M}{M^{\prime}} \times t
$$

From equations (4.3) and (8.12) and (8.13) and (8.19), the spacetime radius $r$ and $r^{\prime}$ are expressed as

$$
\begin{gathered}
r=N \Delta r=\mathrm{ct}, \\
r^{\prime}=N^{\prime} \Delta r^{\prime}=\mathrm{c} t^{\prime}=\mathrm{c} \times \frac{T_{s p}^{\prime}}{T_{s p}} \times \frac{M}{M^{\prime}} \times t .
\end{gathered}
$$

From equations (8.14) and (8.20) and (8.21), equation (8.18) is expressed as

$$
\frac{r^{\prime}}{r}=\frac{\Delta r^{\prime}}{\Delta r}=\frac{t^{\prime}}{t}=\frac{\Delta t^{\prime}}{\Delta t}=\frac{T_{s p}^{\prime}}{T_{s p}} \times \frac{M}{M^{\prime}} .
$$

We define $c^{\prime}$ as

$$
c^{\prime}=\mathrm{c} \times \frac{T_{s p}^{\prime}}{T_{s p}} \times \frac{M}{M^{\prime}} .
$$

Then, equation (8.22) is expressed as 


$$
\frac{r^{\prime}}{r}=\frac{\Delta r^{\prime}}{\Delta r}=\frac{t^{\prime}}{t}=\frac{\Delta t^{\prime}}{\Delta t}=\frac{c^{\prime}}{\mathrm{c}}=\frac{T_{s p}^{\prime}}{T_{s p}} \times \frac{M}{M^{\prime}} .
$$

From equation (8.24), the spacetime radius $r^{\prime}$ is expressed as

$$
r^{\prime}=c t^{\prime}=c^{\prime} t
$$

From equation (5.5), $T_{s p}$ is expressed as $T_{s p}=4 \pi s$, and $T_{s p}^{\prime}$ is expressed as $T_{s p}^{\prime}=4 \pi s^{\prime}$, where $s$ is the spin quantum number of the $t$ frame, and $s^{\prime}$ is the spin quantum number of the $t^{\prime}$ frame. From these, equation (8.24) is expressed as

$$
\frac{r^{\prime}}{r}=\frac{\Delta r^{\prime}}{\Delta r}=\frac{t^{\prime}}{t}=\frac{\Delta t^{\prime}}{\Delta t}=\frac{c^{\prime}}{\mathrm{c}}=\frac{s^{\prime}}{s} \times \frac{M}{M^{\prime}} .
$$

From equations (4.35) and (5.11), $M$ and $M^{\prime}$ are expressed as

$$
\begin{gathered}
M=n_{\max } \times \mathrm{m}_{\mathrm{sp} 0} \times 4 \pi s, \\
M^{\prime}=n_{\text {max }}^{\prime} \times \mathrm{m}_{\mathrm{sp} 0} \times 4 \pi s^{\prime},
\end{gathered}
$$

where $n_{\max }$ is the total spacetime particle number within the matter of the $t$ frame, and $n_{\max }^{\prime}$ is the total spacetime particle number within the matter of the $t^{\prime}$ frame. From equations (8.27) and (8.28), equation (8.26) is expressed as

$$
\frac{r^{\prime}}{r}=\frac{\Delta r^{\prime}}{\Delta r}=\frac{t^{\prime}}{t}=\frac{\Delta t^{\prime}}{\Delta t}=\frac{c^{\prime}}{\mathrm{c}}=\frac{s^{\prime}}{s} \times \frac{M}{M^{\prime}}=\frac{n_{\max }}{n_{\max }^{\prime}} .
$$

Finally, $\frac{r^{\prime}}{r}=\frac{\Delta r^{\prime}}{\Delta r}=\frac{t^{\prime}}{t}=\frac{\Delta t^{\prime}}{\Delta t}$ is expressed as an integer ratio of the spacetime particle number within the matter. Equations (8.26) and (8.29) are coordinate transformations based on the quantized minimum time and the quantized minimum distance. Therefore, the coordinate transformations of equations (8.26) and (8.29) are the quantized coordinate transformations. In this way, the quantized coordinate transformations are derived.

We can also derive the quantized coordinate transformation from the spin angular momentum for the central matter. From equation (5.3), the spin angular momentum of an elementary particle of the $t$ frame is expressed as

$$
\hbar s=M c r_{0},
$$

where $s, M$ and $r_{0}$ are the spin quantum number, mass and radius of an elementary particle of the $t$ frame. The spin angular momentum of an elementary particle of the $t^{\prime}$ frame is expressed as 


$$
\hbar s^{\prime}=M^{\prime} c r_{0}^{\prime}
$$

where $s^{\prime}, M^{\prime}$ and $r_{0}^{\prime}$ are the spin quantum number, mass and radius of an elementary particle of the $t^{\prime}$ frame. When we rearrange equations (8.30) and (8.31), they are expressed as

$$
\begin{aligned}
& \frac{\hbar}{\mathrm{c}}=\frac{M r_{0}}{s}, \\
& \frac{\hbar}{\mathrm{c}}=\frac{M^{\prime} r_{0}^{\prime}}{s^{\prime}} .
\end{aligned}
$$

Since $\frac{\hbar}{\mathrm{c}}$ is a constant, the following equation holds between different reference frames.

$$
\frac{M r_{0}}{s}=\frac{M^{\prime} r_{0}^{\prime}}{s^{\prime}}
$$

When we rearrange equation (8.34), it is expressed as

$$
\frac{r_{0}^{\prime}}{r_{0}}=\frac{s^{\prime}}{s} \times \frac{M}{M^{\prime}}
$$

From $r_{0}=\frac{\Delta r}{2}$ and $r_{0}^{\prime}=\frac{\Delta r^{\prime}}{2}$ and so on, equation (8.35) is expressed as

$$
\frac{r^{\prime}}{r}=\frac{\Delta r^{\prime}}{\Delta r}=\frac{t^{\prime}}{t}=\frac{\Delta t^{\prime}}{\Delta t}=\frac{s^{\prime}}{s} \times \frac{M}{M^{\prime}} .
$$

If we do not delete the rotational speed $c$ of the elementary particle, equation (8.35) is expressed as $\mathrm{c} \times \frac{r_{0}^{\prime}}{r_{0}}=\mathrm{c} \times \frac{s^{\prime}}{s} \times \frac{M}{M^{\prime}}$. When we define $c^{\prime}$ as $c^{\prime}=\mathrm{c} \times \frac{s^{\prime}}{s} \times \frac{M}{M^{\prime}}$, equation (8.36) is expressed as

$$
\frac{r^{\prime}}{r}=\frac{\Delta r^{\prime}}{\Delta r}=\frac{t^{\prime}}{t}=\frac{\Delta t^{\prime}}{\Delta t}=\frac{c^{\prime}}{\mathrm{c}}=\frac{s^{\prime}}{s} \times \frac{M}{M^{\prime}}
$$

Equation (8.37) is the same as equation (8.26), and it is the quantized coordinate transformations. In this way, we can also derive the quantized coordinate transformations from the spin angular momentum for the central matter.

We discuss the physical meanings of the light speeds $\mathrm{c}$ and $c^{\prime}$ in equations (8.26) and (8.37). The light speeds $c$ and $c^{\prime}$ in equation (8.37) are the rotational speed of spacetime particles within an elementary particle. The light speeds $c$ and $c^{\prime}$ in equation (8.26) are the speed of the radius of spacetime particles around an elementary particle or matter. At even physically different speeds, the same quantized coordinate transformations hold. From this, if a reference frame is the same, the following equation holds.

$$
v_{\text {rot }}=v_{\text {rad }}
$$


where $v_{r o t}$ is the rotational velocity of spacetime particles, $v_{\text {rad }}$ is the velocity of the radius of spacetime particles, and reference frames of $v_{\text {rot }}$ and $v_{\text {rad }}$ are the same.

Equation (8.38) suggests that the rotational velocity of spacetime particles and the velocity of the radius of spacetime particles are physically equivalent. First, both velocities of equation (8.38) are the velocities of spacetime particles. Second, at both velocities, the center coordinate of spacetime particles does not change. Third, kinetic energy must be conserved between when the spacetime particle is inside the elementary particle and when it is outside the elementary particle. From this, even if the radius of spacetime particles moves, its kinetic energy must be the same as rotational kinetic energy. Therefore, the velocities must be the same because the masses are the same. Fourth, at even physically different speeds, the same quantized coordinate transformations hold. Fifth, from the minimum space properties, if the motion is within the minimum space of graviton coordinates, the rotation direction and the radial direction are equivalent. Sixth, from the complementarity principle, a complete description can be made by considering that mutually contradictory elements are realized simultaneously or are the same. Therefore, we conclude that the rotational velocity of spacetime particles and the velocity of the radius of spacetime particles are physically equivalent. That is, when the radius of the spacetime particle is moving at the velocity c, it is physically equivalent to the spacetime particle rotating at the velocity $\mathrm{c}$ at the same time. Conversely, when the spacetime particle is rotating at the velocity c, it is physically equivalent to the radius of the spacetime particle moving at the velocity $\mathrm{c}$ at the same time.

We compare the quantized coordinate transformations and the Lorentz transformations. From the theory of relativity, the mass $M^{\prime}$ of an object moving at the translational velocity $v$ is expressed as

$$
M^{\prime}=\frac{M}{\sqrt{1-\left(\frac{v}{c}\right)^{2}}},
$$

where $M$ is the rest mass of the object. The Lorentz transformations and the quantized coordinate transformations are coordinate transformations derived from the same relativity principle. Therefore, if the spin quantum numbers $s$ and $s^{\prime}$ are the same, from equation (8.39), equation (8.26) is expressed as

$$
\frac{r^{\prime}}{r}=\frac{\Delta r^{\prime}}{\Delta r}=\frac{t^{\prime}}{t}=\frac{\Delta t^{\prime}}{\Delta t}=\frac{c^{\prime}}{\mathrm{c}}=\frac{M}{M^{\prime}}=\sqrt{1-\left(\frac{v}{\mathrm{c}}\right)^{2}} .
$$

In this way, the Lorentz transformations and the quantized coordinate transformations are highly similar and easy to compare. Therefore, we show the difference between the quantized coordinate transformations and the Lorentz transformations.

First, the quantized coordinate transformations depend on the spin quantum number, but the Lorentz transformations do not depend on the spin quantum number. 
Second, since $r, r^{\prime}, \Delta r$ and $\Delta r^{\prime}$ are spacetime radii, the quantized coordinate transformations hold only in the quantized graviton coordinates, but the Lorentz transformations hold in the continuous real coordinates. Therefore, in this paper, the quantized coordinate transformations are applied only in the minimum space unit of graviton coordinates. For example, within the elementary particle radius of $r_{0}=\frac{\Delta r}{2}$, the entire space is one minimum space, and the quantized coordinate transformations are applied to the entire space.

Third, the light speeds $c$ and $c^{\prime}$ in the quantized coordinate transformations are the rotational velocity of spacetime particles or the velocity of the radius of spacetime particles, but the light speed $\mathrm{c}$ in the Lorentz transformations are the translational velocity of photons. Even at the same light speed, the physical meanings of the speeds are different.

Fourth, the quantized coordinate transformations are derived from spacetime particles composing spacetime, but the Lorentz transformations are derived from photons that are not directly related to spacetime. However, since photons have no charge, they are composed only of spacetime particles.

There is such a difference between the quantized coordinate transformations and the Lorentz transformations. From such a difference, in section 16, the Lorentz transformations of this paper are derived as a form different from the Lorentz transformations of relativity.

We discuss the physical quantities observed from the different reference frames. From the discussion of the quantized coordinate transformations, we conclude as follows. The spacetime radius $r^{\prime}$ of the $t^{\prime}$ frame observed from the $t^{\prime}$ frame is expressed as $r^{\prime}=c t^{\prime}$. The spacetime radius $r^{\prime}$ of the $t^{\prime}$ frame observed from the $t$ frame is expressed as $r^{\prime}=\mathrm{c}^{\prime} t$.

That is, the speed of light in the different reference frames is observed as $c^{\prime}$, and is not observed as a constant light speed c. Similarly, all physical quantities in the different reference frames are observed as changing. As for the physical quantity that is specifically observed, the physical quantity of the $t^{\prime}$ frame is transformed into the $t$ frame by the quantized coordinate conversion, and the physical quantity calculated by the time and distance of the $t$ frame is actually observed.

As an example, the kinetic energy of special relativity is calculated from equation (8.40) as follows.

$$
\begin{gathered}
p^{\prime}=m^{\prime} v=\frac{m v}{\sqrt{1-\left(\frac{v}{\mathrm{c}}\right)^{2}}}, \\
F^{\prime}=\frac{\mathrm{d} p^{\prime}}{\mathrm{d} t}=\frac{m \frac{\mathrm{d} v}{\mathrm{~d} t}}{\left(1-\left(\frac{v}{\mathrm{c}}\right)^{2}\right)^{3 / 2}}, \\
W^{\prime}=\int F^{\prime} \mathrm{d} r=\frac{m \mathrm{c}^{2}}{\sqrt{1-\left(\frac{v}{\mathrm{c}}\right)^{2}}}-m \mathrm{c}^{2},
\end{gathered}
$$

where $p^{\prime}$ is the momentum of an object, $F^{\prime}$ is the force exerted on the object, $W^{\prime}$ is is the work required to change the object from velocity 0 to velocity $v$, they are all the physical 
quantities of the $t^{\prime}$ frame observed from the $t$ frame, the $t^{\prime}$ frame is the reference frame that moves at a translational velocity $v$, the $t$ frame is the rest frame. From equation (8.43), the kinetic energy at velocity $v$ is expressed as $\frac{m c^{2}}{\sqrt{1-\left(\frac{v}{c}\right)^{2}}}$.

The reason why we can calculate in this way can be explained as follows. The physical quantities of the $t^{\prime}$ frame are the physical quantities observed by the observer of the $t^{\prime}$ frame. The physical quantities of the $t$ frame are the physical quantities observed by the observer of the $t$ frame. For this reason, when calculated with the physical quantity of the $t$ frame, it becomes the physical quantity observed by the observer of the $t$ frame. When calculated with the physical quantity of the $t^{\prime}$ frame, it becomes the physical quantity observed by the observer of the $t^{\prime}$ frame. Therefore, the physical quantity of the $t^{\prime}$ frame is transformed into the $t$ frame, and the physical quantity calculated by the time and distance of the $t$ frame becomes the physical quantity of the $t^{\prime}$ frame observed by the observer of the $t$ frame. In this paper, we calculate the physical quantities of different reference frames based on this idea.

We discuss the variable transformations in differentiation based on the quantized coordinate transformations. The limit to 0 in differentiation is expressed as $\mathrm{d} t=\Delta t, \mathrm{~d} t^{\prime}=\Delta t^{\prime}, \mathrm{d} r=\Delta r$, $\mathrm{d} r^{\prime}=\Delta r^{\prime}$. Therefore, from equation (8.26), the variable transformations in differentiation based on the quantized coordinate transformations are expressed as

$$
\frac{\mathrm{d} t^{\prime}}{\mathrm{d} t}=\frac{\mathrm{d} r^{\prime}}{\mathrm{d} r}=\frac{c^{\prime}}{\mathrm{c}}=\frac{s^{\prime}}{s} \times \frac{M}{M^{\prime}}
$$

As an example, we consider the differentiation $\frac{\mathrm{d} r^{\prime}}{\mathrm{d} t}$ of $r^{\prime}=c^{\prime} t(r=\mathrm{c} t)$. In ordinary differentiation, $\frac{\mathrm{d} r^{\prime}}{\mathrm{d} t}$ is expressed as

$$
\frac{\mathrm{d} r^{\prime}}{\mathrm{d} t}=c^{\prime}+t \frac{\mathrm{d} c^{\prime}}{\mathrm{d} t}
$$

However, if $r^{\prime}=c t^{\prime}\left(=c^{\prime} t\right), \frac{\mathrm{d} r^{\prime}}{\mathrm{d} t}$ is expressed as $\frac{\mathrm{d} r^{\prime}}{\mathrm{d} t}=\mathrm{c} \frac{\mathrm{d} t^{\prime}}{\mathrm{d} t}=\mathrm{c} \times \frac{c^{\prime}}{\mathrm{c}}=c^{\prime}$, so equation (8.45) is a mistake. In the differentiation based on the quantized coordinate transformations, from $\mathrm{d} r^{\prime}=\frac{c^{\prime}}{\mathrm{c}} \times \mathrm{d} r$ and $\frac{\mathrm{d} r}{\mathrm{~d} t}=\mathrm{c}, \frac{\mathrm{d} r^{\prime}}{\mathrm{d} t}$ is expressed as

$$
\frac{\mathrm{d} r^{\prime}}{\mathrm{d} t}=\frac{c^{\prime}}{\mathrm{c}} \times \frac{\mathrm{d} r}{\mathrm{~d} t}=c^{\prime}
$$

Therefore, the differentiation $\frac{\mathrm{d} r^{\prime}}{\mathrm{d} t}$ of $r^{\prime}=c^{\prime} t$ is $c^{\prime}$.

\subsection{Change in Spacetime Expansion Based on the Quantized Coordinate Transformations}

From the quantized coordinate transformations, we discuss the spacetime expansion that belongs to the reference frames different from the observer, and we confirm that the discussion is consistent with sections 6 and 7 . 
Here, as in section 6 , we set the spacetime radius as $r_{\max }$ and the inter-matter radius as $r$. We refer to the reference frame with a constant quantized minimum time as the $t$ frame and the reference frame with a time-varying quantized minimum time as the $t^{\prime}$ frame.

From equation (8.25), the spacetime radius $r_{\max }^{\prime}$ of $t^{\prime}$ frame is expressed as

$$
r_{\text {max }}^{\prime}=\mathrm{ct}^{\prime}=c^{\prime} t
$$

In spacetime expansion, only the long-range of $r_{e 2}<<r$ is considered, so gravity can be ignored. Moreover, if the inter-matter radius changes by the gravity of other matter, that change is not a spacetime expansion, but just a change in position. From these, gravity is not considered in spacetime expansion. Then, the inter-matter radius changes in the same way as the spacetime radius, and the inter-matter radius $r^{\prime}$ of the $t^{\prime}$ frame is expressed as

$$
r^{\prime}=v t^{\prime}=v^{\prime} t
$$

where $v$ is the expansion speed of the inter-matter radius of the $t^{\prime}$ frame observed from the $t^{\prime}$ frame, $v^{\prime}$ is the expansion speed of the inter-matter radius of the $t^{\prime}$ frame observed from the $t$ frame. Similar to the expansion speed of the spacetime radius, $v$ is a constant, and $v^{\prime}$ is a variable.

From equations (8.47) and (8.48), $\frac{r^{\prime}}{r_{\max }^{\prime}}$ is expressed as

$$
\frac{r^{\prime}}{r_{\max }^{\prime}}=\frac{v^{\prime} t}{c^{\prime} t}=\frac{v^{\prime}}{c^{\prime}}
$$

From equation (8.46), the expansion speed $v_{\text {max }}^{\prime}$ of the spacetime radius of the $t^{\prime}$ frame observed from the $t$ frame is expressed as

$$
v_{\text {max }}^{\prime}=\frac{\mathrm{d} r_{\max }^{\prime}}{\mathrm{d} t}=c^{\prime}
$$

From equation (8.50), equation (8.49) is expressed as

$$
\frac{r^{\prime}}{r_{\max }^{\prime}}=\frac{v^{\prime}}{v_{\max }^{\prime}}
$$

Equation (8.51) is consistent with equation (6.4). From equation (8.51), $v^{\prime}$ and the Hubble parameter $H$ are expressed as

$$
\begin{gathered}
v^{\prime}=r^{\prime} \times \frac{v_{\max }^{\prime}}{r_{\max }^{\prime}}, \\
H=\frac{v_{\max }^{\prime}}{r_{\max }^{\prime}},
\end{gathered}
$$




$$
v^{\prime}=r^{\prime} H
$$

From equation (8.15), the spacetime radius $r_{\max }^{\prime}$ is expressed as

$$
r_{\text {max }}^{\prime}=\mathrm{ct}^{\prime}=\mathrm{k}_{\mathrm{sp}} T_{s p}^{\prime} \times \frac{\alpha_{1} t}{M^{\prime}}
$$

By changing the origin coordinates, we change $\alpha_{1} t$ to $N_{0}\left(a_{1} t+1\right)$, and we assume $M^{\prime}$ and $T_{s p}^{\prime}$ as

$$
\begin{gathered}
M^{\prime}=M_{0}\left(b_{2} t^{2}+b_{1} t+1\right), \\
T_{s p}^{\prime}=T_{s p} .
\end{gathered}
$$

Then, various parameters are expressed as follows.

$$
\begin{gathered}
r_{\text {max }}^{\prime}=\mathrm{k}_{\mathrm{sp}} T_{s p} \times \frac{N_{0}}{M_{0}} \times \frac{a_{1} t+1}{b_{2} t^{2}+b_{1} t+1}, \\
r_{\text {max }}^{\prime}(t=0)=\mathrm{k}_{\mathrm{sp}} T_{s p} \times \frac{N_{0}}{M_{0}}, \\
E_{R}=\frac{r_{\text {max }}^{\prime}(t)}{r_{\text {max }}^{\prime}(t=0)}=\frac{a_{1} t+1}{b_{2} t^{2}+b_{1} t+1}, \\
r_{\text {max }}^{\prime}=r_{\text {max }}^{\prime}(t=0) \times E_{R}, \\
v_{\text {max }}^{\prime}=r_{\text {max }}^{\prime}(t=0) \times \frac{\mathrm{d} E_{R}}{\mathrm{~d} t}, \\
a_{\text {max }}^{\prime}=r_{\text {max }}^{\prime}(t=0) \times \frac{\mathrm{d}^{2} E_{R}}{\mathrm{~d} t^{2}} \\
H=\frac{v_{\text {max }}^{\prime}}{r_{\text {max }}^{\prime}}=\frac{\left(\frac{\mathrm{d} E_{R}}{\mathrm{~d} t}\right)}{E_{R}}, \\
A_{P}=\frac{a_{\text {max }}^{\prime}}{r_{\text {max }}^{\prime}}=\frac{\left(\frac{\mathrm{d}^{2} E_{R}}{\mathrm{~d} t^{2}}\right)}{E_{R}}
\end{gathered}
$$

where $a_{\text {max }}^{\prime}$ is the expansion acceleration of the spacetime radius of $t^{\prime}$ frame observed from the $t$ frame.

When $t^{\prime}$ of $r^{\prime}=v t^{\prime}$ is transformed to $t$ by equation (8.15), it is expressed as 


$$
r^{\prime}=v t^{\prime}=\mathrm{k}_{\mathrm{sp}} T_{s p}^{\prime} \times \frac{\alpha_{1} t}{M^{\prime}} \times \frac{v}{\mathrm{c}} .
$$

From equations (8.55) and (8.61), equation (8.66) is expressed as

$$
r^{\prime}=v t^{\prime}=r_{\max }^{\prime} \times \frac{v}{\mathrm{c}}=r_{\max }^{\prime}(t=0) \times \frac{v}{\mathrm{c}} \times E_{R} .
$$

We set a part of equation (8.67) as

$$
r^{\prime}(t=0)=r_{\max }^{\prime}(t=0) \times \frac{v}{\mathrm{c}} .
$$

Since $v$ is a constant, $r^{\prime}(t=0)$ is a constant. From equations (8.67) and (8.68), $r^{\prime}, v^{\prime}$ and the expansion acceleration $a^{\prime}$ of the inter-matter radius of $t^{\prime}$ frame observed from the $t$ frame are expressed as

$$
\begin{gathered}
r^{\prime}=r^{\prime}(t=0) \times E_{R}, \\
v^{\prime}=r^{\prime}(t=0) \times \frac{\mathrm{d} E_{R}}{\mathrm{~d} t}=r^{\prime} H, \\
a^{\prime}=r^{\prime}(t=0) \times \frac{\mathrm{d}^{2} E_{R}}{\mathrm{~d} t^{2}}=r^{\prime} A_{P} .
\end{gathered}
$$

Therefore, these equations are consistent with the equations in sections 6 and 7 .

\subsection{Wave Function Reduction}

From the quantized coordinate transformations, we discuss the speed of wave function reduction. Wave function reduction in this paper is a phenomenon in which a spatially spread wave function contracts to one point at superluminal speed when observed. The speed of wave function reduction is the speed at which the wave function contracts to one point.

First of all, we show assumptions to explain the speed of wave function reduction.

Assumption 8.1: The square of the absolute value of a wave function represents the spacetime particle number density within an elementary particle, and it is expressed as

$$
n_{\max }|\psi|^{2}=\frac{\mathrm{d} n}{\mathrm{~d} V}
$$

where $\psi$ is the wave function, $V$ is any volume within the elementary particle, $n$ is the spacetime particle number contained in the volume $V$, and $\frac{\mathrm{d} n}{\mathrm{~d} V}$ and $n_{\max }$ are the spacetime particle number density and total spacetime particle number within the elementary particle. 
First, from the quantum principle, every element can be quantized by the smallest element and can be described based on particles.

Second, from assumption 5.1, an elementary particle is composed of spacetime particles with energy and charged particles with charges.

Third, the wave function is a function that describes an elementary particle, that is, spacetime particles and charged particles within the elementary particle.

Fourth, the wave function includes elements of momentum $p$ and energy $E$, but does not include charges. Moreover, equations based on the wave function such as the Schrödinger equation are derived from the energy of an elementary particle. That is, the wave function is related to energy, not charges.

Fifth, the square of the absolute value of the wave function has a unit of $1 / \mathrm{m}^{3}$. That is, its unit is the dimensionless number density. Moreover, the particles that compose the volume within an elementary particle are spacetime particles, and the particles that exist always in that volume are only spacetime particles.

Sixth, integrating the square of the absolute value of the wave function over the entire space yields 1. That is, the wave function is an element that can be added or divided, and when the wave function of the entire space is added together, they constitute a single elementary particle. The minimum unit of charge is one elementary particle. The minimum unit of energy or mass is one spacetime particle, and there are a huge number of spacetime particles within an elementary particle.

Seventh, assuming that equation (8.72) holds even when observed, from equations (5.13) and (5.16), in that case, the square of the absolute value of the wave function is expressed as

$$
|\psi|^{2}=\frac{1}{4 \pi r^{2} \times r_{0}}\left(\Delta r_{i n} \leqq r \leqq r_{0}=\frac{\Delta r}{2}\right) .
$$

Since $\Delta r_{i n}$ is extremely small, the graph of equation (8.73) in the range of $\Delta r_{i n} \leqq r \leqq r_{0}$ becomes a graph like the Dirac delta function.

Eighth, the particles in an elementary particle that can be actually observed are only charged particles, and charged particles at the time when observed exist in the center of the elementary particle where the spacetime particle number density is the highest. That is, elementary particles are observed in the space with the highest spacetime particle number density.

From the above, we conclude assumption 8.1.

Assumption 8.1 leads us to conclude the following. A wave function is a function for a huge number of spacetime particles within an elementary particle. Since spacetime particles are harmonic oscillators, the Schrödinger equation and so on are equations that arise by the huge number of harmonic oscillators. Although the motion of an elementary particle can be known only stochastically, that reason is that there are a huge number of spacetime particles that compose the elementary particle. Assuming that the observation of an elementary particle 
is proportional to the spacetime particle number density, we can interpret the spacetime particle number density within elementary particles as the probability density.

Assumption 8.2: Wave function reduction is the following phenomenon. Before observation, the spacetime particle distribution within an elementary particle expands to the minimum radius $\frac{\Delta r}{2}$ or more. This state has wave characteristics because the elementary particle radius changes flexibly. When observed, that distribution contracts to the minimum radius $\frac{\Delta r}{2}$ around the observation point. This state has particle characteristics because the elementary particle radius is fixed. From the minimum space properties, even the observation of one point within the elementary particle is equivalent to the observation in contact with the entire elementary particle, and the elementary particle is affected by such observation.

From assumption 8.1, the space where a wave function is distributed is the space where spacetime particles within an elementary particle are distributed. From equation (5.6), the spacetime particle radius of the elementary particle at the time of observation is $\frac{\Delta r}{2}$. Therefore, we conclude assumption 8.2.

Assumption 8.3: When wave function reduction occurs, only the radius of an elementary particle changes at the speed of light, the position of the elementary particle does not change, and the translational velocity of the elementary particle is zero.

First, the elementary particle radius is the radius where spacetime particles within the elementary particle are distributed.

Second, since there are no gravitons within the elementary particle radius, an inertial force does not work and an object moves at the speed of light.

Third, from equation (8.38), the rotational speed of spacetime particles is equivalent to the speed of the radius of spacetime particles, and the rotational speed of spacetime particles is the speed of light.

Fourth, in the change of radius, the position of an elementary particle does not change. That is, the translational velocity is zero. From the minimum space properties, the space within the elementary particle radius is one minimum space. For this reason, we can consider that the position of the elementary particle is located anywhere in that space. Therefore, even if the radius contracts around any point within the elementary particle radius, the position of the elementary particle does not change.

Therefore, we conclude assumption 8.3.

From assumption 8.3, the change $r^{\prime}$ of the elementary particle radius is expressed as

$$
r^{\prime}=c t^{\prime}
$$

where $t^{\prime}$ is the time within the elementary particle.

Assumption 8.4: The time $t^{\prime}$ within the elementary particle is based on the mass $M^{\prime}$ and $T_{s p}^{\prime}$ of the elementary particle, unlike the time $t$ of the observer on Earth. The observer's time $t$ on Earth is based on the mass $M$ and $T_{s p}$ of Earth. 
First, wave function reduction is a phenomenon faster than the speed of light. From assumption 8.3, when wave function reduction occurs, the elementary particle radius changes at the speed of light. From the relativity principle, when the phenomenon of the speed of light is observed at a speed other than the speed of light, the phenomenon belongs to a reference frame different from the observer. Second, wave function reduction occurs only in the elementary particle that is observed. Third, the greatest gravity that an observer on Earth receives is the gravity of Earth. Therefore, we assume assumption 8.4.

From these assumptions and the quantized coordinate transformations, we calculate the speed of wave function reduction. From equation (8.26), the change $r^{\prime}$ of the elementary particle radius is expressed as

$$
r^{\prime}=\mathrm{c} t^{\prime}=\mathrm{c} \times \frac{s^{\prime}}{s} \times \frac{M}{M^{\prime}} \times t
$$

where $t$ is the time of the observer on Earth, $M^{\prime}, T_{s p}^{\prime}$ and $s^{\prime}$ are the mass, dimensionless temperature and spin quantum number of the elementary particle, $M, T_{s p}$ and $s$ are the mass, dimensionless temperature and spin quantum number of Earth.

As an example, we calculate the case of electrons. The electron mass is $9.1093837015 \times$ $10^{-31} \mathrm{~kg}$ (2018 CODATA value), and the spin quantum number of electrons is $1 / 2$. The mass of Earth is $5.9722 \times 10^{24} \mathrm{~kg}$ (the recommended value published by the International Astronomical Union in 2009), and we assume that the spin quantum number of Earth is $1 / 2$. Therefore, the change $r^{\prime}$ of the elementary particle radius is expressed as

$$
r^{\prime}=\mathrm{c} \times 6.5561 \times 10^{54} \times t .
$$

The light speed $c^{\prime}$ of the electron radius is expressed as

$$
c^{\prime}=\mathrm{c} \times 6.5561 \times 10^{54} .
$$

The time $t^{\prime}$ within the electron is expressed as

$$
t^{\prime}=t \times 6.5561 \times 10^{54} .
$$

From equation (8.78), we observe that the time within the electron is $6.5561 \times 10^{54}$ times faster than the time of the observer on Earth. Alternatively, from equations (8.77) and (8.76), we observe that the electron radius is changing at the speed of $6.5561 \times 10^{54}$ times the speed of light. This changes at $6.6426 \times 10^{-38}$ seconds even at a distance of 13.8 billion light-years. Therefore, we observe that the electron wave function contracts in almost zero seconds. This is why a wave function contracts in almost zero seconds.

The summary is as follows. The square of the absolute value of a wave function represents the spacetime particle number density within an elementary particle. For this reason, wave function reduction is a phenomenon in which the spacetime particle radius of an elementary 
particle expands to the minimum radius $\frac{\Delta r}{2}$ or more before observation, but contracts to the minimum radius $\frac{\Delta r}{2}$ when observed. When observed from within the elementary particle, the wave function or the spacetime particle radius contracts at the light speed c. However, when observed from an observer on Earth, we observe that the wave function or the spacetime particle radius contracts at superluminal speed $c^{\prime}$ because time progresses differently.

In this way, based on the relativity principle, the quantized coordinate transformations are derived from the equation of state of spacetime particles or the spin angular momentum. The quantized coordinate transformations explain the spacetime expansion change and the speed of wave function reduction.

\section{Spacetime Change and Kinetic Energy}

In this section, from the law of energy conservation between different reference frames and the quantized coordinate transformations, we derive the spacetime change caused by translational velocity and gravity, and we derive kinetic energy at each spacetime change. The spacetime change in this paper means that space contracts or expands and time advances slower or faster. In section 8, the quantized coordinate transformations were derived from constant physical quantity between different reference frames and the relativity principle. Similarly, the spacetime change and the kinetic energy are derived from the law of energy conservation between different reference frames. That is, the spacetime change is caused based on constant physical quantity between different reference frames.

\subsection{Energy and Velocity of Particles Without Active Gravitational Mass}

We explain the energy and velocity of particles without active gravitational mass. The energy of all particles is composed of spacetime particles with energy. That is, the energies of all particles must be able to be calculated based on spacetime particles. Spacetime particles are particles without active gravitational mass. Therefore, to consider kinetic energy, it is necessary to consider the energy and velocity of particles without active gravitational mass.

First of all, we explain the energy of particles without active gravitational mass. In this paper, from assumption 2.10, particles without active gravitational mass have inertial mass and passive gravitational mass. For this reason, particles without active gravitational mass have kinetic energy by inertial mass and potential energy of gravity by passive gravitational mass. They are assumed as follows.

Assumption 9.1: The kinetic energy $E$ of particles without active gravitational mass is expressed as 


$$
E=m v^{2}
$$

where $m$ and $v$ are the inertial mass and speed of particles without active gravitational mass. The potential energy $U$ of gravity of particles without active gravitational mass is expressed as

$$
U=-\frac{2 \mathrm{G} M m}{r},
$$

where $m$ is the passive gravitational mass of particles without active gravitational mass, $M$ is the active gravitational mass of an external object that exerts gravity.

Since assumption 9.1 is an assumption for particles without active gravitational mass, it can be applied not only to spacetime particles but also to photons and so on.

We explain the velocity of particles without active gravitational mass. As an example of particles without active gravitational mass, we consider spacetime particles composing spacetime. The radius of spacetime particles composing spacetime changes at the speed of light. Moreover, spacetime particles composing spacetime translate in conjunction with the translational motion of the central matter. For this reason, the translational velocity of the central matter is the translational velocity of spacetime particles composing spacetime. From equation (8.38), the rotational velocity of spacetime particles and the velocity of the radius of that are physically equivalent. Therefore, spacetime particles composing spacetime have translational speed and rotational speed (= the velocity of the radius).

All particles without active gravitational mass must have the same properties as spacetime particles composing spacetime. First, focusing only on energy, all particles are groups of spacetime particles. Therefore, the energies of all particles must be able to be calculated based on spacetime particles. Second, like all particles without active gravitational mass, spacetime particles composing spacetime move at the speed of light. Therefore, we conclude that all particles without active gravitational mass have the same properties as spacetime particles composing spacetime. For this reason, all particles without active gravitational mass have the following properties.

Properties of particles without active gravitational mass:

First, particles without active gravitational mass belong to the spacetime of matter with active gravitational mass. This means that particles without active gravitational mass are affected by the spacetime of matter to which they belong.

Second, particles without active gravitational mass translate in conjunction with the translational motion of matter to which they belong. For this reason, the translational velocity of the matter to which they belong is the translational velocity of particles without active gravitational mass.

Third, the radius of particles without active gravitational mass changes at the speed of light. 
Fourth, from wave function reduction, particles without active gravitational mass, except for spacetime particles composing spacetime, have the following characteristics. Before observation, its elementary particle radius expands to the minimum radius $\frac{\Delta r}{2}$ or more. This state has wave characteristics because the elementary particle radius changes flexibly. When observed, its elementary particle radius contracts to the minimum radius $\frac{\Delta r}{2}$ around the observation point at superluminal speed. This state has particle characteristics because the elementary particle radius is fixed. From the minimum space properties, even the observation of one point within the elementary particle is equivalent to the observation in contact with the entire elementary particle, and the elementary particle is affected by such observation. Owing to these characteristics, the change in radius at the speed of light is equivalent to the change in position at the speed of light. However, if it belongs to the matter of the rest frame, its translational velocity is zero. From the minimum space properties, the space within the radius of the elementary particle is one minimum space. For this reason, we can consider that the position of the elementary particle is located anywhere in that space. Therefore, even if the elementary particle radius contracts around any point within the elementary particle radius, the position of the elementary particle does not change. That is, in wave function reduction, the translational velocity is zero.

Fifth, from equation (8.38), the rotational velocity of particles without active gravitational mass and the velocity of the radius of that are physically equivalent.

Particles without active gravitational mass have these properties.

\subsection{Law of Energy Conservation Between Different Reference Frames}

We derive the law of energy conservation between different reference frames.

First of all, we show assumptions and premise for that.

The individual elements in this section have the following meanings: The $t$ frame is the rest frame located at infinity from the source of gravity. The $t^{\prime}$ frame is the frame located at the radius $r$ from the source of gravity and the frame moving at a translational velocity $v^{\prime}$ against the $t$ frame. The speed of light $c^{\prime}$ is the speed of the radius of particles without active gravitational mass or the rotational speed of that, which is the light speed of the $t^{\prime}$ frame observed from the $t$ frame. The velocity $v^{\prime}$ is the translational velocity of the $t^{\prime}$ frame observed from the $t$ frame. The velocity $v$ is the translational velocity of the $t^{\prime}$ frame observed from the rest frame located at the radius $r$ from the source of gravity. Elements $s$ and $m$ are the spin quantum number and mass of the particle of the $t^{\prime}$ frame observed from the $t^{\prime}$ frame. Elements $s^{\prime}$ and $m^{\prime}$ are the spin quantum number and mass of the particle of the $t^{\prime}$ frame observed from the $t$ frame.

Assumption 9.2: The spin quantum numbers $s$ and $s^{\prime}$ are the same.

$$
s=s^{\prime}
$$


From assumption 9.2, the quantized coordinate transformations of equation (8.26) are expressed as

$$
\frac{r^{\prime}}{r}=\frac{\Delta r^{\prime}}{\Delta r}=\frac{t^{\prime}}{t}=\frac{\Delta t^{\prime}}{\Delta t}=\frac{c^{\prime}}{\mathrm{c}}=\frac{m}{m^{\prime}}
$$

The notation of mass has been changed to $m$ and $m^{\prime}$.

Assumption 9.3: From equation (9.4), when observed from the $t$ frame, in both the $t$ frame and the $t^{\prime}$ frame, the mass of particles without active gravitational mass is expressed as

$$
m^{\prime}=m \times \frac{\mathrm{c}}{c^{\prime}} .
$$

We calculate kinetic energy based on these assumptions and premises.

We show the total kinetic energy of particles without active gravitational mass of the $t^{\prime}$ frame observed from the $t$ frame.

First, when observed from the $t$ frame, the particle without active gravitational mass of the $t^{\prime}$ frame is observed as having the mass $m^{\prime}=m \times \frac{\mathrm{c}}{c^{\prime}}$, the rotational velocity $c^{\prime}$ and the translational velocity $v^{\prime}$.

Second, from equation (9.1), the kinetic energy $E$ of particles without active gravitational mass is expressed as $E=m v^{2}$.

Third, from Newtonian mechanics, the total kinetic energy $E$ is expressed as

$$
E=E_{r}+E_{t}
$$

where $E_{r}$ is the rotational kinetic energy, and $E_{t}$ is the translational kinetic energy.

Therefore, when observed from the $t$ frame, the total kinetic energy of particles without active gravitational mass of the $t^{\prime}$ frame is expressed as follows.

$$
\begin{gathered}
E_{r}^{\prime}=m^{\prime} c^{2}, \\
E_{t}^{\prime}=m^{\prime} v^{\prime 2}, \\
E^{\prime}=E_{r}^{\prime}+E_{t}^{\prime}=m^{\prime} c^{2}+m^{\prime} v^{\prime 2},
\end{gathered}
$$

where $E_{r}^{\prime}, E_{t}^{\prime}$ and $E^{\prime}$ are the rotational kinetic energy, translational kinetic energy and total kinetic energy of particles without active gravitational mass of the $t^{\prime}$ frame observed from the $t$ frame.

When the particle without active gravitational mass belongs to the $t$ frame from the $t^{\prime}$ frame, we show the total kinetic energy of that particle observed from the $t$ frame. 
First, at this time, that particle observed from the $t$ frame is observed as having the mass $m^{\prime}=m \times \frac{c}{c^{\prime}}$, the rotational velocity $c$ and the translational velocity $v^{\prime}=0$. From assumption 9.3 , its mass in the $t$ frame is also $m^{\prime}=m \times \frac{c}{c^{\prime}}$. When that particle belongs to the same $t$ frame as the observer, from the relativity principle, its rotational speed is the speed of light c. Since the $t$ frame is the rest frame, its translational speed is zero.

Second, at this time, the kinetic energy of that particle observed from the $t$ frame is expressed as follows.

$$
\begin{gathered}
E_{r}=m^{\prime} \mathrm{c}^{2}, \\
E_{t}=0, \\
E=E_{r}+E_{t}=m^{\prime} \mathrm{c}^{2},
\end{gathered}
$$

where $E_{r}, E_{t}$ and $E$ are the rotational kinetic energy, translational kinetic energy and total kinetic energy of the particle without active gravitational mass at this time.

Third, at this time, from equation (9.2), the potential energy $U$ of gravity of that particle observed from the $t$ frame is expressed as

$$
U=-\frac{2 \mathrm{G} M m^{\prime}}{r}
$$

Therefore, when the particle without active gravitational mass belongs to the $t$ frame from the $t^{\prime}$ frame, the total energy of that particle observed from the $t$ frame is expressed as

$$
E+U=m^{\prime} \mathrm{c}^{2}-\frac{2 \mathrm{G} M m^{\prime}}{r} .
$$

We show the law of energy conservation between different reference frames. In the $t$ frame and the $t^{\prime}$ frame, the force acting on the particle without active gravitational mass is only the conservative force of gravity. For this reason, the total kinetic energy $E_{r}^{\prime}+E_{t}^{\prime}$ of the $t^{\prime}$ frame is the same as the total energy $E+U$ of the $t$ frame. Therefore, the following energy conservation law holds.

$$
\begin{gathered}
E^{\prime}=E_{r}^{\prime}+E_{t}^{\prime}=E+U . \\
E^{\prime}=m^{\prime} c^{\prime 2}+m^{\prime} v^{\prime 2}=m^{\prime} c^{2}-\frac{2 \mathrm{G} M m^{\prime}}{r} .
\end{gathered}
$$

The energy $E^{\prime}$ of equation (9.16) is the kinetic energy of not only particles without active gravitational mass but also particles with active gravitational mass. This is because the kinetic energy of all elementary particles is the sum of the kinetic energies of spacetime 
particles within the elementary particle. Therefore, we can calculate the kinetic energy of particle with active gravitational mass from equation (9.16).

However, particles without active gravitational mass, except for spacetime particles composing spacetime, have only rotational kinetic energy. This is because they propagate through a translating medium and they themselves do not translate. We explain this in section 10.

We discuss the physical meaning of the law of energy conservation for elementary particles.

When spacetime particles are confined within an elementary particle, their kinetic energy is expressed as $E_{r}^{\prime}+E_{t}^{\prime}=m^{\prime} c^{\prime 2}+m^{\prime} v^{\prime 2}$. This is because spacetime particles inside the elementary particle have translational kinetic energy.

When spacetime particles are emitted to the outside of an elementary particle as light, their kinetic energy is expressed as $E+U=m^{\prime} \mathrm{c}^{2}-\frac{2 \mathrm{G} M m^{\prime}}{r}$. This is because spacetime particles (= photons) outside the elementary particle do not have translational kinetic energy.

Therefore, the law of energy conservation for elementary particles means that the energy of spacetime particles inside the elementary particle and the energy of spacetime particles outside the elementary particle are the same. For this reason, the energy $E_{r}^{\prime}+E_{t}^{\prime}$ and $E+U$ have the same energy, but their physical meanings are different.

In this way, the law of energy conservation between different reference frames is derived.

\subsection{Spacetime Change and Kinetic Energy Caused by Translational Velocity}

We derive the spacetime change and kinetic energy caused by translational velocity.

Since only the translational velocity is considered, the potential energy $U$ of gravity is expressed as

$$
U=0 .
$$

Since both the $t$ frame and the $t^{\prime}$ frame are located at the same radius from the gravity source, the translational velocity $v^{\prime}$ is the same as the translational velocity $v$.

$$
v^{\prime}=v .
$$

Therefore, when only the translational velocity is considered, the law of energy conservation is expressed as

$$
E^{\prime}=m^{\prime} c^{\prime 2}+m^{\prime} v^{2}=m^{\prime} c^{2} .
$$

From equation (9.19), the velocity $c^{\prime}$ of radius or the rotational velocity $c^{\prime}$ is expressed as follows.

$$
c^{\prime 2}+v^{2}=\mathrm{c}^{2} .
$$




$$
c^{\prime}=c \sqrt{1-\left(\frac{v}{c}\right)^{2}} .
$$

From equations (9.4) and (9.21), the quantized coordinate transformations are expressed as

$$
\frac{r^{\prime}}{r}=\frac{\Delta r^{\prime}}{\Delta r}=\frac{t^{\prime}}{t}=\frac{\Delta t^{\prime}}{\Delta t}=\frac{c^{\prime}}{\mathrm{c}}=\frac{m}{m^{\prime}}=\sqrt{1-\left(\frac{v}{\mathrm{c}}\right)^{2}} .
$$

From equation (9.22), the mass $m^{\prime}$ is expressed as

$$
m^{\prime}=\frac{m}{\sqrt{1-\left(\frac{v}{c}\right)^{2}}}
$$

From equations (9.21) and (9.23), each kinetic energy is expressed as follows.

$$
\begin{gathered}
E_{r}^{\prime}=m^{\prime} c^{\prime 2}=m c^{2} \sqrt{1-\left(\frac{v}{c}\right)^{2}} . \\
E_{t}^{\prime}=m^{\prime} v^{2}=\frac{m v^{2}}{\sqrt{1-\left(\frac{v}{c}\right)^{2}}} . \\
E_{r}^{\prime}+E_{t}^{\prime}=\frac{m c^{2}}{\sqrt{1-\left(\frac{v}{c}\right)^{2}}} . \\
E=m^{\prime} c^{2}=\frac{m c^{2}}{\sqrt{1-\left(\frac{v}{c}\right)^{2}}}
\end{gathered}
$$

Therefore, when only the translational velocity is considered, the kinetic energy $E^{\prime}$ is expressed as

$$
E^{\prime}=\frac{m c^{2}}{\sqrt{1-\left(\frac{v}{c}\right)^{2}}} .
$$

Equation (9.28) is consistent with the kinetic energy of special relativity.

We discuss the kinetic energy at $v=0$. Each kinetic energy at $v=0$ is expressed as follows.

$$
\begin{gathered}
E_{r}^{\prime}=m^{\prime} c^{\prime 2}=m c^{2} \\
E_{t}^{\prime}=m^{\prime} v^{2}=0
\end{gathered}
$$




$$
\begin{gathered}
E^{\prime}=E_{r}^{\prime}+E_{t}^{\prime}=m \mathrm{c}^{2} . \\
E=m^{\prime} \mathrm{c}^{2}=m \mathrm{c}^{2} .
\end{gathered}
$$

Therefore, the kinetic energy $E^{\prime}=m c^{2}$ at $v=0$ represents the rotational kinetic energy at the speed of light. Although the energy $E^{\prime}=m \mathrm{c}^{2}$ is called the rest energy, the rest energy is the rotational kinetic energy at the speed of light. For this reason, even though the object is stationary, the unnaturalness of having kinetic energy is eliminated. That is, since an object is moving, it has kinetic energy.

We discuss the kinetic energy at $v \fallingdotseq \mathrm{c}$. Each kinetic energy at $v \fallingdotseq \mathrm{c}$ is expressed as follows.

$$
\begin{gathered}
E_{r}^{\prime}=m^{\prime} c^{\prime 2} \fallingdotseq 0 \\
E_{t}^{\prime}=m^{\prime} v^{2} \fallingdotseq \infty \\
E^{\prime}=E_{r}^{\prime}+E_{t}^{\prime} \fallingdotseq \infty \\
E=m^{\prime} \mathrm{c}^{2} \fallingdotseq \infty
\end{gathered}
$$

Therefore, when $v \fallingdotseq \mathrm{c}$, in the $t^{\prime}$ frame, the rotational kinetic energy $E_{r}^{\prime}$ is zero, and kinetic energy is only the translational kinetic energy $E_{t}^{\prime}$. That is, the rotational velocity or the velocity of radius is almost zero, and the translational velocity is almost the speed of light.

In this way, the spacetime change and kinetic energy caused by translational velocity are derived to be consistent with the relativity theory.

\subsection{Spacetime Change and Kinetic Energy Caused by Gravity}

We derive the spacetime change and kinetic energy caused by gravity.

Since only gravity is considered, the translational velocity $v^{\prime}$ and $v$ are zero.

$$
v^{\prime}=v=0 .
$$

Therefore, when only gravity is considered, the law of energy conservation is expressed as

$$
E^{\prime}=m^{\prime} c^{\prime 2}=m^{\prime} \mathrm{c}^{2}-\frac{2 \mathrm{G} M m^{\prime}}{r} .
$$


From equation (9.38), the velocity $c^{\prime}$ of radius or the rotational velocity $c^{\prime}$ is expressed as follows.

$$
\begin{aligned}
c^{2} & =c^{2}-\frac{2 \mathrm{G} M}{r} . \\
c^{\prime} & =c \sqrt{1-\frac{r_{s}}{r}},
\end{aligned}
$$

where $r_{s}$ is the Schwarzschild radius and is expressed as

$$
r_{s}=\frac{2 \mathrm{G} M}{\mathrm{c}^{2}}
$$

From equations (9.4) and (9.40), the quantized coordinate transformations are expressed as

$$
\frac{r^{\prime}}{r}=\frac{\Delta r^{\prime}}{\Delta r}=\frac{t^{\prime}}{t}=\frac{\Delta t^{\prime}}{\Delta t}=\frac{c^{\prime}}{\mathrm{c}}=\frac{m}{m^{\prime}}=\sqrt{1-\frac{r_{s}}{r}} .
$$

From equation (9.42), the mass $m^{\prime}$ is expressed as

$$
m^{\prime}=\frac{m}{\sqrt{1-\frac{r_{s}}{r}}}
$$

From equations (9.37) and (9.40) and (9.43), each energy is expressed as follows.

$$
\begin{gathered}
E_{r}^{\prime}=m^{\prime} c^{\prime 2}=m c^{2} \sqrt{1-\frac{r_{s}}{r}} . \\
E_{t}^{\prime}=m^{\prime} v^{2}=0 \\
E=m^{\prime} \mathrm{c}^{2}=\frac{m \mathrm{c}^{2}}{\sqrt{1-\frac{r_{s}}{r}}} . \\
U=-\frac{2 \mathrm{G} M m^{\prime}}{r}=-\frac{m \mathrm{c}^{2}}{\sqrt{1-\frac{r_{s}}{r}}} \times \frac{r_{s}}{r} . \\
E_{r}^{\prime}+E_{t}^{\prime}=m \mathrm{c}^{2} \sqrt{1-\frac{r_{s}}{r}} . \\
E+U=m \mathrm{c}^{2} \sqrt{1-\frac{r_{s}}{r}} .
\end{gathered}
$$

Therefore, when only gravity is considered, the kinetic energy $E^{\prime}$ is expressed as 


$$
E^{\prime}=m c^{2} \sqrt{1-\frac{r_{s}}{r}}
$$

We show gravitational redshift. The kinetic energy considering only gravity does not include translational kinetic energy, so it is also the energy of light. From $E=m c^{2}=\mathrm{h} f$, equation (9.50) is expressed as

$$
E^{\prime}=m c^{2} \sqrt{1-\frac{r_{s}}{r}}=\mathrm{h} f \sqrt{1-\frac{r_{s}}{r}} .
$$

We set the frequency $f^{\prime}$ as

$$
f^{\prime}=f \sqrt{1-\frac{r_{s}}{r}}
$$

where $f^{\prime}$ is the frequency of light when the light in radius $r$ from the source of gravity reaches infinity distance, $f$ is the frequency of light in radius $r$ from the source of gravity. Then, the wavelength $\lambda^{\prime}$ of $f^{\prime}$ and the wavelength $\lambda$ of $f$ are expressed as

$$
\begin{aligned}
\lambda^{\prime} & =\frac{\mathrm{c}}{f^{\prime}}, \\
\lambda & =\frac{\mathrm{c}}{f} .
\end{aligned}
$$

From these, the wavelength $\lambda^{\prime}$ is expressed as

$$
\lambda^{\prime}=\frac{\lambda}{\sqrt{1-\frac{r_{s}}{r}}} .
$$

The wavelength $\lambda^{\prime}$ is the wavelength of light by gravitational redshift. Equation (9.55) is consistent with the relativity theory.

In this way, the spacetime change and kinetic energy caused by gravity are derived to be consistent with the relativity theory.

\subsection{Spacetime Change and Kinetic Energy Caused by Translational Velocity and Gravity}

We derive the spacetime change and kinetic energy caused by translational velocity and gravity.

When the translational velocity and gravity are considered, the law of energy conservation is expressed as $E^{\prime}=m^{\prime} c^{\prime 2}+m^{\prime} v^{\prime 2}=m^{\prime} c^{2}-\frac{2 \mathrm{G} M m^{\prime}}{r}$ of equation (9.16). 
When only gravity is considered, the velocity $c^{\prime}$ of radius or the rotational velocity $c^{\prime}$ is expressed as $c^{\prime}=c \sqrt{1-\frac{r_{s}}{r}}$ of equation (9.40). Similarly, the translational velocity $v^{\prime}$ is expressed as

$$
v^{\prime}=v \sqrt{1-\frac{r_{s}}{r}} .
$$

Therefore, when translational velocity and gravity is considered, from equations (9.16) and (9.56), the velocity $c^{\prime}$ of radius or the rotational velocity $c^{\prime}$ is expressed as

$$
c^{\prime}=\mathrm{c} \sqrt{1-\left(\frac{v}{\mathrm{c}}\right)^{2}} \sqrt{1-\frac{r_{s}}{r}} .
$$

From equations (9.4) and (9.57), the quantized coordinate transformations are expressed as

$$
\frac{r^{\prime}}{r}=\frac{\Delta r^{\prime}}{\Delta r}=\frac{t^{\prime}}{t}=\frac{\Delta t^{\prime}}{\Delta t}=\frac{c^{\prime}}{\mathrm{c}}=\frac{m}{m^{\prime}}=\sqrt{1-\left(\frac{v}{\mathrm{c}}\right)^{2}} \sqrt{1-\frac{r_{s}}{r}} .
$$

This spacetime change is consistent with the relativity theory.

From equation (9.58), the mass $m^{\prime}$ is expressed as

$$
m^{\prime}=\frac{m}{\sqrt{1-\left(\frac{v}{c}\right)^{2}} \sqrt{1-\frac{r_{s}}{r}}} .
$$

From equations (9.56) and (9.57) and (9.59), each energy is expressed as follows.

$$
\begin{gathered}
E_{r}^{\prime}=m^{\prime} c^{\prime 2}=m \mathrm{c}^{2} \sqrt{1-\left(\frac{v}{\mathrm{c}}\right)^{2}} \sqrt{1-\frac{r_{s}}{r}} . \\
E_{t}^{\prime}=m^{\prime} v^{\prime 2}=m v^{2} \frac{\sqrt{1-\frac{r_{s}}{r}}}{\sqrt{1-\left(\frac{v}{\mathrm{c}}\right)^{2}}} . \\
E=m^{\prime} \mathrm{c}^{2}=\frac{m \mathrm{c}^{2}}{\sqrt{1-\left(\frac{v}{\mathrm{c}}\right)^{2}} \sqrt{1-\frac{r_{s}}{r}}} . \\
U=-\frac{2 \mathrm{GM} m^{\prime}}{r}=-\frac{m \mathrm{c}^{2}}{\sqrt{1-\left(\frac{v}{\mathrm{c}}\right)^{2}} \sqrt{1-\frac{r_{s}}{r}}} \times \frac{r_{s}}{r} . \\
E_{r}^{\prime}+E_{t}^{\prime}=m \mathrm{c}^{2} \frac{\sqrt{1-\frac{r_{s}}{r}}}{\sqrt{1-\left(\frac{v}{\mathrm{c}}\right)^{2}}} . \\
E+U=m \mathrm{c}^{2} \frac{\sqrt{1-\frac{r_{s}}{r}}}{\sqrt{1-\left(\frac{v}{\mathrm{c}}\right)^{2}}} .
\end{gathered}
$$


Therefore, when translational velocity and gravity is considered, the kinetic energy $E^{\prime}$ is expressed as

$$
E^{\prime}=m c^{2} \frac{\sqrt{1-\frac{r_{s}}{r}}}{\sqrt{1-\left(\frac{v}{c}\right)^{2}}} .
$$

In this way, the spacetime change caused by translational velocity and gravity is derived to be consistent with the relativity theory, and kinetic energy is derived.

In summary, from the law of energy conservation between different reference frames and the quantized coordinate transformations, the spacetime change and kinetic energy caused by translational velocity and gravity are derived. The idea is different from the relativity theory, but the result is consistent with the relativity theory. In this paper, the rest energy $E=m c^{2}$ can be explained as rotational kinetic energy at the speed of light, and the spacetime change can be explained by the law of energy conservation of spacetime particles composing spacetime.

\section{Doppler Effect for Light and Energy of Light}

In this section, we discuss the Doppler effect for light and the energy of light. As an observation of light affected by spacetime change caused by translational velocity, there is the Doppler effect for light. For this reason, from the Doppler effect for light, we can understand the properties of light. Moreover, from the Doppler effect for sound, we can understand the Doppler effect for light. Therefore, we discuss the energy of light from the Doppler effect for light and sound.

\subsection{Doppler Effect for Sound}

We explain the Doppler effect for sound to understand the Doppler effect for light. They are the same Doppler effect, so they need to be explained in the same way.

We show the premises of the Doppler effect for sound.

First, the medium of air that propagates sound waves is stationary.

Second, the following equation holds for sound waves.

$$
\begin{gathered}
v_{s}=f \lambda, \\
f=\frac{v_{s}}{\lambda}=\frac{1}{T}, \\
T=\frac{\lambda}{v_{s}},
\end{gathered}
$$




$$
\begin{gathered}
v_{s}^{\prime}=f^{\prime} \lambda^{\prime}, \\
f^{\prime}=\frac{v_{s}^{\prime}}{\lambda^{\prime}}=\frac{1}{T^{\prime}}, \\
T^{\prime}=\frac{\lambda^{\prime}}{v_{s}^{\prime}},
\end{gathered}
$$

where $v_{s}, \lambda, f$ and $T$ are the velocity, wavelength, frequency and period of sound waves observed from an observer when the observer and a sound source are stationary, and $v_{s}^{\prime}, \lambda^{\prime}$, $f^{\prime}$ and $T^{\prime}$ are the velocity, wavelength, frequency and period of sound waves observed from the observer when the observer and the sound source are at any velocity.

We explain the Doppler effect for sound when the sound source moves away from the observer at velocity $v$ against the stationary medium.

First, we show the velocity $v_{s}^{\prime}$ of sound waves observed from the observer. Even if the sound source moves at velocity $v$, the medium of air that propagates sound waves remains stationary, so waves propagate through the medium at a constant velocity. For this reason, the velocity of sound waves from the sound source is constant regardless of velocity $v$. Therefore, the velocity $v_{s}^{\prime}$ of sound waves observed from the observer is expressed as

$$
v_{s}^{\prime}=v_{s} .
$$

Second, we show the wavelength $\lambda^{\prime}$ of sound waves observed from the observer. A wave propagates through the medium in a spherical shape centered on the generation point, and after it is generated, it is not affected by the wave source. Therefore, when the wave source moves at velocity $v$ against the medium, the wavelength changes depending on the direction. Since the sound source moves by the distance of $v T$ during one period $T$ of sound waves, its wavelength is extended by $v T$. Therefore, the wavelength $\lambda^{\prime}$ of sound waves observed from the observer is expressed as

$$
\lambda^{\prime}=\lambda+v T=\lambda\left(1+\frac{v}{v_{s}}\right) .
$$

When the sound source moves away from the observer at velocity $v$ against the stationary medium, from equations (10.7) and (10.8), the frequency $f^{\prime}$ of sound waves observed from the observer is expressed as

$$
f^{\prime}=\frac{v_{s}^{\prime}}{\lambda^{\prime}}=\frac{f}{1+\frac{v}{v s}} .
$$

We explain the Doppler effect for sound when the observer moves away from the sound source at velocity $v_{o}$ against the stationary medium. 
First, we show the wavelength $\lambda^{\prime}$ of sound waves observed from the observer. Even if the observer moves at velocity $v_{o}$, the sound source remains stationary and the wave generation point does not move against the medium. For this reason, the wavelength of sound waves does not change depending on velocity $v_{o}$. Therefore, the wavelength $\lambda^{\prime}$ of sound waves observed from the observer is expressed

$$
\lambda^{\prime}=\lambda
$$

Second, we show the velocity $v_{s}^{\prime}$ of sound waves observed from the observer. Sound waves propagate through the medium at a constant velocity $v_{s}$. The observer moves away from the sound source at velocity $v_{o}$ against the stationary medium. Therefore, the velocity $v_{s}^{\prime}$ of sound waves observed from the observer is expressed

$$
v_{s}^{\prime}=v_{s}-v_{o} .
$$

When the observer moves away from the sound source at velocity $v_{o}$ against the stationary medium, from equations (10.10) and (10.11), the frequency $f^{\prime}$ of sound waves observed from the observer is expressed as

$$
f^{\prime}=\frac{v_{s}^{\prime}}{\lambda^{\prime}}=f\left(1-\frac{v_{o}}{v_{s}}\right)
$$

We explain the Doppler effect for sound when the sound source and the observer move away from each other.

The sound source moves away from the observer at velocity $v$ against the stationary medium. The observer moves away from the sound source at velocity $v_{o}$ against the stationary medium. At this time, the wavelength $\lambda^{\prime}$ and velocity $v_{s}^{\prime}$ of sound waves observed from the observer are expressed by equations (10.8) and (10.11). This is because, from the above explanation, the Doppler effect is caused by velocity against the stationary medium. Therefore, when the sound source and the observer move away from each other, from equations (10.8) and (10.11), the frequency $f^{\prime}$ of sound waves observed from the observer is expressed as

$$
f^{\prime}=\frac{v_{s}^{\prime}}{\lambda^{\prime}}=f \times \frac{1-\frac{v_{o}}{v_{s}}}{1+\frac{v}{v_{s}}} .
$$

In this way, the Doppler effect for sound is explained. Importantly, the Doppler effect is caused by velocity against the stationary medium.

\subsection{Doppler Effect for Light}

We explain the Doppler effect for light. 
First of all, we show that light propagates through a medium from its energy.

First, from equations (9.24) and (9.25) and $m \mathrm{c}^{2}=\mathrm{h} f$, the rotational kinetic energy $E_{r}^{\prime}$ and translational kinetic energy $E_{t}^{\prime}$ caused by translational velocity are expressed as

$$
\begin{aligned}
& E_{r}^{\prime}=m^{\prime} c^{\prime 2}=m \mathrm{c}^{2} \sqrt{1-\left(\frac{v}{\mathrm{c}}\right)^{2}}=\mathrm{h} f \sqrt{1-\left(\frac{v}{\mathrm{c}}\right)^{2}} . \\
& E_{t}^{\prime}=m^{\prime} v^{2}=\frac{m v^{2}}{\sqrt{1-\left(\frac{v}{\mathrm{c}}\right)^{2}}}=\frac{\mathrm{h} f}{\sqrt{1-\left(\frac{v}{\mathrm{c}}\right)^{2}}} \times\left(\frac{v}{\mathrm{c}}\right)^{2} .
\end{aligned}
$$

Second, when the direction of motion of a light source is perpendicular to the direction from an observer to the light source, from the observation or the Doppler effect for light, the energy $h f^{\prime}$ of the light from the light source with velocity $v$ is expressed as

$$
\mathrm{h} f^{\prime}=\mathrm{h} f \sqrt{1-\left(\frac{v}{c}\right)^{2}},
$$

where $f^{\prime}$ is the frequency of the light from the light source with velocity $v, f$ is is the frequency of the light from the light source with velocity $v=0$.

The light energy of equation (10.16) is the same as that of equation (10.14). At this time, if the light source is a sound source, the velocity of the distance between the wave source and the observer is zero, so the Doppler effect does not occur. Therefore, the light energy of equation (10.16) is the kinetic energy caused by the translational velocity without the Doppler effect. Moreover, from equations (10.14) and (10.16), we conclude that light has only the rotational kinetic energy $E_{r}^{\prime}$. For this reason, the translational kinetic energy $E_{t}^{\prime}$ of light is zero.

$$
E_{t}^{\prime}=0
$$

Since the light is from the light source with velocity $v$, the light belongs to the $t^{\prime}$ frame moving at the translational velocity $v$. When an elementary particle moves at the translational velocity $v$, the light confined within the elementary particle has translational kinetic energy. Nevertheless, the light from the light source with velocity $v$ has no translational kinetic energy. This is because the light propagates through the medium moving at the translational velocity $v$, and the light itself does not translate. Note that the speed of light is the speed of radius or the rotational speed, not the translational speed. Therefore, we conclude that light propagates through the medium from its energy. If light itself translates, light must have translational kinetic energy.

The medium of light is spacetime particles composing spacetime. First, although light propagates through a vacuum, the vacuum is spacetime particles composing spacetime. Second, spacetime particles composing spacetime translate at velocity $v$ with matter. Third, 
from the properties of particles without active gravitational mass, light belongs to the spacetime of matter with active gravitational mass. Therefore, we conclude that the medium of light is spacetime particles composing spacetime.

From the above, we show the assumption and premise of the Doppler effect for light.

Assumption 10.1: The light emitted from the light source belongs to all the media existing in space at the same time, and each observer observes it as belonging to his own stationary medium.

The light emitted from a light source is observed by all observers as the speed of light, regardless of the speed of the light source. Light propagates through the medium of spacetime particles. Since spacetime particles belonging to an observer translate with the observer at the same velocity as the observer, the observer observes them as if they didn't translate and were always stationary. When light propagates through a stationary medium, the observer observes its speed of light as a constant speed. Therefore we conclude assumption 10.1.

As a premise, we set the angle between the direction of motion of the light source and the direction from the light source to an observer as $\theta$, and the following equation holds for light.

$$
\begin{gathered}
c=f \lambda, \\
f=\frac{c}{\lambda}=\frac{1}{T}, \\
T=\frac{\lambda}{c}, \\
c^{\prime}=f^{\prime} \lambda^{\prime}, \\
f^{\prime}=\frac{c^{\prime}}{\lambda^{\prime}}=\frac{1}{T^{\prime}}, \\
T^{\prime}=\frac{\lambda^{\prime}}{c^{\prime}}, \\
c^{\prime \prime}=f^{\prime \prime} \lambda^{\prime \prime}, \\
f^{\prime \prime}=\frac{c^{\prime \prime}}{\lambda^{\prime \prime}}=\frac{1}{T^{\prime \prime}}, \\
T^{\prime \prime}=\frac{\lambda^{\prime \prime}}{c^{\prime \prime}},
\end{gathered}
$$


where c, $f, \lambda$ and $T$ are the speed, frequency, wavelength and period of light observed from the observer when the observer and the light source are stationary, $c^{\prime}, f^{\prime}, \lambda^{\prime}$ and $T^{\prime}$ are the speed, frequency, wavelength and period of light at $\theta=\frac{\pi}{2}$ observed from the observer when the observer and the light source are at any velocity, $c^{\prime \prime}, f^{\prime \prime}, \lambda^{\prime \prime}$ and $T^{\prime \prime}$ are the speed, frequency, wavelength and period of light at $\theta=\pi$ observed from the observer when the observer and the light source are at any velocity.

We explain the Doppler effect for light when the light source moves away from the observer at velocity $v$ and $\theta=\pi$.

From the Doppler effect for sound, the Doppler effect is caused by velocity against the stationary medium. From assumption 10.1, even if the observer moves, it is the light source that is moving against the stationary medium belonging to the observer. Therefore, we only need to consider when the light source moves.

First, we show the light speeds $c^{\prime}$ and $c^{\prime \prime}$ observed from the observer. Even if the light source moves at velocity $v$, when the observer observes the medium belonging to the observer, that medium remains stationary. Light propagates through the medium at a constant light speed c. For this reason, the speed of light from the light source is constant regardless of the light source velocity $v$. Therefore, the light speeds $c^{\prime}$ and $c^{\prime \prime}$ observed from the observer are expressed as

$$
c^{\prime}=c^{\prime \prime}=\mathrm{c} .
$$

Second, we show the frequency $f^{\prime}$, wavelength $\lambda^{\prime}$ and period $T^{\prime}$ of light at $\theta=\frac{\pi}{2}$ observed from the observer. From equation (10.16), the frequency $f^{\prime}$ of light at $\theta=\frac{\pi}{2}$ is expressed as

$$
f^{\prime}=f \sqrt{1-\left(\frac{v}{c}\right)^{2}} .
$$

From equations (10.27) and (10.28), the wavelength $\lambda^{\prime}$ and period $T^{\prime}$ of light at $\theta=\frac{\pi}{2}$ are expressed as

$$
\begin{aligned}
\lambda^{\prime} & =\frac{c^{\prime}}{f^{\prime}}=\frac{\lambda}{\sqrt{1-\left(\frac{v}{c}\right)^{2}}}, \\
T^{\prime} & =\frac{\lambda^{\prime}}{c^{\prime}}=\frac{T}{\sqrt{1-\left(\frac{v}{c}\right)^{2}}} .
\end{aligned}
$$

Third, we show the wavelength $\lambda^{\prime \prime}$ and frequency $f^{\prime \prime}$ of light at $\theta=\pi$ observed from the observer. The light emitted from the light source has the wavelength $\lambda^{\prime}$, the period $T^{\prime}$ and the light speed c. A wave propagates through the medium in a spherical shape centered on the generation point, and after it is generated, it is not affected by the wave source. For this reason, when the wave generation point moves against the medium, the wavelength changes 
depending on the direction. Since the light source moves by the distance of $v T^{\prime}$ during one period $T^{\prime}$ of the light, its wavelength is extended by $v T^{\prime}$. Therefore, the wavelength $\lambda^{\prime \prime}$ of light at $\theta=\pi$ observed from the observer is expressed as

$$
\lambda^{\prime \prime}=\lambda^{\prime}+v T^{\prime}=\lambda \times \frac{1+\frac{v}{\mathrm{c}}}{\sqrt{1-\left(\frac{v}{\mathrm{c}}\right)^{2}}} .
$$

From equations (10.27) and (10.31), the frequency $f^{\prime \prime}$ of light at $\theta=\pi$ observed from the observer is expressed as

$$
f^{\prime \prime}=\frac{c^{\prime \prime}}{\lambda^{\prime \prime}}=f \times \frac{\sqrt{1-\left(\frac{v}{c}\right)^{2}}}{1+\frac{v}{c}} .
$$

In this way, the Doppler effect for light is explained. In the case of the Doppler effect for light, the observer cannot move against the medium.

\subsection{Energy of Light}

We show the energy of light.

First of all, we show the premise of light energy.

First, from assumption 2.10, in the energy of light, the mass $m$ of light is expressed as $m=\frac{\mathrm{h} f}{\mathrm{c}^{2}}$.

Second, light has only rotational kinetic energy, and the translational kinetic energy $E_{t}^{\prime}$ of light is zero $\left(E_{t}^{\prime}=0\right)$. Even if the light in the $t^{\prime}$ frame appears to have translational velocity $v$, that translational velocity $v$ is the velocity of the medium, and the light itself does not move at the translational velocity $v$.

Third, we consider only the case of $\theta=\frac{\pi}{2}$. An angle $\theta$ is the angle between the direction of motion of a light source and the direction from the light source to an observer.

From these three premises, we show the energy of light. In the spacetime change caused by translational velocity, from equation (9.24), the energy $E^{\prime}$ of light is expressed as

$$
E^{\prime}=E_{r}^{\prime}=m^{\prime} c^{2}=m c^{2} \sqrt{1-\left(\frac{v}{c}\right)^{2}}=\mathrm{h} f \sqrt{1-\left(\frac{v}{\mathrm{c}}\right)^{2}} .
$$

In the spacetime change caused by gravity, from equation (9.44), the energy $E^{\prime}$ of light is expressed as

$$
E^{\prime}=E_{r}^{\prime}=m^{\prime} c^{\prime 2}=m c^{2} \sqrt{1-\frac{r_{s}}{r}}=\mathrm{h} f \sqrt{1-\frac{r_{s}}{r}} .
$$

In the spacetime change caused by translational velocity and gravity, from equation (9.60), the energy $E^{\prime}$ of light is expressed as 


$$
E^{\prime}=E_{r}^{\prime}=m^{\prime} c^{\prime 2}=m c^{2} \sqrt{1-\left(\frac{v}{c}\right)^{2}} \sqrt{1-\frac{r_{s}}{r}}=\mathrm{h} f \sqrt{1-\left(\frac{v}{\mathrm{c}}\right)^{2}} \sqrt{1-\frac{r_{s}}{r}}
$$

In this way, the energy of light is shown. This energy of light is also the energy of particles without active gravitational mass, except for spacetime particles composing spacetime.

\subsection{Time and Space Perceived by Light}

From the energy of light, we explain the time and space perceived by light. In the spacetime change caused by translational velocity, the energy $E^{\prime}$ of light at $\theta=\frac{\pi}{2}$ is expressed by equation (10.33). Since the energy $E$ of light at $v=0$ is expressed as $E=m c^{2}=\mathrm{h} f$, this light belongs to the rest frame of $v=0$ from the beginning. Note that even if $v=0$, the radius of light changes at the speed of light, and the position of light appears to change at the speed of light by wave function reduction. The time $t^{\prime}$ and space $r^{\prime}$ perceived by light at $v=0$ are expressed as follows from equation (9.22).

$$
\begin{aligned}
& t^{\prime}=t \sqrt{1-\left(\frac{v}{c}\right)^{2}}=t . \\
& r^{\prime}=r \sqrt{1-\left(\frac{v}{c}\right)^{2}}=r .
\end{aligned}
$$

From equations (10.36) and (10.37), even if the radius of light is changing at the speed of light and the position of light appears to change at the speed of light by wave function reduction, light perceives time and space because its translational velocity $v$ is zero $(v=0)$.

In summary, light propagates through the medium of spacetime particles that translates with the observer, and the Doppler effect for light can be explained in the same way as the Doppler effect for sound. Light has only rotational kinetic energy, and light itself does not translate. The speed of light is not the translational speed but the speed of the radius, and the light belonging to the rest frame perceives time and space because its translational velocity $v$ is zero $(v=0)$.

\section{Black Holes and Gravity}

In this section, we explain black holes and gravity. Although we derive black holes, gravitons in this paper have mass, so they cannot escape from black holes. For this reason, the gravity of black holes is not exerted outside the Schwarzschild radius. We solve this black hole problem. 


\subsection{Forces Exerted on Particles Without Active Gravitational Mass}

We show forces exerted on particles without active gravitational mass. From the potential energy of equation (9.2), gravity exerted on particles without active gravitational mass is expressed as

$$
F=-\frac{\mathrm{d} U}{\mathrm{~d} r}=-\frac{2 \mathrm{G} M m}{r^{2}} .
$$

Similarly, from equation (7.12), three forces received by mass of particles without active gravitational mass are expressed as

$$
F=-\frac{2 \mathrm{G} M m}{r^{2}}-\frac{2 \mathrm{G} M m}{r \times \mathrm{r}_{\mathrm{e}}}+2 m r A_{P} .
$$

Therefore, particles without active gravitational mass receive twice as much force as particles with active gravitational mass.

\subsection{The Radius of Gravity That Cannot Escape Even at the Speed of Light}

We show the radius of gravity that cannot escape even at the speed of light. That radius is the radius at which the sum of the kinetic energy $E$ of equation (9.62) and the potential energy $U$ of the gravity of equation (9.63) becomes zero. Therefore, that radius $r$ is expressed as follows.

$$
\begin{gathered}
E+U=m c^{2} \frac{\sqrt{1-\frac{r_{s}}{r}}}{\sqrt{1-\left(\frac{v}{\mathrm{c}}\right)^{2}}}=0 . \\
r=r_{s}=\frac{2 \mathrm{G} M}{\mathrm{c}^{2}} .
\end{gathered}
$$

From equation (11.4), that radius $r$ is the Schwarzschild radius $r_{s}$. From equation (9.58), the quantized coordinate transformations at $r=r_{s}$ are expressed as

$$
\frac{r^{\prime}}{r}=\frac{\Delta r^{\prime}}{\Delta r}=\frac{t^{\prime}}{t}=\frac{\Delta t^{\prime}}{\Delta t}=\frac{c^{\prime}}{\mathrm{c}}=0 .
$$

Therefore, at $r=r_{s}$, the speed of light and time appear to stop because of $c^{\prime}=0$ and $t^{\prime}=0$. These are consistent with the theory of relativity. From these, when the radius $r$ of matter is $r<r_{s}$, the space of $r \leqq r_{s}$ becomes a black hole in which even light cannot escape. 


\subsection{The Gravity of a Black Hole and How to Calculate the Effect of Spacetime Change}

We show the gravity of a black hole and how to calculate the effect of spacetime change.

In this section, the $t$ frame is the rest frame located at infinity from the source of gravity, and the $t^{\prime}$ frame is the rest frame located at the radius $r$ from the source of gravity.

From the spacetime change of equation (9.42), the radius $r^{\prime}$ of the $t^{\prime}$ frame observed from the $t$ frame is expressed as

$$
r^{\prime}=r \sqrt{1-\frac{r_{s}}{r}}
$$

Therefore, the gravity $F^{\prime}$ of a black hole of the $t^{\prime}$ frame observed from the $t$ frame is expressed as

$$
F^{\prime}=-\frac{\mathrm{G} M m}{r^{\prime 2}}=-\frac{\mathrm{G} M m}{r^{2}\left(1-\frac{r_{s}}{r}\right)} .
$$

From equation (11.7), the gravity of a black hole at $r=r_{s}$ is infinite and singularity.

From these, we summarize how to calculate the effect of spacetime change. In the calculations of kinetic energy and momentum, we transform the mass and velocity of the $t^{\prime}$ frame into the physical quantity observed from the $t$ frame. In other calculations, we transform the distance and time of the $t^{\prime}$ frame or the physical quantity related to them of the $t^{\prime}$ frame into the physical quantity observed from the $t$ frame. If we do this way, we can calculate to match the observations.

\subsection{The Radius of Matter Within Black Holes and the Spacetime Radius of Black Holes}

Gravitons in this paper have mass, so they cannot escape from black holes. For this reason, the gravity of black holes is not exerted outside the Schwarzschild radius. To solve this black hole problem, we discuss the radius of matter within black holes and the spacetime radius of black holes.

We discuss the state of matter within black holes. In a black hole, its gravity causes the matter to contract to the limit. However, no matter how much the matter within black holes contracts, it is up to the minimum radius $\frac{\Delta r}{2}$. This is because gravity works only up to that radius. When the matter within black holes contracts to the minimum radius $\frac{\Delta r}{2}$, we can

consider the matter as one elementary particle. This is because the minimum radius $\frac{\Delta r}{2}$ is the radius that only one elementary particle can have. Therefore, we assume as follows.

Assumption 11.1: The matter within black holes turns into one elementary particle.

We call it the black hole elementary particle. 
In section 11.4, the individual elements have the following meanings: The $t$ frame is the rest frame located at infinity from the source of gravity. The $t^{\prime}$ frame is the rest frame located at the radius $r$ from the source of gravity. Elements $r, \Delta r$ and $r_{0}$ are the spacetime radius, quantized minimum radius and elementary particle radius of the black hole elementary particle of the $t^{\prime}$ frame observed from the $t^{\prime}$ frame. Elements $r^{\prime}, \Delta r^{\prime}$ and $r_{0}^{\prime}$ are the spacetime radius, quantized minimum radius and elementary particle radius of the black hole elementary particle of the $t^{\prime}$ frame observed from the $t$ frame.

We discuss the elementary particle radius of black hole elementary particles after the birth of the universe. For the time being, we assume that the elementary particle radius of black hole elementary particles is $\frac{\Delta r}{2}$. In this case, its minimum spacetime radius is less than the Schwarzschild radius $r_{s}$. Within the radius of less than $r_{s}$, gravitons with mass cannot escape. For this reason, the gravity of black holes is not exerted outside the Schwarzschild radius. However, phenomena caused by the gravity of black holes have been observed. Therefore, we conclude that the elementary particle radius of black hole elementary particles is not $\frac{\Delta r}{2}$, at least after the birth of the universe.

In conclusion, the elementary particle radius $r_{0}$ of black hole elementary particles after the birth of the universe is the Schwarzschild radius $r_{s}$.

$$
\begin{gathered}
r_{0}=r_{s}\left(\frac{\Delta r}{2}<r_{s}\right) . \\
r_{0}^{\prime}=r_{s}^{\prime}=r_{s} \sqrt{1-\frac{r_{s}}{r}}\left(\frac{\Delta r^{\prime}}{2}<r_{s}^{\prime}\right) .
\end{gathered}
$$

First, from the simplicity principle and the parsimony principle, it is preferable to explain from the theory so far, and the theory to explain only this is not preferable.

Second, from wave function reduction, the elementary particle radius changes flexibly depending on the situation. That is, the elementary particle radius is not always $\frac{\Delta r}{2}$.

Third, from equation (11.7), the gravity $F^{\prime}$ of black holes works as follows. If $r>r_{s}$, the attractive force of $F^{\prime}<0$ works. If $r<r_{s}$, the repulsive force of $F^{\prime}>0$ works. If $r=r_{s}+\Delta r$, the attractive force near infinity works. If $r=r_{s}-\Delta r$, the repulsive force near infinity works.

Fourth, black hole elementary particles after the birth of the universe are made by contracting from $r_{0}>r_{s}$ to $r_{0} \leqq r_{s}$. For this reason, that process is shown as follows. When its radius contracts from $r_{0}>r_{s}$ to $r_{0}=r_{s}-\Delta r$, the repulsive force near infinity works on the black hole elementary particle. Then when its radius expands from $r_{0}<r_{s}$ to $r_{0}=r_{s}+\Delta r$ by the repulsive force, the attractive force near infinity works on the black hole elementary particle. This is repeated and its radius oscillates for a while, but finally its radius stops at $r_{0}=r_{s}$.

Fifth, if the elementary particle radius of black hole elementary particles is $r_{s}$, its spacetime radius is larger than $r_{s}$, so no singularity is generated and gravitons can be emitted from 
black hole elementary particles to the outside the Schwarzschild radius $r_{s}$.

Therefore, we conclude that the elementary particle radius of black hole elementary particles after the birth of the universe is the Schwarzschild radius $r_{s}$.

We show the spacetime radius of black hole elementary particles after the birth of the universe. The general equation of the spacetime radius for any elementary particle radius is expressed as follows.

$$
\begin{aligned}
r & =\left(N-\frac{1}{2}\right) \Delta r+r_{0} . \\
r^{\prime} & =\left(N-\frac{1}{2}\right) \Delta r^{\prime}+r_{0}^{\prime} .
\end{aligned}
$$

Substituting equation (11.8) into equation (11.10), it is expressed as

$$
r=\left(N-\frac{1}{2}\right) \Delta r+r_{s}\left(\frac{\Delta r}{2}<r_{s}\right) .
$$

Substituting equation (11.9) into equation (11.11), it is expressed as

$$
r^{\prime}=\left(\left(N-\frac{1}{2}\right) \Delta r+r_{s}\right) \sqrt{1-\frac{r_{s}}{r}}\left(\frac{\Delta r^{\prime}}{2}<r_{s}^{\prime}\right) .
$$

Substituting $N=0$ into equation (11.12), from $\frac{\Delta r}{2}<r_{s}$, it is expressed as

$$
r(N=0)=r_{s}-\frac{\Delta r}{2}>0 .
$$

Although the spacetime radius does not become zero, it is less than the elementary particle radius $r_{s}$, so the spacetime radius does not exist within the elementary particle radius.

We discuss the presence or absence of singularity near the Schwarzschild radius after the birth of the universe. The minimum spacetime radius and its time are expressed as follows.

$$
\begin{gathered}
r(N=1)=\frac{\Delta r}{2}+r_{s} . \\
r^{\prime}(N=1)=\sqrt{\frac{\Delta r}{2}\left(\frac{\Delta r}{2}+r_{s}\right)} . \\
t(N=1)=\frac{r(N=1)}{\mathrm{c}}=\frac{\Delta t}{2}+\frac{r_{s}}{\mathrm{c}} . \\
t^{\prime}(N=1)=\frac{r^{\prime}(N=1)}{\mathrm{c}}=\sqrt{\frac{\Delta t}{2}\left(\frac{\Delta t}{2}+\frac{r_{s}}{\mathrm{c}}\right) .}
\end{gathered}
$$


The minimum spacetime radius of equation (11.15) is as close to the Schwarzschild radius as possible, but not the Schwarzschild radius itself. The spacetime radius of equation (11.16) is not zero. For this reason, gravitons at the speed of light can be emitted from black holes, and there is no black hole that nothing can escape from it at least after the birth of the universe.

From equations (11.7) and (11.16), the gravity $F^{\prime}$ of black holes at the smallest spacetime radius is expressed as

$$
F^{\prime}(N=1)=-\frac{\mathrm{G} M m}{\frac{\Delta r}{2}\left(\frac{\Delta r}{2}+r_{s}\right)} .
$$

From equation (11.19), the gravity $F^{\prime}$ of black holes does not become infinite. The quantized coordinate transformations of equation (9.42) at $N=1$ are expressed as

$$
\frac{r^{\prime}}{r}=\frac{\Delta r^{\prime}}{\Delta r}=\frac{t^{\prime}}{t}=\frac{\Delta t^{\prime}}{\Delta t}=\frac{c^{\prime}}{\mathrm{c}}=\frac{m}{m^{\prime}}=\sqrt{1-\frac{r_{s}}{\frac{\Delta r}{2}+r_{s}}} .
$$

From equation (11.20), the quantized coordinate transformations do not become zero, so no singularity occurs.

We discuss the radius and rotational speed of the actual matter within black holes after the birth of the universe. From $r_{0}=r_{s}$, the inside of $r_{s}$ is within the elementary particle radius of black hole elementary particles, so there is no graviton. For this reason, black hole elementary particles of $r_{0}=r_{s}$ rotate at the speed of light. However, in reality, black holes are rotating at less than the speed of light. In this case, the matter within black holes is not a single elementary particle but a group of matter. Owing to an inertial force, a group of matter rotates at less than the speed of light. Black hole elementary particles are in an ideal black hole state. Therefore, actual black holes are not necessarily black hole elementary particles, do not necessarily rotate at the speed of light, and their radius is not necessarily the Schwarzschild radius.

We discuss the elementary particle radius of black hole elementary particles before the birth of the universe. From section 6, the mass of ordinary matter before the birth of the universe increases, and its mass at the time of the birth of the universe is approximately $80 \%$ of the current mass. Since it is before the birth of the universe, there is no spacetime and only matter. From these, we conclude that the black hole elementary particle existed before the birth of the universe.

In conclusion, the elementary particle radius $r_{0}$ of the black hole elementary particle before the birth of the universe is $r_{0}=\frac{\Delta r}{2}$. First, the black hole elementary particle before the birth of the universe is different from that after the birth of the universe, and it is made not in the state with spacetime but in the state without spacetime. Second, since there is no spacetime, gravity does not work and there are no spacetime changes and singularities. Third, all ordinary elementary particle radii $r_{0}$ are $r_{0}=\frac{\Delta r}{2}$. Therefore, we conclude that 
the elementary particle radius $r_{0}$ of the black hole elementary particle before the birth of the universe is $r_{0}=\frac{\Delta r}{2}$.

In summary, the radius of black hole elementary particles in each case is expressed as follows.

- Before the birth of the universe:

$$
r_{0}=\frac{\Delta r}{2}
$$

- After the birth of the universe:

$$
\begin{gathered}
r_{0}^{\prime}=r_{s}^{\prime}=r_{s} \sqrt{1-\frac{r_{s}}{r}} \\
r_{0}=r_{s} \\
r^{\prime}=\left(\left(N-\frac{1}{2}\right) \Delta r+r_{s}\right) \sqrt{1-\frac{r_{s}}{r}} \\
r=\left(N-\frac{1}{2}\right) \Delta r+r_{s}
\end{gathered}
$$

\subsection{Minimum Mass and Minimum Radius of Black Hole Elementary Particles After the Birth of the Universe}

We show the minimum mass and minimum radius of black hole elementary particles after the birth of the universe.

The minimum mass of black hole elementary particles after the birth of the universe is the mass that satisfies the following equation.

$$
r_{0}=\frac{\Delta r}{2}=r_{s}
$$

First, the elementary particle radius after the birth of the universe is $r_{0}=\frac{\Delta r}{2}$ if $\frac{\Delta r}{2} \geqq r_{s}$, and $r_{0}=r_{s}$ if $\frac{\Delta r}{2}<r_{s}$.

Second, the elementary particle radius $r_{0}$ of black hole elementary particles after the birth of the universe is $r_{0}=r_{s}$. That is, when $r_{0}=r_{s}$, elementary particles are black hole elementary particles. 
Third, the minimum radius $\frac{\Delta r}{2}$ is expressed as $\frac{\Delta r}{2}=\frac{\mathrm{k}_{\mathrm{s}} T_{s p}}{2 M}$ and becomes smaller as the mass increases. The Schwarzschild radius $r_{s}$ is expressed as $r_{s}=\frac{2 \mathrm{G} M}{\mathrm{c}^{2}}$ and becomes larger as the mass increases.

Therefore, that minimum mass is the mass that satisfies equation (11.21). Specifically, the minimum mass $M_{b}$ of black hole elementary particles after the birth of the universe is expressed as

$$
M_{b}=\mathrm{m}_{\mathrm{p}} \sqrt{\frac{s}{2}},
$$

where $m_{p}$ is the Planck mass and is expressed as

$$
m_{p}=\sqrt{\frac{\hbar c}{G}} .
$$

The minimum radius of black hole elementary particles after the birth of the universe is the radius at which $r_{0}=\frac{\Delta r}{2}=r_{s}$. That is, the radius of the elementary particle at the mass $M_{b}$ is that minimum radius. Specifically, the minimum radius $r_{0 b}$ of black hole elementary particles after the birth of the universe is expressed as

$$
r_{0 b}=\mathrm{l}_{\mathrm{p}} \sqrt{2 s},
$$

where $l_{p}$ is the Planck length and is expressed as

$$
l_{p}=\sqrt{\frac{\hbar G}{c^{3}}} .
$$

The only matter with the spin quantum number of $1 / 2$ can become black hole elementary particles, so substituting $s=1 / 2$ into $M_{b}$ and $r_{0 b}$, they are expressed as follows.

$$
\begin{gathered}
M_{b}=\mathrm{m}_{\mathrm{p}} \sqrt{\frac{s}{2}}=\frac{\mathrm{m}_{\mathrm{p}}}{2} \quad\left(s=\frac{1}{2}\right) . \\
r_{0 b}=\mathrm{l}_{\mathrm{p}} \sqrt{2 s}=\mathrm{l}_{\mathrm{p}} \quad\left(s=\frac{1}{2}\right) .
\end{gathered}
$$

From equations (11.26) and (11.27), the minimum mass of black hole elementary particles after the birth of the universe is half the Planck mass, the minimum radius of that is the Planck length. Therefore, the minimum radius of matter after the birth of the universe is the Planck length. Note that this calculation does not take into account spacetime changes.

In summary, ideally, the matter within black holes turns into a single elementary particle, the elementary particle radius of the black hole elementary particle after the birth of the universe is the Schwarzschild radius, and the black hole elementary particle rotates at the 
speed of light. Therefore, gravitons at the speed of light can be emitted from black holes, and there is no black hole that nothing can escape from it at least after the birth of the universe. In this way, problems in black holes such as singularity are solved.

\section{Spacetime Particles and Potential Spacetime Particles}

In this section, we discuss spacetime particles with kinetic energy and potential spacetime particles with potential energy. Spacetime particles are always produced from the center of matter, but the mass or energy of matter is not always consumed. For example, the rest mass of elementary particles does not decrease. Therefore, from the law of energy conservation, there must be energy to produce spacetime particles. To solve this problem, we assume potential spacetime particles with potential energy.

\subsection{Properties of Spacetime Particles}

From the contents so far, we explain the important properties of spacetime particles.

Spacetime particles have three sides. First, spacetime particles are particles that compose and quantize time, space, energy, and mass. Second, spacetime particles are gravitons carrying gravity. Third, spacetime particles are dark matter.

The difference between the space with gravitons and the space without gravitons is as follows.

In the space with gravitons, gravity and an inertial force work, and time and space can be quantized and counted.

In the space without gravitons, gravity and an inertial force do not work, time and space cannot be quantized and cannot be counted, and the entire space becomes one minimum spacetime. Therefore, even in the space without gravitons, there is spacetime, but the entire space becomes one minimum spacetime. For example, in the space outside the universe and in the space before the birth of the universe, it is not that there is no spacetime, and the entire space is one minimum spacetime as within the elementary particles.

The generally recognized spacetime is the space where gravitons exist. Therefore, the birth of the universe means that the space with gravitons was created in the minimum spacetime that extends infinitely. Note that spacetime means space with gravitons unless otherwise noted.

Spacetime particles are amorphous particles that change shape depending on the situation. First, considering from the gravity equation of thermodynamic gravity form, spacetime particles are the spherical surface particles, and the volume of spacetime particles is represented as $\Delta V=4 \pi r^{2} \times \Delta r(r=N \Delta r)$. From this, the shape of spacetime particles changes depending on radius $r$. Second, the square of the absolute value of a wave function represents the 
spacetime particle number density within an elementary particle. From this, the shape of spacetime particles within an elementary particle changes depending on the wave function. Therefore, we conclude that spacetime particles are amorphous particles that change shape depending on the situation.

Spacetime particles are copy particles that copy the mass and spin quantum number of matter. First, the dimensionless temperature $T_{s p}$ of spacetime particles is expressed as $T_{s p}=4 \pi s$, where $s$ is the spin quantum number of matter. Second, the quantized minimum distance $\Delta r$ is expressed as $\Delta r=\frac{\mathrm{k}_{\mathrm{sp}} T_{s p}}{M}$, where $M$ is the active gravitational mass of matter. Therefore, we conclude that spacetime particles are copy particles that copy the mass and spin quantum number of matter.

In this way, the important properties of spacetime particles are explained.

\subsection{Production of Spacetime Particles}

Starting from the first law of thermodynamics, we discuss the production of spacetime particles that satisfies the law of energy conservation.

First of all, we show the conditions to calculate the production energy of spacetime particles from the first law of thermodynamics.

First, from $N=\frac{t}{\Delta t}$, one spacetime particle is produced for each quantized minimum time $\Delta t$.

Second, from conclusion 6.1, since the expansion source of spacetime is at the center of individual matter with active gravitational mass, spacetime particles are produced at $r=\Delta r$.

Third, spacetime particles distributed around matter move away from the matter at the speed of light. That is, spacetime particles move a distance $\Delta r$ in time $\Delta t$ and move at a velocity $v=\frac{\Delta r}{\Delta t}=\mathrm{c}$.

Fourth, from the law of energy conservation, spacetime particles must be produced by consuming energy or mass.

Fifth, the rest energy or rest mass of elementary particles does not decrease.

Therefore, spacetime particles distributed around matter move from $r=0$ to $r=\Delta r$ in time $\Delta t$ at the speed of light of $v=\frac{\Delta r}{\Delta t}=\mathrm{c}$, and one spacetime particle is produced by consuming energy for each quantized minimum time $\Delta t$.

We calculate the production energy of spacetime particles from the first law of thermodynamics and these conditions.

The first law of thermodynamics in time $\Delta t$ is expressed as

$$
\Delta U=Q+W
$$

where $\Delta U$ is the amount of change in internal energy in time $\Delta t, Q$ is the quantity of energy supplied as heat from the outside in time $\Delta t, W$ is the amount of work done in time $\Delta t$. 
In the production of spacetime particles, assuming adiabatic changes, $Q$ is expressed as

$$
Q=0
$$

The amount $W$ of work is expressed as

$$
W=P \Delta V,
$$

where $P$ is the pressure of spacetime particles, and $\Delta V$ is the expansion volume of spacetime in $\Delta t$ time. From equations (12.2) and (12.3), $\Delta U$ is expressed as

$$
\Delta U=W=P \Delta V .
$$

Since pressure is usually outward, the increase in volume means that work was done from inside to outside, and work $W$ is negative. However, the pressure of spacetime particles at $r=\Delta r$ is inward because the force exerted from spacetime particles at $r=\Delta r$ is the gravitational force of attraction. For this reason, in the spacetime expansion, the increase in volume means that work was done from outside to inside. Therefore, work $W$ in the spacetime expansion is positive.

From equation (4.4), the volume $\Delta V$ can be expressed as $\Delta V=4 \pi r^{2} \times \Delta r$. From equations (2.2) and (2.3), the force $F$ by pressure can be expressed as $F=P \times 4 \pi r^{2}$. From these, equation (12.4) is expressed as

$$
\Delta U=W=P \Delta V=P \times 4 \pi r^{2} \times \Delta r=F \Delta r .
$$

Since one spacetime particle is produced for each quantized minimum time $\Delta t$, with respect to the mass $m_{s p}$ of one spacetime particle, the force $F$ is expressed as

$$
F=m_{s p} a
$$

where $a$ is the acceleration exerted to mass $m_{s p}$. We consider the acceleration $a$ not as the gravitational acceleration, but as the acceleration that moves a distance $\Delta r$ in time $\Delta t$ and accelerates the velocity from $v=0$ to $v=\frac{\Delta r}{\Delta t}=\mathrm{c}$. Then, the acceleration $a$ is expressed as

$$
a=\frac{\frac{\Delta r}{\Delta t}-0}{\Delta t}=\frac{\Delta r}{\Delta t^{2}}
$$

Therefore, from equations (12.6) and (12.7) and $\frac{\Delta r}{\Delta t}=\mathrm{c}, \Delta U$ is expressed as

$$
\Delta U=F \Delta r=m_{s p} a \times \Delta r=m_{s p} \times \frac{\Delta r^{2}}{\Delta t^{2}}=m_{s p} \mathrm{c}^{2}
$$

From the above, for every quantized minimum time $\Delta t$, the kinetic energy of $m_{s p} \mathrm{c}^{2}$ is supplied from outside, one spacetime particle is produced, and the spacetime radius expands 
by $\Delta r$. From this calculation, the speed of spacetime particles is not the speed of light from the beginning but is accelerated from speed $v=0$ to speed $v=c$ in the quantized minimum time $\Delta t$. As a result, spacetime particles have the speed of light. The kinetic energy $E$ of spacetime particles is calculated in this way and is expressed as $E=m_{s p} \mathrm{c}^{2}$.

We explain potential spacetime particles. In the calculation of the production energy of spacetime particles, we implicitly have assumed follows.

Assumption 12.1: There are a large number of particles with velocity 0 , kinetic energy 0 and mass $m_{s p}$ at $r=0$.

These particles have mass but no kinetic energy because they have zero velocity. To distinguish these particles from spacetime particles with kinetic energy, we call these particles potential spacetime particles.

Even if there are a large number of potential spacetime particles at the center of matter, we cannot observe them directly because they have no kinetic energy. Observable particles are particles with kinetic energy. Therefore, potential spacetime particles are virtual particles. The virtual particle here means a particle that cannot be directly observed.

From the kinetic energy of spacetime particles, we calculate the gravitational acceleration that generates kinetic energy.

Since spacetime particles and potential spacetime particles have no charge and only mass, they receive force only from gravitational fields. Therefore, the acceleration $a$ of the force that generates kinetic energy is the gravitational acceleration, and the kinetic energy of spacetime particles is generated by consuming the potential energy of gravity. From these, we calculate the gravitational acceleration.

Since spacetime particles without active gravitational mass are produced from potential spacetime particles, potential spacetime particles also do not have active gravitational mass. From equation (11.1), the gravitational acceleration $a$ of particles without active gravitational mass is expressed as

$$
a=-\frac{2 \mathrm{G} M}{r^{2}} .
$$

From equation (12.9), equation (12.8) is expressed as

$$
-m_{s p} \times \frac{2 \mathrm{G} M}{r^{2}} \times \Delta r=m_{s p} \mathrm{c}^{2} .
$$

The radius $r$ satisfying equation (12.10) is expressed as

$$
r=\mathrm{i} \sqrt{r_{s} \times \Delta r},
$$

where $i$ is the imaginary unit $(i=\sqrt{-1})$. From equation (12.11), equation (12.9) is expressed as 


$$
a=\frac{2 \mathrm{G} M}{r_{s} \times \Delta r},
$$

and equation (12.10) is expressed as

$$
m_{s p} \times \frac{2 \mathrm{G} M}{r_{s} \times \Delta r} \times \Delta r=m_{s p} \mathrm{c}^{2} .
$$

Therefore, when the mass $m_{s p}$ is moved from $r=0$ to $r=\Delta r$ by the gravitational acceleration of equation (12.12), kinetic energy is $m_{s p} \mathrm{c}^{2}$.

We derive the gravity of $F=\frac{2 \mathrm{GMm}}{r_{s} \times \Delta r}$. The gravity $F$ based on equation (12.12) is expressed as

$$
F=\frac{2 \mathrm{G} M m}{r_{s} \times \Delta r}
$$

Since the gravity of equation (12.14) does not normally exist, we derive that gravity by considering the spacetime change caused by gravity. When considering the spacetime change caused by gravity, gravity is expressed as equation (11.7). Equation (11.7) is the gravity received by the matter with active gravitational mass. The gravity received by spacetime particles without active gravitational mass is expressed as twice the gravity of equation (11.7), and it is expressed as

$$
F^{\prime}=-\frac{2 \mathrm{G} M m}{r^{\prime 2}}=-\frac{2 \mathrm{G} M m}{r^{2}\left(1-\frac{r_{s}}{r}\right)} .
$$

Substituting $r=\Delta r$ into equation (12.15), it is expressed as

$$
F^{\prime}=-\frac{2 \mathrm{G} M m}{\Delta r^{2}\left(1-\frac{r_{s}}{\Delta r}\right)} .
$$

Assuming $\Delta r<<r_{s}$, because of $1<<\frac{r_{s}}{\Delta r}$, equation (12.16) can be approximated as

$$
F \fallingdotseq \frac{2 \mathrm{G} M m}{r_{s} \times \Delta r}
$$

In this way, the gravity of $F=\frac{2 \mathrm{G} M m}{r_{s} \times \Delta r}$ is derived.

We discuss the matter that generates gravity of $F=\frac{2 \mathrm{GMm}}{r_{s} \times \Delta r}$.

First, from the derivation process, that matter is the black hole elementary particle with the elementary particle radius $r_{0}=\frac{\Delta r}{2}<<r_{s}$.

Second, from $\frac{\Delta r}{2}<<r_{s}$, that black hole elementary particle has a huge mass. If the mass $M$ is almost infinite, the elementary particle radius $r_{0}$ is expressed as 


$$
r_{0}=\frac{\Delta r}{2}=\frac{\mathrm{k}_{\mathrm{sp}} T_{s p}}{2 M} \fallingdotseq 0<<r_{s}
$$

Third, from the production energy of spacetime particles, in time $\Delta t$, potential spacetime particles move from $r=0$ to $r=\Delta r$, and their speed is accelerated from $v=0$ to $v=\frac{\Delta r}{\Delta t}=\mathrm{c}$, and spacetime particles are produced. For this reason, that black hole elementary particle exists at the center of $r=0$ within an elementary particle, and at the same time, there are a large number of potential spacetime particles at $r=0$.

Fourth, ordinary elementary particles do not have the radius of $r_{0}=\frac{\Delta r}{2}<<r_{s}$. From equation (11.8), the elementary particle radius $r_{0}$ of black hole elementary particles after the birth of the universe is $r_{0}=r_{s}$. For this reason, that black hole elementary particles are not ordinary black hole elementary particles.

Fifth, potential spacetime particles have mass, and there are a large number of potential spacetime particles at one point of $r=0$. This situation is the same as black holes. For this reason, that black hole elementary particles are composed of potential spacetime particles. We call it the potential black hole elementary particle.

Sixth, potential spacetime particles have no kinetic energy, so they cannot be observed. For this reason, in principle, potential spacetime particles do not receive gravity from ordinary matter and do not exert gravity to ordinary matter and receive only gravity from the same potential spacetime particles.

Seventh, the gravity of potential black hole elementary particles is carried by potential spacetime particles, and potential spacetime particles turn into spacetime particles at $r=\Delta r$. For this reason, the gravity of potential black hole elementary particles works only up to $r=\Delta r$. Moreover, the gravity of potential black hole elementary particles works only within the quantized minimum time $\Delta t$. That is, when viewed at the quantized minimum time $\Delta t$ unit, potential spacetime particles turn into spacetime particles, so the gravity of potential black hole elementary particles is not working.

More precisely, the gravity of potential black hole elementary particles works only in the space of $\Delta r_{p} \fallingdotseq 0 \leqq r<\Delta r$, where $\Delta r_{p}$ is the quantized minimum distance of potential black hole elementary particles and almost zero. Since the elementary particle radius $r_{0}$ of potential black hole elementary particles is $r_{0}=\frac{\Delta r_{p}}{2}$, potential spacetime particles in space exist only from $r=\Delta r_{p}$. For this reason, the gravity of potential black hole elementary particles works only from $\Delta r_{p} \fallingdotseq 0$.

In summary, the gravity $F_{p}^{\prime}$ of potential black hole elementary particles is expressed as follows.

- If $\Delta r \leqq r, F_{p}^{\prime}=0$.

- If $\Delta r_{p} \leqq r<\Delta r, F_{p}^{\prime}=\frac{2 \mathrm{G} M_{p} m_{s p}}{r_{s p} \times \Delta r}$, 
where $M_{p}$ and $r_{s p}$ are the mass and Schwarzschild radius of potential black hole elementary particles.

From these, we conclude that the matter generating gravity of $F=\frac{2 \mathrm{G} M m}{r_{s} \times \Delta r}$ is the potential black hole elementary particle.

We explain the law of energy conservation in the production of spacetime particles. Since there is the potential black hole elementary particle within an elementary particle, the potential black hole elementary particle is an external particle to the elementary particles. The external particle giving the energy of $E=m_{s p} c^{2}$ is the potential black hole elementary particle. The gravity of potential black hole elementary particles is a repulsive force. Potential spacetime particles are moved by the repulsive force, and spacetime particles are produced. That is, the spacetime particle with kinetic energy is produced from the potential spacetime particle with the potential energy of gravity.

We calculate the kinetic energy of spacetime particles and the potential energy of potential spacetime particles. We refer to the observer's reference frame as the $t$ frame and the reference frame of potential spacetime particles as the $t^{\prime}$ frame. From the physical quantities observed from the different reference frames in section 8, the potential energy $U^{\prime}$ of the $t^{\prime}$ frame observed from the $t$ frame is expressed as

$$
U^{\prime}=-\int_{\infty}^{r} F^{\prime} \mathrm{d} r
$$

where $F^{\prime}$ is the gravity of the $t^{\prime}$ frame observed from the $t$ frame. From equation (12.19) and $F_{p}^{\prime}=0(\Delta r \leqq r)$, the potential energy $U^{\prime}$ of potential spacetime particles at $r=\Delta r$ is zero.

$$
U^{\prime}=-\int_{\infty}^{\Delta r} F_{p}^{\prime} \mathrm{d} r=0 .
$$

From equations (12.19) and (12.20), the potential energy $U^{\prime}$ of potential spacetime particles at $r=\Delta r_{p} \fallingdotseq 0$ is expressed as

$$
U^{\prime}=-\int_{\infty}^{0} F_{p}^{\prime} \mathrm{d} r=\int_{0}^{\Delta r} F_{p}^{\prime} \mathrm{d} r=F_{p}^{\prime} \Delta r=m_{s p} \mathrm{c}^{2} .
$$

The velocity $v$ of spacetime particles is $v=0$ when $\Delta r_{p} \fallingdotseq 0 \leqq r<\Delta r$ and $v=\mathrm{c}$ when $\Delta r \leqq r$. For this reason, the kinetic energy $E$ of spacetime particles is $E=0$ when $\Delta r_{p} \fallingdotseq 0 \leqq r<\Delta r$ and $E=m_{s p} c^{2}$ when $\Delta r \leqq r$. In summary, energy relations are expressed as follows.

- In the space of $\Delta r_{p} \fallingdotseq 0 \leqq r<\Delta r$ :

$$
F_{p}^{\prime}=\frac{2 \mathrm{G} M_{p} m_{s p}}{r_{s p} \times \Delta r}
$$




$$
\begin{gathered}
U^{\prime}=m_{s p} \mathrm{c}^{2} \\
E=0
\end{gathered}
$$

- In the space of $\Delta r \leqq r$ :

$$
\begin{gathered}
F_{p}^{\prime}=0 \\
U^{\prime}=0 \\
E=m_{s p} \mathrm{c}^{2}
\end{gathered}
$$

Therefore, the following law of energy conservation holds.

$$
E+U^{\prime}=m_{s p} \mathrm{c}^{2} .
$$

The potential energy of $U^{\prime}=m_{s p} c^{2}$ is converted into the kinetic energy of $E=m_{s p} \mathrm{c}^{2}$, and the potential spacetime particle turns into the spacetime particle. Potential spacetime particles have the potential energy of $U^{\prime}=m_{s p} \mathrm{c}^{2}$, and spacetime particles have the kinetic energy of $E=m_{s p} c^{2}$. In this way, the law of energy conservation holds in the production of spacetime particles.

In summary, it is as follows. There are potential spacetime particles with the potential energy of $U^{\prime}=m_{s p} c^{2}$ and spacetime particles with the kinetic energy of $E=m_{s p} c^{2}$. The potential black hole elementary particle is composed of potential spacetime particles, it exists at the center of $r=0$ within an elementary particle. The potential energy of $U^{\prime}=m_{s p} c^{2}$ is converted into the kinetic energy of $E=m_{s p} \mathrm{c}^{2}$, and the potential spacetime particle turns into the spacetime particle. In this way, the law of energy conservation holds in the production of spacetime particles.

\subsection{Where Potential Black Hole Elementary Particles Exist}

We discuss where potential black hole elementary particles exist.

First, there are potential blackhole elementary particles within elementary particles with active gravitational mass, and there are no potential blackhole elementary particles within elementary particles without active gravitational mass. This is because potential black hole elementary particles produce spacetime particles around elementary particles. 
Second, from the production energy of spacetime particles, potential black hole elementary particles exist at the center of $r=0$ within elementary particles with active gravitational mass.

Third, potential black hole elementary particles exist inside charged particles. The elementary particle radius $r_{0 p}$ of potential black hole elementary particles is $r_{0 p}=\frac{\Delta r_{p}}{2} \fallingdotseq 0$, and the radius $r_{0 c}$ of charged particles is $r_{0 c}=\frac{\Delta r_{i n}}{2}$. Since the mass of potential black hole elementary particles is huge, $r_{0 p}$ is usually much smaller than $r_{0 c}$. There are charged particles and the potential black hole elementary particle at the center of elementary particles. Therefore, we conclude that potential black hole elementary particles exist inside charged particles. However, charged particles before the birth of the universe are excluded.

Fourth, when a particle annihilates with an antiparticle, potential black hole elementary particles exist in a vacuum with charged particles. At this time, an elementary particle is decomposed into spacetime particles and charged particles, spacetime particles are emitted as photons, and charged particles exist in a vacuum. Since photons have no active gravitational mass, there is no potential black hole elementary particle within photons. Since charged particles undergo pair production and annihilation in a vacuum, they require kinetic energy. However, charged particles have only charges and no energy. Moreover, that energy exists only within the quantized minimum time $\Delta t$, and only potential black hole elementary particles can have such energy. Therefore, at this time, we conclude that potential black hole elementary particles exist in the vacuum together with charged particles.

From these, potential black hole elementary particles always exist together with charged particles. Since spacetime particles are produced in each elementary particle, there is one potential black hole elementary particle within at least one elementary particle with active gravitational mass. The internal structure of elementary particles is as follows.

- In the space of $r \leqq \frac{\Delta r_{p}}{2}$, the potential black hole elementary particle is distributed.

- In the space of $\frac{\Delta r_{p}}{2}<r \leqq \frac{\Delta r_{i n}}{2}$, charged particles are distributed.

- In the space of $\frac{\Delta r_{i n}}{2}<r \leqq \frac{\Delta r}{2}$, spacetime particles are distributed.

\subsection{Spacetime and Production of Spacetime Particles Before the Birth of the Universe}

We discuss the spacetime and production of spacetime particles before the birth of the universe. From sections 6 and 11, before the birth of the universe, there is no spacetime, and only the mass of ordinary matter increases, so only the black hole elementary particle with $r_{0}=\frac{\Delta r}{2}$ exists. Spacetime particles return by the gravity of the black hole with $r_{0}=\frac{\Delta r}{2}$. As a result, before the birth of the universe, spacetime particles are produced within the black hole elementary particle. We discuss these in detail. 
We explain the production of spacetime particles within elementary particles. The production energy of spacetime particles is calculated as $E=m_{s p} a \times \Delta r=m_{s p} \mathrm{c}^{2}$. For this reason, potential spacetime particles need to be moved by a distance of $\Delta r$ to produce spacetime particles. For example, the spacetime particle at $r=\Delta r$ is produced by moving the potential spacetime particle from $r=\frac{\Delta r_{p}}{2} \fallingdotseq 0$ to $r=\Delta r+\frac{\Delta r_{p}}{2} \fallingdotseq \Delta r$. The inside of elementary particles is represented as $r \leqq \frac{\Delta r}{2}$. Therefore, we conclude that spacetime particles within $r \leqq \frac{\Delta r}{2}$ are produced by moving potential spacetime particles from $r=\Delta r+\frac{\Delta r_{p}}{2} \fallingdotseq \Delta r$ to $r=\frac{\Delta r_{p}}{2} \fallingdotseq 0$.

We explain the existed particles before the birth of the universe.

The first is one black hole elementary particle. From section 6, the mass of ordinary matter before the birth of the universe increases, and its mass at the time of the birth of the universe is approximately $80 \%$ of the current mass. Since it is before the birth of the universe, there is no spacetime and there is only matter in one minimum spacetime. Therefore, one black hole elementary particle existed even before the birth of the universe, and its mass was increasing.

The second is potential black hole elementary particles. This is because potential black hole elementary particles are required to increase the mass.

The third is charged particles. This is because potential black hole elementary particles exist together with charged particles. However, when charged particles and anti-charged particles exist in pairs, they annihilate, so only charged particles existed.

These mean that there is one black hole elementary particle in the space without gravitons, and there are charged particles and potential black hole elementary particles within the black hole elementary particle.

Information about these particles is shown below.

$$
r_{0 p}=\frac{\Delta r_{p}}{2}<<r_{s p}
$$

where $r_{0 p}, \Delta r_{p}$ and $r_{s p}$ are the elementary particle radius, quantized minimum distance and Schwarzschild radius of potential black hole elementary particles.

$$
r_{0}=\frac{\Delta r}{2}<<r_{s}
$$

where $r_{0}, \Delta r$ and $r_{s}$ are the elementary particle radius, quantized minimum distance and Schwarzschild radius of the black hole elementary particle.

$$
M \leqq M_{p}
$$

where $M$ is the mass of the black hole elementary particle, and $M_{p}$ is the mass of potential black hole elementary particles.

$$
\frac{\Delta r_{p}}{2} \leqq \frac{\Delta r}{2}<r_{s} \leqq r_{s p}
$$


We discuss the production of spacetime particles within the black hole elementary particle before the birth of the universe. To produce spacetime particles within elementary particles, potential spacetime particles need to move as follows.

$$
r=\frac{\Delta r_{p}}{2} \rightarrow r=\Delta r+\frac{\Delta r_{p}}{2} \rightarrow r=\frac{\Delta r_{p}}{2}
$$

Spacetime particles return to the black hole elementary particle again by the gravity of the black hole elementary particle. Therefore, time goes back to the past. The kinetic energy $E$ and potential energy $U$ in this process are shown as follows.

- Case1: $r=\frac{\Delta r_{p}}{2}, U^{\prime}=m_{s p} c^{2}, E=0$

- Case2: $r=\Delta r+\frac{\Delta r_{p}}{2}, U^{\prime}=0, E=m_{s p} c^{2}$

- Case3: $r=\Delta r+\frac{\Delta r_{p}}{2}, U^{\prime}=0, E=0$

- Case4: $r=\frac{\Delta r_{p}}{2}, U^{\prime}=-m_{s p} \mathrm{c}^{2}, E=m_{s p} \mathrm{c}^{2}$

At this time, in principle, spacetime particles receive only the gravity of the black hole elementary particle, and potential spacetime particles receive only the gravity of the potential black hole elementary particle. Therefore, this process is explained as follows.

In case1, the potential spacetime particle is emitted by the repulsive force of the potential black hole elementary particle.

In case2, the potential spacetime particle obtains kinetic energy and turns into the spacetime particle.

In case3, the spacetime particle loses kinetic energy by the attractive force of the black hole elementary particle and turns into the potential spacetime particle.

In case4, the potential spacetime particle is returned by the attractive force of the potential black hole elementary particle.

Before the birth of the universe, spacetime particles within the black hole elementary particle were produced in this way.

We discuss spacetime before the birth of the universe.

First, from the production of spacetime particles, we discuss spacetime before the birth of the universe. Since the production of spacetime particles mentioned earlier is repeated, time $t$ and radius $r$ and the kinetic energy at $r=\Delta r$ and the kinetic energy within $r \leqq \frac{\Delta r}{2}$ change as table 1 .

Table 1. Changes in spacetime and kinetic energy before the birth of the universe. 


\begin{tabular}{|c|c|c|c|}
\hline Time & Distance & Kinetic Energy at $r=\Delta r$ & Kinetic Energy within $r \leqq \frac{\Delta r}{2}$ \\
\hline \hline 0 & 0 & 0 & $N \times m_{s p} \mathrm{c}^{2}$ \\
\hline$\rightarrow \Delta t$ & $\Delta r$ & $m_{s p} \mathrm{c}^{2}$ & $N \times m_{s p} \mathrm{c}^{2}$ \\
\hline$\rightarrow 0$ & 0 & 0 & $(N+1) \times m_{s p} \mathrm{c}^{2}$ \\
\hline$\rightarrow \Delta t$ & $\Delta r$ & $m_{s p} \mathrm{c}^{2}$ & $(N+1) \times m_{s p} \mathrm{c}^{2}$ \\
\hline$\rightarrow 0$ & 0 & 0 & $(N+2) \times m_{s p} \mathrm{c}^{2}$ \\
\hline$\rightarrow \Delta t$ & $\Delta r$ & $m_{s p} \mathrm{c}^{2}$ & $(N+3) \times m_{s p} \mathrm{c}^{2}$ \\
\hline$\rightarrow 0$ & 0 & 0 & \\
\hline$\rightarrow \ldots$ & & & \\
\hline
\end{tabular}

In this way, before the birth of the universe, spacetime is repeatedly created and annihilated. Spacetime particles are absorbed by the black hole elementary particle and spacetime disappears, at which time the mass of the black hole elementary particle increases. This means that the potential black hole elementary particle emits spacetime particles, and the black hole elementary particle absorbs them by gravity.

Second, from the spacetime change caused by gravity, we discuss spacetime before the birth of the universe. When we consider the spacetime change caused by gravity, the distance $r^{\prime}$ and the time $t^{\prime}$ are expressed as follows from equation (9.42).

$$
\begin{aligned}
r^{\prime} & =r \sqrt{1-\frac{r_{s}}{r}} . \\
t^{\prime} & =t \sqrt{1-\frac{r_{s}}{r}} .
\end{aligned}
$$

From $\frac{\Delta r}{2}<<r_{s}$ and $r=\Delta r$ and $t=\frac{r}{\mathrm{c}}$, equations (12.27) and (12.28) are expressed as follows.

$$
\begin{aligned}
& r^{\prime}=\Delta r \sqrt{1-\frac{r_{s}}{\Delta r}}=\mathrm{i} \sqrt{r_{s} \times \Delta r} . \\
& t^{\prime}=\Delta t \sqrt{1-\frac{r_{s}}{\Delta r}}=\mathrm{i} \sqrt{t_{s} \times \Delta t},
\end{aligned}
$$

where $t_{s}$ is expressed as

$$
t_{s}=\frac{r_{s}}{\mathrm{c}}
$$

Therefore, spacetime before the birth of the universe becomes imaginary spacetime. However, since imaginary spacetime disappears as soon as it is created and it cannot be observed, there is virtually no spacetime before the birth of the universe. 
We discuss the time before the birth of the universe. We set $N$ as the number of times that time $t$ has become $t=\Delta t$. Then, we can consider that time $t$ before the birth of the universe is expressed as

$$
t=N \Delta t
$$

The time before the birth of the universe in section 6 is the time $t$ when we think in this way. The total time before the birth of the universe is approximately 14.53 billion years from the calculation under the acceleration period matching condition in Section 6 .

Note that the actual time before the birth of the universe is expressed as $t^{\prime}=\mathrm{i} N \sqrt{t_{s} \times \Delta t}$ from equation (12.30). That is, the actual time before the birth of the universe is an imaginary time. Therefore, before the birth of the universe, the time of approximately 14.53 billion years cannot actually be observed and does not exist.

In summary, it is as follows. Before the birth of the universe, there was one black hole elementary particle in the space without gravitons, and there were charged particles and potential black hole elementary particles within the black hole elementary particle. The potential black hole elementary particle emits spacetime particles, the black hole elementary particle absorbs them by gravity, and its mass increases. For this reason, before the birth of the universe, spacetime was repeatedly created and annihilated, and spacetime was imaginary spacetime.

\subsection{Spacetime When Matter and Antimatter Undergo Pair Production and Annihilation}

We discuss spacetime when matter and antimatter undergo pair production and annihilation within the quantized minimum time $\Delta t$.

First, charged particles and spacetime particles are required even for pair production within the quantized minimum time $\Delta t$.

Second, from the uncertainty principle of $\Delta E \times \Delta t=\frac{\hbar}{2}$, the energy of the particle produced only within the quantized minimum time $\Delta t$ is the energy $\Delta E$ of the uncertainty principle.

Third, from equation (4.19), the energy $\Delta E$ of the uncertainty principle is the energy of spacetime particles.

Fourth, spacetime particles are produced by potential black hole elementary particles.

Fifth, since pair production and annihilation are repeated within the quantized minimum time $\Delta t$, spacetime particles within the produced particle or on spacetime do not increase.

Sixth, there is the quantized minimum time $\Delta t$ on spacetime and the energy $\Delta E$ within the produced particle, both of which are composed of spacetime particles. 
From these, we conclude as follows. Potential black hole elementary particles exist together with charged particles, and spacetime particles are emitted from potential black hole elementary particles. The spacetime particle has the energy $\Delta E$ of the uncertainty principle. From charged particles and the energy $\Delta E$ of the spacetime particle, matter and antimatter undergo pair production only within the quantized minimum time $\Delta t$. Matter and antimatter undergo annihilation, and the energy $\Delta E$ within the produced particle changes to the time $\Delta t$ of the spacetime particle on spacetime. The spacetime particle on spacetime undergoes annihilation, and it changes to the energy $\Delta E$ within the produced particle. In this way, one spacetime particle changes to the time $\Delta t$ of the spacetime particle on spacetime or the energy $\Delta E$ within the produced particle. Therefore, in pair production and annihilation of matter and antimatter, at the same time, spacetime is also repeatedly created and annihilated.

The summary of this section is as follows.

In general, the space with spacetime is the space where gravitons exist, the space without spacetime is the space where gravitons do not exist. In the space without gravitons, gravity and an inertial force do not work, time and space cannot be quantized and cannot be counted, and the entire space becomes one minimum spacetime. Therefore, even in the space without gravitons, there is spacetime, but the entire space becomes one minimum spacetime.

There are potential spacetime particles with the potential energy of $U^{\prime}=m_{s p} \mathrm{c}^{2}$ and spacetime particles with the kinetic energy of $E=m_{s p} c^{2}$. Since potential spacetime particles have no kinetic energy, they are virtual particles that cannot be observed directly. The potential black hole elementary particle is composed of potential spacetime particles, it exists at the center of $r=0$ within an elementary particle. The potential energy of $U^{\prime}=m_{s p} \mathrm{c}^{2}$ is converted into the kinetic energy of $E=m_{s p} c^{2}$, and the potential spacetime particle turns into the spacetime particle. In this way, the law of energy conservation holds in the production of spacetime particles.

Before the birth of the universe, there was one black hole elementary particle with the minimum radius $\frac{\Delta r}{2}$ in the space without gravitons, and there were charged particles and potential black hole elementary particles within the black hole elementary particle. The potential black hole elementary particle emits spacetime particles, the black hole elementary particle absorbs them by gravity, and its mass increases. For this reason, before the birth of the universe, spacetime was repeatedly created and annihilated, and spacetime was imaginary spacetime.

\section{Inflation}

In this section, we discuss inflation just the birth of the universe. Inflation is a phenomenon in which spacetime expands at a speed faster than the speed of light. From section 12, 
spacetime before the birth of the universe was the imaginary spacetime by one black hole, and spacetime was repeatedly created and annihilated. We discuss what happened from this state.

\subsection{Discussion of Inflation}

We discuss inflation just after the birth of the universe.

First of all, we show the initial value of the universe when inflation occurs. From the calculation of the acceleration period matching condition in section 6 , the initial value of the universe is shown as follows.

$$
\begin{gathered}
T_{s p}=4 \pi s=2 \pi \quad\left(s=\frac{1}{2}\right) . \\
M=M_{t} \times 0.762,
\end{gathered}
$$

where $M$ is the total mass of ordinary matter at the time of the birth of the universe, $M_{t}$ is the total mass of the current ordinary matter.

$$
\begin{gathered}
N=1 . \\
r=\Delta r=\frac{\mathrm{k}_{\mathrm{sp}} T_{s p}}{M} .
\end{gathered}
$$

These initial values are not only the state of the universe when inflation occurs, but also the state of the universe when the universe was born. From section 12, spacetime before the birth of the universe was the imaginary spacetime by one black hole, and spacetime was repeatedly created and annihilated. Therefore, in the universe at this time, there was only the black hole elementary particle with the elementary particle radius $r_{0}=\frac{\Delta r}{2}<r_{s}$, and spacetime was extremely small.

We show a repulsive force that causes the black hole elementary particle to collapse. The current universe is not in the state of one black hole elementary particle. Therefore, a repulsive force is required to collapse the black hole elementary particle. For particles without active gravitational mass, the gravity $F^{\prime}$ of the black hole elementary particle at $r=\Delta r$ is expressed as equation (12.17). For particles with active gravitational mass, from half of equation (12.17), the gravity $F^{\prime}$ of the black hole elementary particle at $r=\Delta r$ is expressed as

$$
F^{\prime}=\frac{\mathrm{G} M m}{r_{s} \times \Delta r}
$$


Therefore, at the birth of the universe, the gravity $F^{\prime}$ of the black hole elementary particle is a repulsive force. That repulsive force caused the black hole elementary particle to collapse. The gravity of spacetime particles works in the direction opposite to the direction in which gravitons move, and the gravity of potential spacetime particles works in the direction in which gravitons move. For this reason, if the gravity of the black hole elementary particle is a repulsive force, time goes back to the past.

We discuss the timing when inflation occurs. From section 12, spacetime before the birth of the universe was the imaginary spacetime by one black hole, and spacetime was repeatedly created and annihilated. The problem is what happened from this state. The mass of the black hole elementary particle is the mass originally possessed by the potential black hole elementary particle. For this reason, the mass of the potential black hole elementary particle decreases, and the mass of the black hole elementary particle increases. Therefore, that timing is when the mass of the black hole elementary particle becomes larger than the mass of the potential black hole elementary particle.

First, spacetime particles are produced at $r=\frac{\Delta r_{p}}{2}+\Delta r$ in real coordinates, where $\Delta r_{p}$ is the quantized minimum distance of potential black hole elementary particle and $\Delta r$ is the quantized minimum distance of black hole elementary particle.

Second, $\frac{\Delta r}{2}<r \leqq \frac{3 \Delta r}{2}$ in real coordinates corresponds to $r=\Delta r$ in graviton coordinates. $\frac{3 \Delta r}{2}<r \leqq \frac{5 \Delta r}{2}$ in real coordinates corresponds to $r=2 \Delta r$ in graviton coordinates.

Third, spacetime particles before the birth of the universe are inverted as soon as they are produced, and time goes back to the past.

Fourth, when spacetime particles are inverted, spacetime particles turn into potential spacetime particles.

Fifth, spacetime particles are produced by moving by $\Delta r$.

Sixth, the gravity of spacetime particles works in the direction opposite to the direction in which gravitons move.

Seventh, potential spacetime particles that invert from $r=\Delta r$ turn into spacetime particles within the black hole elementary particle. At this time, since there are no spacetime particles on spacetime, the repulsive force of the black hole elementary particle does not work. Potential spacetime particles that invert from $r=2 \Delta r$ turn into spacetime particles at $r=\Delta r$. At this time, since there is the spacetime particle on spacetime, the repulsive force of the black hole elementary particle works.

Eighth, when the mass $M$ of the black hole elementary particle is smaller than the mass $M_{p}$ of the potential black hole elementary particle $\left(M \leqq M_{p}\right.$ ), spacetime particles are produced at $r=\Delta r$ in graviton coordinates because of $\Delta r \geqq \Delta r_{p}$ and $\frac{\Delta r}{2}<\frac{\Delta r_{p}}{2}+\Delta r \leqq \frac{3 \Delta r}{2}$. When the mass $M$ of the black hole elementary particle is $M>M_{p}$, spacetime particles are produced at $r=2 \Delta r$ in graviton coordinates because of $\Delta r<\Delta r_{p}$ and $\frac{3 \Delta r}{2}<\frac{\Delta r_{p}}{2}+\Delta r \leqq \frac{5 \Delta r}{2}$.

Therefore, when the mass $M$ of the black hole elementary particle is $M>M_{p}$, the spacetime particle is produced at $r=2 \Delta r$ and turns into the potential spacetime particle. 
That potential spacetime particle moves to $r=\Delta r$ from $r=2 \Delta r$ and turns into the spacetime particle at $r=\Delta r$. At this time, the repulsive force of equation (13.5) works on the black hole elementary particle, and the black hole elementary particle collapses. The time $t=\Delta t$ at the birth of the universe is the time when the time returns from $t=2 \Delta t$ to $t=\Delta t$. More precisely, from equation (12.30), this time is an imaginary time.

From this argument, the original mass $M_{p 0}$ of potential black hole elementary particles is twice the total mass $M$ of ordinary matter at the birth of the universe.

$$
M_{p 0}=2 M .
$$

Therefore, the mass of potential black hole elementary particles is not infinite.

We explain the cause of faster-than-light inflation. The black hole elementary particle collapses by the repulsive force, and individual matter is produced. The elementary particle radius of the individual matter is much larger than the elementary particle radius of the black hole elementary particle with the total mass of ordinary matter. The change of the elementary particle radius to $\frac{\Delta r}{2}$ occurs at a speed faster than the speed of light by wave function reduction. In the case of inflation, the elementary particle radius expands to $\frac{\Delta r}{2}$, so that change is wave function enlargement. Therefore, inflation can be explained to be caused by the expansion of the elementary particle radius of the individual matter faster than the speed of light by wave function enlargement.

We show the expansion speed of the spacetime radius and the inter-matter radius in inflation. From equations (5.1) and (4.3), the spacetime radius $r_{\max }$ when $\frac{\Delta r}{2} \geqq r_{s}$ is expressed as

$$
r_{\max }=N \Delta r=2 N r_{0}\left(\frac{\Delta r}{2} \geqq r_{s}\right) .
$$

The expansion speed $v_{\max }$ of the spacetime radius $r_{\max }$ is expressed as

$$
v_{\max }=\frac{\mathrm{d} r_{\max }}{\mathrm{d} t}=2\left(N \times \frac{\mathrm{d} r_{0}}{\mathrm{~d} t}+r_{0} \times \frac{\mathrm{d} N}{\mathrm{~d} t}\right) .
$$

Inflation is caused by the expansion of the elementary particle radius, so $\frac{\mathrm{d} r_{0}}{\mathrm{~d} t} \neq 0$. From equations (8.3) and (8.4), $\frac{\mathrm{d} N}{\mathrm{~d} t}$ is expressed as

$$
\frac{\mathrm{d} N}{\mathrm{~d} t}=\frac{1}{\Delta t}
$$

From equation (13.9) and $r_{0}=\frac{\Delta r}{2}$ and $\mathrm{c}=\frac{\Delta r}{\Delta t}$, the expansion speed $v_{\text {max }}$ is expressed as

$$
v_{\max }=2 N \times \frac{\mathrm{d} r_{0}}{\mathrm{~d} t}+\mathrm{c}
$$


In inflation, the expansion speed of the elementary particle radius is much faster than the speed of light, so the following relationship holds.

$$
2 N \times \frac{\mathrm{d} r_{0}}{\mathrm{~d} t}>>\mathrm{c} .
$$

From equation (13.11), the expansion speed $v_{\max }$ can be approximated as

$$
v_{\max } \fallingdotseq 2 N \times \frac{\mathrm{d} r_{0}}{\mathrm{~d} t}
$$

From equations (6.29) and (13.7) and (13.12), the Hubble parameter $H$ is expressed as

$$
H=\frac{v_{\max }}{r_{\max }}=\frac{\left(\frac{\mathrm{d} r_{0}}{\mathrm{~d} t}\right)}{r_{0}} .
$$

From equations (6.28) and (13.13), the expansion speed $v$ of the inter-matter radius $r$ is expressed as

$$
v=r H=r \times \frac{\left(\frac{\mathrm{d} r_{0}}{\mathrm{~d} t}\right)}{r_{0}} .
$$

At the birth of the universe, the inter-matter radius $r$ is nearly zero. This means that the distance between matter is nearly 0 and matter is densely packed. The minimum inter-matter radius is the elementary particle radius $r_{0}$. The matter at this time is in contact with each other at the elementary particle radius $r_{0}$, as in the case of atomic nuclei. Therefore, from equation (5.49), the inter-matter radius $r$ in inflation is expressed as

$$
r \fallingdotseq N_{e r}^{\frac{1}{3}} \times r_{0}
$$

where $N_{e r}$ is the number of elementary particles within the inter-matter radius $r$. From equations (13.14) and (13.15), the expansion speed $v$ is expressed as

$$
v \fallingdotseq N_{e r}^{\frac{1}{3}} \times \frac{\mathrm{d} r_{0}}{\mathrm{~d} t}
$$

In summary, the radius and the expansion speed are expressed as follows.

$$
\begin{gathered}
r_{\max }=2 N r_{0} . \\
r \fallingdotseq N_{e r}^{\frac{1}{3}} \times r_{0} . \\
v_{\max } \fallingdotseq 2 N \times \frac{\mathrm{d} r_{0}}{\mathrm{~d} t} .
\end{gathered}
$$




$$
v \fallingdotseq N_{e r}^{\frac{1}{3}} \times \frac{\mathrm{d} r_{0}}{\mathrm{~d} t}
$$

In inflation, $2 N<<N_{e r}^{\frac{1}{3}}$, so the following relationship holds.

$$
\begin{aligned}
& r_{\max }<<r . \\
& v_{\max }<<v .
\end{aligned}
$$

Therefore, the unobservable inter-matter radius $r$ is much larger than the observable spacetime radius $r_{\max }$. That is, the universe ( $=$ inter-matter radius $r$ ) is much larger than the universe ( = spacetime radius $\left.r_{\max }\right)$ we can observe.

Inflation is explained in this way.

\subsection{Calculation of Inflation}

We calculate the radius expansion rate and expansion speed in inflation.

First of all, we show the premises in the calculation of inflation.

Premise 13.1: At the time of inflation, the mass $M$ of the black hole elementary particle is divided into each mass $M_{1}$ of equation (7.36).

Premise 13.2: The number divided the total mass of ordinary matter by the mass $M_{1}$ of equation (7.36) is the number of expansion sources at that time.

From section 7 , if we average over the universe, the mass $M_{1}$ of ordinary matter, the mass $M_{2}$ of dark matter and the mass $M_{3}$ of dark energy exist for each sphere volume with the radius $r_{A}$. From this, we conclude premises 13.1 and 13.2. Note that the elementary particles with mass $M_{1}$ are black hole elementary particles.

Premise 13.3: The total mass $M_{t}$ of the current ordinary matter is $3.00 \times 10^{52} \mathrm{~kg}$.

From the mass of the observable universe [18], we conclude premise 13.3.

Premise 13.4: The elementary particle radius $r_{01}$ of matter with the mass $M_{1}$ is the Schwarzschild radius $r_{s}$.

Since the mass $M_{1}$ is large, the Schwarzschild radius $r_{s}$ is larger than the minimum radius $\frac{\Delta r}{2}$. For this reason, we conclude premise 13.4 .

Premise 13.5: Spacetime at the birth of the universe is an imaginary spacetime, but we set radius and time as their absolute values.

Premise 13.6: The spacetime particle number $N$ before inflation is one, and the spacetime particle number $N$ after inflation is two.

Premise 13.7: In wave function enlargement, the mass $M$ of equation (8.75) is the total mass $M_{t}$ of the current ordinary matter. 
From the above premises, we calculate the radius expansion rate and expansion speed in inflation.

From these premises and the contents so far, we show each physical quantity associated with the radius expansion rate in inflation.

From premise 13.3, the total mass $M_{t}$ of the current ordinary matter is expressed as

$$
M_{t}=3.00 \times 10^{52} \mathrm{~kg} .
$$

From equations (13.2) and (13.19), the mass $M$ of the black hole elementary particle at the birth of the universe is expressed as

$$
M=2.29 \times 10^{52} \mathrm{~kg} .
$$

From equation (7.36), the mass $M_{1}$ of the ordinary matter of expansion source is expressed as

$$
M_{1}=7.76 \times 10^{31} \mathrm{~kg} .
$$

From premise 13.2, the number $N_{e 1}$ of expansion sources at inflation is expressed as

$$
N_{e 1}=\frac{M}{M_{1}} .
$$

From equation (13.15), the maximum inter-matter radius $R_{1}$ after inflation is expressed as

$$
R_{1}=r_{01} \times N_{e 1}^{\frac{1}{3}},
$$

where $r_{01}$ is the elementary particle radius of black hole elementary particles with mass $M_{1}$, $r_{01}$ is expressed as

$$
r_{01}=\frac{2 \mathrm{G} M_{1}}{\mathrm{c}^{2}} .
$$

From $\Delta r^{\prime}=\Delta r \sqrt{1-\frac{r_{s}}{\Delta r}} \fallingdotseq i \sqrt{r_{s} \times \Delta r}$ and premise 13.5, the elementary particle radius $r_{0 b}$ of the black hole elementary particle with mass $M$ at the birth of the universe is expressed as

$$
r_{0 b}=\left|\frac{\Delta r^{\prime}}{2}\right|=\frac{\sqrt{r_{s} \times \Delta r}}{2}=\frac{l_{\mathrm{p}}}{\sqrt{2}} \quad\left(s=\frac{1}{2}\right),
$$

where $l_{\mathrm{p}}$ is the Planck length.

The spacetime radius $r_{\max 1}$ before inflation is expressed as 


$$
r_{\max 1}=\left|\Delta r^{\prime}\right|=2 r_{0 b} .
$$

From equation (11.12) and $N=2$, the spacetime radius $r_{\max 2}$ for one expansion source after inflation is expressed as

$$
r_{\max 2}=\frac{3}{2} \times \frac{\mathrm{k}_{\mathrm{sp}} T_{s p}}{M}+r_{01} .
$$

The spacetime radius expansion rate $E_{R}$ in inflation is expressed as

$$
E_{R}=\frac{r_{\max 2}}{r_{\max 1}} .
$$

The maximum inter-matter radius expansion rate $E_{R m}$ in inflation is expressed as

$$
E_{R m}=\frac{R_{1}}{r_{0 b}} .
$$

The specific values are expressed as follows.

$$
\begin{gathered}
N_{e 1}=2.95 \times 10^{20} . \\
R_{1}=7.67 \times 10^{11} \text { meters } . \\
r_{01}=1.15 \times 10^{5} \text { meters } . \\
r_{0 b}=1.14 \times 10^{-35} \text { meters } . \\
r_{\text {max } 1}=2.29 \times 10^{-35} \text { meters } . \\
r_{\text {max } 2}=1.15 \times 10^{5} \text { meters } . \\
E_{R}=5.04 \times 10^{39} . \\
E_{R m}=6.71 \times 10^{46} .
\end{gathered}
$$

Therefore, in inflation, each spacetime radius expands by 39 digits or more. The maximum inter-matter radius $R_{1}$ after inflation is 6 digits or more larger than the spacetime radius $r_{\max 2}$ after inflation. 
We calculate the expansion speed and time of inflation. The change of the elementary particle radius to $\frac{\Delta r}{2}$ occurs at a speed faster than the speed of light by wave function reduction. In the case of inflation, since the elementary particle radius expands to $\frac{\Delta r}{2}$, that change is wave function enlargement. That is, inflation is a phenomenon in which each elementary particle radius expands from less than $\frac{\Delta r}{2}$ to $\frac{\Delta r}{2}$ at the same speed as wave function reduction. Therefore, when the mass $M$ is divided into each mass $M_{1}$ in inflation, the change $r_{1}^{\prime}$ in the elementary particle radius and the expansion speed $c_{1}^{\prime}$ of the elementary particle radius are expressed as follows from equation (8.75) and premise 13.7.

$$
\begin{gathered}
r_{1}^{\prime}=\mathrm{c} \times \frac{M_{t}}{M_{1}} \times \frac{s^{\prime}}{s} \times t=\mathrm{c} \times 3.87 \times 10^{20} \times t . \\
c_{1}^{\prime}=\mathrm{c} \times \frac{M_{t}}{M_{1}} \times \frac{s^{\prime}}{s}=\mathrm{c} \times 3.87 \times 10^{20} .
\end{gathered}
$$

From $\frac{\mathrm{d} r_{0}}{\mathrm{~d} t}=c_{1}^{\prime}$ and $N=1$ and $N_{e r}=N_{e 1}$, equations (13.12) and (13.16) are expressed as follows.

$$
\begin{gathered}
v_{\max } \fallingdotseq 2 N \times \frac{\mathrm{d} r_{0}}{\mathrm{~d} t}=\mathrm{c} \times 7.73 \times 10^{20} \\
v \fallingdotseq N_{e r}^{\frac{1}{3}} \times \frac{\mathrm{d} r_{0}}{\mathrm{~d} t}=\mathrm{c} \times 2.57 \times 10^{27} .
\end{gathered}
$$

From equations (13.39) and (13.40), individual radii expand at speeds more than 20 digits faster than the speed of light. The time $t_{i}$ of inflation is expressed as

$$
t_{i}=\frac{r_{01}-r_{0 b}}{c_{1}^{\prime}} \fallingdotseq \frac{r_{01}}{c_{1}^{\prime}}=9.95 \times 10^{-25} \text { seconds }
$$

From equation (13.41), the time of inflation is extremely short. Physical quantities in inflation are calculated in this way.

In summary, inflation is explained as follows. Spacetime before the birth of the universe was the imaginary spacetime by one black hole, and spacetime was repeatedly created and annihilated. In that process, the mass $M_{p}$ of the potential black hole elementary particle decreases, and the mass $M$ of the black hole elementary particle increases. When the mass $M$ of the black hole elementary particle is $M>M_{p}$, the spacetime particle is produced at $r=2 \Delta r$ and turns into the potential spacetime particle. That potential spacetime particle moves to $r=\Delta r$ from $r=2 \Delta r$ and turns into the spacetime particle at $r=\Delta r$. At this time, the black hole elementary particle collapses by the gravity of the repulsive force of itself, and individual matter is produced. The elementary particle radius of the individual matter is much larger than the elementary particle radius of the black hole elementary particle with the total mass of ordinary matter. The change of the elementary particle radius to $\frac{\Delta r}{2}$ occurs 
at a speed faster than the speed of light by wave function reduction. In the case of inflation, since the elementary particle radius expands to $\frac{\Delta r}{2}$, that change is wave function enlargement. That is, inflation is a phenomenon in which each elementary particle radius expands from less than $\frac{\Delta r}{2}$ to $\frac{\Delta r}{2}$ at the same speed as wave function reduction.

\section{Wave Function and Complex Spacetime}

In this section, we discuss the complex number of a wave function and derive the quantized coordinate transformations in complex number spacetime. From assumption 8.1, the square of the absolute value of the wave function represents the spacetime particle number density within an elementary particle. If we do not take the absolute value of the wave function, the spacetime particle number density within an elementary particle is represented by a complex number. From equation (8.29), the quantized coordinate transformations change depending on the spacetime particle number within the elementary particle. For this reason, the wave function of a complex number means that spacetime is also represented by a complex number. Therefore, the complex number of the wave function can be considered from the spacetime change.

We set the wave function as $\psi$, and we discuss the imaginary component of $\psi^{2}$. We express the wave function $\psi$ as

$$
\psi=C \times \mathrm{e}^{\mathrm{i} \theta_{w} / 2}=C\left(\cos \frac{\theta_{w}}{2}+\mathrm{i} \sin \frac{\theta_{w}}{2}\right),
$$

where $C$ is the amplitude of the wave, and $\frac{\theta_{w}}{2}$ is the phase of the wave and is expressed as

$$
\frac{\theta_{w}}{2}=\vec{k} \cdot \vec{r}-\omega t
$$

where $\vec{k}, \vec{r}$ and $\omega$ are the wave vector, position vector and angular frequency of the wave. From equation (14.1), the square $\psi^{2}$ of the wave function is expressed as

$$
\psi^{2}=C^{2} \times \mathrm{e}^{\mathrm{i} \theta_{w}}=C^{2}\left(\cos \theta_{w}+\mathrm{i} \sin \theta_{w}\right) .
$$

The following equation is obviously strange because the dimension is different, but we consider the square $\psi^{2}$ of the wave function as the following equation.

$$
\psi^{2}=C^{2}\left(\cos \theta_{w}+\mathrm{i} \sin \theta_{w}\right)=r+r^{\prime},
$$

where $r$ is the distance when spacetime change is not considered, and $r^{\prime}$ is the distance when spacetime change caused by gravity is considered. From equation (14.4), the distances $r$ and $r^{\prime}$ are expressed as follows. 


$$
\begin{gathered}
r=C^{2} \times \cos \theta_{w} . \\
r^{\prime}=C^{2} \times \operatorname{isin} \theta_{w} .
\end{gathered}
$$

From equation (14.5), equation (14.6) can be transformed as

$$
r^{\prime}=r \times \operatorname{itan} \theta_{w}
$$

If $r<r_{s}$, from equation $(9.42), r^{\prime}$ is expressed as

$$
r^{\prime}=r \sqrt{1-\frac{r_{s}}{r}}=r \times \mathrm{i} \sqrt{\frac{r_{s}-r}{r}}\left(r<r_{s}\right) .
$$

From equations (14.7) and (14.8), the following equations are shown.

$$
\begin{gathered}
\tan \theta_{w}=\sqrt{\frac{r_{s}-r}{r}} . \\
\sin \theta_{w}=\sqrt{\frac{r_{s}-r}{r_{s}}} . \\
\cos \theta_{w}=\sqrt{\frac{r}{r_{s}}} . \\
\sqrt{1-\frac{r_{s}}{r}}=\mathrm{i} \sqrt{\frac{r_{s}-r}{r}}=\operatorname{itan} \theta_{w}\left(0 \leqq r \leqq r_{s}\right) .
\end{gathered}
$$

From equation (14.4), the spacetime of an elementary particle is complex spacetime expressed as the sum of real spacetime $r$ and imaginary spacetime $r^{\prime}$. Elementary particles with $r_{0} \geqq r_{s}$ have a real spacetime, and they are elementary particles composed of spacetime particles. Elementary particles with $r_{0}<<r_{s}$ have an imaginary spacetime, and they are potential black hole elementary particles composed of potential spacetime particles. This is because after the birth of the universe, only potential black hole elementary particles have the elementary particle radius $r_{0}<<r_{s}$.

Therefore, we conclude that the imaginary component of the wave function is caused by the factor $\sqrt{1-\frac{r_{s}}{r}}$, which is the spacetime change caused by the gravity of the potential black hole elementary particle. The wave function of a complex number suggests that there are an elementary particle with real spacetime and the potential black hole elementary particle with imaginary spacetime within the elementary particle of the minimum space. For this reason, we can explain that the spacetime of an elementary particle becomes complex spacetime. Since the imaginary range is within the Schwarzschild radius, the maximum distribution 
range of the wave function is the Schwarzschild radius of the potential black hole elementary particle, not infinite.

We derive the value $\frac{c^{\prime}}{c}$ of the quantized coordinate transformations in complex spacetime. The element $n_{\max } \psi^{2}$ represents the spacetime particle number density within an elementary particle in complex spacetime. For this reason, the total spacetime particle number $n_{\max }^{\prime}$ within the elementary particle in complex spacetime is expressed as

$$
n_{\max }^{\prime}=\int_{V} n_{\max } \psi^{2} \mathrm{~d} V
$$

The interval of integration is the entire space where the wave function is distributed. From $\int_{V}|\psi|^{2} \mathrm{~d} V=\int_{V} C^{2} \mathrm{~d} V=1$ and equation (14.3), equation (14.13) is expressed as

$$
n_{\text {max }}^{\prime}=n_{\max } \mathrm{e}^{\mathrm{i} \theta_{w}} .
$$

The total spacetime particle number $n_{\max }^{\prime}$ of equation (14.14) means that spacetime particles within the elementary particle belong to both the real spacetime of the elementary particle and the imaginary spacetime of the potential black hole elementary particle.

Multiplying equation (14.14) by the kinetic energy $m_{s p} \mathrm{c}^{2}$ of one spacetime particle, it is the total kinetic energy $E_{o}^{\prime}$ of the elementary particle in complex spacetime. Therefore, from equations (14.14) and (4.35), the total kinetic energy $E_{o}^{\prime}$ is expressed as

$$
E_{o}^{\prime}=m c^{2} \times \mathrm{e}^{\mathrm{i} \theta_{w}}
$$

When only gravity is considered, the law of energy conservation is expressed by equation (9.38), so equation (14.15) is expressed as

$$
E_{o}^{\prime}=m^{\prime} c^{\prime 2}=m c^{2} \times \mathrm{e}^{\mathrm{i} \theta_{w}} .
$$

If we set the value $\frac{c^{\prime}}{c}$ as $B$, from equation (9.4), the quantized coordinate transformations are expressed as

$$
\frac{r^{\prime}}{r}=\frac{\Delta r^{\prime}}{\Delta r}=\frac{t^{\prime}}{t}=\frac{\Delta t^{\prime}}{\Delta t}=\frac{c^{\prime}}{\mathrm{c}}=\frac{m}{m^{\prime}}=B .
$$

From equation (14.17), the following equations are shown.

$$
\begin{gathered}
m=B m^{\prime} . \\
c^{\prime}=B c .
\end{gathered}
$$

From equations (14.16) and (14.18) and (14.19), the value $B$ is expressed as 


$$
B=\mathrm{e}^{\mathrm{i} \theta_{w}} .
$$

Therefore, the quantized coordinate transformations in complex spacetime are expressed as

$$
\frac{r^{\prime}}{r}=\frac{\Delta r^{\prime}}{\Delta r}=\frac{t^{\prime}}{t}=\frac{\Delta t^{\prime}}{\Delta t}=\frac{c^{\prime}}{\mathrm{c}}=\mathrm{e}^{\mathrm{i} \theta_{w}} .
$$

From quantum mechanics, equation (14.21) holds only when the elementary particle is not observed.

From the kinetic energy of an elementary particle, we derive equation (14.15). We assume that the kinetic energy $E$ in real spacetime is expressed as

$$
E=m c^{2} \times \cos \theta_{w} .
$$

When considering the spacetime change caused by gravity, kinetic energy is expressed by equation (9.50). Therefore, from equations (9.50) and (14.12) and (14.22), the kinetic energy $E^{\prime}$ in imaginary spacetime is expressed as

$$
E^{\prime}=E \sqrt{1-\frac{r_{s}}{r}}=m c^{2} \times \cos \theta_{w} \times \operatorname{itan} \theta_{w}=m c^{2} \times \operatorname{isin} \theta_{w} .
$$

From equations (14.22) and (14.23), the total kinetic energy $E_{o}^{\prime}$ of the elementary particle in complex spacetime is expressed as

$$
E_{o}^{\prime}=E+E^{\prime}=m c^{2} \times \mathrm{e}^{\mathrm{i} \theta_{w}} .
$$

Therefore, equation (14.24) is consistent with equation (14.15).

Note that the expression $r+r^{\prime}$ of equation (14.4) corresponds to $\frac{E+E^{\prime}}{m_{s p} c^{2} \times V}$.

In summary, when an elementary particle is not observed, the spacetime of the elementary particle is complex spacetime expressed as the sum of real spacetime and imaginary spacetime. Real spacetime is caused by the elementary particle composed of spacetime particles. Imaginary spacetime is caused by the potential black hole elementary particle composed of potential spacetime particles. More specifically, imaginary spacetime is caused by the factor $\sqrt{1-\frac{r_{s}}{r}}$, which is the spacetime change caused by the gravity of the potential black hole elementary particle. The quantized coordinate transformations in complex spacetime are expressed based on complex wave $\mathrm{e}^{\mathrm{i} \theta_{w}}$.

\section{How Time Advances}

In this section, we discuss how time advances. 
From the quantized coordinate transformations, we show how time advances. When considering the spacetime change caused by translational velocity and gravity, from equation (9.58), the time $t^{\prime}$ is expressed as

$$
t^{\prime}=\mathrm{t} \sqrt{1-\left(\frac{v}{\mathrm{c}}\right)^{2}} \sqrt{1-\frac{r_{s}}{r}} .
$$

First, in the case of ordinary elementary particles, from $v<\mathrm{c}$ and $r>r_{s}$, the time $t^{\prime}$ of equation (15.1) is a real number. For this reason, time advances to the future.

Second, in the case of the black hole elementary particle of $r_{0}=\frac{\Delta r}{2}<<r_{s}$, from $r<r_{s}$, the time $t^{\prime}$ of equation (15.1) is an imaginary number. For this reason, from section 12, time advances to the past. When time returns to the past and spacetime is annihilated, the factor $\sqrt{1-\frac{r_{s}}{r}}$ of spacetime change caused by gravity disappears, so the time $t^{\prime}$ of equation (15.1) is a real number and time advances to the future. Therefore, in this case, spacetime is repeatedly created and annihilated.

Third, in the case of charged particles without spacetime particles, their kinetic energy is zero, so they cannot be observed. When a particle is not observed, its spacetime is complex spacetime expressed as the sum of the real spacetime by spacetime particles and the imaginary spacetime by potential spacetime particles. If there are no spacetime particles, spacetime is the imaginary spacetime by potential spacetime particles, and time advances to the past. When spacetime is annihilated, the time $t^{\prime}$ is a real number, and time advances to the future. Therefore, in this case, spacetime is repeatedly created and annihilated. This is the spacetime in the time when matter and antimatter undergo pair production and annihilation within the quantized minimum time $\Delta t$.

In this way, if spacetime is a real spacetime, time advances to the future, and if spacetime is an imaginary spacetime, time advances to the past. However, when time advances to the past and spacetime is annihilated, time advances to the future. Therefore, in imaginary spacetime, spacetime is repeatedly created and annihilated.

We discuss how time advances in the complex spacetime of a wave function. The imaginary component of the wave function is represented as an imaginary number by the spacetime change caused by gravity. For this reason, in the imaginary component of the wave function, time advances to the past. Therefore, the time of the wave function is a complex time represented as the sum of the real time in which time advances to the future and the imaginary time in which time advances to the past. That is, the time of the wave function advances to the past at the same time as it advances to the future.

We discuss the time of elementary particles when they are not observed. The time of elementary particles at the time of observation is expressed by equation (15.1), and time advances as described above. However, from equation (14.21), the time $t^{\prime}$ of elementary particles when they are not observed is expressed as 


$$
t^{\prime}=t \mathrm{e}^{\mathrm{i} \theta_{w}}=N \Delta t \times \mathrm{e}^{\mathrm{i} \theta_{w}} .
$$

Therefore, when elementary particles are not observed, their time is a complex time represented as the sum of the real time in which time advances to the future and the imaginary time in which time advances to the past. When the complex time is observed, from the absolute value of equation (15.2), time is expressed as

$$
\left|t^{\prime}\right|=t=N \Delta t
$$

Therefore, when elementary particles are observed, time is real time and advances to the future. However, the actual observable time is only the time for each $\Delta t$. The time $((N-$ 1) $\Delta t<t<N \Delta t$ ) within the quantized minimum time $\Delta t$ cannot be observed. For this reason, the time within the quantized minimum time $\Delta t$ is the complex time. From this, we observe that the time when observed for each $\Delta t$ advances only to the future, but the time within the quantized minimum time $\Delta t$ advances to the future and at the same time to the past.

In summary, if spacetime is a real spacetime, time advances to the future, and if spacetime is an imaginary spacetime, time advances to the past. However, when time advances to the past and spacetime is annihilated, time advances to the future. Therefore, in imaginary spacetime, spacetime is repeatedly created and annihilated. The time in complex spacetime advances to the past at the same time as it advances to the future. The time when not observed is a complex time, time advances to the past at the same time as it advances to the future.

\section{Lorentz Transformations in Graviton Coordinates}

In this section, we derive the Lorentz transformations in graviton coordinates and show the invariance of Maxwell's equations under the Lorentz transformations in graviton coordinates. The quantized coordinate transformations are valid only in graviton coordinates. All spacetime changes in this paper are based on quantized coordinate transformations. Therefore, we derive the Lorentz transformations from the quantized coordinate transformations.

In this section, the $t$ frame is the rest frame, the $t^{\prime}$ frame is the frame moving at a translational velocity $v$ against the $t$ frame.

\subsection{Speed of Light of a Complex Number}

We show the speed of light of a complex number that satisfies the law of energy conservation. The Lorentz transformations are derived from the speed of light of a complex number. 
The law of energy conservation in spacetime change caused by translational velocity is expressed by equation (9.19). For this reason, the velocity relation of $c^{2}=c^{2}+v^{2}$ must hold. From equation (8.38), the rotational velocity and the velocity of radius are equivalent. Therefore, regardless of the direction of the translational velocity $v$, the direction of the translational velocity $v$ must be perpendicular to the direction of the velocity $c^{\prime}$ of the radius. To satisfy this velocity relation, we consider the following light speed $c^{\prime}$ of a complex number.

$$
c^{\prime}=\mathrm{c} \times \mathrm{e}^{\mathrm{i} \theta_{v}}=\mathrm{c} \sqrt{1-\left(\frac{v}{\mathrm{c}}\right)^{2}}+\mathrm{i} v
$$

where $\mathrm{e}^{\mathrm{i} \theta_{v}}$ is expressed as

$$
\mathrm{e}^{\mathrm{i} \theta_{v}}=\cos \theta_{v}+\operatorname{isin} \theta_{v}=\sqrt{1-\left(\frac{v}{\mathrm{c}}\right)^{2}}+\mathrm{i} \frac{v}{\mathrm{c}},
$$

where $\cos \theta_{v}$ and $\sin \theta_{v}$ are expressed as follows.

$$
\begin{gathered}
\cos \theta_{v}=\sqrt{1-\left(\frac{v}{\mathrm{c}}\right)^{2}} . \\
\sin \theta_{v}=\frac{v}{\mathrm{c}} . \\
\tan \theta_{v}=\frac{v}{\mathrm{c} \sqrt{1-\left(\frac{v}{\mathrm{c}}\right)^{2}}} .
\end{gathered}
$$

The square of the absolute value of equation (16.1) is expressed as

$$
\left|c^{\prime}\right|^{2}=c^{2}=R_{e}\left(c^{\prime}\right)^{2}+I_{m}\left(c^{\prime}\right)^{2},
$$

where $R_{e}\left(c^{\prime}\right)$ is the rotational velocity ( = the velocity of the radius) of spacetime particles of the $t^{\prime}$ frame observed from the $t$ frame, $I_{m}\left(c^{\prime}\right)$ is the translational velocity of spacetime particles of the $t^{\prime}$ frame observed from the $t$ frame, and $R_{e}\left(c^{\prime}\right)$ and $I_{m}\left(c^{\prime}\right)$ are expressed as follows.

$$
\begin{gathered}
R_{e}\left(c^{\prime}\right)=c \sqrt{1-\left(\frac{v}{c}\right)^{2}} . \\
I_{m}\left(c^{\prime}\right)=v .
\end{gathered}
$$

Therefore, the speed of light of a complex number in equation (16.1) satisfies the velocity relation of equation (9.20). The speed $c^{\prime}$ of light of a complex number represents the velocity of a directional relationship that is mathematically always perpendicular, and the actual velocity is not a complex number. For this reason, the actual velocity must be considered by equations (16.7) and (16.8). 


\subsection{Derivation of the Lorentz Transformations in Graviton Coordinates}

We derive the Lorentz transformations in graviton coordinates and discuss their physical meaning.

The normal Lorentz transformations are expressed as follows.

$$
\begin{gathered}
x^{\prime}=\frac{x-v t}{\sqrt{1-\left(\frac{v}{\mathrm{c}}\right)^{2}}} . \\
y^{\prime}=y . \\
z^{\prime}=z . \\
t^{\prime}=\frac{t-v x / \mathrm{c}^{2}}{\sqrt{1-\left(\frac{v}{\mathrm{c}}\right)^{2}}} . \\
x=\frac{x^{\prime}+v t^{\prime}}{\sqrt{1-\left(\frac{v}{\mathrm{c}}\right)^{2}}} . \\
y=y^{\prime} . \\
z=z^{\prime} . \\
t=\frac{t^{\prime}+v x^{\prime} / \mathrm{c}^{2}}{\sqrt{1-\left(\frac{v}{\mathrm{c}}\right)^{2}}} .
\end{gathered}
$$

Since graviton coordinates have only $r$ and $t$, the normal Lorentz transformations in graviton coordinates are expressed as follows.

$$
\begin{aligned}
r^{\prime} & =\frac{r-v t}{\sqrt{1-\left(\frac{v}{\mathrm{c}}\right)^{2}}} . \\
t^{\prime} & =\frac{t-v r / \mathrm{c}^{2}}{\sqrt{1-\left(\frac{v}{\mathrm{c}}\right)^{2}}} . \\
r & =\frac{r^{\prime}+v t^{\prime}}{\sqrt{1-\left(\frac{v}{\mathrm{c}}\right)^{2}}} . \\
t & =\frac{t^{\prime}+v r^{\prime} / \mathrm{c}^{2}}{\sqrt{1-\left(\frac{v}{\mathrm{c}}\right)^{2}}} .
\end{aligned}
$$


We show the Lorentz transformations in graviton coordinates. From equation (16.1), the quantized coordinate transformations based on the speed of light of a complex number are expressed as

$$
\frac{r^{\prime}}{r}=\frac{\Delta r^{\prime}}{\Delta r}=\frac{t^{\prime}}{t}=\frac{\Delta t^{\prime}}{\Delta t}=\frac{c^{\prime}}{\mathrm{c}}=\mathrm{e}^{\mathrm{i} \theta_{v}}
$$

From equation (16.21), the radius $r^{\prime}$ and the time $t^{\prime}$ are expressed as follows.

$$
\begin{aligned}
& r^{\prime}=r \times \mathrm{e}^{\mathrm{i} \theta_{v}}=r\left(\sqrt{1-\left(\frac{v}{\mathrm{c}}\right)^{2}}+\mathrm{i} \frac{v}{\mathrm{c}}\right) . \\
& t^{\prime}=t \times \mathrm{e}^{\mathrm{i} \theta_{v}}=t\left(\sqrt{1-\left(\frac{v}{\mathrm{c}}\right)^{2}}+\mathrm{i} \frac{v}{\mathrm{c}}\right) .
\end{aligned}
$$

From $r=\mathrm{c} t$ and $t=\frac{r}{\mathrm{c}}$, equations (16.22) and (16.23) are expressed as follows.

$$
\begin{aligned}
& r^{\prime}=r \times \mathrm{e}^{\mathrm{i} \theta}=r \sqrt{1-\left(\frac{v}{\mathrm{c}}\right)^{2}}+\mathrm{i} v t . \\
& t^{\prime}=t \times \mathrm{e}^{\mathrm{i} \theta_{v}}=t \sqrt{1-\left(\frac{v}{\mathrm{c}}\right)^{2}}+\mathrm{i} \frac{v r}{\mathrm{c}^{2}} .
\end{aligned}
$$

When we rearrange equations (16.17) and (16.18), they are expressed as follows.

$$
\begin{aligned}
& r=r^{\prime} \sqrt{1-\left(\frac{v}{c}\right)^{2}}+v t . \\
& t=t^{\prime} \sqrt{1-\left(\frac{v}{c}\right)^{2}}+\frac{v r}{c^{2}} .
\end{aligned}
$$

Equation (16.24) is almost the same as equation (16.26), and equation (16.25) is almost the same as equation (16.27). Therefore, we conclude that equations (16.22) and (16.23) represent the Lorentz transformations in graviton coordinates.

Their inverse transformations are expressed as follows.

$$
\begin{gathered}
\mathrm{e}^{-\mathrm{i} \theta_{v}}=\cos \theta_{v}-\mathrm{i} \sin \theta_{v}=\sqrt{1-\left(\frac{v}{\mathrm{c}}\right)^{2}}-\mathrm{i} \frac{v}{\mathrm{c}} \\
r=r^{\prime} \times \mathrm{e}^{-\mathrm{i} \theta_{v}}=r^{\prime}\left(\sqrt{1-\left(\frac{v}{\mathrm{c}}\right)^{2}}-\mathrm{i} \frac{v}{\mathrm{c}}\right) . \\
t=t^{\prime} \times \mathrm{e}^{-\mathrm{i} \theta_{v}}=t^{\prime}\left(\sqrt{1-\left(\frac{v}{\mathrm{c}}\right)^{2}}-\mathrm{i} \frac{v}{\mathrm{c}}\right) .
\end{gathered}
$$




$$
c^{* *}=\mathrm{c} \times \mathrm{e}^{-\mathrm{i} \theta_{v}}=\mathrm{c} \sqrt{1-\left(\frac{v}{\mathrm{c}}\right)^{2}}-\mathrm{i} v
$$

where $c^{*}$ is the complex conjugate of $c^{\prime}$.

We show the Lorentz transformations in graviton coordinates as Cartesian coordinates form. Since the velocity in graviton coordinates is the same velocity in all directions, the following equations hold.

$$
\begin{gathered}
r=x=y=z=\mathrm{c} t \\
r^{\prime}=x^{\prime}=y^{\prime}=z^{\prime}=\mathrm{c} t^{\prime} . \\
v=v_{x}=v_{y}=v_{z},
\end{gathered}
$$

where $v_{x}$ is the velocity in the x-direction, $v_{y}$ is the velocity in the y-direction, and $v_{z}$ is the velocity in the z-direction. Therefore, the Lorentz transformations in graviton coordinates in Cartesian coordinates form are expressed as follows.

$$
\begin{aligned}
& x^{\prime}=x \times \mathrm{e}^{\mathrm{i} \theta_{v}}=x\left(\sqrt{1-\left(\frac{v}{\mathrm{c}}\right)^{2}}+\mathrm{i} \frac{v}{\mathrm{c}}\right) . \\
& y^{\prime}=y \times \mathrm{e}^{\mathrm{i} \theta_{v}}=y\left(\sqrt{1-\left(\frac{v}{\mathrm{c}}\right)^{2}}+\mathrm{i} \frac{v}{\mathrm{c}}\right) . \\
& z^{\prime}=z \times \mathrm{e}^{\mathrm{i} \theta_{v}}=z\left(\sqrt{1-\left(\frac{v}{\mathrm{c}}\right)^{2}}+\mathrm{i} \frac{v}{\mathrm{c}}\right) . \\
& t^{\prime}=t \times \mathrm{e}^{\mathrm{i} \theta_{v}}=t\left(\sqrt{1-\left(\frac{v}{\mathrm{c}}\right)^{2}}+\mathrm{i} \frac{v}{\mathrm{c}}\right) .
\end{aligned}
$$

We discuss the physical meaning of the Lorentz transformations in graviton coordinates.

Their real part is the time, distance and velocity for the motion of radius or the rotational motion or the motion within the $t^{\prime}$ frame.

Their imaginary part is the time, distance and velocity for the translational motion or the motion of the entire $t^{\prime}$ frame.

For example, when $v \fallingdotseq c$, the Lorentz transformations in graviton coordinates are expressed as follows.

$$
c^{\prime}=\mathrm{c} \times \mathrm{e}^{\mathrm{i} \theta_{v}} \fallingdotseq \mathrm{ic}
$$




$$
\begin{gathered}
r^{\prime}=r \times \mathrm{e}^{\mathrm{i} \theta_{v}} \fallingdotseq \mathrm{ict}=\mathrm{i} r \\
t^{\prime}=t \times \mathrm{e}^{\mathrm{i} \theta_{v}} \fallingdotseq \mathrm{i} t
\end{gathered}
$$

Therefore, when $v \fallingdotseq \mathrm{c}$, there is no time, distance and velocity for the motion within the $t^{\prime}$ frame, but only the time, distance and velocity for the motion of the entire $t^{\prime}$ frame. That is, the motion within the $t^{\prime}$ frame stops, but the motion of the entire $t^{\prime}$ frame moves at almost the speed of light, and the time $I_{m}\left(t^{\prime}\right)$ of the entire $t^{\prime}$ frame has elapsed. In this way, the element of the motion of radius and the element of translational motion can be considered separately. Equations (16.38) to (16.40) mean these.

\subsection{Law of the Speed Constancy}

We show the law of speed constancy. This law is used to show the invariance of Maxwell's equations under the Lorentz transformations in graviton coordinates. The variable transformations in differentiation based on equation (16.21) are expressed as follows.

$$
\begin{aligned}
& \mathrm{d} r^{\prime}=\mathrm{d} r \times \mathrm{e}^{\mathrm{i} \theta_{v}} . \\
& \mathrm{d} x^{\prime}=\mathrm{d} x \times \mathrm{e}^{\mathrm{i} \theta_{v}} . \\
& \mathrm{d} y^{\prime}=\mathrm{d} y \times \mathrm{e}^{\mathrm{i} \theta_{v}} . \\
& \mathrm{d} z^{\prime}=\mathrm{d} z \times \mathrm{e}^{\mathrm{i} \theta_{v}} . \\
& \mathrm{d} t^{\prime}=\mathrm{d} t \times \mathrm{e}^{\mathrm{i} \theta_{v}} .
\end{aligned}
$$

Based on equations (16.41) to (16.45), differentiating the spatial coordinates of the $t^{\prime}$ frame with time $t^{\prime}$, they are expressed as follows.

$$
\begin{aligned}
& \frac{\mathrm{d} r^{\prime}}{\mathrm{d} t^{\prime}}=\frac{\mathrm{d} r}{\mathrm{~d} t} . \\
& \frac{\mathrm{d} x^{\prime}}{\mathrm{d} t^{\prime}}=\frac{\mathrm{d} x}{\mathrm{~d} t} . \\
& \frac{\mathrm{d} y^{\prime}}{\mathrm{d} t^{\prime}}=\frac{\mathrm{d} y}{\mathrm{~d} t} .
\end{aligned}
$$




$$
\frac{\mathrm{d} z^{\prime}}{\mathrm{d} t^{\prime}}=\frac{\mathrm{d} z}{\mathrm{~d} t}
$$

Therefore, the velocity in the $t^{\prime}$ frame is consistent with the velocity in the $t$ frame. That is, the velocity in the $t^{\prime}$ frame observed from the $t^{\prime}$ frame is equal to the velocity in the $t$ frame observed from the $t$ frame. For example, the speed of light is always observed as the same speed if it belongs to the same reference frame as the observer. Since these are not limited to the speed of light, these are the law of speed constancy.

\subsection{Invariance of Maxwell's Equations Under the Lorentz Transformations in Graviton Coordinates}

We show the invariance of Maxwell's equations under the Lorentz transformations in graviton coordinates.

Maxwell's equations are shown as follows.

$$
\begin{gathered}
\nabla \times \vec{E}=-\frac{\partial \vec{B}}{\partial t}, \\
\nabla \times \vec{H}=\frac{\partial \vec{D}}{\partial t}+\vec{j}, \\
\nabla \cdot \vec{D}=\rho, \\
\nabla \cdot \vec{B}=0,
\end{gathered}
$$

where $\vec{E}$ is the electric field, $\vec{B}$ is the magnetic field, $\vec{H}$ is the magnetic field strength, $\vec{D}$ is the electric displacement, $\vec{j}$ is the electric current density, and $\rho$ is the electric charge density.

We transform the physical quantities of the $t^{\prime}$ frame based on the Lorentz transformations in graviton coordinates. In the following, the elements $t, \vec{E}, \vec{D}, \vec{H}, \vec{B}, \vec{j}, \rho$ and $\nabla$ are the elements of the $t$ frame, and the elements $t^{\prime}, \overrightarrow{E^{\prime}}, \overrightarrow{D^{\prime}}, \overrightarrow{H^{\prime}}, \overrightarrow{B^{\prime}}, \overrightarrow{j^{\prime}}, \rho^{\prime}$ and $\nabla^{\prime}$ are the elements of the $t^{\prime}$ frame. As mentioned above, the $t$ frame is the rest frame, the $t^{\prime}$ frame is the frame moving at a translational velocity $v$ against the $t$ frame.

First, from equations (16.42) to (16.45), $\frac{\partial}{\partial t^{\prime}}$ and $\nabla^{\prime}$ are transformed as follows.

$$
\begin{gathered}
\frac{\partial}{\partial t^{\prime}}=\frac{1}{\mathrm{e}^{\mathrm{i} \theta_{v}}} \times \frac{\partial}{\partial t} . \\
\nabla^{\prime}=\left(\frac{\partial}{\partial x^{\prime}}, \frac{\partial}{\partial y^{\prime}}, \frac{\partial}{\partial z^{\prime}}\right)=\frac{1}{\mathrm{e}^{\mathrm{i} \theta_{v}}} \times \nabla .
\end{gathered}
$$


Second, since $\overrightarrow{E^{\prime}}, \overrightarrow{D^{\prime}}, \overrightarrow{H^{\prime}}$ and $\overrightarrow{B^{\prime}}$ are all inversely proportional to the square of the radius $r^{\prime}$, they are transformed as follows.

$$
\begin{aligned}
& \overrightarrow{E^{\prime}}=\frac{1}{\mathrm{e}^{\mathrm{i} 2 \theta_{v}}} \times \vec{E} . \\
& \overrightarrow{D^{\prime}}=\frac{1}{\mathrm{e}^{\mathrm{i} 2 \theta_{v}}} \times \vec{D} . \\
& \vec{H}^{\prime}=\frac{1}{\mathrm{e}^{\mathrm{i} 2 \theta_{v}}} \times \vec{H} . \\
& \overrightarrow{B^{\prime}}=\frac{1}{\mathrm{e}^{\mathrm{i} 2 \theta_{v}}} \times \vec{B} .
\end{aligned}
$$

Third, we discuss the electric charge density $\rho^{\prime}$. The electric charge densities $\rho^{\prime}$ and $\rho$ are expressed as follows.

$$
\begin{gathered}
\rho=\frac{\mathrm{d} Q}{\mathrm{~d} V}, \\
\rho^{\prime}=\frac{\mathrm{d} Q^{\prime}}{\mathrm{d} V^{\prime}},
\end{gathered}
$$

where $Q$ is the amount of electric charge of the $t$ frame, $V$ is the volume of $Q$ of the $t$ frame, $Q^{\prime}$ is the amount of electric charge of the $t^{\prime}$ frame, $V^{\prime}$ is the volume of $Q^{\prime}$ of the $t^{\prime}$ frame. The amount of electric charge does not change in either the $t$ frame or the $t^{\prime}$ frame, so the following equation holds.

$$
\mathrm{d} Q=\mathrm{d} Q^{\prime}
$$

The volume element $\mathrm{d} V^{\prime}$ is expressed as

$$
\mathrm{d} V^{\prime}=\Delta V^{\prime}=4 \pi r^{\prime 2} \times \Delta r^{\prime}=\mathrm{d} V \times \mathrm{e}^{\mathrm{i} 3 \theta} .
$$

Therefore, the electric charge density $\rho^{\prime}$ is transformed as

$$
\rho^{\prime}=\frac{\mathrm{d} Q^{\prime}}{\mathrm{d} V^{\prime}}=\frac{1}{\mathrm{e}^{\mathrm{i} 3 \theta_{v}}} \times \frac{\mathrm{d} Q}{\mathrm{~d} V}=\frac{1}{\mathrm{e}^{\mathrm{i} 3 \theta_{v}}} \times \rho .
$$

Fourth, we discuss the electric current density $\overrightarrow{j^{\prime}}$. The electric current densities $\vec{j}$ and $\overrightarrow{j^{\prime}}$ are expressed as follows.

$$
\vec{j}=\rho \overrightarrow{v_{0}}
$$




$$
\overrightarrow{j^{\prime}}=\rho^{\prime} \overrightarrow{v_{0}^{\prime}}
$$

where $\overrightarrow{v_{0}}$ is the velocity vector of the electric charge density $\rho$, and $\overrightarrow{v_{0}^{\prime}}$ is the velocity vector of the electric charge density $\rho^{\prime}$. The velocities $\overrightarrow{v_{0}}$ and $\overrightarrow{v_{0}^{\prime}}$ are expressed as follows.

$$
\begin{aligned}
& \overrightarrow{v_{0}^{\prime}}=\left(\frac{\mathrm{d} x^{\prime}}{\mathrm{d} t^{\prime}}, \frac{\mathrm{d} y^{\prime}}{\mathrm{d} t^{\prime}}, \frac{\mathrm{d} z^{\prime}}{\mathrm{d} t^{\prime}}\right) . \\
& \overrightarrow{v_{0}}=\left(\frac{\mathrm{d} x}{\mathrm{~d} t}, \frac{\mathrm{d} y}{\mathrm{~d} t}, \frac{\mathrm{d} z}{\mathrm{~d} t}\right) .
\end{aligned}
$$

From equations (16.47) to (16.49), the following equation holds between $\overrightarrow{v_{0}}$ and $\overrightarrow{v_{0}^{\prime}}$.

$$
\overrightarrow{v_{0}^{\prime}}=\overrightarrow{v_{0}} \text {. }
$$

From equations (16.64) and (16.69), the electric current density $\overrightarrow{j^{\prime}}$ is expressed as

$$
\overrightarrow{j^{\prime}}=\rho^{\prime} \overrightarrow{v_{0}^{\prime}}=\frac{1}{\mathrm{e}^{\mathrm{i} 3 \theta_{v}}} \times \rho \overrightarrow{v_{0}}=\frac{1}{\mathrm{e}^{\mathrm{i} 3 \theta_{v}}} \times \vec{j} .
$$

In summary, based on the Lorentz transformations in graviton coordinates, the physical quantities of the $t^{\prime}$ frame are transformed as follows.

$$
\begin{aligned}
\frac{\partial}{\partial t^{\prime}} & =\frac{1}{\mathrm{e}^{\mathrm{i} \theta_{v}}} \times \frac{\partial}{\partial t} . \\
\nabla^{\prime} & =\frac{1}{\mathrm{e}^{\mathrm{i} \theta_{v}}} \times \nabla . \\
\vec{E}^{\prime} & =\frac{1}{\mathrm{e}^{\mathrm{i} 2 \theta_{v}}} \times \vec{E} . \\
\overrightarrow{D^{\prime}} & =\frac{1}{\mathrm{e}^{\mathrm{i} 2 \theta_{v}}} \times \vec{D} . \\
\vec{H}^{\prime} & =\frac{1}{\mathrm{e}^{\mathrm{i} 2 \theta_{v}}} \times \vec{H} . \\
\vec{B}^{\prime} & =\frac{1}{\mathrm{e}^{\mathrm{i} 2 \theta_{v}}} \times \vec{B} . \\
\rho^{\prime} & =\frac{1}{\mathrm{e}^{\mathrm{i} 3 \theta_{v}}} \times \rho .
\end{aligned}
$$




$$
\overrightarrow{j^{\prime}}=\frac{1}{\mathrm{e}^{\mathrm{i} 3 \theta_{v}}} \times \vec{j} .
$$

From these, we show the invariance of Maxwell's equations under the Lorentz transformations in graviton coordinates. Maxwell's equations of the $t^{\prime}$ frame are shown as follows.

$$
\begin{gathered}
\nabla^{\prime} \times \overrightarrow{E^{\prime}}=-\frac{\partial \overrightarrow{B^{\prime}}}{\partial t^{\prime}}, \\
\nabla^{\prime} \times \overrightarrow{H^{\prime}}=\frac{\partial \overrightarrow{D^{\prime}}}{\partial t^{\prime}}+\overrightarrow{j^{\prime}}, \\
\nabla^{\prime} \cdot \overrightarrow{D^{\prime}}=\rho^{\prime}, \\
\nabla^{\prime} \cdot \overrightarrow{B^{\prime}}=0,
\end{gathered}
$$

Transforming the individual elements in these equations, they are expressed as follows.

$$
\begin{gathered}
\frac{1}{\mathrm{e}^{\mathrm{i} 3 \theta_{v}}} \times(\nabla \times \vec{E})=\frac{1}{\mathrm{e}^{\mathrm{i} 3 \theta_{v}}} \times\left(-\frac{\partial \vec{B}}{\partial t}\right), \\
\frac{1}{\mathrm{e}^{\mathrm{i} 3 \theta_{v}}} \times(\nabla \times \vec{H})=\frac{1}{\mathrm{e}^{\mathrm{i} 3 \theta_{v}}} \times\left(\frac{\partial \vec{D}}{\partial t}+\vec{j}\right), \\
\frac{1}{\mathrm{e}^{\mathrm{i} 3 \theta_{v}}} \times(\nabla \cdot \vec{D})=\frac{1}{\mathrm{e}^{\mathrm{i} 3 \theta_{v}}} \times(\rho), \\
\frac{1}{\mathrm{e}^{\mathrm{i} 3 \theta_{v}}} \times(\nabla \cdot \vec{B})=0,
\end{gathered}
$$

Therefore, they are transformed to Maxwell's equations of the $t$ frame, and Maxwell's equations are invariant under the Lorentz transformations in graviton coordinates. Note that Maxwell's equations are invariant under arbitrary quantized coordinate transformations. This means that if electromagnetic phenomenon belongs to the same frame of reference as an observer, it can be described as the same form. That is, under the quantized coordinate transformations, the relativity principle for Maxwell's equations holds. 


\section{Physical Quantities of the Frame Moving at Velocity $v$}

In this section, we discuss the physical quantities of the $t^{\prime}$ frame observed from the $t$ frame, where the $t$ frame is the rest frame, and the $t^{\prime}$ frame is the frame moving at a translational velocity $v$ against the $t$ frame. When the physical quantity of the $t^{\prime}$ frame is calculated by the time and distance of the $t$ frame, it becomes the physical quantity of the $t^{\prime}$ frame observed from the $t$ frame.

\subsection{The Velocity of the $t^{\prime}$ Frame Observed From the $t$ Frame}

We show the velocity of the $t^{\prime}$ frame observed from the $t$ frame. From equation (16.41) and $\frac{\mathrm{d} r}{\mathrm{~d} t}=\mathrm{c}$, when the distance $r^{\prime}$ is differentiated by time $t$, its velocity is expressed as

$$
\frac{\mathrm{d} r^{\prime}}{\mathrm{d} t}=\mathrm{c} \times \mathrm{e}^{\mathrm{i} \theta_{v}}=\mathrm{c} \sqrt{1-\left(\frac{v}{\mathrm{c}}\right)^{2}}+\mathrm{i} v .
$$

From equation (16.46) and $\frac{\mathrm{d} r}{\mathrm{~d} t}=\mathrm{c}$, when the distance $r^{\prime}$ is differentiated by the time $t^{\prime}$, its velocity is expressed as

$$
\frac{\mathrm{d} r^{\prime}}{\mathrm{d} t^{\prime}}=\frac{\mathrm{d} r}{\mathrm{~d} t}=\mathrm{c}
$$

Equation (17.1) is the speed of light of the $t^{\prime}$ frame observed from the $t$ frame. Equation (17.2) is the speed of light of the $t^{\prime}$ frame observed from the $t^{\prime}$ frame. Therefore, the physical meaning differs between the case of differentiating with $t$ and the case of differentiating with $t^{\prime}$, so the speed is different.

\subsection{Electromagnetic Waves of the $t^{\prime}$ Frame Observed From the $t$ Frame}

From the Lorentz transformations in graviton coordinates and Maxwell's equations, we derive electromagnetic waves of the $t^{\prime}$ frame observed from the $t$ frame.

The wave equations of electric and magnetic fields are expressed as follows.

$$
\begin{aligned}
& \nabla^{2} \vec{E}=\frac{1}{c^{2}} \frac{\partial^{2} \vec{E}}{\partial t^{2}} . \\
& \nabla^{2} \vec{H}=\frac{1}{c^{2}} \frac{\partial^{2} \vec{H}}{\partial t^{2}} .
\end{aligned}
$$


When the physical quantity of the $t^{\prime}$ frame is calculated by the time and distance of the $t$ frame, it becomes the physical quantity of the $t^{\prime}$ frame observed from the $t$ frame. Therefore, the wave equations for the electromagnetic waves of the $t^{\prime}$ frame observed from the $t$ frame are expressed as follows.

$$
\begin{aligned}
\nabla^{2} \vec{E}^{\prime} & =\frac{1}{\left(\mathrm{c} \times \mathrm{e}^{\mathrm{i} \theta_{v}}\right)^{2}} \frac{\partial^{2} \vec{E}}{\partial t^{2}} . \\
\nabla^{2} \vec{H}^{\prime} & =\frac{1}{\left(\mathrm{c} \times \mathrm{e}^{\mathrm{i} \theta_{v}}\right)^{2}} \frac{\partial^{2} \vec{H}}{\partial t^{2}} .
\end{aligned}
$$

The electromagnetic waves of the $t^{\prime}$ frame have only the rotational kinetic energy $E_{r}^{\prime}$. For this reason, from equation (10.16), their angular frequency $\omega^{\prime}$ is expressed as

$$
\omega^{\prime}=2 \pi f^{\prime}=2 \pi f \sqrt{1-\left(\frac{v}{c}\right)^{2}}=\omega \sqrt{1-\left(\frac{v}{c}\right)^{2}},
$$

where $\omega^{\prime}$ is the angular frequency of the electromagnetic waves of the $t^{\prime}$ frame observed from the $t$ frame, $\omega$ is the angular frequency of the electromagnetic waves of the $t^{\prime}$ frame observed from the $t^{\prime}$ frame.

Equations (17.5) and (17.6) cannot be solved because their variables ( $\vec{E}$ and $\vec{E}^{\prime}, \vec{H}$ and $\left.\vec{H}^{\prime}\right)$ are different. For this reason, we assume the following.

$$
\begin{aligned}
& \overrightarrow{E^{\prime}}=\vec{E}=\vec{E}_{0}(x, y, z) \times \mathrm{e}^{\mathrm{i} \omega^{\prime} t} . \\
& \overrightarrow{H^{\prime}}=\vec{H}=\vec{H}_{0}(x, y, z) \times \mathrm{e}^{\mathrm{i} \omega^{\prime} t} .
\end{aligned}
$$

From equations (17.8) and (17.9), equations (17.5) and (17.6) are expressed as follows.

$$
\begin{aligned}
& \nabla^{2} \vec{E}_{0}+k^{2} \vec{E}_{0}=0 \\
& \nabla^{2} \vec{H}_{0}+k^{\prime 2} \vec{H}_{0}=0
\end{aligned}
$$

where $k^{\prime}$ is the wavenumber of the $t^{\prime}$ frame observed from the $t$ frame and is expressed as

$$
k^{\prime}=\frac{\omega^{\prime}}{\mathrm{c} \times \mathrm{e}^{\mathrm{i} \theta_{v}}} .
$$

Equations (17.10) and (17.11) can be solved. For example, plane waves propagating in the z-direction are expressed as follows.

$$
E_{x}^{\prime}=A_{1} \mathrm{e}^{\mathrm{i}\left(k^{\prime} z+\omega^{\prime} t\right)}+A_{2} \mathrm{e}^{\mathrm{i}\left(-k^{\prime} z+\omega^{\prime} t\right)}
$$




$$
H_{y}^{\prime}=A_{3} \mathrm{e}^{\mathrm{i}\left(k^{\prime} z+\omega^{\prime} t\right)}+A_{4} \mathrm{e}^{\mathrm{i}\left(-k^{\prime} z+\omega^{\prime} t\right)},
$$

where $A_{1}, A_{2}, A_{3}$ and $A_{4}$ are the amplitude of the waves of arbitrary constant, $E_{x}^{\prime}$ is the x-component of the electric field, and $H_{y}^{\prime}$ is the y-component of the magnetic field. The phase velocity $c^{\prime}$ of the electromagnetic waves is expressed as

$$
c^{\prime}=\frac{\mathrm{d} z}{\mathrm{~d} t}=\frac{\omega^{\prime}}{k^{\prime}}=\mathrm{c} \times \mathrm{e}^{\mathrm{i} \theta_{v}}=\mathrm{c} \sqrt{1-\left(\frac{v}{\mathrm{c}}\right)^{2}}+\mathrm{i} v .
$$

Therefore, the speed of electromagnetic waves of the $t^{\prime}$ frame observed from the $t$ frame is consistent with equation (17.1). Equation (17.15) means that electromagnetic waves have the velocity $\mathrm{c} \sqrt{1-\left(\frac{v}{c}\right)^{2}}$ of radius and the translational velocity $v$. More precisely, the translational velocity $v$ is the velocity of the medium of electromagnetic waves.

\subsection{Maxwell's Equations of the $t^{\prime}$ Frame Observed From the $t$ Frame}

We show Maxwell's equations of the $t^{\prime}$ frame observed from the $t$ frame. When the physical quantity of the $t^{\prime}$ frame is calculated by the time and distance of the $t$ frame, it becomes the physical quantity of the $t^{\prime}$ frame observed from the $t$ frame. For this reason, Maxwell's equations of the $t^{\prime}$ frame observed from the $t$ frame are expressed as follows.

$$
\begin{gathered}
\nabla \times{\overrightarrow{E^{\prime}}}^{\prime}=\frac{1}{\mathrm{e}^{\mathrm{i} 2 \theta_{v}}} \times\left(-\frac{\partial \vec{B}}{\partial t}\right), \\
\nabla \times \overrightarrow{H^{\prime}}=\frac{1}{\mathrm{e}^{\mathrm{i} 2 \theta_{v}}} \times\left(\frac{\partial \vec{D}}{\partial t}+\vec{j}\right), \\
\nabla \cdot \overrightarrow{D^{\prime}}=\frac{1}{\mathrm{e}^{\mathrm{i} 2 \theta_{v}}} \times(\rho), \\
\nabla \cdot \overrightarrow{B^{\prime}}=0,
\end{gathered}
$$

From equation (17.17), the electric current density $\overrightarrow{j^{\prime \prime}}$ of the $t^{\prime}$ frame observed from the $t$ frame is expressed as

$$
\overrightarrow{j^{\prime \prime}}=\frac{1}{\mathrm{e}^{\mathrm{i} 2 \theta_{v}}} \times \vec{j} .
$$

From equation (17.18), the electric charge density $\rho^{\prime \prime}$ of the $t^{\prime}$ frame observed from the $t$ frame is expressed as 


$$
\rho^{\prime \prime}=\frac{1}{\mathrm{e}^{\mathrm{i} 2 \theta_{v}}} \times \rho
$$

\subsection{Ehrenfest Paradox}

We show that the Lorentz transformations in graviton coordinates eliminate the Ehrenfest paradox. The Ehrenfest paradox is as follows. We consider a disk that rotates at velocity $v$. In the Lorentz transformations, since Lorentz contraction occurs only in the direction of motion, it occurs in the rotation direction, but it does not occur in the radial direction. For this reason, the circumference and diameter are expressed as follows.

$$
\begin{gathered}
\text { circumference }=2 \pi r \sqrt{1-\left(\frac{v}{c}\right)^{2}} . \\
\text { diameter }=2 r .
\end{gathered}
$$

From equations (17.22) and (17.23), when we divide the circumference by the diameter, it is expressed as

$$
\frac{\text { circumference }}{\text { diameter }}=\pi \sqrt{1-\left(\frac{v}{c}\right)^{2}} .
$$

Therefore, it is not $\pi$. This is the Ehrenfest paradox.

In the Lorentz transformations in graviton coordinates, Lorentz contraction occurs in all directions. For this reason, the circumference and diameter are expressed as follows.

$$
\begin{gathered}
\text { circumference }=2 \pi r \sqrt{1-\left(\frac{v}{\mathrm{c}}\right)^{2}} . \\
\text { diameter }=2 r \sqrt{1-\left(\frac{v}{\mathrm{c}}\right)^{2}} .
\end{gathered}
$$

From equations (17.25) and (17.26), when we divide the circumference by the diameter, it is expressed as

$$
\frac{\text { circumference }}{\text { diameter }}=\pi
$$

Therefore, it is $\pi$, and the Lorentz transformations in graviton coordinates eliminate the Ehrenfest paradox. 


\section{Law of Momentum Conservation and Square of Kinetic Energy}

In this section, from the quantized coordinate transformations in complex spacetime, we discuss the law of momentum conservation between different reference frames and show the square of kinetic energy based on momentum. We have already discussed the spin angular momentum and energy between different reference frames. We must discuss the momentum between different reference frames.

The individual elements in this section have the following meanings: The $t$ frame is the rest frame located at infinity from the source of gravity. The $t^{\prime}$ frame is the frame located at the radius $r$ from the source of gravity and the frame moving at a translational velocity $v^{\prime}$ against the $t$ frame. The velocity $v^{\prime}$ is the translational velocity of the $t^{\prime}$ frame observed from the $t$ frame. The velocity $v$ is the translational velocity of the $t^{\prime}$ frame observed from the rest frame located at the radius $r$ from the source of gravity.

\subsection{Quantized Coordinate Transformations in Complex Spacetime}

We show the quantized coordinate transformations in complex spacetime when considering the spacetime change caused by translational velocity and gravity. This complex spacetime is used to show the law of momentum conservation between different reference frames and the square of kinetic energy.

When considering the spacetime change caused by translational velocity and gravity, the velocity of radius (= the rotational speed) of the $t^{\prime}$ frame is expressed by equation (9.57), and the translational velocity of the $t^{\prime}$ frame is expressed by equation (9.56). For this reason, the speed of light of a complex number at this time is expressed as

$$
c^{\prime}=c \sqrt{1-\frac{r_{s}}{r}} \times \mathrm{e}^{\mathrm{i} \theta_{v}} .
$$

Therefore, the quantized coordinate transformations in complex spacetime at this time is expressed as

$$
\frac{r^{\prime}}{r}=\frac{\Delta r^{\prime}}{\Delta r}=\frac{t^{\prime}}{t}=\frac{\Delta t^{\prime}}{\Delta t}=\frac{c^{\prime}}{\mathrm{c}}=\sqrt{1-\frac{r_{s}}{r}} \times \mathrm{e}^{\mathrm{i} \theta_{v}} .
$$

Equation (18.2) is the quantized coordinate transformations in complex spacetime when an object is observed. From equations (14.21) and (18.2), the quantized coordinate transformations in complex spacetime when an object is not observed is expressed as

$$
\frac{r^{\prime}}{r}=\frac{\Delta r^{\prime}}{\Delta r}=\frac{t^{\prime}}{t}=\frac{\Delta t^{\prime}}{\Delta t}=\frac{c^{\prime}}{\mathrm{c}}=\sqrt{1-\frac{r_{s}}{r}} \times \mathrm{e}^{\mathrm{i} \theta_{v}} \times \mathrm{e}^{\mathrm{i} \theta_{w}} .
$$


The factors $\mathrm{e}^{\mathrm{i} \theta_{v}}$ and $\mathrm{e}^{\mathrm{i} \theta_{w}}$ of a complex number have the following characteristics.

The factor $\mathrm{e}^{\mathrm{i} \theta_{v}}$ is only represented by a complex number for convenience, and time does not advance to the past. The factor $\mathrm{e}^{\mathrm{i} \theta_{w}}$ is actually represented by a complex number, and time advances to the past.

The factor $\mathrm{e}^{\mathrm{i} \theta_{v}}$ represents the spacetime change caused by the translational motion of particles. The factor $\mathrm{e}^{\mathrm{i} \theta_{w}}$ represents the spacetime change caused by the gravity of waves.

In this way, the factors $\mathrm{e}^{\mathrm{i} \theta_{v}}$ and $\mathrm{e}^{\mathrm{i} \theta_{w}}$ of a complex number are paired factors with each other.

\subsection{Law of Momentum Conservation Between Different Reference Frames}

We show the law of momentum conservation between different reference frames. Since the energy of an electromagnetic wave is expressed as $E=p \mathrm{c}=\mathrm{h} f=m \mathrm{c}^{2}$, the momentum $p$ of the particle without active gravitational mass is expressed as $p=\frac{E}{\mathrm{c}}=m \mathrm{c}$. That is, the momentum is expressed as kinetic energy divided by the velocity of that kinetic energy. Therefore, we assume that the momentum is expressed as follows.

$$
\begin{gathered}
p^{\prime}=\frac{E^{\prime}}{\mathrm{c}}, \\
p_{r}^{\prime}=\frac{E_{r}^{\prime}}{\mathrm{c} \sqrt{1-\left(\frac{v}{\mathrm{c}}\right)^{2}}}, \\
p_{t}^{\prime}=\frac{E_{t}^{\prime}}{v},
\end{gathered}
$$

where $p^{\prime}, p_{r}^{\prime}$ and $p_{t}^{\prime}$ are the total momentum, momentum by the motion of radius and momentum by the translational motion when considering the spacetime change caused by translational velocity and gravity.

For particles that are not $E_{t}^{\prime}=0$, from equations (9.60) and (9.61) and (9.66), the momentums $p^{\prime}, p_{r}^{\prime}$ and $p_{t}^{\prime}$ are expressed as follows.

$$
\begin{aligned}
& p^{\prime}=\frac{m \mathrm{c} \sqrt{1-\frac{r_{s}}{r}}}{\sqrt{1-\left(\frac{v}{\mathrm{c}}\right)^{2}}} . \\
& p_{r}^{\prime}=m \mathrm{c} \sqrt{1-\frac{r_{s}}{r}} . \\
& p_{t}^{\prime}=\frac{m v \sqrt{1-\frac{r_{s}}{r}}}{\sqrt{1-\left(\frac{v}{\mathrm{c}}\right)^{2}}} .
\end{aligned}
$$


When considering the spacetime change caused by only translational velocity, we set the total momentum, momentum by the motion of radius and momentum by the translational motion as $p, p_{r}$ and $p_{t}$. Then, the momentums $p, p_{r}$ and $p_{t}$ are expressed as follows.

$$
\begin{gathered}
p=\frac{m \mathrm{c}}{\sqrt{1-\left(\frac{v}{\mathrm{c}}\right)^{2}}} . \\
p_{r}=m \mathrm{c} . \\
p_{t}=\frac{m v}{\sqrt{1-\left(\frac{v}{\mathrm{c}}\right)^{2}}} .
\end{gathered}
$$

From equations (18.10) to (18.12), the momentums $p^{\prime}, p_{r}^{\prime}$ and $p_{t}^{\prime}$ are expressed as follows.

$$
\begin{aligned}
& p^{\prime}=p \sqrt{1-\frac{r_{s}}{r}} . \\
& p_{r}^{\prime}=p_{r} \sqrt{1-\frac{r_{s}}{r}} . \\
& p_{t}^{\prime}=p_{t} \sqrt{1-\frac{r_{s}}{r}} .
\end{aligned}
$$

Similar to the speed of light of a complex number, the momentum $p_{o}^{\prime}$ in the complex spacetime of $\mathrm{e}^{\mathrm{i} \theta_{v}}$ is expressed as

$$
p_{o}^{\prime}=p^{\prime} \times \mathrm{e}^{\mathrm{i} \theta_{v}}=p_{r}^{\prime}+\mathrm{i} p_{t}^{\prime} .
$$

The square of the absolute value of the momentum $p_{o}^{\prime}$ is expressed as

$$
\left|p_{o}^{\prime}\right|^{2}=p^{2}=p_{r}^{\prime 2}+p_{t}^{\prime 2} .
$$

The momentum $p^{\prime}$ is the total momentum when belonging to the $t$ frame from the $t^{\prime}$ frame. The momentums $p_{r}^{\prime}$ and $p_{t}^{\prime}$ are momentums when belonging to the $t^{\prime}$ frame. Therefore, if we consider the momentum in the complex spacetime of $\mathrm{e}^{\mathrm{i} \theta_{v}}$, the law of momentum conservation between different reference frames holds. Importantly, since the rotational motion is equivalent to the motion of radius, we consider the angular momentum by the rotational motion as the momentum by the motion of radius. Then, since the momentum by the motion of radius and the momentum by the translational motion are vertical, their sum is conserved between different reference frames.

For particles that are $E_{t}^{\prime}=0$, from equation (18.6), $p_{t}^{\prime}=0$. Therefore, for particles that are $E_{t}^{\prime}=0$, the momentum $p^{\prime}$ of equation (18.17) is expressed as 


$$
p^{\prime}=p_{r}^{\prime} .
$$

From equation (10.35) and $p^{\prime}=p_{r}^{\prime}$, the momentums $p^{\prime}, p_{r}^{\prime}$ and $p_{t}^{\prime}$ of particles that are $E_{t}^{\prime}=0$ are expressed as follows.

$$
\begin{gathered}
p^{\prime}=m \mathrm{c} \sqrt{1-\frac{r_{s}}{r}} \sqrt{1-\left(\frac{v}{\mathrm{c}}\right)^{2}} . \\
p_{r}^{\prime}=m \mathrm{c} \sqrt{1-\frac{r_{s}}{r}} \sqrt{1-\left(\frac{v}{\mathrm{c}}\right)^{2}} . \\
p_{t}^{\prime}=0 .
\end{gathered}
$$

\subsection{Square of Kinetic Energy Based on Momentum}

We show the square of kinetic energy based on momentum. From equation (18.4), the kinetic energy $E^{\prime}$ is expressed as

$$
E^{\prime}=p^{\prime} \mathrm{c}
$$

From equations (18.16) and (18.22), the kinetic energy $E_{o}^{\prime}$ in the complex spacetime of $\mathrm{e}^{\mathrm{i} \theta_{v}}$ is expressed as

$$
E_{o}^{\prime}=E^{\prime} \times \mathrm{e}^{\mathrm{i} \theta_{v}}=p_{r}^{\prime} \mathrm{c}+\mathrm{i} p_{t}^{\prime} \mathrm{c} .
$$

The square of the absolute value of the kinetic energy $E_{o}^{\prime}$ is expressed as

$$
\left|E_{o}^{\prime}\right|^{2}=E^{\prime 2}=p^{\prime 2} c^{2}=p_{r}^{\prime 2} c^{2}+p_{t}^{\prime 2} \mathrm{c}^{2} .
$$

From equations (18.14) and (18.15), $E^{\prime 2}$ is expressed as

$$
E^{\prime 2}=p^{2} c^{2}=\left(1-\frac{r_{s}}{r}\right)\left(p_{r}^{2} c^{2}+p_{t}^{2} c^{2}\right)
$$

Therefore, the square of kinetic energy based on momentum is expressed by equation (18.25).

For particles that are not $E_{t}^{\prime}=0$, from $p_{r}=m \mathrm{c}$, equation (18.25) is expressed as

$$
E^{\prime 2}=p^{\prime 2} c^{2}=\left(1-\frac{r_{s}}{r}\right)\left(m^{2} c^{4}+p_{t}^{2} c^{2}\right)
$$

If $r=\infty$, equation (18.26) can be approximated as

$$
E^{\prime 2} \fallingdotseq p^{2} c^{2}=m^{2} c^{4}+p_{t}^{2} c^{2}
$$


Equation (18.27) is consistent with special relativity.

For particles that are $E_{t}^{\prime}=0$, from $p_{t}=0$, equation (18.25) is expressed as

$$
E^{\prime 2}=p^{\prime 2} \mathrm{c}^{2}=\left(1-\frac{r_{s}}{r}\right) p_{r}{ }^{2} \mathrm{c}^{2} .
$$

Note that equation (18.26) with $m=0$ is not consistent with equation (18.28).

\subsection{Dirac Equation and Klein-Gordon Equation in a Gravitational Field}

We show the Dirac equation and Klein-Gordon equation in a gravitational field. The Dirac equation and the Klein-Gordon equation are derived from the square of the kinetic energy of equation (18.27). The square of kinetic energy including the potential energy of gravity is expressed by equation (18.26).

The Klein-Gordon equation based on equation (18.26) is expressed as

$$
\frac{1}{c^{2}} \frac{\partial^{2} \psi}{\partial t^{2}}-\left(1-\frac{r_{s}}{r}\right) \nabla^{2} \psi+\frac{m^{2} c^{2}\left(1-\frac{r_{s}}{r}\right)}{\hbar^{2}} \psi=0 .
$$

The Dirac equation based on equation (18.26) is expressed as

$$
\mathrm{i \hbar} \frac{\partial \psi}{\partial t}=\left(-\mathrm{ich} \sqrt{1-\frac{r_{s}}{r}} \boldsymbol{\alpha} \cdot \nabla+\beta m \mathrm{c}^{2} \sqrt{1-\frac{r_{s}}{r}}\right) \psi,
$$

where $\boldsymbol{\alpha}$ and $\beta$ are matrices.

Therefore, the Dirac equation and Klein-Gordon equation in a gravitational field are expressed by equations (18.29) and (18.30).

\section{Bending of Light by Gravity, and Apsidal Precession}

In this section, we show the bending of light by gravity and the apsidal precession. The gravitational theory in this paper describes spacetime and gravity in a different way than the theory of relativity. For this reason, the bending of light by gravity and the apsidal precession must be shown in a different calculation. In this paper, they are calculated as follows. First, they are calculated by equations that do not consider the spacetime change. Second, in that calculation, we calculate the physical quantity of the $t^{\prime}$ frame by the time and distance of the $t$ frame. Then, they become the physical quantity of the $t^{\prime}$ frame observed from the $t$ frame. In this way, we show almost the same calculation result as the theory of relativity. 


\subsection{Bending of Light by Gravity}

We show the bending of light by gravity. We calculate the bending of light that does not consider the spacetime change, and then we calculate the bending of light that considers the spacetime change.

There is an object with mass $M$ at the origin $\mathrm{O}$. At $t=0$, light is located at coordinates $x=x, y=0, z=0$. The light moves in the $+y$-direction on the $X Y$ plane and receives gravity in the radial direction. Since light has no active gravitational mass, from equation (11.1), the gravity $F$ for light is expressed as $F=-\frac{2 \mathrm{G} M m}{r^{2}}$. When gravity $F$ is decomposed in the $x$-direction and the $y$-direction, they are expressed as follows.

$$
\begin{aligned}
& F_{x}=F \times \frac{x}{r}, \\
& F_{y}=F \times \frac{y}{r},
\end{aligned}
$$

where $F_{x}$ is the gravity in the $x$-direction, and $F_{y}$ is the gravity in the $y$-direction. For this reason, the equations of motion for light are expressed as follows.

$$
\begin{aligned}
& F_{x}=m \frac{\mathrm{d}^{2} x}{\mathrm{~d} t^{2}}=-\frac{2 \mathrm{G} M m}{r^{2}} \times \frac{x}{r} . \\
& F_{y}=m \frac{\mathrm{d}^{2} y}{\mathrm{~d} t^{2}}=-\frac{2 \mathrm{G} M m}{r^{2}} \times \frac{y}{r} .
\end{aligned}
$$

However, since we are considering the bending of light in the $x$-direction, we consider only the equation of motion of equation (19.3). From equation (19.3), the following equation is shown.

$$
\frac{\mathrm{d}^{2} x}{\mathrm{~d} t^{2}}=-\frac{2 \mathrm{G} M x}{r^{3}}
$$

Since the light moves on the $X Y$ plane, radius $r$ is expressed as

$$
r=\sqrt{x^{2}+y^{2}}
$$

Assuming that gravity is weak and the angle at which the light bends is extremely small, the motion of the light in the $y$-direction can be regarded as moving at the speed of light. Therefore, the motion of the light in the $y$-direction is expressed as

$$
y=\mathrm{c} t \text {. }
$$

From equations (19.6) and (19.7), radius $r$ is expressed as 


$$
r=\sqrt{x^{2}+\mathrm{c}^{2} t^{2}}
$$

From equation (19.8), equation (19.5) is expressed as

$$
\frac{\mathrm{d}^{2} x}{\mathrm{~d} t^{2}}=-\frac{2 \mathrm{G} M x}{\left(x^{2}+\mathrm{c}^{2} t^{2}\right)^{3 / 2}} .
$$

We set the bending angle $\theta$ of light as the angle from the straight line of $x=x$, where counterclockwise is positive. Then, the light moves in the $+y$-direction and $-x$-direction, so $\tan \theta$ is expressed as

$$
\tan \theta=-\frac{\mathrm{d} x}{\mathrm{~d} y}
$$

We assume that the bending angle $\theta$ of light is as close to 0 as possible. In that case, $\tan \theta$ can be approximated as

$$
\tan \theta \fallingdotseq \theta
$$

From equations (19.10) and (19.11), the bending angle $\theta$ of light is expressed as

$$
\theta \fallingdotseq \tan \theta=-\frac{\mathrm{d} x}{\mathrm{~d} y}
$$

The bending angle $\theta$ of light can be transformed as

$$
\theta=-\frac{\mathrm{d} x}{\mathrm{~d} y}=-\frac{\left(\frac{\mathrm{d} x}{\mathrm{~d} t}\right)}{\left(\frac{\mathrm{d} y}{\mathrm{~d} t}\right)} .
$$

From equation (19.7), the derivative $\frac{\mathrm{d} y}{\mathrm{~d} t}$ is expressed as

$$
\frac{\mathrm{d} y}{\mathrm{~d} t} \fallingdotseq \mathrm{c} .
$$

From equation (19.14), the bending angle $\theta$ of light is expressed as

$$
\theta=-\frac{\left(\frac{\mathrm{d} x}{\mathrm{~d} t}\right)}{\left(\frac{\mathrm{d} y}{\mathrm{~d} t}\right)}=-\frac{1}{\mathrm{c}} \frac{\mathrm{d} x}{\mathrm{~d} t} .
$$

Differentiating the bending angle $\theta$ of light with time $t$, it is expressed as

$$
\frac{\mathrm{d} \theta}{\mathrm{d} t}=-\frac{1}{\mathrm{c}} \frac{\mathrm{d}^{2} x}{\mathrm{~d} t^{2}}
$$

From equation (19.9), the derivative $\frac{\mathrm{d} \theta}{\mathrm{d} t}$ is expressed as 


$$
\frac{\mathrm{d} \theta}{\mathrm{d} t}=\frac{1}{\mathrm{c}} \frac{2 \mathrm{G} M x}{\left(x^{2}+\mathrm{c}^{2} t^{2}\right)^{3 / 2}} .
$$

Since the angle $\theta$ is the angle $\theta$ when integrated from $t=-\infty$ to $t=+\infty$, from equation (19.17), the bending angle $\theta$ of light is expressed as

$$
\theta=\frac{2 \mathrm{G} M}{\mathrm{c}} \int_{-\infty}^{+\infty} \frac{x}{\left(x^{2}+\mathrm{c}^{2} t^{2}\right)^{3 / 2}} \mathrm{~d} t .
$$

From $\theta \fallingdotseq 0$, we assume that $x$ in $\frac{\mathrm{d}^{2} x}{\mathrm{~d} t^{2}}$ is a constant $\mathrm{R}$.

$$
x=\mathrm{R} .
$$

From equation (19.19), the angle $\theta$ of equation (19.18) is expressed as

$$
\theta=\frac{2 \mathrm{G} M}{\mathrm{c}} \int_{-\infty}^{+\infty} \frac{\mathrm{R}}{\left(\mathrm{R}^{2}+\mathrm{c}^{2} t^{2}\right)^{3 / 2}} \mathrm{~d} t .
$$

When equation (19.20) is calculated, it is expressed as [19]

$$
\theta=\frac{4 \mathrm{G} M}{\mathrm{Rc}^{2}}
$$

Since we assume that light receives twice the normal gravity, the bending of light by gravity is naturally calculated as twice the normal bending. Therefore, the bending of light of equation (19.21) is consistent with the theory of relativity.

We show the bending of light that considers the spacetime change. Here, the $t$ frame is the rest frame located at infinity from the source of gravity, and the $t^{\prime}$ frame is the rest frame located at the radius $r$ from the source of gravity. When considering the spacetime change caused by gravity, the quantized coordinate transformations are expressed by equation (9.42), so the radius $r^{\prime}$ and $x^{\prime}$ of the $t^{\prime}$ frame are expressed as follows.

$$
\begin{aligned}
& r^{\prime}=r \sqrt{1-\frac{r_{s}}{r}} . \\
& x^{\prime}=x \sqrt{1-\frac{r_{s}}{r}} .
\end{aligned}
$$

When the physical quantity of the $t^{\prime}$ frame is calculated by the time and distance of the $t$ frame, it becomes the physical quantity of the $t^{\prime}$ frame observed from the $t$ frame. Moreover, in calculations other than kinetic energy and momentum, we transform the distance and time of the $t^{\prime}$ frame or the physical quantity related to them of the $t^{\prime}$ frame into the physical 
quantity observed from the $t$ frame. In the previous calculation, when we calculate like that, from equations (19.22) and (19.23), they are expressed as follows.

$$
\begin{aligned}
& F_{x}^{\prime}=m \frac{\mathrm{d}^{2} x^{\prime}}{\mathrm{d} t^{2}}=-\frac{2 \mathrm{G} M m}{r^{\prime 2}} \times \frac{x^{\prime}}{r^{\prime}}=-\frac{2 \mathrm{G} M m}{r^{2}\left(1-\frac{r_{s}}{r}\right)} \times \frac{x}{r}, \\
& \frac{\mathrm{d}^{2} x^{\prime}}{\mathrm{d} t^{2}}=-\frac{2 \mathrm{G} M x}{r^{3}\left(1-\frac{r_{s}}{r}\right)}, \\
& \theta^{\prime}=-\frac{\mathrm{d} x^{\prime}}{\mathrm{d} y}=-\frac{\left(\frac{\mathrm{d} x^{\prime}}{\mathrm{d} t}\right)}{\left(\frac{\mathrm{d} y}{\mathrm{~d} t}\right)}=-\frac{1}{\mathrm{c}} \frac{\mathrm{d} x^{\prime}}{\mathrm{d} t}, \\
& \frac{\mathrm{d} \theta^{\prime}}{\mathrm{d} t}=-\frac{1}{\mathrm{c}} \frac{\mathrm{d}^{2} x^{\prime}}{\mathrm{d} t^{2}}=\frac{1}{\mathrm{c}} \frac{2 \mathrm{G} M x}{\left(x^{2}+\mathrm{c}^{2} t^{2}\right)^{3 / 2}\left(1-\frac{r_{s}}{r}\right)}, \\
& \theta^{\prime}=\frac{2 \mathrm{G} M}{\mathrm{c}} \int_{-\infty}^{+\infty} \frac{\mathrm{R}}{\left(\mathrm{R}^{2}+\mathrm{c}^{2} t^{2}\right)^{3 / 2}\left(1-\frac{r_{s}}{r}\right)} \mathrm{d} t
\end{aligned}
$$

where $F_{x}^{\prime}$ is the gravity in the $x$-direction of the $t^{\prime}$ frame, and $\theta^{\prime}$ is the bending angle of light of the $t^{\prime}$ frame.

Assuming only a weak gravitational field of $\frac{r_{s}}{r}<<1$, equation (19.28) can be approximated as

$$
\theta^{\prime} \fallingdotseq \frac{2 \mathrm{G} M}{\mathrm{c}} \int_{-\infty}^{+\infty} \frac{\mathrm{R}}{\left(\mathrm{R}^{2}+\mathrm{c}^{2} t^{2}\right)^{3 / 2}}\left(1+\frac{r_{s}}{r}\right) \mathrm{d} t
$$

In equation (19.29), we set $\delta \theta$ as

$$
\delta \theta=\frac{2 \mathrm{G} M}{\mathrm{c}} \int_{-\infty}^{+\infty} \frac{\mathrm{R}}{\left(\mathrm{R}^{2}+\mathrm{c}^{2} t^{2}\right)^{3 / 2}} \times \frac{r_{s}}{r} \mathrm{~d} t .
$$

Then, from equations (19.20) and (19.30), equation (19.29) is expressed as

$$
\theta^{\prime}=\theta+\delta \theta
$$

Radius $r$ is expressed as

$$
r=\sqrt{\mathrm{R}^{2}+\mathrm{c}^{2} t^{2}} .
$$

For this reason, $\delta \theta$ is expressed as

$$
\delta \theta=\frac{2 \mathrm{G} M r_{s}}{\mathrm{c}} \int_{-\infty}^{+\infty} \frac{\mathrm{R}}{\left(\mathrm{R}^{2}+\mathrm{c}^{2} t^{2}\right)^{2}} \mathrm{~d} t
$$


When equation (19.33) is calculated, it is expressed as

$$
\delta \theta=\frac{G M}{\mathrm{Rc}^{2}} \times \frac{r_{s}}{\mathrm{R}} \times \pi .
$$

Therefore, from equations (19.21) and (19.34), $\theta^{\prime}$ is expressed as

$$
\theta^{\prime}=\frac{4 \mathrm{G} M}{\mathrm{Rc}^{2}}\left(1+\frac{1}{4} \times \frac{r_{s}}{\mathrm{R}} \times \pi\right) .
$$

Although the coordinates $\mathrm{R}=r_{s}$ do not satisfy the approximation condition, equation (19.35) at $\mathrm{R}=r_{s}=\frac{2 \mathrm{G} M}{\mathrm{c}^{2}}$ is expressed as

$$
\theta^{\prime}=2+\frac{\pi}{2}
$$

From equation (19.36), light bends approximately 180 degrees at the Schwarzschild radius. A 180-degree bend means that light cannot escape from a gravitational field. Therefore, equation (19.35) is physically valid. When considering the spacetime change caused by gravity, the bending of light by gravity increases by $\delta \theta$.

\subsection{Apsidal Precession}

Similar to the bending of light by gravity, we calculate planetary orbits that do not consider the spacetime change, and then we calculate planetary orbits that consider the spacetime change. From the planetary orbits that consider the spacetime change, we show apsidal precession.

As planetary orbits, we discuss the motion of the planet around mass $M$. With mass $M$ as the origin coordinate, the acceleration $a_{r}$ in the radius $r$-direction of polar coordinates and the acceleration $a_{\theta}$ in the angle $\theta$-direction of polar coordinates are expressed as follows.

$$
\begin{gathered}
a_{r}=\frac{\mathrm{d}^{2} r}{\mathrm{~d} t^{2}}-r\left(\frac{\mathrm{d} \theta}{\mathrm{d} t}\right)^{2} . \\
a_{\theta}=2 \frac{\mathrm{d} r}{\mathrm{~d} t} \frac{\mathrm{d} \theta}{\mathrm{d} t}+r \frac{\mathrm{d}^{2} \theta}{\mathrm{d} t^{2}}=\frac{1}{r} \frac{\mathrm{d}\left(r^{2} \frac{\mathrm{d} \theta}{\mathrm{d} t}\right)}{\mathrm{d} t} .
\end{gathered}
$$

Since the force received from mass $M$ is only radial gravity, the equation of motion for the planet is expressed as follows.

$$
\begin{gathered}
F_{r}=m a_{r}=m \frac{\mathrm{d}^{2} r}{\mathrm{~d} t^{2}}-m r\left(\frac{\mathrm{d} \theta}{\mathrm{d} t}\right)^{2}=-\frac{\mathrm{G} M m}{r^{2}} . \\
F_{\theta}=m a_{\theta}=\frac{m}{r} \frac{\mathrm{d}\left(r^{2} \frac{\mathrm{d} \theta}{\mathrm{d} t}\right)}{\mathrm{d} t}=0 .
\end{gathered}
$$


where $F_{r}$ is the force in the radius $r$-direction, and $F_{\theta}$ is the force in the angle $\theta$-direction. From equation (19.40), the following equation is shown.

$$
r^{2} \frac{\mathrm{d} \theta}{\mathrm{d} t}=\mathrm{D}
$$

where D is constant. From equation (19.41), the derivative $\frac{\mathrm{d} \theta}{\mathrm{d} t}$ is expressed as

$$
\frac{\mathrm{d} \theta}{\mathrm{d} t}=\frac{\mathrm{D}}{r^{2}}
$$

From equation (19.42), equation (19.39) is expressed as

$$
\frac{\mathrm{d}^{2} r}{\mathrm{~d} t^{2}}-\frac{\mathrm{D}^{2}}{r^{3}}=-\frac{\mathrm{G} M}{r^{2}} .
$$

Since radius $r$ can be regarded as a function of $\theta$ and $t$ from equation (19.41), when differentiating with $t$ with respect to $r$, it is expressed as

$$
\frac{\mathrm{d}}{\mathrm{d} t}=\frac{\mathrm{d}}{\mathrm{d} \theta} \frac{\mathrm{d} \theta}{\mathrm{d} t}=\frac{\mathrm{D}}{r^{2}} \frac{\mathrm{d}}{\mathrm{d} \theta} .
$$

From equation (19.44), the second derivative $\frac{\mathrm{d}^{2} r}{\mathrm{~d} t^{2}}$ is expressed as

$$
\frac{\mathrm{d}^{2} r}{\mathrm{~d} t^{2}}=\frac{\mathrm{D}^{2}}{r^{2}} \times \frac{\mathrm{d}\left(\frac{1}{r^{2}} \frac{\mathrm{d} r}{\mathrm{~d} \theta}\right)}{\mathrm{d} \theta}
$$

Substituting equation (19.45) into equation (19.43), it is expressed as

$$
\frac{\mathrm{D}^{2}}{r^{2}} \times \frac{\mathrm{d}\left(\frac{1}{r^{2}} \frac{\mathrm{d} r}{\mathrm{~d} \theta}\right)}{\mathrm{d} \theta}-\frac{\mathrm{D}^{2}}{r^{3}}=-\frac{\mathrm{G} M}{r^{2}} .
$$

We set the variable $u$ as

$$
u=\frac{1}{r}
$$

Then, the expression $\frac{1}{r^{2}} \frac{\mathrm{d} r}{\mathrm{~d} \theta}$ is expressed as

$$
\frac{1}{r^{2}} \frac{\mathrm{d} r}{\mathrm{~d} \theta}=-\frac{\mathrm{d} u}{\mathrm{~d} \theta}
$$

From equations (19.47) and (19.48), equation (19.46) is expressed as

$$
\frac{\mathrm{d}^{2} u}{\mathrm{~d} \theta^{2}}+u=\frac{\mathrm{G} M}{\mathrm{D}^{2}} .
$$

We set the constant $L$ with distance dimension as 


$$
L=\frac{\mathrm{D}^{2}}{\mathrm{G} M} .
$$

From equation (19.50), equation (19.49) is expressed as

$$
\frac{\mathrm{d}^{2} u}{\mathrm{~d} \theta^{2}}+u=\frac{1}{L} .
$$

Solving the differential equation of equation (19.51), it is expressed as

$$
u=C \cos \left(\theta+\theta_{0}\right)+\frac{1}{L},
$$

where $C$ and $\theta_{0}$ are constant of integration or arbitrary constant. From $u=\frac{1}{r}$, equation (19.52) is expressed as

$$
r=\frac{L}{1+L C \cos \left(\theta+\theta_{0}\right)} .
$$

For the sake of clarity, we set the arbitrary phase $\theta_{0}$ as $\theta_{0}=0$. We set the constant $e$ as

$$
e=L C=\frac{\mathrm{D}^{2} C}{\mathrm{G} M} .
$$

From equation (19.54) and $\theta_{0}=0$, equation (19.53) is expressed as [20]

$$
r=\frac{L}{1+e \cos \theta} .
$$

The radius $r$ of equation (19.55) is planetary orbits that do not consider the spacetime change. That is, this is planetary orbits in Newtonian mechanics. In this way, planetary orbits are derived.

We derive planetary orbits that consider the spacetime change. Here, the $t$ frame is the rest frame located at infinity from the source of gravity, and the $t^{\prime}$ frame is the frame located at the radius $r$ from the source of gravity and the frame moving at a translational velocity $v^{\prime}$ against the $t$ frame. When considering the spacetime change caused by translational velocity and gravity, the quantized coordinate transformations are expressed by equation (9.58), so the radius $r^{\prime}$ of the $t^{\prime}$ frame is expressed as

$$
r^{\prime}=r \sqrt{1-\left(\frac{v}{\mathrm{c}}\right)^{2}} \sqrt{1-\frac{r_{s}}{r}} .
$$

We calculate the velocity $v$ of $\sqrt{1-\left(\frac{v}{c}\right)^{2}}$. The speed $v$ in planetary orbits is the orbital speed. In the calculation of the orbital speed $v$, we assume that the spacetime change is not 
considered and planets are in uniform circular motion. The equation of motion for a planet in uniform circular motion is expressed as

$$
m a_{r}=-m \frac{v^{2}}{r}=-\frac{\mathrm{G} M m}{r^{2}},
$$

where $v$ is the orbital speed of the planet. From equation (19.57), the orbital speed $v$ is expressed as

$$
v=\sqrt{\frac{\mathrm{G} M}{r}} .
$$

From equation (19.58), the factor of $\sqrt{1-\left(\frac{v}{c}\right)^{2}}$ is expressed as

$$
\sqrt{1-\left(\frac{v}{c}\right)^{2}}=\sqrt{1-\frac{r_{s}}{2 r}}
$$

From equation (19.59) and $\left(\frac{r_{s}}{r}\right)^{2}<<1$, equation (19.56) is expressed as

$$
r^{\prime}=r \sqrt{1-\left(\frac{v}{\mathrm{c}}\right)^{2}} \sqrt{1-\frac{r_{s}}{r}}=r \sqrt{1-\frac{3 r_{s}}{2 r}+\frac{1}{2}\left(\frac{r_{s}}{r}\right)^{2}} \fallingdotseq r \sqrt{1-\frac{3 r_{s}}{2 r}} .
$$

When the physical quantity of the $t^{\prime}$ frame is calculated by the time and distance of the $t$ frame, it becomes the physical quantity of the $t^{\prime}$ frame observed from the $t$ frame. Moreover, in calculations other than kinetic energy and momentum, we transform the distance and time of the $t^{\prime}$ frame or the physical quantity related to them of the $t^{\prime}$ frame into the physical quantity observed from the $t$ frame. In the previous calculation, when we calculate like that, from equation (19.60), they are expressed as follows.

$$
\begin{gathered}
F_{r}^{\prime}=m a_{r}^{\prime}=m \frac{\mathrm{d}^{2} r^{\prime}}{\mathrm{d} t^{2}}-m r^{\prime}\left(\frac{\mathrm{d} \theta}{\mathrm{d} t}\right)^{2}=-\frac{\mathrm{G} M m}{r^{\prime 2}}, \\
F_{\theta}^{\prime}=m a_{\theta}^{\prime}=\frac{m}{r^{\prime}} \frac{\mathrm{d}\left(r^{\prime 2} \frac{\mathrm{d} \theta}{\mathrm{d} t}\right)}{\mathrm{d} t}=0, \\
r^{\prime 2} \frac{\mathrm{d} \theta}{\mathrm{d} t}=D^{\prime}, \\
\frac{\mathrm{d}}{\mathrm{d} t}=\frac{\mathrm{d}}{\mathrm{d} \theta} \frac{\mathrm{d} \theta}{\mathrm{d} t}=\frac{D^{\prime}}{r^{\prime 2}} \frac{\mathrm{d}}{\mathrm{d} \theta}, \\
\frac{\mathrm{d}^{2} r^{\prime}}{\mathrm{d} t^{2}}=\frac{D^{\prime 2}}{r^{\prime 2}} \times \frac{\mathrm{d}\left(\frac{1}{r^{\prime 2}} \frac{\mathrm{d} r^{\prime}}{\mathrm{d} \theta}\right)}{\mathrm{d} \theta},
\end{gathered}
$$




$$
\begin{gathered}
\frac{D^{\prime 2}}{r^{\prime 2}} \times \frac{\mathrm{d}\left(\frac{1}{r^{\prime 2}} \frac{\mathrm{d} r^{\prime}}{\mathrm{d} \theta}\right)}{\mathrm{d} \theta}-\frac{D^{\prime 2}}{r^{\prime 3}}=-\frac{\mathrm{G} M}{r^{\prime 2}}, \\
u^{\prime}=\frac{1}{r^{\prime}}, \\
\frac{1}{r^{\prime 2}} \frac{\mathrm{d} r^{\prime}}{\mathrm{d} \theta}=-\frac{\mathrm{d} u^{\prime}}{\mathrm{d} \theta}, \\
\frac{\mathrm{d}^{2} u^{\prime}}{\mathrm{d} \theta^{2}}+u^{\prime}=\frac{1}{L^{\prime}}, \\
L^{\prime}=\frac{D^{\prime 2}}{\mathrm{G} M},
\end{gathered}
$$

where $F_{r}^{\prime}$ is the force in the radius $r$-direction of the $t^{\prime}$ frame, and $F_{\theta}^{\prime}$ is the force in the angle $\theta$-direction of the $t^{\prime}$ frame. From equations (19.41) and (19.60) and (19.63), the constant $D^{\prime}$ is expressed as

$$
D^{\prime}=\mathrm{D}\left(1-\frac{3 r_{s}}{2 r}\right)
$$

From equations (19.50) and (19.70) and (19.71), the constant $L^{\prime}$ is expressed as

$$
L^{\prime}=\mathrm{L}\left(1-\frac{3 r_{s}}{2 r}\right)^{2}
$$

From equation (19.72), equation (19.69) is expressed as

$$
\frac{\mathrm{d}^{2} u^{\prime}}{\mathrm{d} \theta^{2}}+u^{\prime}=\frac{1}{\mathrm{~L}\left(1-\frac{3 r_{s}}{2 r}\right)^{2}} .
$$

From $\frac{3 r_{s}}{2 r}<<1$, equation (19.73) can be approximated as

$$
\frac{\mathrm{d}^{2} u^{\prime}}{\mathrm{d} \theta^{2}}+u^{\prime} \fallingdotseq \frac{1}{\mathrm{~L}}\left(1+\frac{3 r_{s}}{r}\right)
$$

Equation (19.74) cannot be solved because its variables ( $\frac{1}{r}=u$ and $\left.u^{\prime}\right)$ are different. For this reason, similar to the calculation of electromagnetic waves, we assume as

$$
u^{\prime}=\frac{1}{r^{\prime}}=u=\frac{1}{r}
$$

From equation (19.75), equation (19.74) is expressed as 


$$
\frac{\mathrm{d}^{2} u^{\prime}}{\mathrm{d} \theta^{2}}+\left(1-\frac{3 r_{s}}{L}\right) u^{\prime}=\frac{1}{\mathrm{~L}}
$$

Solving the differential equation of equation (19.76), it is expressed as

$$
u^{\prime}=C \cos \left(\sqrt{1-\frac{3 r_{s}}{L}} \theta+\theta_{0}\right)+\frac{1}{L\left(1-\frac{3 r_{s}}{L}\right)},
$$

where $C$ and $\theta_{0}$ are constant of integration or arbitrary constant. From $u^{\prime}=\frac{1}{r^{\prime}}$, equation (19.77) is expressed as

$$
r^{\prime}=\frac{L\left(1-\frac{3 r_{s}}{L}\right)}{1+L C\left(1-\frac{3 r_{s}}{L}\right) \cos \left(\sqrt{1-\frac{3 r_{s}}{L}} \theta+\theta_{0}\right)} .
$$

For the sake of clarity, we set the arbitrary phase $\theta_{0}$ as $\theta_{0}=0$. From $e=C L$ and $\theta_{0}=0$, equation (19.78) is expressed as

$$
r^{\prime}=\frac{L\left(1-\frac{3 r_{s}}{L}\right)}{1+e\left(1-\frac{3 r_{s}}{L}\right) \cos \left(\sqrt{1-\frac{3 r_{s}}{L}} \theta\right)} .
$$

The radius $r^{\prime}$ of equation (19.79) is planetary orbits that consider the spacetime change, and all are the same as planetary orbits that do not consider the spacetime change except for the element $-\frac{3 r_{s}}{L}$. That is, ignoring the element $-\frac{3 r_{s}}{L}$ in $L$ and $e$, planetary orbits change only in the angular component.

We derive the apsidal precession when a planet makes one revolution. Since the angle of one revolution is $2 \pi \mathrm{rad}$, the angle $\theta$ at that time is expressed as

$$
\theta=\frac{2 \pi}{\sqrt{1-\frac{3 r_{s}}{L}}}
$$

From $\frac{3 r_{s}}{L}<<1$, the angle $\theta$ can be approximated as

$$
\theta=\frac{2 \pi}{\sqrt{1-\frac{3 r_{s}}{L}}} \fallingdotseq 2 \pi\left(1+\frac{3 r_{s}}{2 L}\right) .
$$

Therefore, when a planet makes one revolution, the apsidal precession $\delta \theta$ is expressed as

$$
\delta \theta=\theta-2 \pi=2 \pi \frac{3 r_{s}}{2 L}
$$

The semi-major axis $R$ is expressed as 


$$
R=\frac{L}{1-e^{2}}
$$

From equation (19.83), the apsidal precession $\delta \theta$ is expressed as

$$
\delta \theta=2 \pi \frac{3 r_{s}}{2 L}=\frac{6 \pi \mathrm{G} M}{\mathrm{c}^{2} R\left(1-e^{2}\right)} .
$$

The apsidal precession $\delta \theta$ of equation (19.84) is consistent with the theory of relativity. Therefore, planetary orbits $r^{\prime}$ are different from the theory of relativity, but the apsidal precession $\delta \theta$ is consistent with the theory of relativity. From the calculation process, 1/3 of the apsidal precession $\delta \theta$ is the effect of spacetime change caused by translational velocity, and $2 / 3$ of that is the effect of spacetime change caused by gravity.

In summary, in this paper, the bending of light by gravity and the apsidal precession are calculated as follows. First, they are calculated by equations that do not consider the spacetime change. Second, in that calculation, we calculate the physical quantity of the $t^{\prime}$ frame by the time and distance of the $t$ frame. Then, they become the physical quantity of the $t^{\prime}$ frame observed from the $t$ frame. In this way, almost the same calculation results as the theory of relativity are shown.

\section{Gravitoelectromagnetism Equations of This Paper}

We discuss the gravitoelectromagnetism (GEM) equations of this paper. From the observation of gravitational waves and section 19, the gravitational theory in this paper requires equations without spacetime change to explain gravitational waves. We assume that their equations are the GEM equations. However, from section 7, three forces received by mass exist. For this reason, we add expressions to the GEM equations.

\subsection{Gauss's Law for the GEM Equations of This Paper}

We show Gauss's law for the GEM equations of this paper. From equations (2.2) and (7.24) and divergence theorem, forces $F$ received by mass are expressed as

$$
F=\int_{S} \vec{P} \cdot \mathrm{d} \vec{S}=\int_{V} \nabla \cdot \vec{P} \mathrm{~d} V=-\frac{\mathrm{G} M m}{r^{2}}\left(1+\frac{r}{\mathrm{r}_{\mathrm{e}}}-\frac{A_{P} r^{3}}{\mathrm{G} M}\right),
$$

where $V$ is the volume surrounded by closed area $S$. Mass $M$ is expressed as

$$
M=\int \rho_{g} \mathrm{~d} V
$$


where $\rho_{g}$ is the mass density of mass $M$. From equation (20.2), equation (20.1) is expressed as

$$
\nabla \cdot \vec{P}=-\frac{\mathrm{G} m \rho_{g}}{r^{2}}\left(1+\frac{r}{\mathrm{r}_{\mathrm{e}}}-\frac{A_{P} r^{3}}{\mathrm{G} M}\right) .
$$

From $F=P \times S$, multiplying equation (20.3) by the area $S=4 \pi r^{2}$, it is expressed as

$$
\nabla \cdot \vec{F}=-4 \pi \mathrm{G} m \rho_{g}\left(1+\frac{r}{\mathrm{r}_{\mathrm{e}}}-\frac{A_{P} r^{3}}{\mathrm{G} M}\right)
$$

Similar to the electromagnetic field, we define the gravitoelectric field $\vec{E}_{g}$ as

$$
\vec{E}_{g}=\frac{\vec{F}}{m}
$$

From equation (20.5), equation (20.4) is expressed as

$$
\nabla \cdot \vec{E}_{g}=-4 \pi \mathrm{G} \rho_{g}\left(1+\frac{r}{\mathrm{r}_{\mathrm{e}}}-\frac{A_{P} r^{3}}{\mathrm{G} M}\right)
$$

When considering at the current time, from equation (7.19), $A_{P}$ is expressed as

$$
A_{P} \fallingdotseq \frac{\mathrm{G} M}{\mathrm{r}_{\mathrm{e}} \times r_{e 2}^{2}}
$$

From equation (20.7), equation (20.6) is expressed as

$$
\nabla \cdot \overrightarrow{E_{g}}=-4 \pi \mathrm{G} \rho_{g}\left(1+\frac{r}{\mathrm{r}_{\mathrm{e}}}-\frac{r^{3}}{\mathrm{r}_{\mathrm{e}} \times r_{e 2}^{2}}\right) .
$$

In the case of $r<<\mathrm{r}_{\mathrm{e}}<<r_{e 2}$, equation (20.8) can be approximated as

$$
\nabla \cdot \overrightarrow{E_{g}} \fallingdotseq-4 \pi \mathrm{G} \rho_{g} .
$$

Therefore, in the case of $r<<\mathrm{r}_{\mathrm{e}}<<r_{e 2}$, equation (20.8) is consistent with Gauss's law for the GEM equations.

\subsection{GEM Equations of This Paper}

We show the reason why the gravitational theory in this paper adopts the GEM equations, and then we show the GEM equations of this paper.

First, the gravitational theory in this paper requires equations without spacetime change to explain gravitational waves. 
Second, the weak gravitational field approximation in general relativity is expressed as the GEM equations [21, 22].

Third, like photons, gravitons are composed only of spacetime particles.

Fourth, from section 10, photons propagate through the medium of gravitons. That is, in the space where photons propagate, gravitons must also exist at the same time. This suggests that photons always exist in pairs with gravitons.

From these, we conclude that gravitational fields are described by the GEM equations, which is a form similar to Maxwell's equations. Even if there is a problem with the GEM equations, for the reasons mentioned above, the gravitational theory in this paper has no choice but to adopt the GEM equations.

When not considering spacetime change, from equation (20.6) and the GEM equations, gravitational field equations in this paper are expressed as follows.

$$
\begin{gathered}
\nabla \times \vec{E}_{g}=-\frac{\partial \overrightarrow{B_{g}}}{\partial t} \\
\nabla \times \overrightarrow{B_{g}}=\frac{1}{\mathrm{c}^{2}} \frac{\partial \vec{E}_{g}}{\partial t}-\frac{4 \pi \mathrm{G}}{\mathrm{c}^{2}} \overrightarrow{j_{g}} \\
\nabla \cdot \vec{E}_{g}=-4 \pi \mathrm{G} \rho_{g}\left(1+\frac{r}{\mathrm{r}_{\mathrm{e}}}-\frac{A_{P} r^{3}}{\mathrm{GM}}\right), \\
\nabla \cdot \overrightarrow{B_{g}}=0
\end{gathered}
$$

where $\overrightarrow{B_{g}}$ is the gravitomagnetic field, and $\overrightarrow{j_{g}}$ is mass current density and is expressed as

$$
\overrightarrow{j_{g}}=\rho_{g} \times \overrightarrow{v_{g}}
$$

where $\overrightarrow{v_{g}}$ is the velocity of the mass flow.

\subsection{Invariance of GEM Equations of This Paper Under the Lorentz Transformations in Graviton Coordinates}

We show the invariance of GEM equations of this paper under the Lorentz transformations in graviton coordinates. Here, the $t$ frame is the rest frame, the $t^{\prime}$ frame is the frame moving at a translational velocity $v$ against the $t$ frame.

We transform the physical quantities of the $t^{\prime}$ frame based on the Lorentz transformations in graviton coordinates. In the following, the elements $t, \overrightarrow{E_{g}}, \overrightarrow{B_{g}}, \overrightarrow{j_{g}}, \rho_{g}$ and $\nabla$ are the elements of the $t$ frame, and the elements $t^{\prime}, \overrightarrow{E_{g}^{\prime}}, \overrightarrow{B_{g}^{\prime}}, \overrightarrow{j_{g}^{\prime}}, \rho_{g}^{\prime}$ and $\nabla^{\prime}$ are the elements of the $t^{\prime}$ frame.

Similar to Maxwell's equations, the physical quantities of the $t^{\prime}$ frame are transformed as follows. 


$$
\begin{gathered}
\frac{\partial}{\partial t^{\prime}}=\frac{1}{\mathrm{e}^{\mathrm{i} \theta_{v}}} \times \frac{\partial}{\partial t} . \\
\nabla^{\prime}=\frac{1}{\mathrm{e}^{\mathrm{i} \theta_{v}}} \times \nabla . \\
\overrightarrow{E_{g}^{\prime}}=\frac{1}{\mathrm{e}^{\mathrm{i} 2 \theta_{v}}} \times \overrightarrow{E_{g}} . \\
\overrightarrow{B_{g}^{\prime}}=\frac{1}{\mathrm{e}^{\mathrm{i} 2 \theta_{v}}} \times \overrightarrow{B_{g}} . \\
\rho_{g}^{\prime}=\frac{1}{\mathrm{e}^{\mathrm{i} 3 \theta_{v}}} \times \rho_{g} . \\
\overrightarrow{j_{g}^{\prime}}=\frac{1}{\mathrm{e}^{\mathrm{i} 3 \theta_{v}}} \times \overrightarrow{j_{g}} . \\
A_{p}^{\prime}=\frac{1}{\mathrm{e}^{\mathrm{i} 3 \theta_{v}}} \times A_{p} .
\end{gathered}
$$

From $A_{P} \fallingdotseq \frac{\mathrm{G} M}{\mathrm{r}_{\mathrm{e}} \times r_{e 2}^{2}}$, the parameter $A_{P}^{\prime}$ is expressed by equation (20.18).

We show the invariance of GEM equations of this paper under the Lorentz transformations in graviton coordinates. The GEM equations of this paper of the $t^{\prime}$ frame are shown as follows.

$$
\begin{gathered}
\nabla^{\prime} \times \overrightarrow{E_{g}^{\prime}}=-\frac{\partial \overrightarrow{B_{g}^{\prime}}}{\partial t^{\prime}} . \\
\nabla^{\prime} \times \overrightarrow{B_{g}^{\prime}}=\frac{1}{\mathrm{c}^{2}} \frac{\partial \overrightarrow{E_{g}^{\prime}}}{\partial t^{\prime}}-\frac{4 \pi \mathrm{G}}{\mathrm{c}^{2}} \overrightarrow{j_{g}^{\prime}} . \\
\nabla^{\prime} \cdot \overrightarrow{E_{g}^{\prime}}=-4 \pi \mathrm{G} \rho_{g}^{\prime}\left(1+\frac{r^{\prime}}{r_{e}^{\prime}}-\frac{A_{P}^{\prime} r^{\prime 3}}{\mathrm{G} M}\right) . \\
\nabla^{\prime} \cdot \overrightarrow{B_{g}^{\prime}}=0 .
\end{gathered}
$$

Transforming the individual elements in these equations, they are expressed as follows.

$$
\begin{gathered}
\frac{1}{\mathrm{e}^{\mathrm{i} 3 \theta_{v}}} \times\left(\nabla \times \overrightarrow{E_{g}}\right)=\frac{1}{\mathrm{e}^{\mathrm{i} 3 \theta_{v}}} \times\left(-\frac{\partial \overrightarrow{B_{g}}}{\partial t}\right) . \\
\frac{1}{\mathrm{e}^{\mathrm{i} 3 \theta_{v}}} \times\left(\nabla \times \overrightarrow{B_{g}}\right)=\frac{1}{\mathrm{e}^{\mathrm{i} 3 \theta_{v}}} \times\left(\frac{1}{\mathrm{c}^{2}} \frac{\partial \overrightarrow{E_{g}}}{\partial t}-\frac{4 \pi \mathrm{G}}{\mathrm{c}^{2}} \overrightarrow{j_{g}}\right) .
\end{gathered}
$$




$$
\begin{gathered}
\frac{1}{\mathrm{e}^{\mathrm{i} 3 \theta_{v}} \times\left(\nabla \cdot \overrightarrow{E_{g}}\right)=}=\frac{1}{\mathrm{e}^{\mathrm{i} 3 \theta_{v}}} \times\left(-4 \pi \mathrm{G} \rho_{g}\left(1+\frac{r}{\mathrm{r}_{\mathrm{e}}}-\frac{A_{P} r^{3}}{\mathrm{G} M}\right)\right) . \\
\frac{1}{\mathrm{e}^{\mathrm{i} 3 \theta_{v}}} \times\left(\nabla \cdot \overrightarrow{B_{g}}\right)=0 .
\end{gathered}
$$

Therefore, they are transformed to GEM equations of this paper of the $t$ frame, and GEM equations of this paper are invariant under the Lorentz transformations in graviton coordinates. Note that the GEM equations of this paper are invariant under arbitrary quantized coordinate transformations.

\subsection{GEM Equations of This Paper of the $t^{\prime}$ Frame Observed From the $t$ Frame and Gravitational Waves of That}

We show the GEM equations of this paper of the $t^{\prime}$ frame observed from the $t$ frame and the gravitational waves of that. Here, the $t$ frame is the rest frame, the $t^{\prime}$ frame is the frame moving at a translational velocity $v$ against the $t$ frame.

We show the wave equations of gravitational waves. When gravitational waves propagate through a vacuum, from $\rho_{g}=0$, equation (20.6) is expressed as

$$
\nabla \cdot \vec{E}_{g}=0
$$

and from $\overrightarrow{j_{g}}=\overrightarrow{0}$, equation (20.11) is expressed as

$$
\nabla \times \overrightarrow{B_{g}}=\frac{1}{\mathrm{c}^{2}} \frac{\partial \vec{E}_{g}}{\partial t} .
$$

Then, the GEM equations of this paper have the almost same form as Maxwell's equations, so the wave equations of gravitational waves are expressed as follows.

$$
\begin{aligned}
\nabla^{2} \vec{E}_{g} & =\frac{1}{\mathrm{c}^{2}} \frac{\partial^{2} \vec{E}_{g}}{\partial t^{2}} . \\
\nabla^{2} \vec{B}_{g} & =\frac{1}{\mathrm{c}^{2}} \frac{\partial^{2} \overrightarrow{B_{g}}}{\partial t^{2}} .
\end{aligned}
$$

We show the gravitational waves of the $t^{\prime}$ frame observed from the $t$ frame. Similar to electromagnetic waves, the wave equations of their gravitational waves are expressed as follows.

$$
\nabla^{2}{\overrightarrow{E_{g}^{\prime}}}_{g}=\frac{1}{\left(\mathrm{c} \times \mathrm{e}^{\mathrm{i} \theta_{v}}\right)^{2}} \frac{\partial^{2} \vec{E}_{g}}{\partial t^{2}} .
$$




$$
\nabla^{2} \overrightarrow{B_{g}^{\prime}}=\frac{1}{\left(\mathrm{c} \times \mathrm{e}^{\mathrm{i} \theta_{v}}\right)^{2}} \frac{\partial^{2} \overrightarrow{B_{g}}}{\partial t^{2}} .
$$

From equations (20.31) and (20.32), the light speed $c^{\prime}$ of the gravitational waves of the $t^{\prime}$ frame observed from the $t$ frame is expressed as

$$
c^{\prime}=\mathrm{c} \times \mathrm{e}^{\mathrm{i} \theta_{v}} .
$$

Therefore, the speed of light of gravitational waves is expressed in the same way as the speed of light of electromagnetic waves. For this reason, the speed of light of the quantized coordinate transformations and electromagnetic waves and gravitational waves are all the same.

Similar to Maxwell's equations, the GEM equations of this paper of the $t^{\prime}$ frame observed from the $t$ frame are expressed as follows.

$$
\begin{gathered}
\nabla \times \overrightarrow{E_{g}^{\prime}}=\frac{1}{\mathrm{e}^{\mathrm{i} 2 \theta_{v}}} \times\left(-\frac{\partial \overrightarrow{B_{g}}}{\partial t}\right) \\
\nabla \times \overrightarrow{B_{g}^{\prime}}=\frac{1}{\mathrm{e}^{\mathrm{i} 2 \theta_{v}}} \times\left(\frac{1}{\mathrm{c}^{2}} \frac{\partial \overrightarrow{E_{g}}}{\partial t}-\frac{4 \pi \mathrm{G}}{\mathrm{c}^{2}} \overrightarrow{j_{g}}\right) . \\
\nabla \cdot \overrightarrow{E_{g}^{\prime}}=\frac{1}{\mathrm{e}^{\mathrm{i} 2 \theta_{v}}} \times\left(-4 \pi \mathrm{G} \rho_{g}\left(1+\frac{r}{\mathrm{r}_{\mathrm{e}}}-\frac{A_{P} r^{3}}{\mathrm{G} M}\right)\right) . \\
\nabla \cdot \overrightarrow{B_{g}^{\prime}}=0 .
\end{gathered}
$$

From these, the mass current density of the $t^{\prime}$ frame observed from the $t$ frame and the mass density of that are expressed as follows.

$$
\begin{aligned}
& \overrightarrow{j_{g}^{\prime \prime}}=\frac{1}{\mathrm{e}^{\mathrm{i} 2 \theta_{v}}} \times \vec{j}_{g} . \\
& \rho_{g}^{\prime \prime}=\frac{1}{\mathrm{e}^{\mathrm{i} 2 \theta_{v}}} \times \rho_{g} .
\end{aligned}
$$

\section{Conclusion}

In this paper, starting from the assumption of equivalence of energy and mass and spacetime, we studied macroscopic and microscopic gravitational phenomena in terms of thermodynamics. Spacetime is quantized by spacetime particles with the minimum mass $m_{s p}$, and spacetime is a group of spacetime particles. The minimum mass $m_{s p}$ is $m_{s p}=1.05 \times 10^{-62} \times 4 \pi s \mathrm{~kg}$, 
where $s$ is the spin quantum number of matter. Dark matter is spacetime particles composing spacetime. Gravity can be described as not only Newton's law of universal gravity but also a thermodynamic pressure caused by spacetime particles. From this, the equation of state of spacetime particles is derived.

Active gravitational mass generates the spacetime particle number density around an object. If an object has active gravitational mass, an inertial force by the spacetime particle number density works on the object. If an object does not have active gravitational mass, an inertial force by the spacetime particle number density does not work on the object. Even if particles do not have active gravitational mass and are moving at the speed of light, they have passive gravitational mass and an inertial mass, and they have kinetic energy by the inertial mass and potential energy of gravity by passive gravitational mass, and they receive gravity.

Spacetime particles are spherical surface particles that are hollow and distributed only on the surface of the sphere, and they are harmonic oscillators by the thermal vibration of the dimensionless temperature $T_{s p}$. Spacetime structure is the quantized 3-dimensional sphere of Poincaré conjecture. Spacetime or gravitational fields are a group of harmonic oscillators of spacetime particles. The quantized minimum distance $\Delta r$ and the quantized minimum time $\Delta t$ are based on the uncertainty principle. From the quantized spacetime, the quantized graviton coordinates are derived. In the minimum space in graviton coordinates, even if an object touches a certain point in the space, the object touches the entire space. The generally recognized spacetime is the space where gravitons exist. In the space without gravitons, gravity and an inertial force do not work, time and space cannot be quantized and cannot be counted, and the entire space becomes one minimum spacetime. For this reason, even in the space without gravitons, it is not that there is no spacetime, and the entire space is one minimum spacetime. All physical quantities related to energy and mass and spacetime are quantized by spacetime particles. The minimum radius is expressed as $\frac{\Delta r}{2}=\frac{\lambda}{2 \pi} \times s$, where $\lambda$ is the Compton wavelength, and $s$ is the spin quantum number. An elementary particle is composed of spacetime particles with kinetic energy and charged particles with charges and potential spacetime particles with potential energy.

The expansion source of spacetime is at the center of individual matter with active gravitational mass. The radius of space includes the spacetime radius and the inter-matter radius. The spacetime radius is the radius from the center of the matter of the expansion source to spacetime particles without active gravitational mass. The inter-matter radius is the radius from the center of the matter of the expansion source to another matter with active gravitational mass. The expansion speed of the spacetime radius is usually the speed of light. The expansion speed of the inter-matter radius is usually less than the speed of light. The expansion we are observing is the expansion of the inter-matter radius. However, since the expansion of the inter-matter radius is caused by the expansion of the spacetime radius, it is not a mistake to refer to the expansion of the inter-matter radius as the expansion of 
spacetime. Changes in the expansion speed are caused by changes in the mass of ordinary matter. Dark energy that accelerates the expansion of spacetime is a virtual negative mass by the mass reduction of ordinary matter.

There are three forces received by mass. The first is the gravity of ordinary matter, which is inversely proportional to the square of distance. The second is the gravity of spacetime particles (generally dark matter), which is inversely proportional to distance. The third is the spacetime expansion acceleration force (generally dark energy), which is proportional to distance.

From the spin angular momentum or the equation of state of spacetime particles, the quantized coordinate transformations are derived. The quantized coordinate transformations hold only in the quantized graviton coordinates. The rotational velocity of spacetime particles and the velocity of the radius of spacetime particles are physically equivalent. Changes in the expansion speed of spacetime are derived from the quantized coordinate transformations.

The square of the absolute value of a wave function represents the spacetime particle number density within an elementary particle. Wave function reduction is the following phenomenon. Before observation, the spacetime particle distribution within an elementary particle expands to the minimum radius $\frac{\Delta r}{2}$ or more, but when observed, the distribution contracts to the minimum radius $\frac{\Delta r}{2}$ around the observation point at superluminal speed. From the quantized coordinate transformations, the speed of wave function reduction is derived as a speed that far exceeds the speed of light.

From the law of energy conservation between different reference frames, the spacetime change and the kinetic energy are derived. Light has only rotational kinetic energy and no translational kinetic energy. The medium of light is spacetime particles composing spacetime. Light propagates through a medium that moves with the observer. The speed of light is not the translational speed but the speed of radius. The light belonging to the rest frame perceives time and space because its translational velocity $v$ is zero $(v=0)$.

There are potential spacetime particles with the potential energy of $U^{\prime}=m_{s p} \mathrm{c}^{2}$ and spacetime particles with the kinetic energy of $E=m_{s p} c^{2}$. Since potential spacetime particles have no kinetic energy, they are virtual particles that cannot be observed directly. The potential black hole elementary particle is composed of potential spacetime particles, and it exists at the center of $r=0$ within an elementary particle. The potential energy of $U^{\prime}=m_{s p} c^{2}$ is converted into the kinetic energy of $E=m_{s p} c^{2}$, and the potential spacetime particle turns into the spacetime particle. In this way, the law of energy conservation holds in the production of spacetime particles.

Before the birth of the universe, there was one black hole elementary particle with the minimum radius $\frac{\Delta r}{2}$ in the space without gravitons, and there were charged particles and potential black hole elementary particles within the black hole elementary particle. The potential black hole elementary particle emits spacetime particles, the black hole elementary particle absorbs them by gravity, and its mass increases. For this reason, before the birth 
of the universe, spacetime was repeatedly created and annihilated, and spacetime was an imaginary spacetime.

In that process, the mass $M_{p}$ of the potential black hole elementary particle decreases, and the mass $M$ of the black hole elementary particle increases. When the mass $M$ of the black hole elementary particle is $M>M_{p}$, the spacetime particle is produced at $r=2 \Delta r$ and turns into the potential spacetime particle. That potential spacetime particle moves to $r=\Delta r$ from $r=2 \Delta r$ and turns into the spacetime particle at $r=\Delta r$. At this time, the black hole elementary particle collapses by the gravity of the repulsive force of itself, and individual matter is produced. The elementary particle radius of the individual matter is much larger than the elementary particle radius of the black hole elementary particle with the total mass of ordinary matter. The change of the elementary particle radius to $\frac{\Delta r}{2}$ occurs at a speed faster than the speed of light by wave function reduction. In the case of inflation, since the elementary particle radius expands to $\frac{\Delta r}{2}$, that change is wave function enlargement. That is, inflation is a phenomenon in which each elementary particle radius expands from less than $\frac{\Delta r}{2}$ to $\frac{\Delta r}{2}$ at the same speed as wave function reduction.

When an elementary particle is not observed, the spacetime of the elementary particle is complex spacetime represented as the sum of real spacetime and imaginary spacetime. Real spacetime is caused by the elementary particle composed of spacetime particles. Imaginary spacetime is caused by the potential black hole elementary particle composed of potential spacetime particles. More specifically, imaginary spacetime is caused by the factor $\sqrt{1-\frac{r_{s}}{r}}$, which is the spacetime change caused by the gravity of the potential black hole elementary particle.

If spacetime is a real spacetime, time advances to the future, and if spacetime is an imaginary spacetime, time advances to the past. However, when time advances to the past and spacetime is annihilated, time advances to the future. Therefore, in imaginary spacetime, spacetime is repeatedly created and annihilated. The time in complex spacetime advances to the past at the same time as it advances to the future. The time when not observed is a complex time, time advances to the past at the same time as it advances to the future.

The Lorentz transformations in graviton coordinates are expressed as $\frac{r^{\prime}}{r}=\frac{\Delta r^{\prime}}{\Delta r}=\frac{t^{\prime}}{t}=$ $\frac{\Delta t^{\prime}}{\Delta t}=\frac{c^{\prime}}{\mathrm{c}}=\mathrm{e}^{\mathrm{i} \theta_{v}}=\sqrt{1-\left(\frac{v}{\mathrm{c}}\right)^{2}}+\mathrm{i} \frac{v}{\mathrm{c}}$. Their real part is the time, distance and velocity for the motion of radius or the rotational motion or the motion within the $t^{\prime}$ frame. Their imaginary part is the time, distance and velocity for the translational motion or the motion of the entire $t^{\prime}$ frame. When $v \fallingdotseq \mathrm{c}$, the motion within the $t^{\prime}$ frame stops, but the motion of the entire $t^{\prime}$ frame moves at almost the speed of light, and the time $\operatorname{Im}\left(t^{\prime}\right)$ of the entire $t^{\prime}$ frame has elapsed. Maxwell's equations are invariant under the Lorentz transformations in graviton coordinates. In the Lorentz transformations in graviton coordinates, Lorentz contraction occurs in all directions, so the Ehrenfest paradox is eliminated.

If we consider the momentum in the complex spacetime of $\mathrm{e}^{\mathrm{i} \theta_{v}}$, the law of momentum conservation between different reference frames holds. The square of kinetic energy based 
on momentum is expressed as $E^{\prime 2}=p^{\prime 2} c^{2}=\left(1-\frac{r_{s}}{r}\right)\left(p_{r}{ }^{2} c^{2}+p_{t}{ }^{2} c^{2}\right)$, where $p^{\prime}$ is the total momentum, $p_{r}$ is momentum by the motion of radius, $p_{t}$ is momentum by the translational motion. Since light has no translational kinetic energy, the $p_{t}$ of light is $p_{t}=0$. For this reason, the square of kinetic energy for light is expressed as $E^{\prime 2}=p^{\prime 2} c^{2}=\left(1-\frac{r_{s}}{r}\right) p_{r}{ }^{2} c^{2}$.

The bending of light by gravity and the apsidal precession are calculated as follows. First, they are calculated by equations that do not consider the spacetime change. Second, in that calculation, we calculate the physical quantity of the $t^{\prime}$ frame by the time and distance of the $t$ frame. Then, they become the physical quantity of the $t^{\prime}$ frame observed from the $t$ frame. In this way, almost the same calculation results as the theory of relativity are shown.

The gravitational theory in this paper requires equations without spacetime change to explain gravitational waves. We assume that their equations are the GEM equations. However, three forces received by mass exist. For this reason, we add expressions to the GEM equations, and Gauss's law in the GEM equations of this paper is expressed as $\nabla \cdot \overrightarrow{E_{g}}=-4 \pi \mathrm{G} \rho_{g}\left(1+\frac{r}{\mathrm{r}_{\mathrm{e}}}-\frac{A_{P} r^{3}}{\mathrm{G} M}\right)$.

Note that the gravitational theory in this paper does not necessarily deny existing physics. The complementarity principle is that a complete description can be made by considering that mutually contradictory elements are realized simultaneously or are the same. For this reason, from the complementarity principle, a phenomenon can be described from not only one viewpoint but also another viewpoint. Therefore, the gravitational theory in this paper only describes gravity from a different viewpoint from existing physics.

\section{Acknowledgments}

I would like to thank my family for supporting me.

\section{References}

[1] S. A. Fulling, "Nonuniqueness of Canonical Field Quantization in Riemannian SpaceTime," Physical Review D 7 (10), 2850-2862, (1973).

[2] P. C. W. Davies, "Scalar production in Schwarzschild and Rindler metrics," Journal of Physics A 8 (4), 609-616, (1975).

[3] W. G. Unruh, "Notes on black-hole evaporation," Physical Review D 14 (4), 870-892, (1976).

[4] J. D. Bekenstein, "Black holes and entropy," Physical Review D 7, 2333, (1973).

[5] S. W. Hawking, "Particle Creation By Black Holes," Commun Math. Phys. 43, 199-220, (1975). 
[6] E. P. Verlinde, "On the Origin of Gravity and the Laws of Newton," arXiv:1001.0785.

[7] W. G. Unruh, "Black Holes, Dumb Holes, and Entropy," In Callender, C. (ed.). Physics meets Philosophy at the Planck Scale, Cambridge University Press. pp. 152-173, Eq. 7.6, (2001).

[8] "Planck reveals an almost perfect Universe," ESA, 19 May 2021.

[9] S. Phelps, A. Nusser, and V. Desjacques, "The mass of the Milky Way and M31 using the method of least action," arXiv:1306.4013.

[10] P. R. Kafle, S. Sharma, G. F. Lewis, and J. Bland-Hawthorn, "On the Shoulders of Giants: Properties of the Stellar Halo and the Milky Way Mass Distribution," arXiv:1408.1787.

[11] T. Licquia and J. Newman, "Improved Constraints on the Total Stellar Mass, Color, and Luminosity of the Milky Way," American Astronomical Society, AAS Meeting \#221, \#254.11. 221: 254.11 (2013).

[12] P. J. McMillan, "Mass models of the Milky Way," arXiv:1102.4340.

[13] S. Gillessen, P. Plewa, F. Eisenhauer, R. Sari, I. Waisberg, M. Habibi, O. Pfuhl, E. George, J. Dexter, S. V. Fellenberg, T. Ott, and R. Genzel, "An Update on Monitoring Stellar Orbits in the Galactic Center," arXiv:1611.09144.

[14] X. Dai, S. Steele, E. Guerras, C. W. Morgan, and B. Chen, "Constraining Quasar Relativistic Reflection Regions and Spins with Microlensing," arXiv:1901.06007.

[15] H. Dehmelt, "A Single Atomic Particle Forever Floating at Rest in Free Space: New Value for Electron Radius," Physica Scripta. T22: 102-110, (1988).

[16] "Lambda-CDM model," Wikipedia, 19 May 2021.

[17] H. E. Bond, E. P. Nelan, D. A. VandenBerg, G. H. Schaefer, and D. Harmer, "HD 140283: A Star in the Solar Neighborhood that Formed Shortly After the Big Bang," arXiv:1302.3180.

[18] "ON the EXPANSION of the UNIVERSE," NASA Glenn Research Center, 19 May 2021.

[19] T. Ishii, "Ippan sotaisei riron wo ippo ippo sushiki de rikai suru (Understanding the general theory of relativity step by step with mathematical formulas)," Tokyo: Beret Shuppan, 655-657, (2019). 
[20] S. Koide, "Butsurigaku santeiban (Physics third edition)," Tokyo: Shokabo, 34-37, (2000).

[21] B. Mashhoon, F. Gronwald, and H. I. M. Lichtenegger, "Gravitomagnetism and the Clock Effect," arXiv:gr-qc/9912027.

[22] S. J. Clark and R. W. Tucker, "Gauge Symmetry and Gravito-Electromagnetism," arXiv:gr-qc/0003115. 